\title{
Plant-soil interactions of range-expanding plants
}

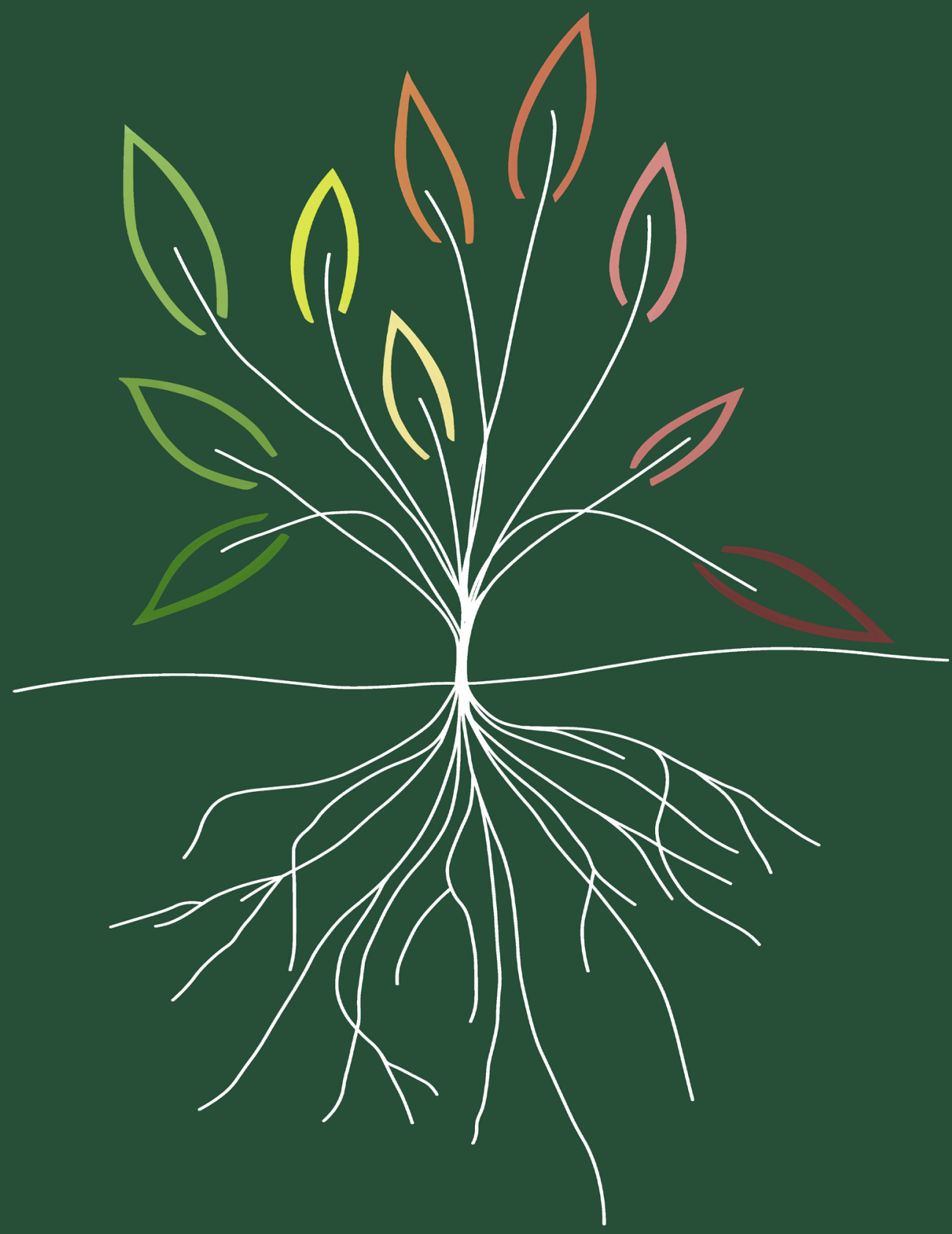

Marta Manrubia Freixa 



\title{
Plant-soil interactions of range-expanding plants
}

\author{
Marta Manrubia Freixa
}




\section{Thesis committee}

\section{Promotor}

Prof. Dr W. H. van der Putten

Special Professor Functional Biodiversity, Wageningen University \& Research Netherlands Institute of Ecology, Wageningen

\section{Co-promotor}

Dr G. F. Veen

Junior Group Leader

Netherlands Institute of Ecology, Wageningen

\section{Other members}

Prof. Dr G. B. De Deyn, Wageningen University \& Research

Dr M. te Beest, Utrecht University

Prof. Dr J. H. C. Cornelissen, VU Amsterdam

Dr E. J. Sayer, Lancaster University, UK

This research was conducted under the auspices of the C.T. de Wit Graduate School for Production Ecology \& Resource Conservation (PE\&RC) 


\title{
Plant-soil interactions of range-expanding plants
}

\author{
Marta Manrubia Freixa
}

Thesis

submitted in fulfilment of the requirements for the degree of doctor at Wageningen University

by the authority of the Rector Magnificus,

Prof. Dr A.P.J. Mol,

in the presence of the

Thesis Committee appointed by the Academic Board

to be defended in public

on Friday 7 December 2018

at 11 a.m. in the Aula. 
Marta Manrubia Freixa

Plant-soil interactions of range-expanding plants 184 pages.

PhD thesis, Wageningen University, Wageningen, The Netherlands (2018) With references, with summaries in English, Catalan and Dutch ISBN: 978-94-6343-531-4

DOI: https://doi.org/10.18174/462576 
"Keep Ithaka always in your mind. Arriving there is what you're destined for.

But don't hurry the journey at all.

Better if it lasts for years,

so you're old by the time you reach the island, wealthy with all you've gained on the way, not expecting Ithaka to make you rich"

Fragment of "Ithaka" by K.P. Kavafis Translated by E. Keeley 



\section{Table of contents}

Chapter 1 General introduction

Chapter 2 Nutrient content in plants and soils of range-expanding plant species in their original and expansion ranges compared to natives

Chapter 3 Belowground consequences of intracontinental range-expanding plants and related natives in novel environments

Chapter 4 Rhizosphere and litter feedbacks to range-expanding plant species and related natives

Chapter 5 Soil functional responses to drought under range-expanding and native plant communities

Chapter 6 General discussion

Sumari

Samenvatting

Acknowledgements

About the author

List of publications

Graduate school training and courses 


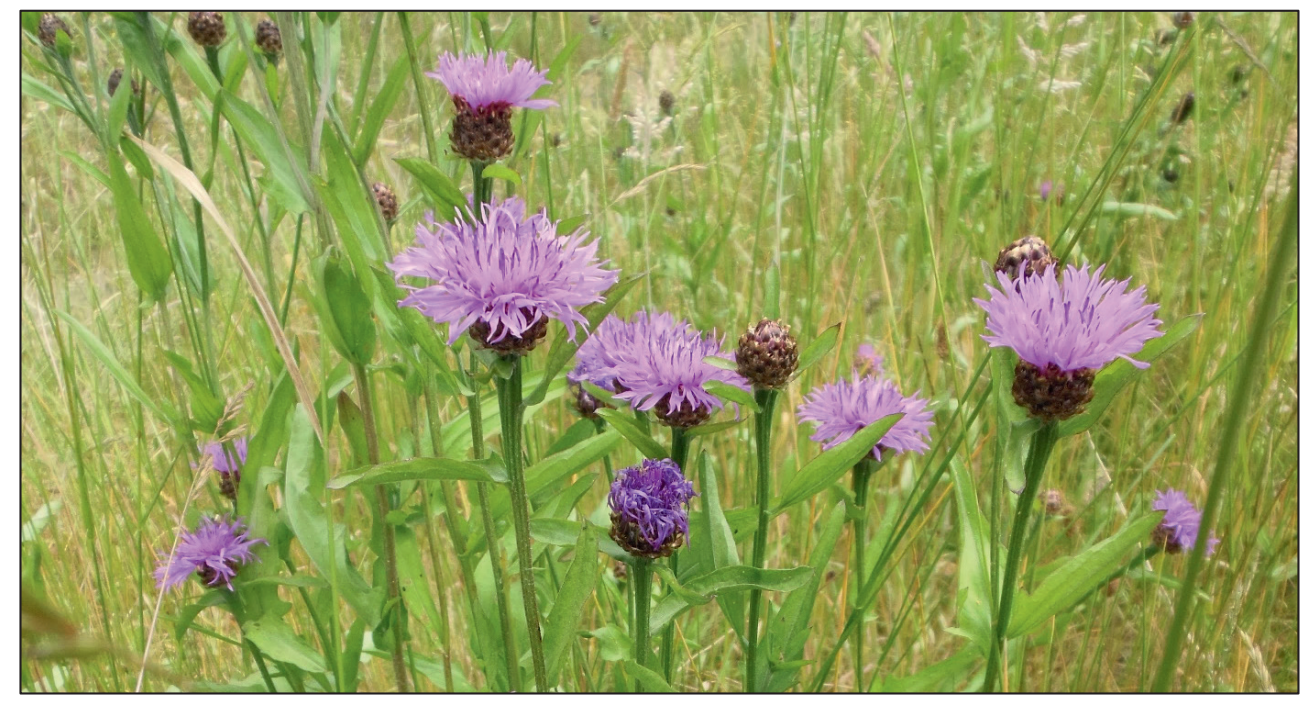


Chapter 1

General introduction 
Anthropogenic activities during the last century have had a profound impact on greenhouse gas balances at a global scale. One of the clearest consequences of the rapid increase of greenhouse gas emissions is the rise of the mean global temperature (IPCC 2014). Quite some plant species are responding to a warming climate by expanding their ranges to higher latitude and altitude regions within continents (Chen et al. 2011). Similarly to introduced exotic species that become invasive, the arrival of range-expanding plant species in recipient ecosystems may have consequences for the composition and functioning of ecological communities, both of native plant species and soil communities (Morriën et al. 2010). In this thesis, I study plant species that expand their range as a response to current global warming and I focus on how these species interact with the abiotic soil environment, the soil microbial communities, and how they influence soil community functioning compared to native plant species. I will especially focus on the impacts of range-expanding plant species on litter decomposition and soil nutrient cycling, which regulate ecosystem carbon balance and the provisioning of nutrients for plant growth.

\section{Climate change and plant responses}

Global biochemical cycles have been profoundly modified by human activities (Vitousek et al. 1997), with important consequences for the climate system. The concentration of carbon dioxide in the atmosphere has now increased by $40 \%$ with respect to pre-industrial times. The increase in greenhouse gas emissions, mainly derived from fossil fuel emissions, has triggered an increase on global average surface temperatures of $0.6^{\circ} \mathrm{C}$ over the $20^{\text {th }}$ century (IPCC 2014). This temperature increase varies locally and has especially been prominent in areas of high latitudes (Fig. 1.1). All projected climate scenarios predict that global temperature will continue to rise during the $21^{\text {st }}$ century, which may further increase carbon dioxide emission from natural ecosystems (Crowther et al. 2016).

Plant species can respond to climate warming in different ways. Plants can undergo genetic and phenotypical adaptation induced by climate warming. Adaptation can result, for example, in phenological changes such as earlier flowering time, or longer growing season (Peñuelas and Filella 2001, Cleland et al. 2007). However, adaptation processes in the future may be critically limited by the rapid rates at which the climate changes and by decreased gene flow between fragmented habitats (Davis and Shaw 2001, Jump and Penuelas 2005). Thereby, climate warming can also lead to local extinctions of plant species 
that are unable to adapt or disperse (Thomas et al. 2004). Wild plant species can also respond to a warming climate by expanding their ranges to higher latitude and altitude regions within continents (Parmesan and Yohe 2003, Lenoir et al. 2008, Chen et al. 2011). It has been estimated that the average distribution of species has shifted polewards at a rate of $16.9 \mathrm{~km}$ per decade (Chen et al. 2011). Due to the increase of temperature in areas of high latitude, especially in the northern Hemisphere (Fig. 1.1), habitats have become more suitable for thermophilic plant species. For example, in the Netherlands, the number of plant species from warmer climatic regions has increased substantially during the last decades (Tamis et al. 2005, NDFF 2018). Even though the introduction of some of these species may have been induced by human activity, natural range expansion and the spread of plant species is likely to be enabled by warming climate.

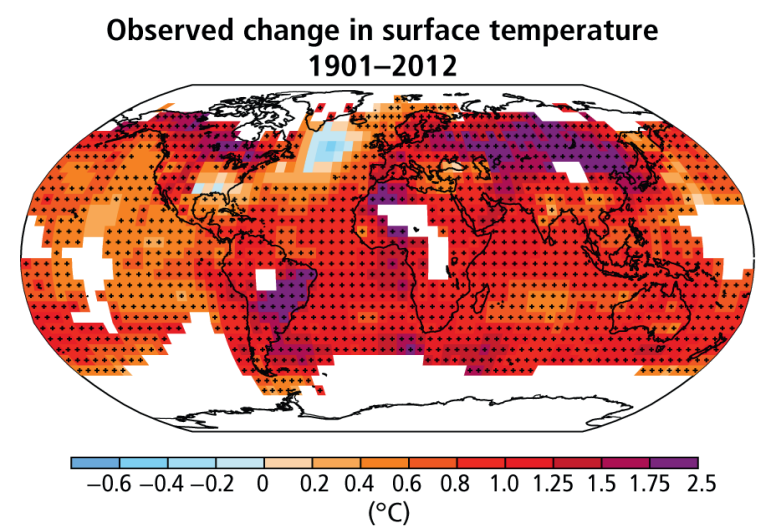

Fig. 1.1. Map of the observed surface temperature change, from 1901 to 2012 (source: IPCC, 2014). Trends have been calculated where data availability permitted a robust estimate (i.e., only for grid boxes with greater than $70 \%$ complete records and more than $20 \%$ data availability in the first and last $10 \%$ of the time period), other areas are white. Grid boxes where the trend is significant, at the $10 \%$ level, are indicated by a sign.

Plant species that expand their range within continents establish in ecosystems outside their native range. Similarly, plant species that have been introduced to other continents also establish outside their native range. In the ecological literature, the latter are traditionally referred to as (introduced) exotic species (Keane and Crawley 2002). While it can be argued that plants that expand their range within a continent into previously uncolonized higher latitude areas are exotic species as well, I will refer to them as "range- 
expanders" in this thesis. In contrast, I will use the term exotic species to refer to studies that have investigated the causes and consequences of intercontinental plant introductions. Numerous studies have examined successfully established exotic plants that become invasive and induce important changes on the composition and functioning of the invaded ecosystems (Ehrenfeld 2010, Vilà et al. 2011, Pyšek et al. 2012). However, little is known about the manner that range-expanding plant species may affect the composition and functioning of ecosystems in the new range.

\section{Plant-soil interactions}

All plant species, including introduced exotic plants, can induce changes in the soil biotic and abiotic components that affect their own performance and the performance of subsequent plant generations (Wardle et al. 2004, Bezemer et al. 2006). This phenomenon is known as plant-soil feedback (Bever et al. 1997). Individual plants and soil communities interact with each other in a direct or indirect manner, and the outcome of these interactions can have positive, negative or neutral feedback effects to plants. According to the general framework proposed by Wardle et al. (2004), soil communities can affect plants in a direct manner when soil organisms associate with living plant roots or in an indirect manner when soil organisms that consume dead plant material provide the nutrients needed by plants to grow (Fig. 1.2). Mechanisms of direct plant-soil interactions include pathogenesis, herbivory or symbiotic-mutualistic relationships. The association of pathogens, herbivores and symbiotic mutualists with a host plant has immediate effects to its performance. In contrast, the soil decomposer subsystem transforms organic plant material into inorganic plant-available nutrients via litter decomposition, thereby affecting plant performance indirectly. Besides the strong influence of climatic conditions on decomposition processes, plant traits and soil decomposer communities have been shown to regulate decomposition rates in soil (Cornelissen et al. 2004, Cornwell et al. 2008, Bradford et al. 2017). These indirect interactions mediated by plant litter and the functioning of decomposer communities are essential for understanding ecosystem productivity (van der Heijden et al. 2008) and the soil carbon balance (De Deyn et al. 2008, Sayer et al. 2011). 


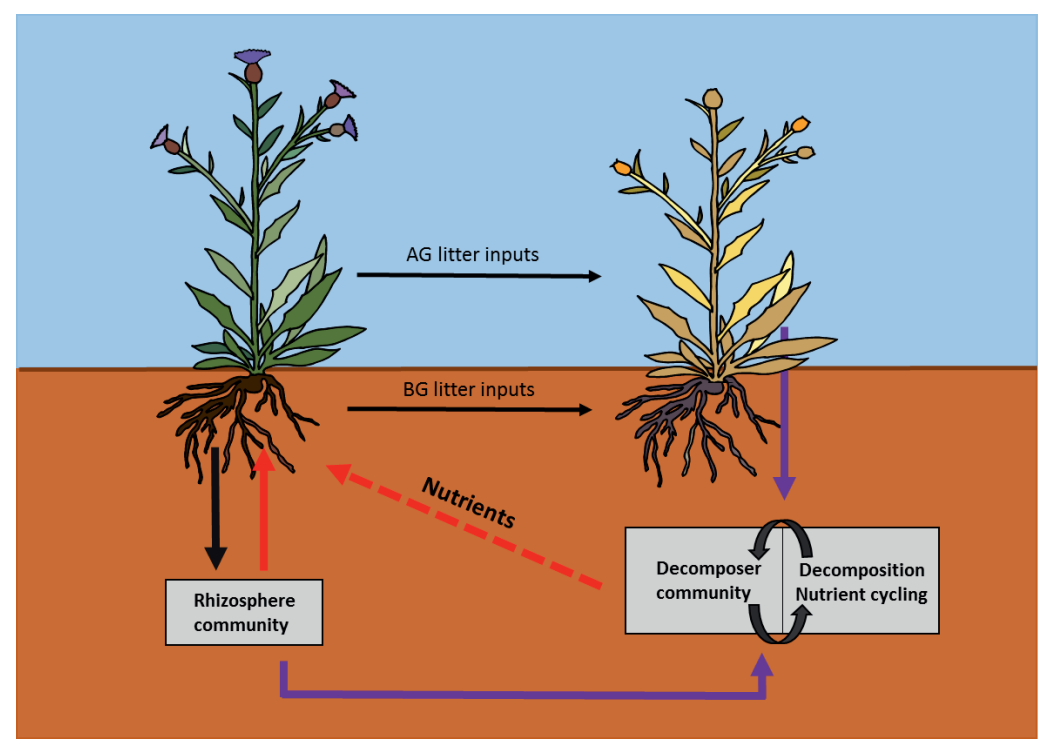

Fig. 1.2. Conceptual schematic of plant-soil interactions mediated by the rhizosphere and the plant litter pathways (after Wardle et al. 2004). Plants influence rhizosphere communities as they grow (black arrow), which can affect plant growth via direct effects (solid red arrow). When rhizosphere communities are dominated by enemies, direct effects will be negative for plant performance. When rhizosphere communities are dominated by mutualistic symbionts, direct effects will be positive for plant performance. After plants senesce, death aboveground $(A G)$ and belowground $(B G)$ plant material is left in the soil. Litter quantity and quality influences the soil decomposer community and decomposition activity (purple arrow, top). Through decomposition activity, decomposer communities mineralize and mobilize nutrients in plant litter and, thus, affect plant performance indirectly (dashed red arrow). The rhizosphere community may also influence the decomposer community and decomposition activity (solid purple arrow, bottom) and, thereby, affecting plant performance indirectly (dashed red arrow). Note that among decomposer microorganisms there can be facultative saprophytic pathogens, which can directly affect plant performance as litter decomposes.

Unraveling mechanisms underlying plant-soil feedbacks is important to understand plant community dynamics, including dynamics of exotic plant species, during invasions (Klironomos 2002, van der Putten et al. 2010, Dostál et al. 2013, Dostálek et al. 2016). In this way, research efforts trying to elucidate the underlying mechanisms of invasion success have focused on understanding the interactions between exotic plants and native plant and soil communities. It has been suggested that range-expanding plant species may undergo shifts in the composition and structure of their associated above and belowground communities as they expand their range (van der Putten 2012). A main reason for this asynchronous range expansion can be that plants and their associated organisms have different requirements and rates of dispersal (Berg et al. 2010). 
Consequently, and similarly to intercontinental invasive species, range-expanding plants may then be subjected to different plant-soil interactions in the new range as compared to the original range which can have consequences on their establishment and performance (Van Grunsven et al. 2010).

\section{Plant-soil interactions in exotic plant species}

Biotic interactions between exotic plants and soil organisms in the invaded ecosystem can facilitate or control the spread of the exotic plant species (Keane and Crawley 2002). Exotic species can experience enemy release when their specialist enemies are not present in the invaded range and they are not recognized by the enemies present in the invaded range (Mitchell and Power 2003, Verhoeven et al. 2009). As a result of this evolutionary mismatch, exotic plant species can have an advantage over native species in the new range when native species experience more negative effects of above and belowground enemies (Andonian et al. 2011). Furthermore, exotic species that become invasive have been shown to gain competitive advantage by the release of phytochemical compounds that are novel to the invaded community and can have allelopathic, defensive or antimicrobial properties (Callaway and Ridenour 2004, Cappuccino and Arnason 2006). On the other hand, the native plant and soil communities in the invaded range can also exert biotic resistance to the exotic species, thereby reducing their performance (Levine et al. 2004). However, because most published studies to date focus on exotic species that have become invasive in their new range, relatively little is known about mechanisms of biotic resistance.

Exotic plant species can also affect the soil community by providing litter and root exudates that differ from those of native plants. As plants grow, they release root exudates to the rhizosphere soil surrounding their roots. Root exudates are composed of numerous chemical compounds with a wide array of functions (Walker et al. 2003, Philippot et al. 2013). For example, root exudates can act as signaling molecules that can interfere in direct plant-soil interactions because they may attract or repel plant-specific pathogens or symbionts (Paterson et al. 2007, Badri and Vivanco 2009). However, root exudates can also play a role in indirect plant-soil interactions by stimulating litter and organic matter decomposition in the soil (Cheng et al. 2003, Kuzyakov et al. 2006, de Graaff et al. 2010). Thus, root exudates contribute to mobilize mineral nutrients that can be acquired by plants again. Because root exudation seems to be plant species-specific (Badri and Vivanco 2009, Philippot et al. 2013), root exudation may be a pathway by which exotic plant species affect 
soils differently than natives (Bradford et al. 2012). Differences in litter chemistry and litter quantity between exotic and native plant species can also affect soil decomposer communities and decomposition rates in the invaded ecosystems. The degree of recalcitrance (e.g. lignin or dry matter content) and the presence of secondary metabolites in the litter are strong drivers of decomposition rates (Cornwell et al. 2008, Freschet et al. 2012, Chomel et al. 2014). Non-native plant species, both in the case of exotics and rangeexpanders, have been shown to have more unique and novel metabolites in their tissues according to a number of studies (Cappuccino and Arnason 2006, Macel et al. 2014). Because of these differences, they may affect decomposition and decomposer communities in the soil differently. Such effects may remain in soil as legacy effects that can influence plant-soil feedbacks (Ehrenfeld 2003, Meisner et al. 2012).

The mechanisms of plant-soil interactions that enable exotic species to perform well in other continents might be the same that also influence range-expanding plant species that establish in new ranges within continents. For example, range-expanding plant species have been shown to perform better in soils of the new range than in soils of the original range, which may indicate enemy release during range expansion (Van Grunsven et al. 2010, Van Nuland et al. 2017). Some studies have also shown that, within the new range, range-expanding plant species experience less negative pressure from soil communities than related native species (van Grunsven et al. 2007, Engelkes et al. 2008, Morriën et al. 2012, Morriën et al. 2013). These results suggest that the establishment and performance of range-expanding plant species in the new range may be promoted by low enemy pressure. Yet, we have a poor understanding of how range-expanding plant species interact with decomposer communities in the soil. Nevertheless, indirect interactions via decomposition play an important role in nutrient provisioning for plant growth and drive crucial ecosystem functions such as litter decomposition and nutrient cycling. Therefore, in this thesis I examine the functional consequences of range-expanding plant species for the soil communities in the new range via indirect plant-soil interactions.

\section{Functional consequences of exotic plant invasions}

\section{Soil carbon and nutrient cycling}

Exotic plants have been shown to alter soil nutrient dynamics (Ehrenfeld 2003). In global meta-analyses, exotic plant species appeared to enhance ecosystem primary productivity, 
carbon and nitrogen pools and fluxes in the soils of invaded ecosystems relative to native ecosystems (Liao et al. 2008, Vilà et al. 2011). Empirical studies have found that effects of exotic plants can be detected at the level of decomposition rates and enzyme activities in the soil (Kourtev et al. 2002b, Allison and Vitousek 2004, Allison et al. 2006). Despite these general trends, the effects of exotic plant species on soil processes are not unidirectional and can be highly variable. One reason for this may be that the impacts of exotic species depend on the characteristic of the site and the plant species compared (Ehrenfeld 2010). In that case, local factors of individual invaded sites (such as the identity and the traits of the resident plant community, the soil biotic resistance or abiotic factors), may contribute to determining the community and ecosystem responses to the introduction of the exotic species.

\section{Feedback effects of plant litter}

Research on plant-soil feedbacks under controlled greenhouse conditions has largely focused on legacy effects driven by interactions between 'living' plant roots and soil communities, whereas less is known about legacy effects driven by litter inputs to the soil (Ehrenfeld et al. 2005, Kulmatiski et al. 2008). However, it has been shown that plant-soil feedback effects mediated by plant litter can increase the performance of exotic plant species over the performance of resident native species (Eppinga et al. 2011, Elgersma et al. 2012, Mariotte et al. 2017). In order to have positive effects of litter feedbacks to invasion, the litter of the exotic plant species has to alter soil nutrient status and, at the same time, the exotic plant species must benefit from this change in soil nutrients more than the native species. It is often the case that plant litter from exotic species enhances decomposition and, therefore, leads to higher nutrient availability (Ehrenfeld 2003, Allison and Vitousek 2004). Besides, exotic plant species seem to grow in soils that have higher nutrient content than soil of natives (Sardans et al. 2017) and to be faster and more efficient than native plant species during nutrient acquisition (Blumenthal et al. 2009, Liu and van Kleunen 2017). Thereby, in fertilized or nutrient rich soils exotic species are more likely to become dominant. Altogether, this suggests that exotic plant species may develop positive feedback through their litter that enhances their dominance. In the case of rangeexpansion, it has been shown that range-expanding plants may not exploit soil nutrients better than natives but that they have more nutrient-rich shoot tissues than native species (Meisner et al. 2011). Nutrient-rich litter consequently increased decomposition rates, but did not inhibit the germination of native plant species (Meisner et al. 2012). However, the 
consequences of plant litter feedbacks on the growth of range-expanding plants beyond the seedling stage and in combination with rhizosphere feedbacks remain unclear.

\section{The role of specialized soil communities}

As plants establish outside their native range, specific interactions between plants and their co-evolved above and belowground communities may be disrupted due to the lack of these specialized organisms in the new range (van der Putten 2012). Thereby, novel interactions with soil organisms may be established in the new range. While empirical evidence suggests that both exotic and range-expanding plant species may benefit from escaping soil borne enemies or from beneficial soil organisms in the new range (Keane and Crawley 2002, Mitchell and Power 2003, Van Grunsven et al. 2010), less is known about the role of specialized decomposers. In theory, plants may also lose positive interactions with specialized decomposer communities, as the "home-field advantage" hypothesis predicts that litter decomposition is accelerated beneath the plant it originates from ("home") than beneath another plant ("away") due to the presence of specialized decomposer communities (Gholz et al. 2000, Ayres et al. 2009a). Although home-field advantage effects are highly context-dependent and variable across studies (Veen et al. 2015b), decomposer communities in the soil have been shown to adapt to the history of specific litter inputs (Keiser et al. 2011). It remains unknown whether specialized decomposer communities may be lost during range expansion and whether rangeexpanding plants may develop specialized communities in their new range over time. Yet, interactions with specialized decomposer communities may be important for local plant performance as decomposition drives soil nutrient availability locally. Thereby, addressing the role of specialist decomposer communities in the context of plant range expansion may help to predict the consequences of range-expanding plant species for ecosystem functioning in the new range.

\section{Interactions of extreme weather events and plant range-expansion}

Soil microbial communities and soil ecosystem functioning might not only be affected by range-expanding plants, but are also subjected to direct impacts of climate change. The consequences of individual factors of climate change such elevated $\mathrm{CO}_{2}$, warming and drought events on plants and soils have been widely studied (Rustad et al. 2001, de Graaf et al. 2006, Fuchslueger et al. 2014). Climate directly influences soil microbial communities 
and their activity (Waldrop and Firestone 2006a). The physiological traits of the microbial communities and their interactions with other soil biota will determine to what extent microbial activity is affected by climate change (Allison and Martiny 2008, Griffiths and Philippot 2013, Crowther et al. 2015). Soil microbes and their activity can also be affected indirectly through changes in plant community composition and plant inputs (Kardol et al. 2010, Sayer et al. 2017). Therefore, in order to understand how ecosystems may respond to predicted future climate scenarios, it is important to integrate both plant community changes and direct climate effects (Bardgett and van der Putten 2014, Classen et al. 2015). The arrival of range-expanding plant species to recipient ecosystems at higher latitudes and altitudes will modify plant community composition and, thereby, have an indirect impact on soil microbial communities and processes by the provisioning of resources. Furthermore, projected climate scenarios in the Netherlands predict that drought during summer periods will become more recurrent (KNMI 2015). Therefore, the presence of range-expanding plant species from typically warmer and drier areas is likely to interact with extreme climate events. However, little is known about the effect of such interactions on important soil functions, such as litter decomposition and nutrient cycling.

\section{Objectives of this thesis}

In this thesis, my aim is to investigate plant-soil interactions of range-expanding plant species that operate through plant inputs to the soil and to determine how these interactions compare to those of native plant species in the new range. Therefore, in my thesis, I focus mostly on indirect plant-soil interactions, which are mediated by the decomposer subsystem. In this thesis, I investigate how range-expanding plant species affect soil communities and functions such as litter decomposition and nutrient cycling. I aim to determine the role of specialized decomposer communities in range-expanding plants and that of plant-soil feedback effects mediated by litter inputs. I also study how plant range-expansion interacts with extreme climatic events to modify soil functions.

\section{Study system and experimental approach}

Plant range expansions within continents provide a unique study system to investigate how non-native plant species interact with above and belowground communities. A main difference with studies about exotic species introduced to other continents is that gradients 
of range expansion allow the test hypotheses both in the new and native range, because it is relatively easy to trace back where these plants originated from and also because there are less logistic constraints to do so (Van Grunsven et al. 2010). In Europe, plants that are native from the Mediterranean regions expand their range northwards, resulting in the arrival of thermophilic plants to the Netherlands, among other regions (Tamis et al. 2005). The design of a sampling scheme along this latitudinal gradient of range expansion enables to study how communities around range-expanding plants shift along the latitudinal gradient and how species perform in both the native and new ranges.

Throughout this thesis, I pay special attention to the role of plant origin (i.e. rangeexpander or native) in indirect interactions. The role of plant origin in the effects of plant species in ecosystems has not been explicitly addressed before as, generally, studies have been carried out to compare exotic plant species with resident natives that may be more or less similar to the exotic species besides their difference in origin. Thereby, in those studies several other factors, such as trait differences between compared species, may determine the impact of the exotic species, whereas the underlying effect of plant origin (native vs non-native) is not known. For this reason, in the experimental approach throughout my thesis I used phylogenetically controlled comparisons which allow to test the effect of origin in species that are otherwise ecologically similar (Agrawal et al. 2005).

\section{Thesis outline}

In Chapter 2, I analysed plant chemical composition and soil nutrient availability from field populations of range-expanders and native plant species both in the expansion and the original range. I tested the hypothesis that range-expanding plant species have higher nutrient concentrations, both in their leaf tissues and in their associated soils, than native species in the new range. These patterns are characteristic in exotic plant species that are invasive in other continents (Pyšek et al. 2012, Sardans et al. 2017), and thereby, may be an indicator of plant invasiveness among plant species that expand their range within continents.

In Chapter 3, I studied the bacterial communities that assemble in the rhizosphere of range-expanding plants and compared them to those of related native plant species. In this experiment, I aimed to test the effect of plant origin on bacterial community 
composition and soil functioning by teasing apart the role of plant traits and the role of site-specific differences on soil communities from the role of plant origin.

In Chapter 4, I investigated plant-soil feedback effects through rhizosphere and plant litter legacies of range-expanding plant species and related natives. Here, I incorporated the plant litter pathway to plant-soil feedbacks to determine how litter feedbacks contribute to the performance of the range-expanding and native plant species in the new range. In the feedback phase, I focused both on plant growth responses and on decomposition responses of subsequent plant litter to conditioned soils.

In the last experimental chapter, Chapter 5, I was interested in how plant communities of range-expanders sustained soil functioning in terms of litter decomposition and how these functions were affected in comparison to native communities under extreme drought events. Apart from the origin of the plant community, the experiment also included soil biota originating from the original range of the range-expanding plant species. Decomposition, basal soil respiration and enzyme activity were measured at different times after an artificial drought treatment, comparable to the more frequently occurring summer droughts, to assess performance of soil communities.

In Chapter 6, I provide a general discussion and synthesis of the main findings of this thesis. I compare these results to those of the literature on both range expansion and exotic invasive species. Finally, I present ideas for further research. 


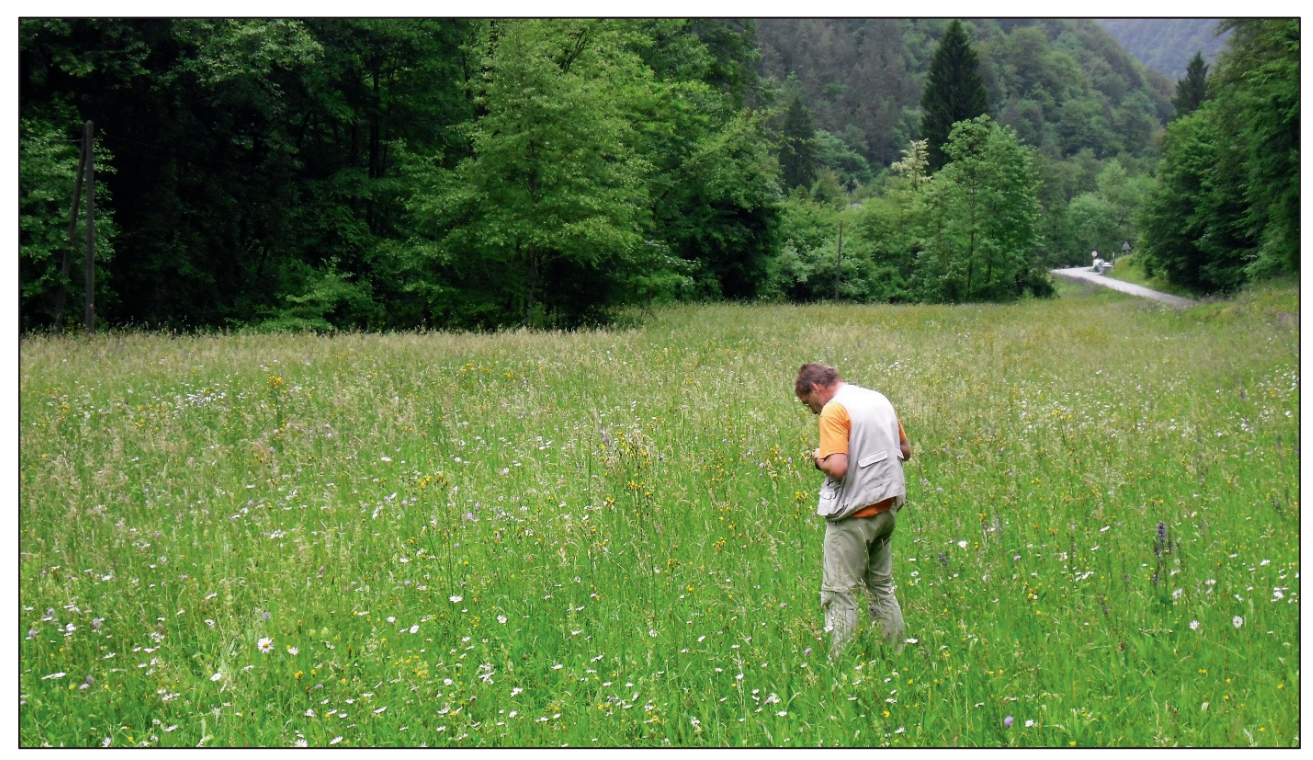




\section{Chapter 2}

\section{Nutrient content in plants and soils of range- expanding plant species in their original and expansion ranges compared to natives}

Marta Manrubia, Ciska Veen, Olga Kostenko, Stefan Geisen, Freddy ten Hooven, Carolin Weser, L. Basten Snoek, Janneke Bloem, Danka Caković, Tatjana Čelik, Kadri Koorem, Nikos Krigas, Kelly S. Ramirez, Maria Tsiafouli, Branko Vreš, Rutger A. Wilschut \& Wim H. van der Putten 


\begin{abstract}
Introduced exotic plant species that become invasive often have higher nutrient concentrations in their tissues and occur in soil patches with higher nutrient availability than co-occurring native species. Opposite to the many studies on nutrient contents of exotic plant species that have been introduced from other continents, no such studies are known on plant species that expand their range naturally within continents under current climate warming. Here, we investigated whether range-expanding plant species may also have higher nutrient concentrations in their leaves and associated soils than native species. We collected leaf and soil samples from European field populations of range-expanding plants in the new (north) and original (south) ranges (from the Netherlands to Montenegro). In order to avoid confounding effects of plant relatedness in the comparison between range expanders and co-occurring native plant species, we compared range expanders with congeners that are native in both the original and invaded habitats. We also examined range-expanding plant species that do not have related native species along the expansion range. Our results show that plant and soil chemistry varied between the north and south ranges, but the direction and size of these latitudinal differences was dependent on the parameter and the plant species considered. Differences in plant and soil nutrients of range-expanding and related native plant species were linked to species identity and there was no general pattern associated with plant species origin. We conclude that there are species-specific differences between plants that expand their range and their related natives, but that in general range-expanding plant species do not have higher nutrient contents in plant tissues and associated soil in their novel range.
\end{abstract}




\section{Introduction}

Predicting plant invasion success is a main challenge in invasion ecology. Previous work has aimed at elucidating prevalent traits of invasive plant species that may drive their invasiveness and characteristics of the recipient environments that may drive their susceptibility to invasions (Rejmanek and Richardson 1996, Hayes and Barry 2008, Ordonez et al. 2010, te Beest et al. 2012). Identifying such traits and characteristics may help to predict when and where exotic plant species may impact native ecosystems (Levine et al. 2003). Invasion by exotic plants has often been linked to the availability of nutrients in the soil and their turnover rates (Ehrenfeld 2010). Soil nutrient concentrations are generally higher in soils occupied by exotic invasive plants than in soils of natives (Pyšek et al. 2012, Sardans and Peñuelas 2012). A meta-analysis indicated that this pattern is particularly strong in nutrient-poor ecosystems, where exotic invasive plants have higher nutrient levels in their soils and leaves than adjacent natives (Sardans et al. 2017). Even though this analysis does not reveal whether invasive species cause changes in nutrient availability or are affected by it, empirical studies widely suggest that exotic invasive species can alter available soil nutrient pools and mineralization (Ehrenfeld 2003, Kourtev et al. 2003, Allison and Vitousek 2004, Ashton et al. 2005, Gómez-Aparicio and Canham 2008, Liao et al. 2008, Vilà et al. 2011, Castro-Díez and Alonso 2017).

The relationship between exotic plant species and high soil nutrient availability may be either a cause or a consequence of invasion. The growth of exotic plant species has been shown to benefit more than natives from fertilization pulses and overall high amount of nutrients available in the soil (Valliere and Allen 2016, Liu and van Kleunen 2017). Thus, high availability of soil nutrients may facilitate the establishment of exotic plant species. On the other hand, plant litter of exotic plant species has been shown to decompose more readily than that of surrounding natives because it contains higher nutrient concentrations, and thus has overall higher quality (Allison and Vitousek 2004, Ashton et al. 2005). Exotics plant species may, therefore, increase soil nutrient availability through their litter inputs to the soil (Wardle and Peltzer 2017), which can create a positive feedback to their own growth. In general, high nutrient availability in the soil, both via pre-existing soil condition or via litter effects, may become even more important when exotic plant species are released from their specialized enemies as shown by Blumenthal et al. (2009). 
Currently, a novel type of exotic plant introduction can take place intra-continentally. Enabled by climate warming, some plant species are able to expand their geographic range to areas of higher latitudes and altitudes within continents (Parmesan and Yohe 2003, Chen et al. 2011). Thereby, processes of range-expansion also result in the introduction of non-native species in those ecosystems, which may or may not include invasive plant species (Morriën et al. 2010). Such range-expanding plants may lack soil-borne enemies in the new range, which can enhance plant performance (Van Grunsven et al. 2010), and favour plant success in the new range. In contrast to introduced exotic invaders, less is known about the interactions between range-expanding plants and the abiotic soil environment. Yet, examining the relationship between plant and soil nutrient content in plant range expansions may help to identify general trends characteristic of invasive exotic species. While in studies on introduced exotic species, exotic plants are often compared with adjacent native vegetation, intracontinental range-expansions enable to compare range-expanding species with related species that are native along the expansion gradient. However, at the same time, many range-expanding plant species within Europe are unrelated to native vegetation (NDFF 2018). The ecology of range expanders that do not have closely related native species in the expansion range may resemble that of exotic invasive species to a larger extent (Strauss et al. 2006, Koorem et al. 2018). Yet, we have a poor understanding of whether range-expanding plants, like introduced exotics, are associated with higher nutrient levels in plant tissues and associated soil, and whether there are differences between range-expanding plant species that do and do not have related native species along the expansion range.

Here, we investigated nutrient concentration in plant leaves and soils of range-expanding plant species, both in the original and the new range. We hypothesized that, in line with introduced exotic invasive plants, 1) range-expanders have higher nutrient concentrations in their leaf tissue than congeneric native plant species in the new range; 2) rangeexpanders have higher nutrient concentrations in their associated soils than congeneric native plant species in the new range. In addition, we examined range-expanders that do not have closely related natives in the expansion range and compared new and original range and their similarity to co-occurring, but unrelated native plant species. Furthermore, we also expected differences in plant chemistry to be consistent in the original and new range, as that would indicate that these are inherent characteristics of the successfully range-expanding plant species. We tested the hypotheses using a dataset of plant and soil chemistry of both range-expanding plant species in their original (hereafter, south) and 
expansion (hereafter, north) ranges in Europe, and native plant species that are native in both ranges.

\section{Materials \& Methods}

Plant and soil sample collection

In the spring and summer of 2014, we collected plant material and soil under all plants along a latitudinal transect in Europe, spanning from Montenegro to the Netherlands. We considered samples collected from mid-Germany and Netherlands to be representative of the North range, and samples collected from Montenegro and Slovenia to be representative of the South range. We selected native plant species from South-East Europe that have expanded their range to North-West Europe within the last 50 years approximately, and that are present in riverine ecosystems of central Netherlands (Tamis et al. 2005). Seven range-expanders were sampled along the latitudinal transect: four of them have a native congener along the latitudinal range (related range-expanders) and three of them do not have native congeners in native flora of the expansion range (unrelated range-expanders). Four native species were harvested to act as control for the related range-expanders: one congeneric native for each range expander. In total, eleven plant species were surveyed. Within each of the sampling locations, we identified three plant populations of each species. Within each population 3 individual plant and soil samples were collected, aiming at 9 individual plant-soil samples per location. We recorded the geographical location of each population, collected middle leaves from plants, which were all sampled when being in the flowering stage, and subsequently took a soil sample from underneath each plant individual. In total, 318 plant and soil samples were collected (Table S2.1). All samples were cooled and brought to the Netherlands Institute of Ecology (Wageningen, the Netherlands) for laboratory analyses. The related range expanders were Centaurea stoebe, Chaerophyllum temulum, Geranium pyrenaicum and Tragopogon dubius. The congeneric natives were Centaurea jacea, Chaerophyllum bulbosum, Geranium molle and Tragopogon pratensis. The unrelated range-expanders selected were Ditrichia graveolens, Lactuca serriola and Rapistrum rugosum.

\section{Soil chemical analyses}

All soils were sieved to $4 \mathrm{~mm}$ and oven dried at $40{ }^{\circ} \mathrm{C}$ for 5 days prior chemical analyses. We extracted soil mineral nitrogen $\left(\mathrm{NO}_{3}{ }^{-}, \mathrm{NO}_{2}{ }^{-}\right.$and $\left.\mathrm{NH}_{4}{ }^{+}\right)$by shaking a $10 \mathrm{~g}$ dry weight equivalent of soil in $50 \mathrm{ml}$ of $1 \mathrm{M} \mathrm{KCl}$ solution for 2 hours. In the extract, we measured soil 
$\mathrm{NO}_{3}-\mathrm{NO}_{2}$ and $\mathrm{NH}_{4}$ concentrations using an auto-analyzer (QuAAtro Segmented Flow Analysis system, SEAL Analytical Ltd., Southampton, UK). A second subsample of dry soil was used to quantify plant-available phosphate (P-Olsen). We extracted phosphate from $2.5 \mathrm{~g}$ of soil in a $0.5 \mathrm{M} \mathrm{NaHCO}_{3}$ solution and measured it using an auto-analyzer (QuAAtro Segmented Flow Analysis system, SEAL Analytical Ltd., Southampton, UK). A third subsample of dry soil was ground using a ball mill. Then, we weighted $5 \mathrm{mg}$ of ground soil into tin cups to prepare for the measurement of elemental concentrations of carbon and nitrogen using and elemental analyser (QuAAtro Segmented Flow Analysis system, SEAL Analytical Ltd., Southampton, UK).

\section{Plant chemical analyses}

Plant material was oven dried at $70^{\circ} \mathrm{C}$ for $48 \mathrm{~h}$, ground and homogenized using a ball mill. We then measured the total amount of elemental $\mathrm{C}$ and $\mathrm{N}$ in $2 \mathrm{mg}$ of ground plant material using an autoanalyser (QuAAtro Segmented Flow Analysis system, SEAL Analytical Ltd., Southampton, UK). The total amount of phosphorous in the plant material was measured in the form of orthophosphate. Orthophosphate was obtained by ignition of ground plant material at $550{ }^{\circ} \mathrm{C}$ and subsequent digestion in an autoclave with $2.5 \%$ potassium persulfate solution. The obtained extract was measured colorimetrically with and autoanalyzer (QuAAtro Segmented Flow Analysis system, SEAL Analytical Ltd., Southampton, UK).

\section{Data analyses}

We performed Principal Component Analyses (PCA) to explore the variation between all samples based on their plant and soil characteristics. We then used redundancy analyses (999 permutations) to test the effect of range and plant origin on explaining the variation among samples. Data were log transformed prior analyses and standardized and centred prior to analyses to enable comparing variables expressed in different units and with different ranges. Multivariate analyses were conducted in CANOCO 5.0 software (Ter Braak and Šmilauer 2012). For simplification, we present separate ordination plots for each plant genus by selecting PCA scores for one genus at a time, but without changing the overall ordination analyses. In this way, figures can be directly compared.

We then used univariate analyses on each of the plant and soil parameters separately. For the plant pairs of related range-expanders and congeneric natives, we used generalized linear models with plant genera, range (North and South) and plant origin (native and 
range-expander) as fixed factors. For the unrelated range-expanders, we used generalized linear models with plant genera and range as fixed factors. Although within one range plants were sampled from three populations, we did not include population as a factor in the model. This is because the distance between individual samples within populations was not controlled for and was highly variable. Prior to univariate analyses, data were logtransformed. We used a post-hoc Tukey test of least square means to determine the effects of plant origin and range within each plant genera using the package "lsmeans" in $\mathrm{R}$ (Lenth 2016). Model residuals plots were inspected for normality. Univariate analyses were performed in R ( $R$ Core Team 2017).

In the data set, two samples had extremely high values for nitrogen availability in the soil (40 and 17 times higher than average). These values were also identified as outliers using the Grubbs test for outliers from the "outliers" package in R (Komsta 2011), and therefore removed from the analyses. This was the case for a Centaurea jacea soil sample collected in the South range and a Lactuca serriola soil sample collected in the South range.

\section{Results}

\section{Principal component analyses}

In a principal component analysis (PCA), we analysed the variation of samples based on the plant and soil chemical parameters measured. The first and second axis of the PCA explained 39 and $20 \%$ of the variation, respectively, in plant chemical composition and soil nutrient availability and chemical composition (Fig. 2.1). Principal Component 1 represented variation in nitrogen and carbon in soil and plants, while Principal Component 2 mainly represented the variation in phosphorous and plant carbon content. Axis 2 mainly separated samples by range (north and south). The variation in axis 1 characterized the variation of samples within the southern part of the range.

Range played a major role in separating the samples (Redundancy Analyses permutation test: pseudo-F $=38.2, p$ 0.001). Plant origin played a smaller role than range, but still significant, in separating the samples in the ordination plot (Redundancy Analysis permutation test: $p s e u d o-F=10.6, p$ 0.001). Differences between samples within the north range were smaller than within the south, indicating that habitat characteristics and plant chemistry were more variable in the south range than in the north. These effects of range and plant origin are apparent when presenting the PCA ordination for each plant genera separately (Fig. S2.1). 


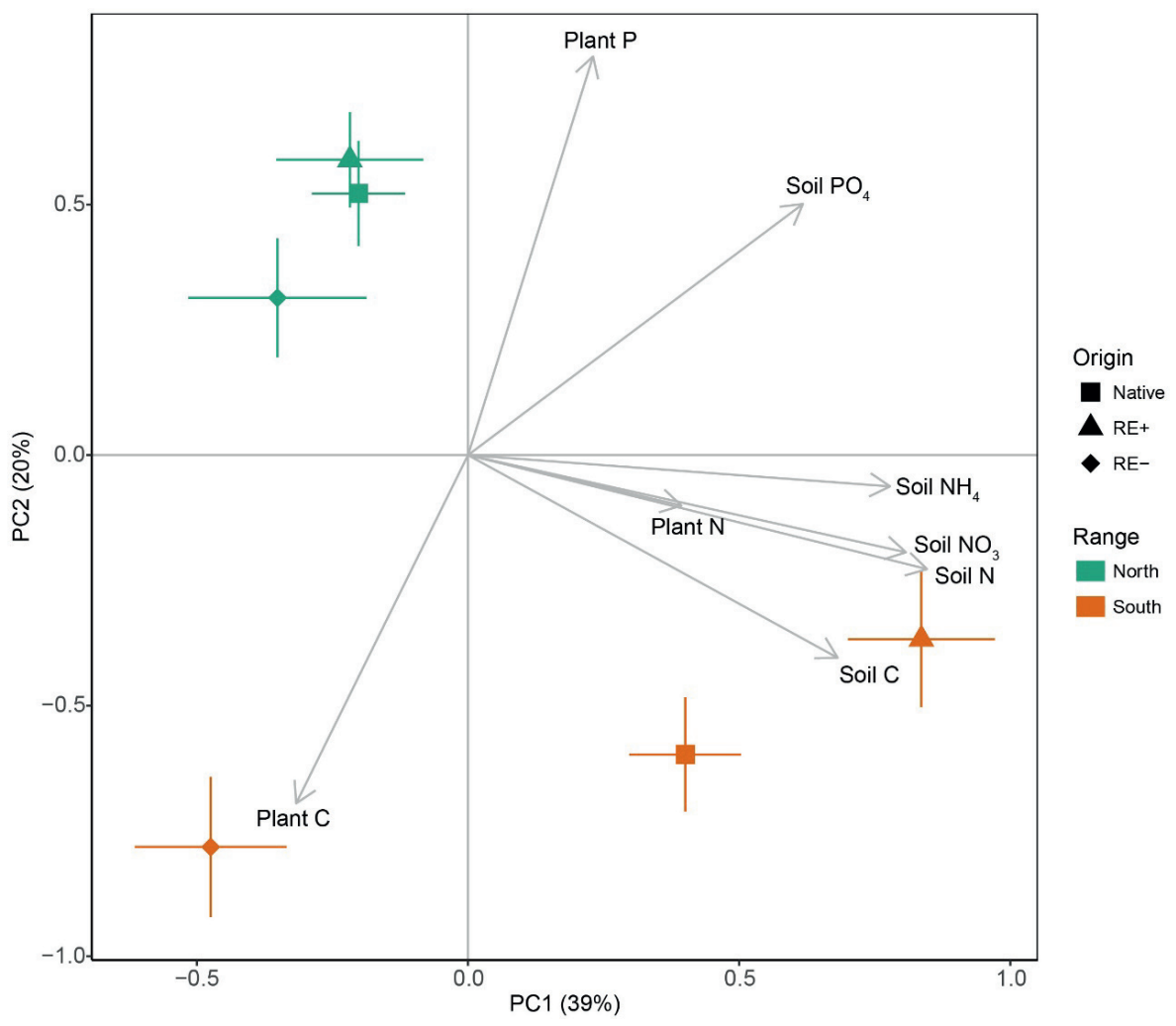

Fig. 2.1. Principal Component Analyses of all samples based on the plant and soil chemical composition. Different symbol shape indicate plant origin (native, congeneric range-expander $(\mathrm{RE}+)$ and unrelated range-expander (RE-)) and colour indicate north (green) and south (orange) range. Symbols indicate centroids and error bars indicate standard error around the centroids. Arrows indicate plant and soil parameters measured and used in this analysis.

\section{Differences in plant and soil nutrients between range-expanders and their related natives}

All plant and soil nutrient parameters measured were significantly different between the four plant genera sampled, with the only exception of soil ammonium (Table 2.1). Furthermore, all parameters were also different between the north and south range (Table 2.1). Nitrogen concentrations in plant leaves and in the soil (both available and total nitrogen), carbon to nitrogen ration in plant leaves and carbon in the soil was higher in the south than in the north range, while the opposite pattern was found for phosphorous in plant leaves and soil. Main effects of origin were weak and only significant for leaf phosphorous content, soil phosphate and soil nitrate (Table 2.1). For these three 
parameters, range-expanding plant species had higher values than natives, even though these effects are not significant in the post hoc test for soil nitrate and phosphate. Both effects of range and plant origin were plant genus-specific, as indicated by the multiple significant interactions of plant genera with range and/or plant origin. Below, differences between range-expanding plant species and their congeneric natives and between the north and the south range are further detailed for each parameter measured and plant genera.

Plant nitrogen (Fig. 2.2): In Centaurea, nitrogen percentage in plant leaves was not significantly different between native and range-expander or between north and south. In the case of Chaerophyllum, leaf nitrogen was higher for the native species than the rangeexpander in the South range, while there were no differences in the North. For Geranium and Tragopogon, leaf nitrogen percentage was generally higher in range-expanders than in related natives. However, for Tragopogon this was only significant in the south range and for Geranium this was only significant in the north range.

Carbon to nitrogen (CN) ratio in plant leaves (Fig. 2.2): $\mathrm{CN}$ ratio of range-expander and native Centaurea and Geranium species was not different in any of the two ranges. The rangeexpanding Chaerophyllum had higher $\mathrm{CN}$ ratio than natives in the south, but there was no difference in the north. Tragopogon species had a higher $\mathrm{CN}$ ratio in the north than in the south range, but there was no effect of origin.

Plant phosphorous (Fig. 2.2): Phosphorous in leaves of range-expander and native Centaurea and Geranium were not significantly different in any of the two ranges. Range-expanding Chearophyllum had higher phosphorous percentage than the native in the north range, while there was no difference in the south. Native Tragopogon had higher leaf phosphorous than the range-expander, in both south and north ranges. 

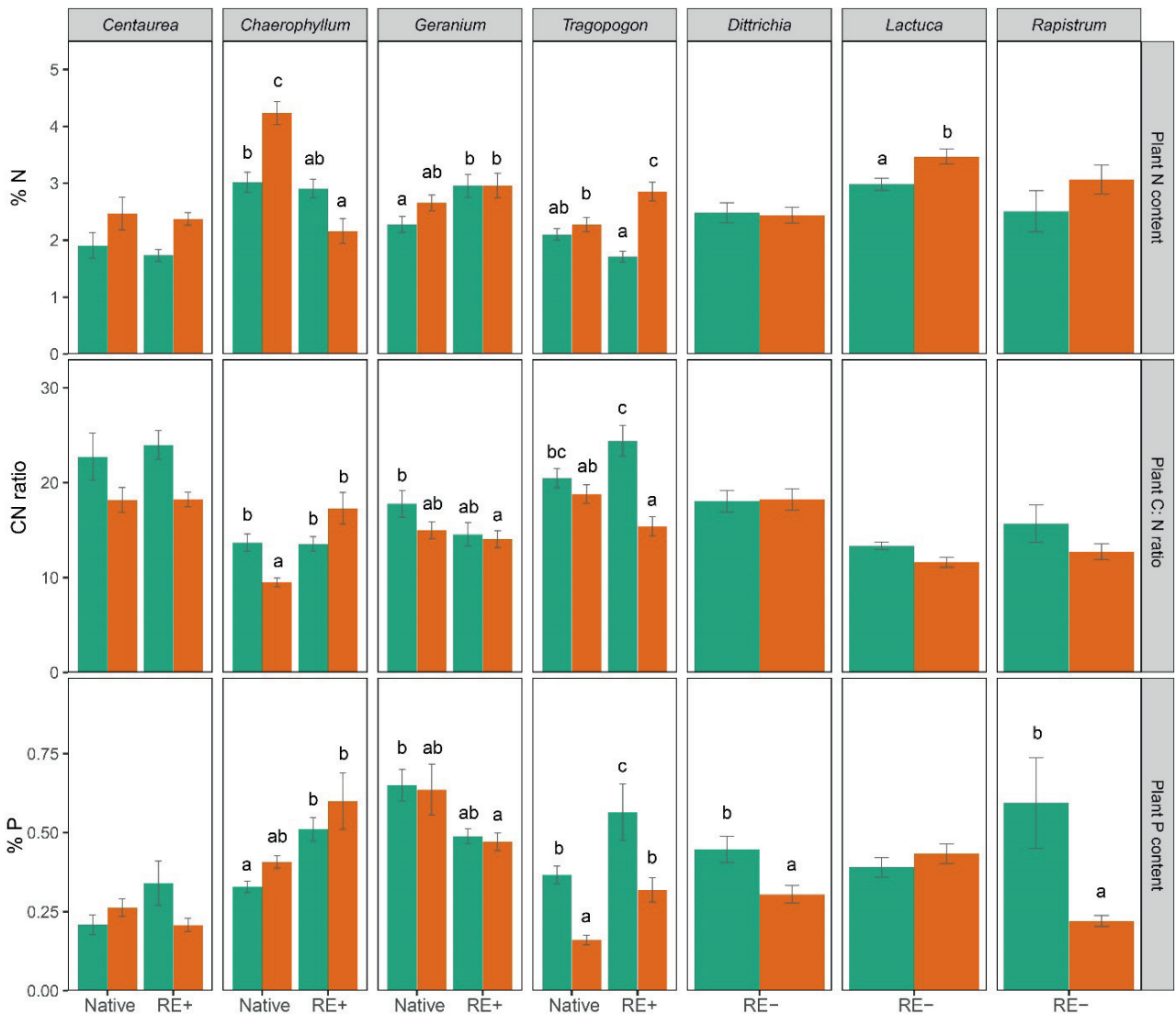

Fig. 2.2. Plant chemical composition (nitrogen content, carbon to nitrogen ratio and phosphorous content in leaves) for native plant species, congeneric range-expanders ( $\mathrm{RE}+$ ) and unrelated rangeexpanders (RE-) within plant genera. Different colours indicate samples belonging to the north range (green) or the south range (orange). Bars are averages and error bars indicate standard error. Sample size of each bar can be found in Table S2.1. Letters above the bars indicate significant differences between samples of different plant origin and range within each plant genera.

Soil nitrate (Fig. 2.3): In Centaurea and Tragopogon, soils of range-expanders had higher nitrate in the south than in the north, while soils of the natives were not different between ranges. Furthermore, there were no significant differences between soils of rangeexpanders and natives. In Chaerophyllum, soil nitrate was not significantly different due to plant origin or range. There was a main effect of range on soil nitrate in the case of 
Geranium, with soils of the south range having highest nitrate availability, but there were no differences between range-expander and native plant species (Fig.3).

Soil ammonium (Fig. 2.3): In soils of Centaurea, ammonium concentration was higher in the south than in the north and in soils of the native than the range-expander. In soils of Chaerophyllum, there was no effect of range within species, but soils of the range-expander had higher ammonium availability than soils of the native. In Geranium, ammonium availability was higher in soils of the range-expander than the native in the south, but not in the north. In the case of Tragopogon, soils in the south had significantly higher phosphate availability than soils in the north. Furthermore, soils of the native species had higher availability of ammonium than soils of range expanders in the north, but not in the south. Soil phosphate (Fig. 2.3): Soil phosphate was neither affected by range nor by plant origin in the case of Centaurea. In Chaerophyllum, soil phosphate availability was higher in the north than the south, but there was no effect of plant origin. For Geranium, soil phosphate availability was higher in soils of the range-expander than the native in both ranges, but there was no difference between ranges. Range-expanding Tragopogon had higher phosphate availability in the soil than the native in the south, but there was no difference in the north.

Soil total carbon (Fig. 2.4): Generally, there were no differences in soil carbon content between soils of natives and range-expanders. Only native Centaurea in the north had more carbon than soils of the range-expander.

Soil total nitrogen (Fig. 2.4): Nitrogen content in the soil was generally higher in the south than the north for all plant pairs, with the exception of Chaerophyllum soils, which were not different. Range-expanding Tragopogon had higher soil nitrogen content than the native in the south, while native Centaurea had higher soil nitrogen content than rangeexpanders in the north. 


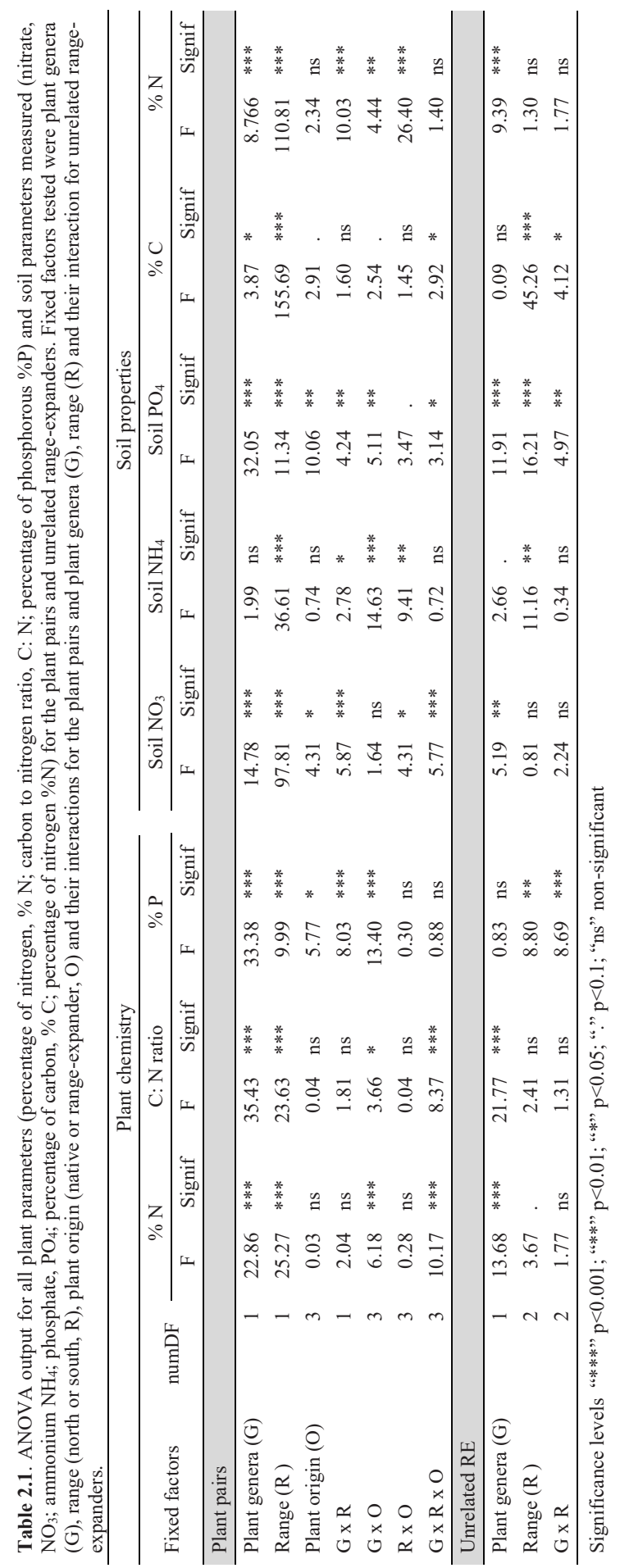




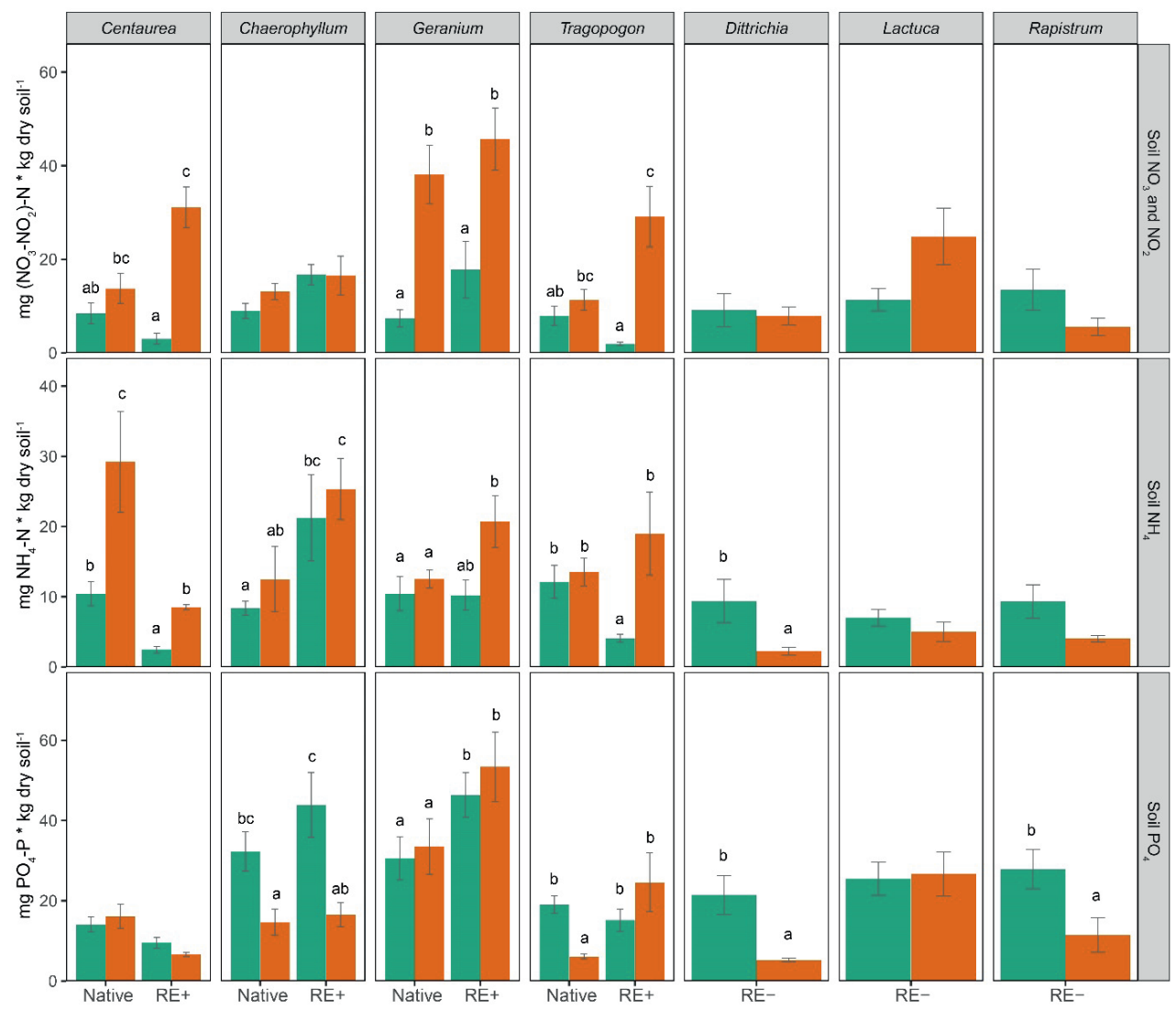

Fig. 2.3. Soil nutrient availability (nitrate and nitrite, ammonium and phosphate) for native plant species, congeneric range-expanders $(\mathrm{RE}+)$ and unrelated range-expanders (RE-) within plant genera. Different colours indicate samples belonging to the north range (green) or the south range (orange). Bars are averages and error bars indicate standard error. Sample size of each bar can be found in Table S2.1. Letters above the bars indicate significant differences between samples of different plant origin and range within each plant genera. 

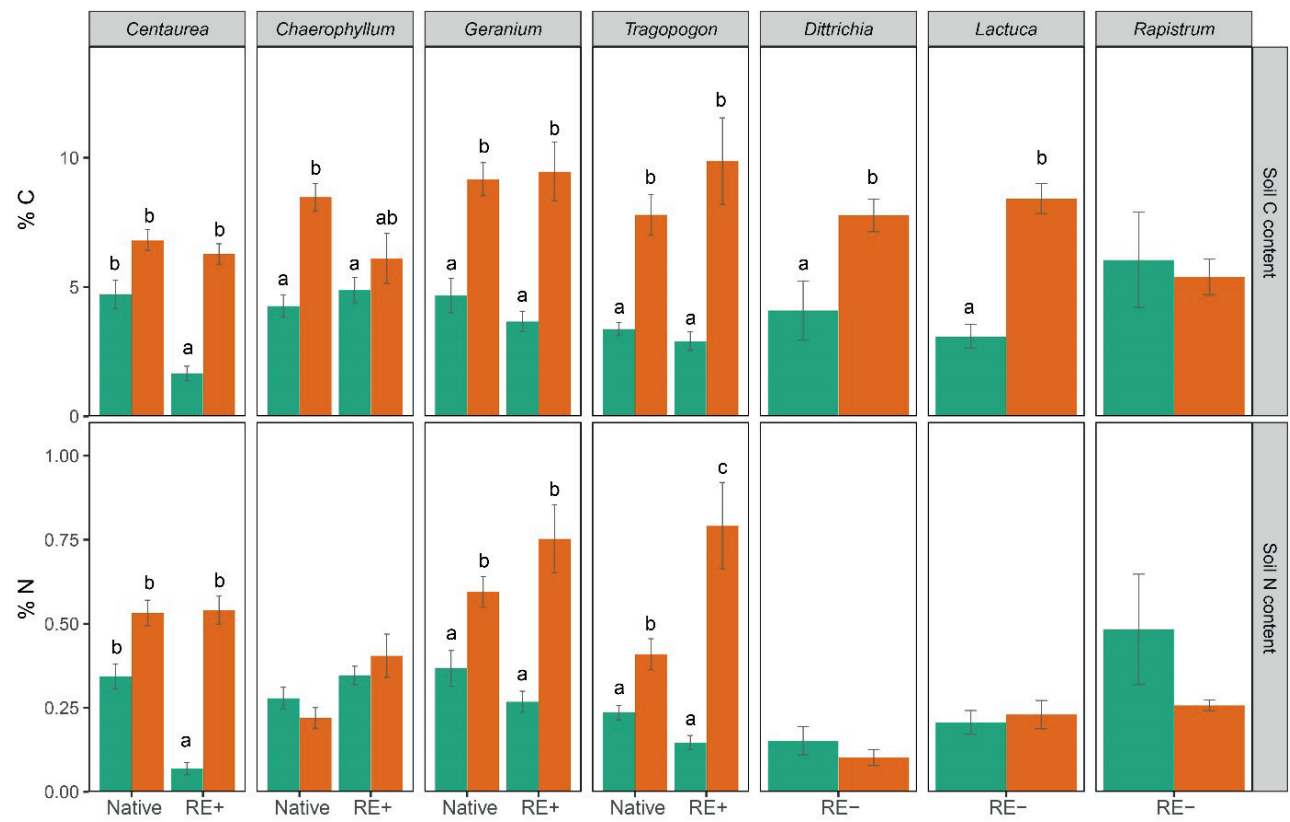

Fig. 2.4. Soil chemical composition (carbon and nitrogen content) for native plant species, congeneric range-expanders (RE+) and unrelated range-expanders (RE-) within plant genera. Different colours indicate samples belonging to the north range (green) or the south range (orange). Bars are averages and error bars indicate standard error. Sample size of each bar can be found in Table S2.1. Letters above the bars indicate significant differences between samples of different plant origin and range within each plant genera.

When examining north and south for each parameter separately, we found that differences between north and south, when present, were also genus specific (Table 2.1). In the case of plant nutrients, nitrogen concentrations in leaves were highest in the south for Lactuca, while there was no difference for Dittrichia and Rapistrum. CN ratio did not differ between north and south for any plant species. Phosphorous was highest in the north range in the case of Dittrichia and Rapistrum (Fig. 2.2), while there was no difference for Lactuca. For soil nutrients (Fig. 2.3), soil nitrate was not different between the north and the south for any plant species. Available ammonium was higher in the north range for Ditricchia, while there were no differences for Lactuca and Rapistrum. In the case of soil phosphate, availability was higher in the north than in the south for both Dittrichia and Rapistrum, which coincided with higher phosphorous amount in plant leaves for these two species, but there were no differences in Lactuca. Finally, soil carbon content was higher in the south than in the north for Dittrichia and Lactuca species, while there were no differences 
for Rapistrum (Fig. 2.4). In terms of nitrogen content, there were no differences between north and south for any of the species (Fig. 2.4).

\section{Discussion}

In this study, we investigated nutrient concentrations in plant leaves and soils of rangeexpanding and native plant species to identify whether non-native species have higher nutrient concentrations in their aboveground plant tissues and soils than natives. Our results show that differences in plant and soil chemistry between range-expanding and congeneric native plant species are genus-specific, and that there is no general relationship between plant species origin (range-expander or native) and plant and soil nutrient concentrations. In addition, differences between range-expanding and native plant species were range-specific as they sometimes only occurred within the north or the south range, without a clear general pattern. Furthermore, plant and soil chemistry within plant species differed between the north and south ranges, and the direction and magnitude of these latitudinal differences were dependent on the parameter and plant species considered.

We found mixed support for our first and second hypotheses that range expanders have higher nutrient content in their leaves and soils than congeneric natives. The main observation from the four plant pairs examined is that native species along the range and species that are native in the south and have expanded their range to the north do not consistently differ in plant and soil nutrient content. This result contrasts with studies of exotic invasive plants showing that exotics generally have higher nutrient concentrations in their shoots and are associated with soils that have higher nutrient availabilities than natives (Pyšek et al. 2012, Sardans et al. 2017). In our study, we did not specifically select range-expanders that are highly invasive, but just species that rapidly increase abundance in the novel range. In contrast, studies on exotic plant species generally focus on those species that become invasive and have the biggest impacts on the recipient ecosystem (Pyšek et al. 2008). However, it has been estimated that the number of exotic introductions is much larger (Williamson and Fitter 1996), and the question is whether all those exotic species also show higher plant and soil nutrient concentrations than natives.

The lack of general effects of plant origin on plant and soil nutrients in the present study may be explained in several ways. A main difference between our study and those on introduced invasive exotics is that we have selected plant species that have expanded their range but that have not been classified as invasive species in the north range (NDFF 2018). 
Therefore, we might miss out species that become really invasive. Furthermore, we compared range-expanding plant species to congeneric natives, which allows disentangling the effect of origin from those of other traits. Consequently, this approach may also decrease variation between compared congeneric species. In most studies on introduced exotics, comparisons are made without accounting for species relatedness, which might results into larger differences between compared exotic and native species (Wolfe and Klironomos 2005, Sardans et al. 2017). The differences observed in those studies, however, may also be due to other characteristics of the species that have not been controlled for, such as life history traits or growth form. Therefore, generalizations about the effect of origin may then be less accurate than in strongly controlled studies, such as the present one, where phylogenetically controlled comparisons are used. Finally, a limitation of our study is that only four plant pairs have been considered, which could have limited possibilities to find strong patterns in the compared plant and soil parameters.

Strauss et al. (2006) suggested that exotic plant species that are less phylogenetically related to natives have a higher chance to becoming invasive. In our study, we did not include a specific test of the role of plant relatedness on plant and soil chemistry. Nevertheless, according to our PCA ordination, unrelated range expanders seem to be more dissimilar from natives than related range-expanders in terms of their plant and soil chemistry (Fig.1). This dissimilarity between unrelated range-expanders and natives is more prominent in the south range, which is the original range of all plant species. This might be due to the initial choice of plant species that was based on their co-occurrence in the north range, where all plant species occurred in the same riverine habitat, whereas in the south the variation of habitats where the species could be found was much wider. This may also explain why variability between samples was lower in the north than in the south range. In order to fully determine the role of plant relatedness in plant range expansion it will be needed to control and test for different levels of relatedness between rangeexpanding and native plant species and to include comparable environmental variation in both the original and new range habitats.

Because nutrient contents of plant leaves of range-expanding species are not generally higher than in plant leaves of natives, it seems unlikely that range-expanders may enhance soil nutrients via their litter inputs as suggested for introduced exotic species (Wardle and Peltzer 2017). Furthermore, from our soil data it also seems unlikely that range-expanders 
preferentially establish in field locations with generally higher nutrient availabilities than native species. Altogether, these results provide limited evidence to conclude whether range-expanding plant species respond to high soil nutrient availabilities or increase them by the input of litter of higher quality. Nevertheless, soil nutrient availabilities differed between ranges indicating that range-expanders show a certain degree of plasticity (Funk 2008). In general, we found no evidence that plant range-expansions can be associated systematically to the introduction of plants with higher nutrient contents or to plants that selectively colonize sites with higher nutrient availability as compared to relative native species.

Making generalizations about what makes certain plant species invasive in a specific environment and finding predictors that explain invasiveness and invasibility remain a general challenge in invasion studies (Hayes and Barry 2008). A main reason for it may be the large diversity of traits of exotic species investigated and also their interactions with the large variability among invaded communities and environments, which also play an important role in determining introduction success (Levine et al. 2004). Plant range expansions provide a novel study system to investigate standing questions in invasion ecology, such as what mechanisms may enable successful introductions, and what may be the functional roles of non-native species in the ecosystems in the new range (van der Putten 2012). Additionally, range-expanders may encounter related species during range expansion, which make them less novel than in case of intercontinental exotics, and allows investigating simultaneously the effect of both origin and plant species relatedness. It is likely that there are plant species that expand their range that do and ones that do not have traits of invasive exotic species. Our data suggest that predicting when and where rangeexpanding plant species may resemble exotic invasive species may be just as challenging as it is for intercontinental exotics. Furthermore, our data suggest that similarity in plant and soil parameters between range-expanding and native plant species may decrease with increasing relatedness between compared plants. We conclude that while there are species-specific differences in plant and soil chemistry and nutrient content between plants that expand their range and their related natives, in general range-expanding plant species do not have higher nutrient contents in plant tissues and associated soil in their novel range. 


\section{Supplementary information}

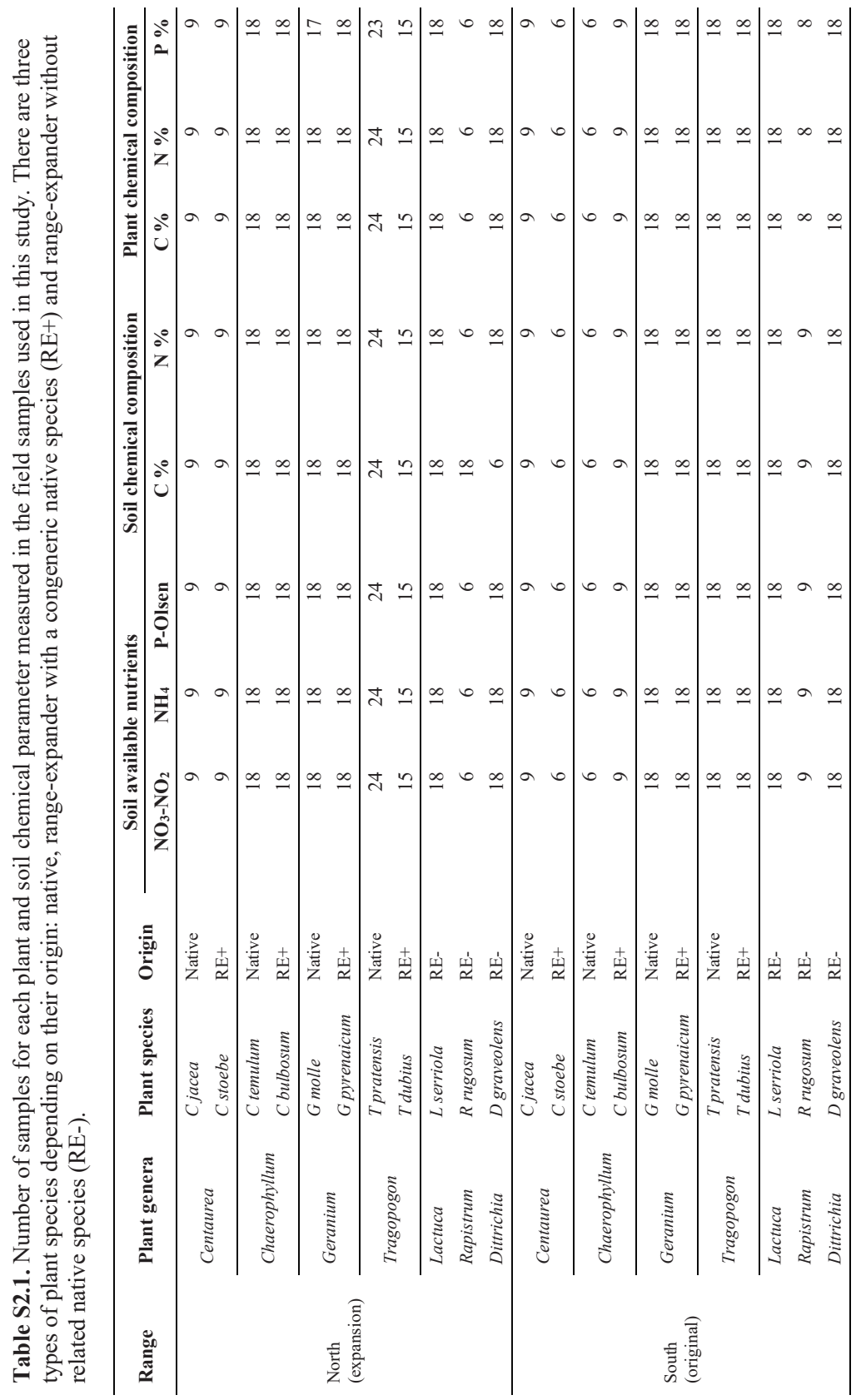


a

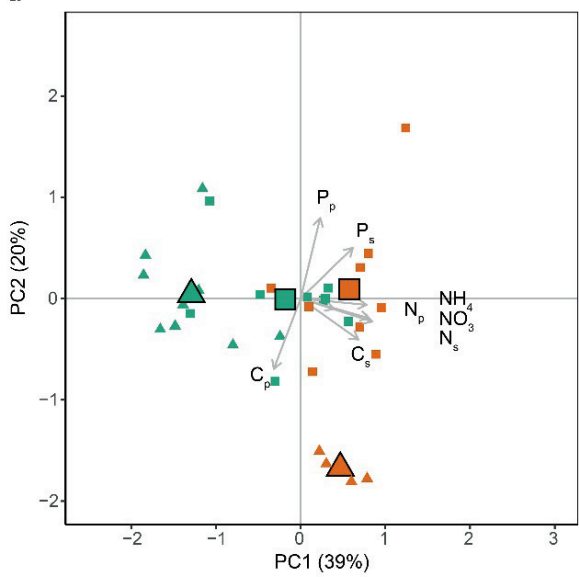

c

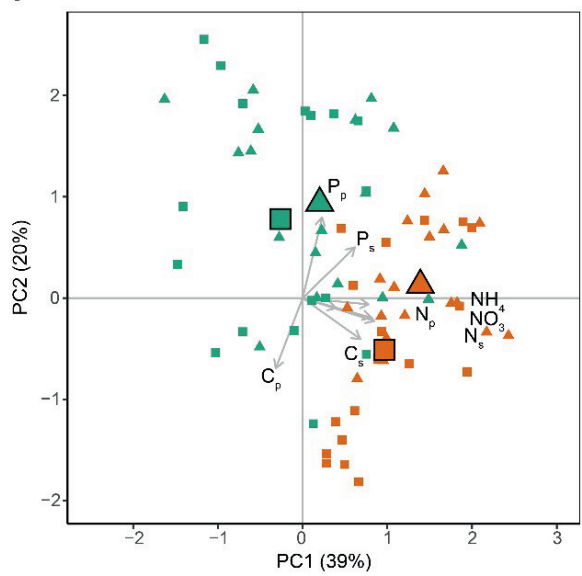

b

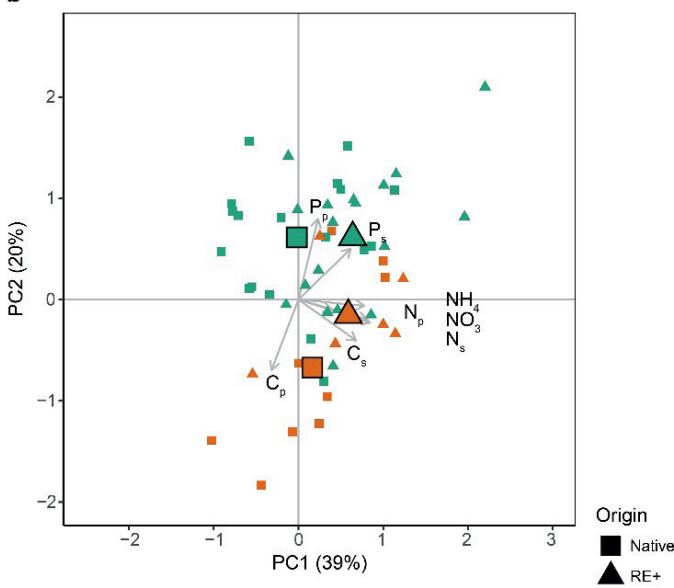

d

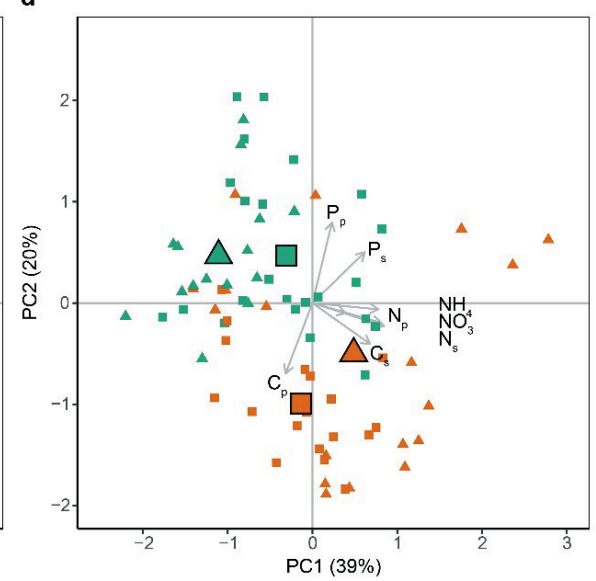

Fig. S2.1. Principal component analyses of all samples based on the plant and soil chemical composition and represented for each of the four plant pairs separately: (a) Centaurea, (b) Chaerophyllum, (c) Geranium and (d) Tragopogon. The shape of the symbol indicates plant origin: native (squares) and congeneric range-expander (triangle). Colour indicates north (green) and south (orange) range. Centroids of each plant origin by range combination are shown next to the individual samples in the ordination plot with bigger symbols. Arrows indicate plant and soil parameters measured and used in this analysis. 
a

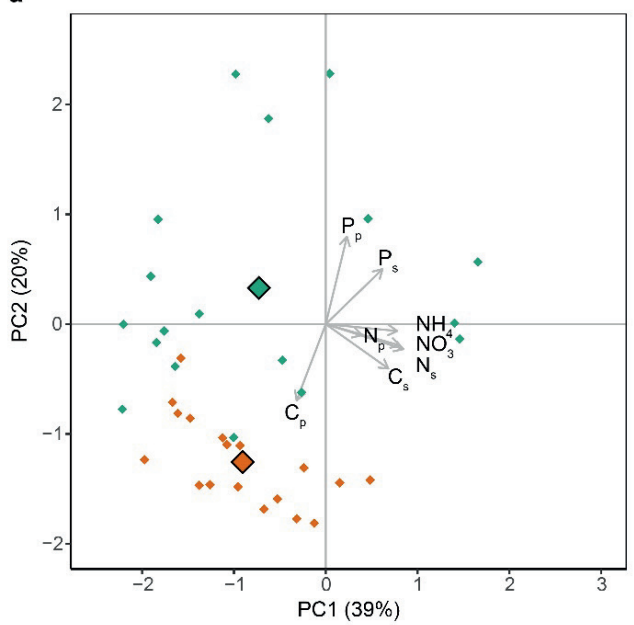

C

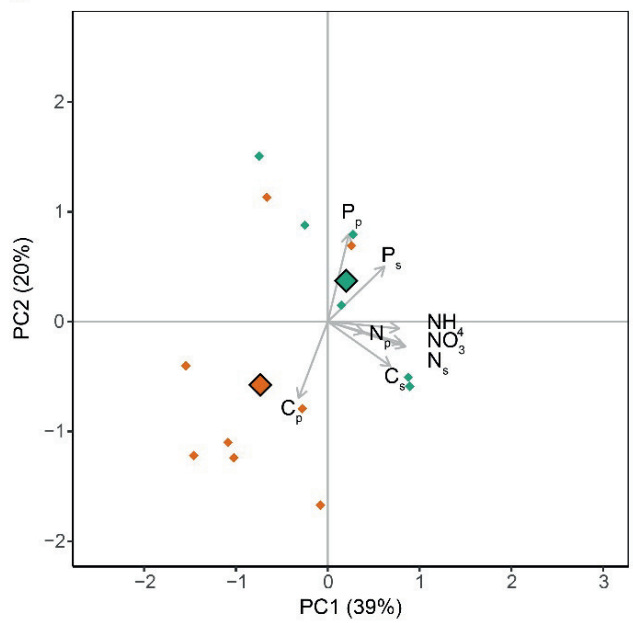

b

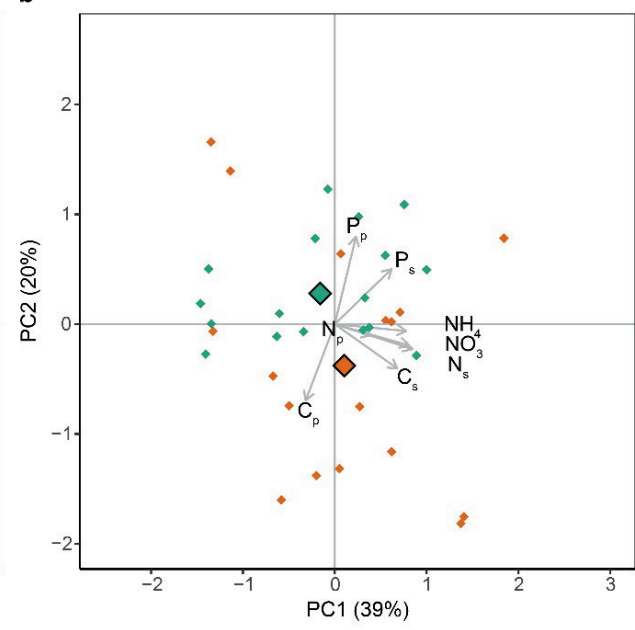

Range

$\checkmark$ North

South

Fig. S2.2. Principal component analyses of all samples based on the plant and soil chemical composition and represented for each of the three unrelated range-expander species separately: (a) Dittrichia graveolens, (b) Lactuca serriola and (c) Rapistrum rugosum. Colour indicates north (green) and south (orange) range. Centroids for each range are shown next to the individual samples in the ordination plot with bigger symbols. Arrows indicate plant and soil parameters measured and used in this analysis. 


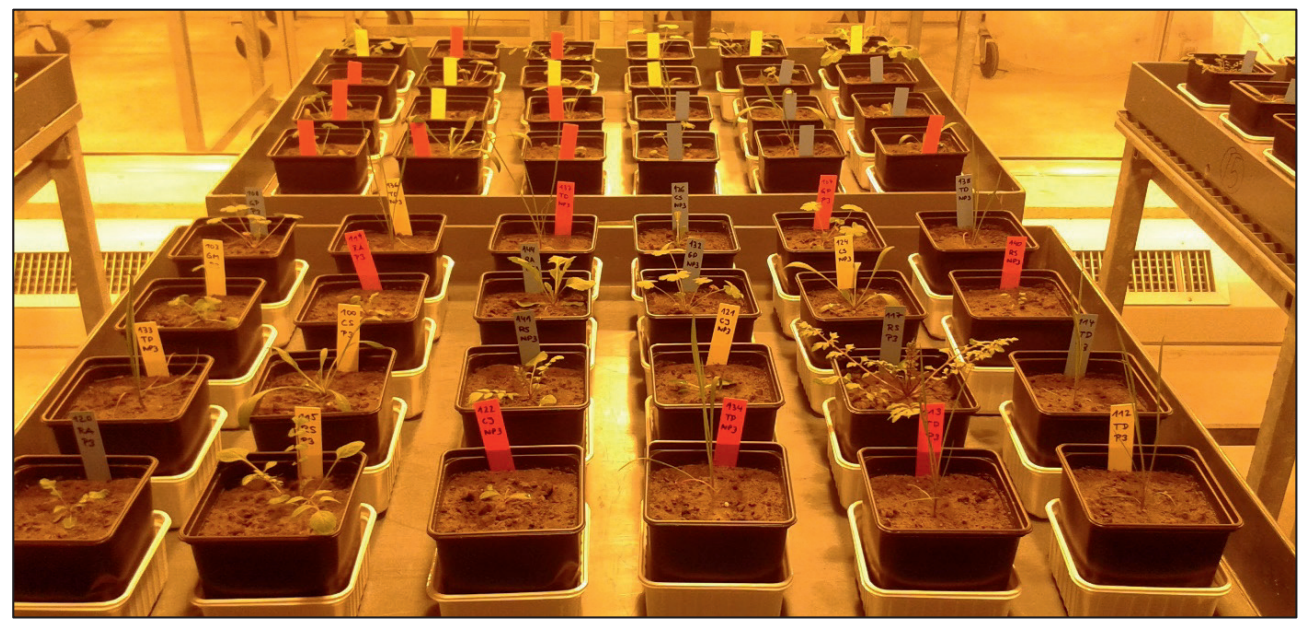




\section{Chapter 3}

\section{Belowground consequences of intracontinental range-expanding plants}

and related natives in novel environments

Marta Manrubia, L. Basten Snoek, Carolin Weser, Ciska Veen \& Wim H. van der Putten 


\begin{abstract}
Introduced exotic plant species that originate from other continents are known to alter soil microbial community composition and nutrient cycling. Plant species that expand range to higher latitudes and altitudes as a consequence of current climate warming might as well affect the composition and functioning of native soil communities in their new range. However, the functional consequences of plant origin have been poorly studied in the case of plant range shifts. Here, we determined rhizosphere bacterial communities of four intracontinental range-expanding plant species in comparison with their four congeneric natives grown in soils collected from underneath those plant species in the field and in soils that are novel to them. We show that, when controlling for both species relatedness and soil characteristics, range-expanding plant species in higher latitude ecosystems will influence soil bacterial community composition and nutrient cycling in a manner similar to congeneric related native species. Our results highlight the importance to include phylogenetically controlled comparisons to disentangle the effect of origin from the effect of contrasting plant traits in the context of exotic plant species.
\end{abstract}




\section{Introduction}

Current climate change is reshaping natural communities by enabling species range expansions to higher altitudes and latitudes (Parmesan and Yohe 2003, Chen et al. 2011, Pauli et al. 2012). Whereas patterns for plants and animals have been relatively well explored, consequences of these range shifts for cryptic species assemblages, such as soil biota, are poorly known (Van Nuland et al. 2017). During range expansion, specific interactions between plants and their co-evolved soil organisms will become disrupted when they have different dispersal capacities (Berg et al. 2010). In the new habitat, rangeexpanding plant species may benefit from the absence of specialized pathogens (Engelkes et al. 2008, Van Grunsven et al. 2010, Morriën et al. 2013, Dostálek et al. 2016). Such enemy release has been documented well for exotic plant species that have been introduced from other continents (Mitchell and Power 2003, Reinhart et al. 2010), and have been proposed to contribute to increased performance of exotics over co-occurring natives (Keane and Crawley 2002, Blumenthal et al. 2009). However, the soil community contains not only pathogens, but also numerous other symbionts and saprophytic microbes that are involved in a variety of ecosystem processes, such as decomposition and nutrient cycling. A key question that is still not well addressed for both introduced exotics and range shifting plant species is how multifunctional soil communities, also including saprophytic microorganisms, may respond to novel host plants with which they lack a co-evolutionary history (van der Putten 2012, Evans et al. 2016).

In the rhizosphere, bacterial community composition is determined by plant species and soil characteristics (Kowalchuk et al. 2002, Berg and Smalla 2009). Saprophytic soil microbes are indirectly affected by plants through the quality and quantity of plant litter and root exudates (De Deyn et al. 2008, Eilers et al. 2010). Novel plant species that have different root exudation patterns or tissue chemistry compared to natives (Macel et al. 2014) will select a specific assemblage of belowground microorganisms (Lankau et al. 2009, Lankau 2011). Depending on the novel plant characteristics, soil communities may shift in their composition and functions when exotic plant species invade (Kourtev et al. 2002a, Wolfe and Klironomos 2005, Vilà et al. 2011, Gibbons et al. 2017, Harkes et al. 2017). However, many studies on belowground functional consequences of exotic invaders are based on comparing species with different traits and life history strategies. Therefore, studies including controls for factors known to influence soil community composition and 
functioning are important to elucidate the effects of species origin (Agrawal et al. 2005, Funk and Vitousek 2007).

Across-species comparison has shown that the invasive potential of exotic species may result from distant relatedness to native plant species, rather than from an effect of geographical origin per se (Strauss et al. 2006). Therefore, identifying functional consequences of ecological novelty of exotic plant species may be more accurate when comparing exotic species with related natives. Several experimental studies have singled out effects of ecological novelty (i.e. plant geographical origin in this case) by comparing exotic plant species with congeneric natives, demonstrating that even when controlling for species relatedness exotics can differ from natives (Agrawal et al. 2005, Funk and Vitousek 2007, Funk and Throop 2010, Meisner et al. 2013b). Here, we aim at understanding how plant species that expand their range to higher latitudes within continents will impact the composition and functioning of the soil bacterial community in the new range as a result of their ecological novelty. We determined the impact of novel range-expanding plant species on native ecosystems in comparison with congeneric natives according to a phylogenetically controlled experimental set up (Engelkes et al. 2008, Meisner et al. 2011, Meisner et al. 2012).

Besides taking into account the relatedness between the compared species, it may also be important to study the effects of plant novelty in the same context. In field studies on plant invasions, it is often difficult to disentangle effects of pre-existing soil communities and abiotic properties from selection effects by plants. In our experiment, we investigated whether potential differences in rhizosphere bacterial community composition between range-expanders and native plant species are the result of selection effects by plants or a response of range-expanders to existing soil heterogeneity. We collected independent replicates of soils from locations in the expanded range where the plant species already occur. In addition, we compared plant effects on bacterial community composition in soils in the expanded range that are new to both range-expanding and native plant species. In this case, differences in rhizosphere bacterial communities are expected to be the consequence of plants selecting for specific bacterial communities. We investigated for both these conditions how range-expanding plant species and congeneric natives develop soil bacterial communities in their rhizosphere, and how this influenced a number of decomposition-related functions. 
In the present study, we compared rhizosphere bacterial communities of range-expanding plant species and their related natives in two different contexts; grown in soils from their own field populations and in soils where both are ecologically novel. Because such differences may depend on plant ontogeny, we repeated our assessments in a time series, so that we could control for differences that may result from plant development. Our first hypothesis was that rhizosphere community composition of range-expanding plants differs from related natives when plants grow in their "own" field soils. Our second hypothesis was that plant origin-specific differences in bacterial rhizosphere communities increase over time when plants are grown in "novel" soils, as that would reveal plant selection effects on soil communities determined by their geographical origin. We assessed functional consequences of differences in soil community composition by measuring catabolic response profiles and soil enzymatic activities. We tested our hypotheses using a controlled greenhouse experiment with four pairs of range-expanding plant species and congeneric natives. Each plant species was grown in "own" and "novel" soils. We determined bacterial community composition and community-level functioning in the rhizosphere of all plants after four, eight and twelve weeks of plant growth.

\section{Materials \& Methods}

Plant species selection and seed origin

We used four pairs of range-expanding and congeneric native plant species and all eight species co-occur in riverine habitat of the Netherlands (Table S3.1). This riveraccompanying ecosystem is connected to Central Europe through the Rhine river, and to South-East Europe through the Rhine-Danube canal. In Central and South-East Europe, > $800 \mathrm{~km}$ away from the Netherlands, the range-expanding and congeneric native plant species are all native. The plant species were selected based on the same criteria used in previous studies (Engelkes 2008, Meisner 2011) (Engelkes et al. 2008, Meisner et al. 2011). Briefly, we selected range-expanding plant species that are present in the Netherlands and co-occur in the same ecosystem with an abundant native plant species of the same genus. The range-expanding plant species were first recorded in the Netherlands during the second half of the $20^{\text {th }}$ century with the exception of Geranium, which was first recorded in $19^{\text {th }}$ century (Dutch flora is very well tracked by many volunteer florists) and show an increasing trend in abundance in the Netherlands over the last decades (NDFF 2018). Because of their co-occurrence in the same riverine habitat and their close phylogenetic (intra-genus) relationship plant species belonging to the same pair differ in their 
geographical origin (i.e. range-expander vs native), but are otherwise quite comparable in genetic background and general ecology.

Seeds of Rorippa species, native Geranium molle, and range-expanders Centaurea stoebe and Tragopogon dubius were collected from the field in the Netherlands. Seeds of native Centaurea jacea, Tragopogon pratensis and the range-expander Geranium pyrenaicum were purchased from an external supplier that collects and propagates seeds from wild plant populations (Cruydt Hoeck, Nijeberkoop, the Netherlands). All seeds were surface sterilized ( $3 \mathrm{~min}, 10 \%$ bleach solution) and germinated on glass beads under controlled conditions (16 h of daylight at $20^{\circ} \mathrm{C}$ and $8 \mathrm{~h}$ of darkness at $10{ }^{\circ} \mathrm{C}$ ). Rorippa seeds were not surface sterilized due to their small size and were germinated in gamma-sterilized soil (minimally 25 KGray, Syngenta BV, Ede, the Netherlands).

Soil collection

During August-October 2015, we collected soils from five independent plant populations of each plant species in order to act as soil inocula in our experiment (Table S3.1). Even though the plant species of interest occurred in mixed plant communities, soils were collected from underneath individuals of the species of interest in each of the five populations. Soil samples were collected from locations that were at least 60 meters apart from each other (Table S3.1). The soils were kept separate as five experimental replicates throughout the experiment. Field soils were sieved using a $4 \mathrm{~mm}$ mesh size to remove coarse elements and stored at $4{ }^{\circ} \mathrm{C}$ in the dark until the experiment started. A subsample of each soil was stored at $-80{ }^{\circ} \mathrm{C}$ for further molecular analyses of the soil microbial community. A second subsample was oven dried at $40{ }^{\circ} \mathrm{C}$ for five days in order to determine moisture content and soil C: $\mathrm{N}$ ratio using an elemental analyzer (Flash EA 1112, Thermo Fisher Scientific Inc., Waltham, USA). Soil available phosphate (P-Olsen) was extracted in a $0.5 \mathrm{M} \mathrm{NaHCO} 3$ solution and quantified using an autoanalyzer (QuAAtro Autoanalyzer, SEAL Analytical Ltd., Southampton, UK). Finally, we extracted available $\mathrm{N}$ (nitrate and ammonia) from field moist soils by shaking a $10 \mathrm{~g}$ dry weight equivalent in a $50 \mathrm{ml}$ of $1 \mathrm{M} \mathrm{KCl}$ solution for 2 hours. We measured soil $\mathrm{pH}$ in the $\mathrm{KCl}$ extracts and determined the concentration of mineral nitrogen $\left(\mathrm{NH}_{4}^{+}\right.$and $\left.\mathrm{NO}_{3}{ }^{-}-\mathrm{NO}_{2}{ }^{-}\right)$using an autoanalyzer (QuAAtro Autoanalyzer, SEAL Analytical Ltd., Southampton, UK).

In our experiment, we inoculated a sterilized background soil with living (non-sterilized) field soil. This method is commonly used and allows studying plant responses to soil biota while controlling for potential differences in abiotic properties of the soils (Engelkes et al. 
2008). Background soil was collected from a riparian area near Beneden-Leeuwen, the Netherlands (N5153.952, E05³3.670). Background soil was sieved using a $1 \mathrm{~cm}$ mesh size, homogenized and gamma-sterilized (minimally 25 KGray, Syngenta BV, Ede, the Netherlands).

\section{Experimental setup}

We inoculated the sterilized background soil with 10\% of live field soil (dry weight basis). For each plant species we used two different types of soil inocula, i.e., one type from replicate sites where the plant species was present in the field ("own soils") and one type from replicate sites representing a novel soil inoculum where neither that plant species nor its related congener were present ("novel soils"). Each novel soil replicate was created by mixing one replicate of the soils of all non-congeneric species using equal amounts on a dry weight basis. The novel soil of each replicate was split into two halves, one for growing the range expander and the other for growing the congeneric native. Therefore, the "novel" soil mixes also originated from habitats within the riverine ecosystem where plant species could occur, but had not been previously conditioned by the plant species grown in the experiment.

Pots of 1.1-liter were filled with the equivalent of $850 \mathrm{~g}$ of dry weight of soil. We adjusted soil moisture to $60 \%$ of the soil water holding capacity and kept it constant during the experiment by watering two times per week to re-set weight. Pots with soil only were preincubated in the greenhouse for 4 days in order to adjust to the water content and allow the inoculated soil microbial communities to establish in the sterilized soil. Afterwards, one seedling of each plant species was planted in the pots. During the first week, we replaced the seedlings that failed to establish. Greenhouse conditions were controlled to $16 \mathrm{~h}$ day length with day temperature of $21^{\circ} \mathrm{C}$, night temperature of $16^{\circ} \mathrm{C}$ and average air humidity of $60 \%$. Artificial light was supplied when required (High pressure Sodium (Son-T, 600W Philips GP)).

We destructively sampled rhizosphere soil at 3 different time points during plant development and measured plant biomass after 4, 8 and 12 weeks since the start of the experiment. Pots were organized in a randomized block design in the greenhouse with 5 replicate blocks. In total, 240 pots were set up ( 8 plant species $\times 2$ soil treatments ("own" and "novel") $\times 3$ time points $\times 5$ replicates).

Soil and plant biomass sampling 
At each sampling time, we destructively harvested 80 pots (8 plant species $\times 2$ soil treatments ("own" and "novel") $\times 5$ replicates). First, we removed the whole plant and soil from the pot. Then, the top soil in the pots and the soil attached loosely to plant roots was separated and discarded. Finally, roots were shaken vigorously and the soil that detached last from the roots was considered the "rhizosphere soil". We filled an Eppendorf tube with that rhizosphere soil and stored it at $-80{ }^{\circ} \mathrm{C}$ for further molecular analyses of the bacterial community composition. We used the remaining rhizosphere soil to analyze soil community functioning. Plant roots and shoots were separated, roots were washed and above and belowground plant biomass was measured after oven drying to constant weight at $70{ }^{\circ} \mathrm{C}$ for $48 \mathrm{~h}$ (data not shown).

DNA extraction and community-level sequencing analyses

We extracted DNA from all soil samples and subsequently amplified the 16S rRNA gene to determine bacterial community composition. PCR amplicons were sequenced using Illumina MiSeq platform. Details can be found in the Supplementary Information (Materials and Methods).

Community-level functioning of rhizosphere microbial communities

The functional capacities of the soil microbial communities were analyzed by measuring their catabolic response profile (CRP) at each time point of harvesting (after 4, 8 and 12 weeks). Hydrolytic enzyme activity was measured for the last time point (after 12 weeks). Details are presented in the Supplementary Information (Materials and Methods).

Statistical analyses

Abiotic properties of field inocula soils were analyzed with 2-way ANOVA in R ( R Core Team 2017). We tested the effect of plant genera and plant origin on each of the soil abiotic parameters. We considered plant genus as a fixed factor and not random since we selected the genera available after accounting for our selection criteria (Engelkes et al. 2008, Meisner et al. 2011). We tested the effect of plant origin within each plant genus for each of the soil parameters using post-hoc comparisons of least square means with Tukey adjustment. Data was transformed prior analyses to meet assumptions of normality using Box-Cox power transformation for linear models in R (R Core Team 2017).

Canoco 5 software was used to conduct multivariate statistics on bacterial community composition of field soils used as inoculum, and on compositional and functional (CRP) 
data of rhizosphere bacterial communities (Ter Braak and Šmilauer 2012). Relative abundances of bacterial OTUs in soil communities and on soil respiration responses were $\log$ transformed prior the analyses. We performed Principal Coordinate Analyses (PCoA) of the dissimilarity matrix based on Bray-Curtis distances to visualize differences in bacterial community composition and soil functioning between our treatments. For the inocula soils, soil abiotic properties measured ( $\mathrm{pH}, \mathrm{C}: \mathrm{N}$ ratio, nitrate, ammonia and plantavailable phosphate) were projected as supplementary variables in the PCoA ordination. Furthermore, we statistically tested the effect of soil parameters and plant species on bacterial community composition of the inocula soils using PERMANOVA (999 permutations) (Oksanen et al. 2018). As explained earlier in the Methods section, during the experiment, each plant pair formed by a native and range-expanding plant species was grown in novel soils created by mixing the soils from the replicate sites of the noncongeneric plant species. Thereby, the novel soils were different from one plant pair to another and, for this reason data analyses were performed for each plant pair separately. For the same reason, we also conducted the analyses in "novel" and "own" soils per plant pair. The analyses within "novel" and "own" soils allowed us to zoom in on the plantdriven variation in rhizosphere communities and their functions in the case of novel soils, and examine differences in more detail in the case of own soils. In all cases, block was included as a covariate in the analyses. To statistically test the significance of plant origin, soil inocula and time of harvest effects on community composition and functioning, we performed PERMANOVA analyses using the 'adonis' function in the 'vegan' package in R (Oksanen et al. 2018). Permutation tests (9999 permutations) were performed within each plant pair and we tested the effect of individual and interaction effects of plant origin, soil inocula and time of soil conditioning by the plants. We performed multivariate dispersion analyses (999 permutations) to test for homogeneity of dispersion between the different plant origin, soil and time point groups and, thereby, validate the PERMANOVA tests (Anderson 2006). Homogeneity of dispersion was measured using the 'betadisper' function in the "vegan" package (Oksanen et al. 2018).

Bacterial OTU richness and Shannon's diversity indices of bacterial communities $\left(\mathrm{H}^{\prime}\right)$ and evenness were computed for each sample using Canoco 5 (Ter Braak and Šmilauer 2012). We analyzed the community parameters in inocula soils following the same way as the abiotic soil parameters described above. For the experimental soils, we analyzed community parameters with linear mixed models using "lmerTest" package (Kuznetsova et al. 2013). We modeled community parameters for each of the three time points 
separately with plant genus, plant origin and soil as fixed effect factors and block as random factor. All analyses were conducted in R (R Core Team 2017). OTU richness was $\log$ transformed prior to analyses. Diversity and evenness data were transformed prior to analyses to meet assumptions of normality using Box-Cox transformation for linear models in R.

Additionally, the effects of plant origin and soil inocula on soil enzyme activity were tested using linear mixed models with plant genus, plant origin (native or range expander) and soil inocula ("own" or "novel") as fixed effect factors, and block as a random factor. Enzyme activity rates were log transformed prior to analyses to meet assumptions of normality. All analyses were conducted in R (R Core Team 2017). 


\section{Results}

Bacterial community in field soils (inocula soils)

The variation of bacterial community composition represented by the first two axes of the PCoA was $39 \%$ of the total variation (Fig. S3.1). Soil abiotics and plant species identity explained 40 and $21 \%$ of the variation in soil bacterial communities, respectively, as tested with PERMANOVA (Table S3.2). Bacterial communities in soils of native and rangeexpanding Geranium species were more similar to each other than for the other plant pairs. However, soil bacterial communities associated with the natives Centaurea jacea and Tragopogon pratensis were more similar to each other than to their related range-expanders Centaurea stoebe and Tragopogon dubius (Fig. S3.1). Soil bacterial communities of Rorippa species were most different from the rest and were associated to soils with higher soil $\mathrm{pH}$ and C: N ratio compared to the other plant species (Fig. S3.1, Fig. S3.2, Table S3.3). Overall, bacterial community richness, diversity and evenness was affected by plant genus, yet no significant effect was found for plant origin (Table S3.3, Fig. S3.2). Soil nitrate availability was significantly higher in soils of native plant species than in soils of range expanders. In the case of ammonia, soils of native species had lower ammonia then soils of rangeexpanders, with the exception of the Tragopogon species (Table 3.2, Fig. S3.2).

\section{Bacterial community composition in the rhizosphere of the experimental plants}

The variation of bacterial community composition represented by the first two axes of the PCoA accounted for $18 \%, 16 \%, 25 \%$ and $36 \%$ of the total variation in Centaurea, Geranium, Tragopogon and Rorippa species, respectively (Fig. 3.1). Soil inocula ("novel" or "own") was the most important factor explaining variation in bacterial community composition in all plant pairs with the exception of the Tragopogon pair, where soil inocula and plant origin explained the same amount of variation (Table 3.1). The interaction of plant origin and soil inoculum explained bacterial community in the rhizosphere in all plant pairs except in Geranium (Table 3.1). Overall, bacterial communities were separated between native and range expanders when they were grown in their "own" field soils, but did not differ when grown in "novel" soils. Multivariate dispersion analyses indicated that dispersion within groups was homogeneous between range-expanders and natives and between time points for each of the 4 plant pairs. However, "own" soils had significantly higher variation than "novel" soils in Geranium, Tragopogon and Rorippa plant pairs (F 8.8 p 0.004, F 58.2 p 0.001 and F $28.8 p$ 0.001, respectively). This indicated that community composition was more 
similar between samples of "novel" soils, while there was more variation between samples in "own" soils.
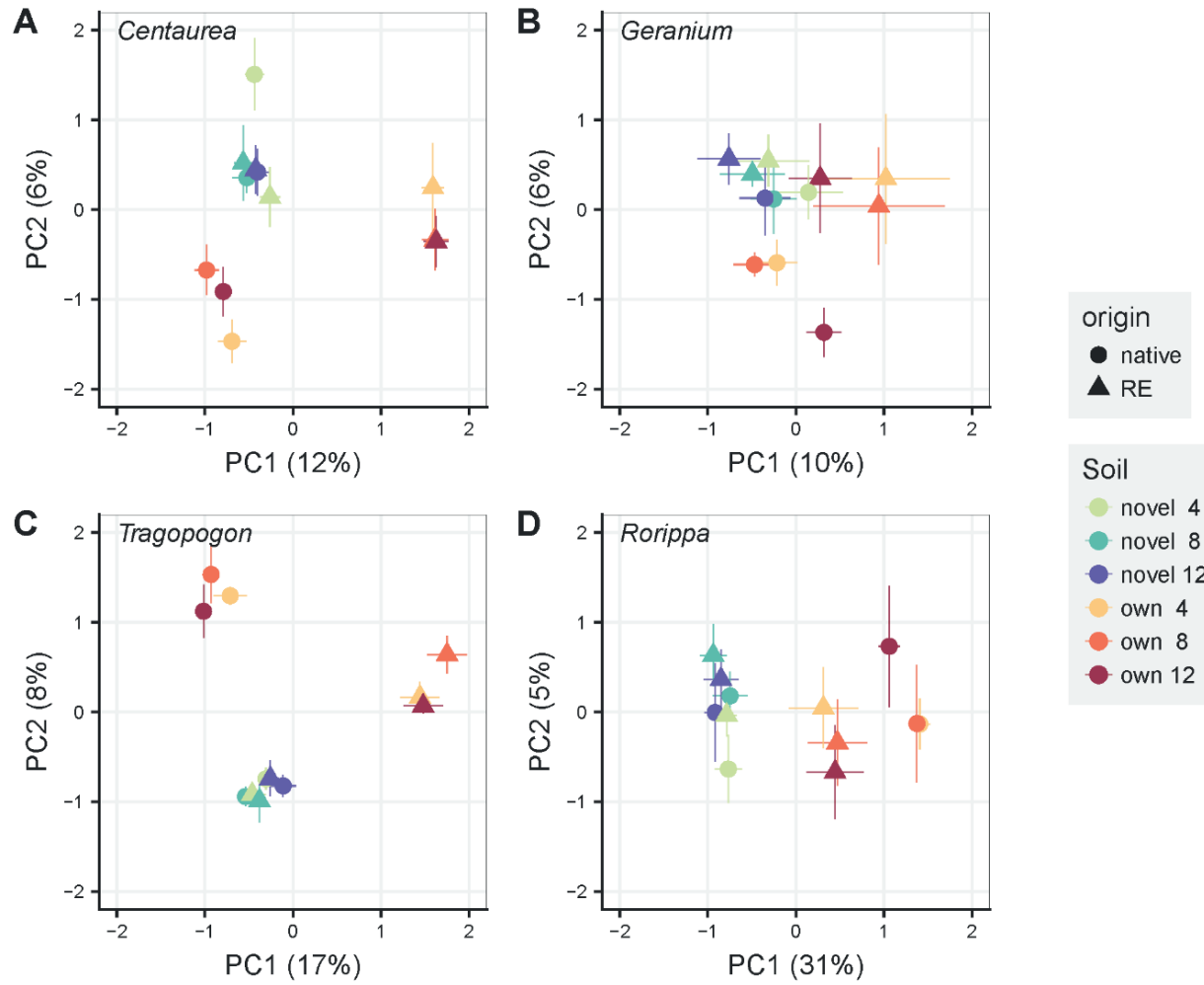

Fig. 3.1. Principal Coordinate Analyses of the rhizosphere bacterial community composition for each pair of a range-expander and its congeneric native plant species (A: Centaurea, B: Geranium, C: Tragopogon, D: Rorippa) grown in soils from their own field locations (own) and soils that are novel to both of them (novel). The symbols are means $\pm \mathrm{SE}(\mathrm{N}=5)$. Within each pair, circles represent the native plant species and triangles the range-expander. Colours indicate soil treatment ("own" and "novel") and time of harvest (4, 8 and 12 weeks) as noted in the legend.

To test the effect of plant origin more accurately we performed the same analyses within each soil treatment, which allows to disentangle the effect of plant origin from the effect of pre-existing differences in soil bacterial communities. We then observed that, in "novel" soils, plant origin no longer explained variation in bacterial community composition (Fig. S3.3, Table S3.5). Instead, time of harvest appeared to explain around $10 \%$ of the total variation in Geranium and Tragopogon plant pairs (Table S3.5). In "own" soils, i.e. originating from field populations of each species, plant origin significantly explained 
bacterial community composition at the end of the growth experiment (Fig. S3.4, Table S3.5). In both "novel" and "own" soils, the variation in community composition of the samples belonging to range-expanders and natives and to the different time points showed homogeneous dispersion, indicating that the variation in community composition was equal between the groups. There was only the exception of the Geranium plant pair grown in "own" soil, where soils of range-expanders had higher variation in community composition than soils of natives (F 5.8 p 0.02).

Soil treatments ("own" and "novel") significantly differed in their bacterial richness and diversity at all sampling times (Table S3.3; Fixed factors, Soil). Novel soils, which were mixes of all soil samples collected from field sites with non-congeneric plant species, had significantly higher richness and diversity of bacterial OTUs than own soils, which originated from individual locations where the tested plant species were present in the field. Bacterial communities in own soils of Rorippa species were most different from the other plant pairs (Fig. S3.2, Table S3.4) and also from their novel soil, which is represented by a significant soil and plant genera interaction. Overall, richness, diversity and evenness of rhizosphere bacterial communities were not significantly affected by plant origin itself (Table S3.4).

Table 3.1. PERMANOVA tests on Bray-Curtis dissimilarity matrix ( 9999 permutations)

\begin{tabular}{|c|c|c|c|c|c|c|c|c|c|}
\hline & \multirow[b]{2}{*}{ Factor } & \multicolumn{2}{|c|}{ Centaurea } & \multicolumn{2}{|c|}{ Geranium } & \multicolumn{2}{|c|}{ Tragopogon } & \multicolumn{2}{|c|}{ Rorippa } \\
\hline & & $\mathrm{R}^{2}$ & Signif. & $\mathrm{R}^{2}$ & Signif. & $\mathrm{R}^{2}$ & Signif. & $\mathrm{R}^{2}$ & Signif. \\
\hline \multirow[t]{7}{*}{$\begin{array}{l}\text { Bacterial } \\
\text { community }\end{array}$} & Plant origin $(\mathrm{P})$ & 0.057 & $* * *$ & 0.036 & $* *$ & 0.082 & $* * *$ & 0.030 & * \\
\hline & Soil inocula (S) & 0.067 & $* * *$ & 0.049 & $* * *$ & 0.082 & $* * *$ & 0.199 & $* * *$ \\
\hline & Time $(\mathrm{T})$ & 0.050 & $* *$ & 0.054 & $* *$ & 0.047 & $*$ & 0.040 & $\mathrm{~ns}$ \\
\hline & $\mathrm{P} * \mathrm{~S}$ & 0.055 & $* * *$ & 0.023 & ns & 0.081 & $* * *$ & 0.026 & $*$ \\
\hline & $\mathrm{P} * \mathrm{~T}$ & 0.020 & ns & 0.024 & ns & 0.022 & ns & 0.018 & ns \\
\hline & $\mathrm{S} * \mathrm{~T}$ & 0.022 & ns & 0.021 & ns & 0.021 & ns & 0.023 & ns \\
\hline & $\mathrm{P} * \mathrm{~S} * \mathrm{~T}$ & 0.019 & ns & 0.019 & ns & 0.023 & ns & 0.016 & ns \\
\hline \multirow[t]{7}{*}{$\begin{array}{l}\text { Community } \\
\text { functioning }\end{array}$} & Plant origin $(\mathrm{P})$ & 0.003 & ns & 0.001 & ns & 0.001 & ns & 0.000 & ns \\
\hline & Soil inocula (S) & 0.031 & ns & 0.028 & ns & 0.028 & ns & 0.028 & ns \\
\hline & Time $(\mathrm{T})$ & 0.009 & ns & 0.005 & ns & 0.009 & ns & 0.010 & ns \\
\hline & $\mathrm{P} * \mathrm{~S}$ & 0.002 & ns & 0.001 & ns & 0.000 & ns & 0.000 & ns \\
\hline & $\mathrm{P} * \mathrm{~T}$ & 0.001 & ns & 0.000 & ns & 0.000 & ns & 0.001 & ns \\
\hline & $\mathrm{S} * \mathrm{~T}$ & 0.000 & ns & -0.001 & ns & -0.001 & ns & 0.000 & ns \\
\hline & $\mathrm{P} * \mathrm{~S} * \mathrm{~T}$ & 0.001 & ns & 0.000 & ns & 0.000 & ns & 0.001 & ns \\
\hline
\end{tabular}

Significance levels: $n \mathrm{p}>0.05 ;{ }^{*} \mathrm{p}<0.05 ; * * \mathrm{p}<0.01 ; * * * \mathrm{p}<0.001 ; * * * * \mathrm{p}<0.0001$ 


\section{Community-level functional analyses}

We used PCoA ordination to assess differences in the functional responses of soil communities to the various added organic substrates (catabolic response profile). The first and second axis represented $75 \%, 68 \%, 57 \%$ and $55 \%$ of the variation in catabolic response profiles of Centaurea, Geranium, Tragopogon and Rorippa soil communities, respectively (Fig. 3.2). Even though time seems to drive dissimilarity in community functioning in the ordination PCoA plots (Fig. 3.2), none of the experimental treatments (plant origin, soil inocula, time of harvest) explained differences in community level functioning (Table 3.1). Similarly, when functioning of the "novel" or "own" soils were examined separately, neither plant origin nor time of harvest explained the variation in community level functioning (Fig. S3.5, Fig. S3.6, Table S3.5). Overall, compositional differences in bacterial communities were not consistently linked to shifts in catabolic response profiles in our experiment. Multivariate dispersion analyses for community functioning showed that variation among functional profiles was not significantly different between groups of samples with the same plant origin, soil inocula type and across time points.

To study soil functions related to nutrient cycling, the activity of three extracellular enzymes was measured in the rhizosphere soil collected after 12 weeks of plant growth (Table 3.2). There were no main effects of plant origin, indicating that both rangeexpanders and related native species were associated with the same levels of enzyme activity in their rhizosphere soil. Plant genus marginally affected glucosidase enzyme activity in the soil ( $F_{3,60} 2.91, p$ 0.04); however, post hoc testing using Tukey HSD did not yield significant differences between any specific group. Soil treatment ("own" and "novel") significantly affected phosphatase activity $\left(F_{1,60} 11.00, p 0.001\right)$, with higher levels of phosphatase activity in "own" than in "novel" soils. 

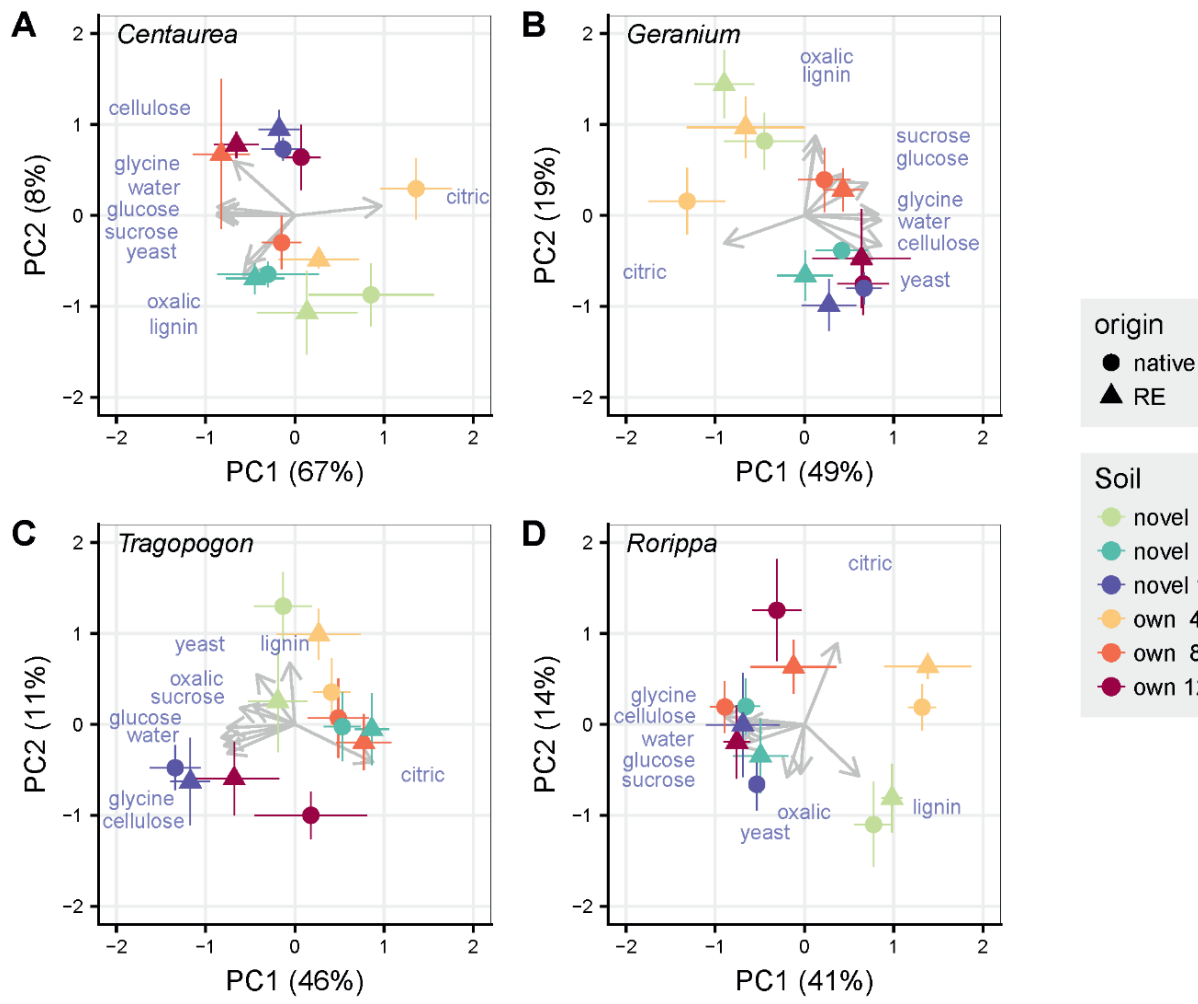

Soil

novel 4

- novel 8

- novel 12

own 4

- own 8

own 12

Fig. 3.2. Principal Coordinate Analyses of the catabolic response profiles for each pair of a rangeexpander and its congeneric native plant species (A: Centaurea, B: Geranium, C: Tragopogon, D: Rorippa) grown in soils from their own field locations (own) and soils that are novel to both of them (novel). The symbols are means $\pm \mathrm{SE}(\mathrm{N}=5)$. Within each pair, circles represent the native plant species and triangles the range-expander. Arrows representing each substrate are displayed over the ordination plot as supplementary variables. Colours indicate soil ("own" and "novel") and time of harvest (4, 8 and 12 weeks) as noted in the legend. 


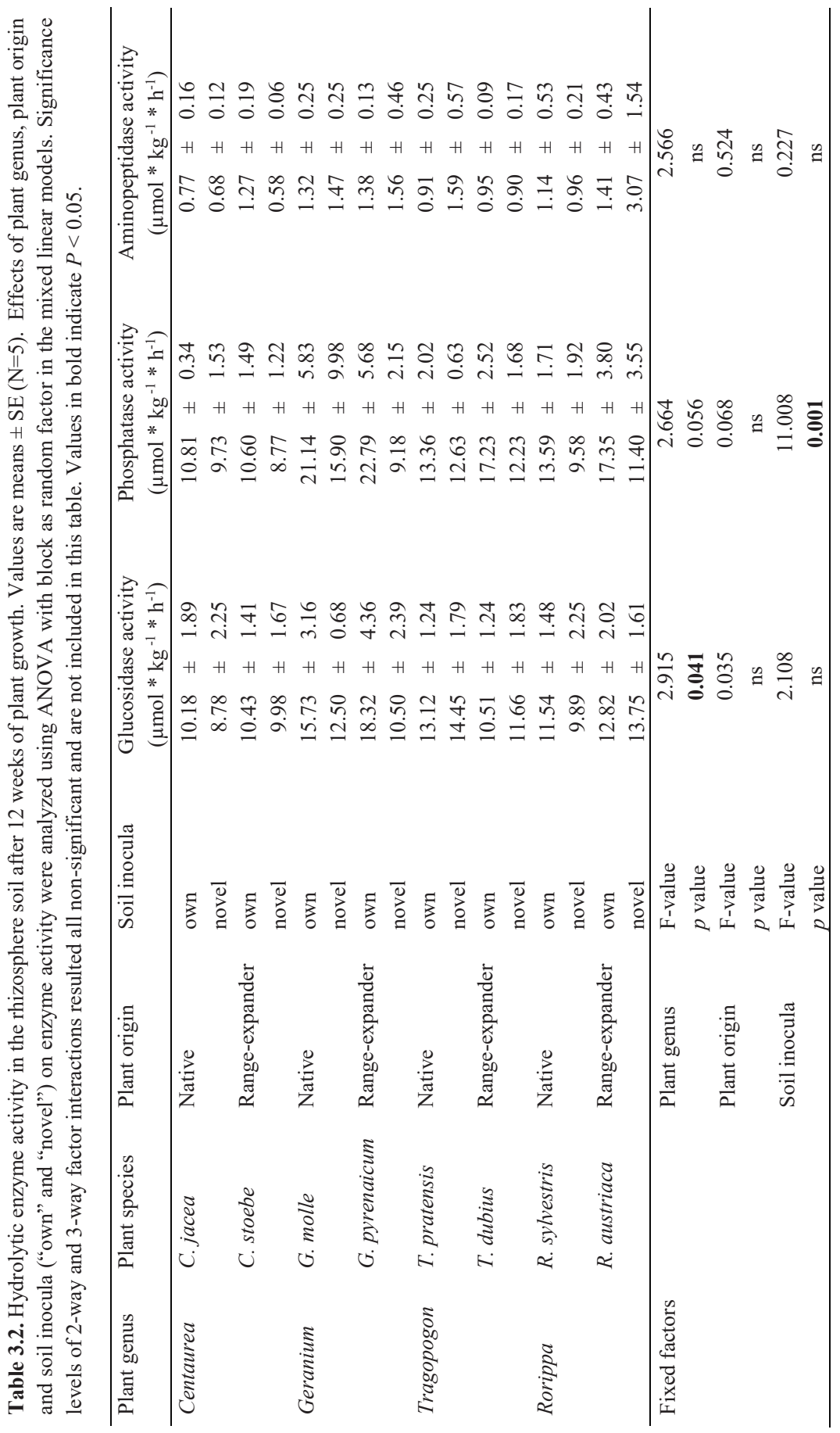




\section{Discussion}

It is generally assumed that exotic plant species may alter composition and functioning of soil microbial communities (Ehrenfeld et al. 2001, Allison et al. 2006, Ehrenfeld 2010). However, in most case studies where exotics are compared with natives, the exotics that replace the natives not only differ in origin, but also in traits or life histories, whereas preinvasion site conditions cannot easily be controlled for (Kourtev et al. 2002a, Wolfe and Klironomos 2005, Vilà et al. 2011). Here, we compare how intra-continental range expanding plant species and congeneric natives influence bacterial community composition and functioning in their rhizosphere, while minimizing genetic differences between range expanders and natives, and controlling for ecological novelty. We paired plant species that expand range most likely as a result of climate warming with species from the same genus that are native in the expansion range (Engelkes et al. 2008, Meisner et al. 2011, Morriën et al. 2013). All plant species were grown in soils collected from established field populations, as well as in soils from sites where neither the range expander nor the native currently occurred. In support of our first hypothesis, we show that rhizosphere bacterial communities differ when growing range expanders and natives in soils collected from their own field populations. Interestingly, when both species were grown in novel soils, there were no compositional differences in rhizosphere bacterial communities. Therefore, and opposite to our second hypothesis, we argue that in the present comparison plant origin per se has little effect on rhizosphere bacterial community composition.

Many field studies on plant invasions and soil communities have shown that exotic plant species have distinct soil communities compared to native plants growing in adjacent areas (Kourtev et al. 2002a, Kourtev et al. 2002b, Scharfy et al. 2009, Collins et al. 2016, Stefanowicz et al. 2016, Gibbons et al. 2017). Consistent with these results, in our experiment we also observe that the composition of the rhizosphere bacterial community differed by plant origin in all four plant pairs when plants were grown in their own field soils (Fig. 3.1, Fig. S3.4). These results suggest that plant origin indeed influences bacterial community composition even in a phylogenetically constrained comparison between range expanders and natives. However, in the present study, as well as in most field studies it is difficult to exclude the possibility that invaded sites were already different from adjacent sites prior arrival of the exotic species. If that is the case, the bacterial assembly in the rhizosphere may simply reflect differences of initial bulk soil communities 
(de Ridder-Duine et al. 2005), or a combination of site and origin differences and, overall, the capacity to disentangle the effect of ecological novelty from other co-varying effects is limited. We tried to rule out such confounding factors as much as possible by growing range-expanding and native plant species also in the same "novel" soil.

In contrast to our second hypothesis, the geographical origin of the plant species (rangeexpanding or native) did not affect rhizosphere bacterial community composition differently when both range-expanding and congeneric native plant species were grown in the same "novel" soils (Fig. 3.1, Fig. S3.3). Our results diverge from previous controlled experiments, which concluded that plant origin may play a role in influencing soil community composition in both intercontinental exotic plant invasion (Kourtev et al. 2003) and plant range-expansion (Morriën et al. 2013). In contrast with many intercontinental exotic plant invasion studies, we used phylogenetically controlled comparisons to assess the effect of plant species origin, while intending to minimize their ecological differences. Consequently, plant species in each plant pair were not expected to differ strongly in e.g. life history and plant functional type, which have been suggested as important biotic predictors of soil community and soil functional shifts (Scharfy et al. 2011, de Vries et al. 2012b, Legay et al. 2014, Lee et al. 2017). Alternatively, the relatively short running time of our experiment ( 3 months) might have limited the divergence of the bacterial communities between plants from different origins, although this running time is not shorter than that of most plant-soil feedback experiments where plants produced different soil microbial community compositions (Heinen et al. 2017). Furthermore, our main interest was to assess shifts in saprophytic microbes in the rhizosphere community and thereby, we analyzed the composition of the whole bacterial community rather than looking at the abundance of specific microbial groups, such as potential plant pathogens (Morriën et al. 2013).

In spite of the substantial differences in bacterial community composition in the rhizosphere of plants growing in soil collected from field populations (Fig. 3.1), we observed limited differences in community functioning (Fig. 3.2, Table 3.2). Previous experiments manipulating the composition of microbial communities have shown high levels of functional redundancy in microbial communities (Franklin and Mills 2006, Wertz et al. 2006). Plant-induced changes in microbial-mediated soil processes may be the result of comparing phylogenetically distant plant species (Ehrenfeld et al. 2001) or major plant community shifts (Carney and Matson 2005). They may also be derived from studies 
focusing on longer time scales (Collins et al. 2016) or on specific soil processes such as nitrogen cycling (Hawkes et al. 2005). Nevertheless, in spite of the possibility that plant selection effects may have influenced soil fungal communities (Dassen et al. 2017, Hannula et al. 2017), it is obvious that those changes, if occurring in our study, have not influenced soil microbial functioning either.

We conclude that intracontinental plant range expansions may lead to populations of novel plant species that have different bacterial communities than congeneric natives, but that this is not necessarily due to their different geographical origin. When range expanding and native plant species from the same genus pair were grown for three months in novel soils, bacterial rhizosphere communities of the range expander and the congeneric native were indistinguishable. Interestingly, the differences in bacterial community composition did not result in altered ecosystem processes as is demonstrated by the respiration of different organic substrates. Therefore, our results demonstrate that plant origin per se does not necessarily have a major impact on bacterial community composition and soil microbial functioning when keeping all other aspects the same. This does not exclude the possibility that range expanders may influence community composition and ecosystem functioning when they are exposed to the soils for longer time periods, or in other ways, such as by responding differently to extreme weather events (Meisner et al. 2013b), natural enemies (Engelkes et al. 2008, Van Grunsven et al. 2010, Dostálek et al. 2016), and other conditions that may typify their novel environments.

\section{Acknowledgements}

We thank Janneke Bloem and Freddy ten Hooven for their practical assistance and guidance during DNA sample preparation. We thank Rosanne van der Linden for her help with enzyme activity measurements. We also thank Hans Zweers and Ciska Raaijmakers for their help with the $\mathrm{CO}_{2}$ measurements using gas chromatography. This study was sponsored by the European Research Council Advanced grant ERC-Adv 260-55290 SPECIALS to Wim H. van der Putten. Ciska Veen was sponsored by NWO-VENI grant (863.14.013).

\section{Data depository}

All sequence data has been uploaded to the European Nucleotide Archive (ENA) under the accession number PRJEB26590. 


\section{Supplementary information}

\section{SI Materials and Methods}

In this section, we provide detailed information about the community-level sequence analyses and functional analyses (catabolic response profiling and extracellular enzyme activity).

Community-level sequence analyses

Eppendorf tubes containing rhizosphere soil were freeze-dried prior to DNA extraction (FreeZone 12, Labconco, Kansas City, USA). DNA was extracted from $0.25 \mathrm{~g}$ of soil using PowerSoil DNA Isolation Kit (Mo Bio Laboratories, Carlsbad, California, USA) following the manufacturer's instructions. We then amplified DNA using duplicate PCR reactions with bar-coded primers. Bacterial community composition was determined by targeting $16 \mathrm{~S}$ rRNA gene using 515F/806R primers (Caporaso et al. 2012). PCR products were purified using the Agencourt AMPure XP magnetic bead system (Beckman Coulter Life Sciences, Indianapolis, Indiana, USA) with a volume of PCR product to beads of 1 to 0.7 . Purified PCR products were analyzed in a Fragment Analyzer using a Standard Sensitivity NGS Fragment Analysis kit (1bp-6000bp) and following manufacturer's instructions (Advanced Analytical Technologies GmbH, Heidelberg, Germany). Finally, bacterial PCR amplicons were sequenced using Illumina MiSeq platform.

The 16S rRNA amplicon reads, MiSeq paired-end reads, were merged when reads had a minimum overlap of $150 \mathrm{bp}$ and at least a PHRED score of 25 using the RDP extension of PANDASeq (Masella et al. 2012) named Assembler (Cole et al. 2014). Primer sequences were removed using Flexbar version 2.5 (Dodt et al. 2012). Sequences were clustered to OTU with VSEARCH version 1.0.10 (Rognes et al. 2016), using the UPARSE strategy by dereplication, sorting by abundance with removing singletons and clustering using the UCLUST smallmem algorithm (Edgar 2010). Chimeric sequences were detected using the UCHIME algorithm. All reads were mapped to OTUs and a OTU Table was created and converted to BIOM-Format 1.3.1 (McDonald et al. 2012). Taxonomic information for each OTU obtained using the RDP Classifier version 2.10 (Cole et al. 2014). All steps where implemented in a workflow made with Snakemake (Köster and Rahmann 2012). Samples with a total sequence number lower than 1000 reads and singleton OTUs (e.g. OTU which is only found once in one sample) were removed from further analyses. 
The sequencing analyses of the 16S rRNA region of all soils yielded an average of 2.761 OTUs per sample $( \pm 944 \mathrm{SD})$, with a total of 5.452 .733 reads. OTUs belonged to 25 different phyla (including bacteria and archaea), 82 classes, 136 orders, 274 families and 630 genera.

Catabolic response profiling

We used a catabolic response profile method as described in Fierer et al. (2012) in order to assess how soil communities differ in their ability to mineralize different organic carbon compounds. For each pot, we measured the $\mathrm{CO}_{2}$ production response of the soil communities after the addition of 8 organic substrates of varying complexity (i.e., glucose, sucrose, glycine, oxalic acid, citric acid, yeast, lignin and cellulose). Organic carbon solutions were made before the soil sampling started and adjusted to a $\mathrm{pH}$ of 6.0. These analyses were carried out immediately after sampling of rhizosphere soil. Briefly, the equivalent of $4 \mathrm{~g}$ of dry soil was weighed into 9 different $50 \mathrm{ml}$ centrifuge tubes. Then, each tube received $8 \mathrm{ml}$ of one of the organic carbon substrate solutions. Additionally, one of the tubes received water as a control. Tubes containing soils and substrates were incubated for $1 \mathrm{~h}$ uncapped in a horizontal shaker $\left(20^{\circ} \mathrm{C}\right)$. Centrifuge tubes were then closed tightly with a modified lid equipped with a rubber septum and a rubber O-ring in order to ensure air tightness. We then flushed the headspace air in the tubes with $\mathrm{CO}_{2}$-free air for 2 min at 1 bar (Westfalen Gassen Nederland BV, Deventer, the Netherlands). We incubated the tubes at constant temperature of $20^{\circ} \mathrm{C}$ in the dark using a climate-controlled chamber (Economic Lux chamber, Snijders Labs, Tilburg, the Netherlands).

After incubation, we collected $6.2 \mathrm{ml}$ of headspace air from each tube using a syringe and stored it into pre-evacuated $5.9 \mathrm{~mL}$ Exetainer vial (Labco Ltd., Buckinghamshire, UK). Samples were collected after $4 \mathrm{~h}$ of incubation for labile substrates (glucose, sucrose, glycine, oxalic acid, citric acid and yeast) and after $24 \mathrm{~h}$ for more recalcitrant substrates (lignin, cellulose).

The concentration of $\mathrm{CO}_{2}$ in the gas vials (over pressure of 1 bar) was measured by injecting $250 \mu \mathrm{l}$ of each sample in a Trace Ultra GC gas chromatograph equipped with a flame ionization detector with methanizer (mFID) (Interscience BV, Breda, the Netherlands) and a TriplusRSH auto-sampler (Interscience BV, Breda, the Netherlands), and a Rt-QBOND (30 m, 0.32 mm ID) capillary column (Restek, Bellefonte USA). We used helium 5.0 as a carrier gas, a sample split ratio of $1: 20$ and set oven temperature at $50{ }^{\circ} \mathrm{C}$ with a flow of $5 \mathrm{ml}$. We used a calibration curve of known concentrations of $\mathrm{CO}_{2}$ ranging from 0 to $4600 \mathrm{ppm}$ of $\mathrm{CO}_{2}$ preprared out of a reference gas $(2.38 \% \mathrm{CO} 2$ in synthetic air, 
Westfalen AG, Munster, Germany) to determine the amount of $\mathrm{CO}_{2}$ in our samples. Chromeleon 7.2 Data System Software (Thermo Scientific Waltham, USA) was used to automatize the measurements and process data.

Extracellular enzyme activity

Remaining rhizosphere soil was kept at $-20^{\circ} \mathrm{C}$ for further analyses of extracellular enzyme activity in the soil. We measured soil enzyme activity using high-throughput fluorometric measurements, where a gain of fluorescence over the incubation time represents the amount of enzymatic activity (Baldrian 2009). We determined the potential activity of 3 enzymes in soils involved in different pathways of carbon and nutrient cycling: glucosidase, phosphatase and aminopeptidase. Enzyme activity was measured in the 80 soil samples of the last time point (12 weeks). Briefly, $1 \mathrm{~g}$ of fresh soil was weighed into a clean glass jar before adding $50 \mathrm{ml}$ of sodium acetate buffer $(2.5 \mathrm{mM}, \mathrm{pH}=5.5)$. Vials were then capped tightly and shaken in a horizontal shaker for $10 \mathrm{~min}$ at $330 \mathrm{rpm}$ in order to obtain the soil homogenate. Fluorogenic substrates 4-methylumbellyferyl- $\beta$-Dglucopyranoside (MUFG), 4-methylumbellyferyl-phosphate (MUFP) and L-alanine-7amido-4-methylcoumarin (AMCA) were purchased (Sigma-Aldrich Chemie NV, Zwijndrecht, Netherlands). We dissolved all substrates in DMSO at concentration of 2.5 $\mathrm{mM}$ for AMCA and $2.75 \mathrm{mM}$ for MUFG and MUFP. A 40ul of substrate solution was mixed with $250 \mu \mathrm{l}$ of soil homogenate in each well of a black 96-well plate. Three technical replicates were included per soil sample and enzyme activity. We calibrated concentrations of enzyme activity product by a dilution curve made from a stable form of the fluorogenic compounds (1.0 mM methylumbellyferol (MUF) and $1.0 \mathrm{mM}$ 7aminomethyl-4-coumarin (AMC) (Sigma-Aldrich Chemie NV, Zwijndrecht, Netherlands). Fluorescence was measured at time $0 \mathrm{~h}$ and after $2 \mathrm{~h}$ of incubation at $40{ }^{\circ} \mathrm{C}$. We used a 96well plate reader with an excitation and emission wavelengths of $360 \mathrm{~nm}$ and $460 \mathrm{~nm}$, respectively (Synergy HT, BioTek Instruments, Winooski, Vermont, USA). We compared the measured fluorescence in our samples, after subtraction of the blank, with standard curves of MUF and AMC to calculate the amount of enzymatic product formed over the incubation time. A unit of enzyme activity is defined as the amount of enzyme reaction product ( $\mu \mathrm{mol})$ per gram of dry soil and hour. 
Table S3.1. List of plant genera and species used in the experiment and the coordinates of the field locations where soils were sampled

\begin{tabular}{|c|c|c|c|c|}
\hline Genera & Species & Origin & Site & Coordinates \\
\hline \multirow[t]{10}{*}{ Centaurea } & jacea & Native & 1 & $\mathrm{~N} 51^{\circ} 52.076^{\prime} \mathrm{E} 5^{\circ} 59.529^{\prime}$ \\
\hline & & & 2 & $\mathrm{~N} 51^{\circ} 52.764^{\prime} \mathrm{E}^{\circ} 00.292^{\prime}$ \\
\hline & & & 3 & $\mathrm{~N} 51^{\circ} 52.985^{\prime} \mathrm{E} 5^{\circ} 42.911^{\prime}$ \\
\hline & & & 4 & $\mathrm{~N} 51^{\circ} 52.787^{\prime} \mathrm{E} 5^{\circ} 43.751^{\prime}$ \\
\hline & & & 5 & $\mathrm{~N} 51^{\circ} 52.036^{\prime} \mathrm{E} 6^{\circ} 01.505^{\prime}$ \\
\hline & stoebe * & Range-expander & 1 & $\mathrm{~N} 51^{\circ} 51.599^{\prime} \mathrm{E} 5^{\circ} 53.332^{\prime}$ \\
\hline & & & 2 & $\mathrm{~N} 51^{\circ} 51.605^{\prime} \mathrm{E}^{\circ} 53.332^{\prime}$ \\
\hline & & & 3 & $\mathrm{~N} 51^{\circ} 51.606^{\prime} \mathrm{E} 5^{\circ} 53.342^{\prime}$ \\
\hline & & & 4 & $\mathrm{~N} 51^{\circ} 51.605^{\prime} \mathrm{E} 5^{\circ} 53.354^{\prime}$ \\
\hline & & & 5 & $\mathrm{~N} 51^{\circ} 51.609^{\prime} \mathrm{E} 5^{\circ} 53.350^{\prime}$ \\
\hline \multirow[t]{10}{*}{ Geranium } & molle & Native & 1 & $\mathrm{~N} 51^{\circ} 46.700^{\prime} \mathrm{E} 5^{\circ} 55.612^{\prime}$ \\
\hline & & & 2 & $\mathrm{~N} 51^{\circ} 52.006^{\prime} \mathrm{E} 5^{\circ} 59.429^{\prime}$ \\
\hline & & & 3 & $\mathrm{~N} 51^{\circ} 52.743^{\prime} \mathrm{E}^{\circ} 00.267^{\prime}$ \\
\hline & & & 4 & $\mathrm{~N} 51^{\circ} 52.627^{\prime} \mathrm{E} 6^{\circ} 00.032^{\prime}$ \\
\hline & & & 5 & $\mathrm{~N} 51^{\circ} 52.641^{\prime} \mathrm{E} 6^{\circ} 00.120^{\prime}$ \\
\hline & pyrenaicum & Range-expander & 1 & $\mathrm{~N} 51^{\circ} 57.872^{\prime} \mathrm{E} 5^{\circ} 40.861^{\prime}$ \\
\hline & & & 2 & $\mathrm{~N} 51^{\circ} 46.702^{\prime} \mathrm{E} 5^{\circ} 55.579^{\prime}$ \\
\hline & & & 3 & $\mathrm{~N} 51^{\circ} 52.662^{\prime} \mathrm{E}^{\circ} 01.296^{\prime}$ \\
\hline & & & 4 & $\mathrm{~N} 51^{\circ} 51.856^{\prime} \mathrm{E}^{\circ} 00.938^{\prime}$ \\
\hline & & & 5 & $\mathrm{~N} 51^{\circ} 51.822^{\prime} \mathrm{E} 6^{\circ} 00.953^{\prime}$ \\
\hline \multirow[t]{10}{*}{ Tragopogon } & pratensis & Native & 1 & $\mathrm{~N} 51^{\circ} 52.972^{\prime} \mathrm{E} 5^{\circ} 42.850^{\prime}$ \\
\hline & & & 2 & $\mathrm{~N} 51^{\circ} 52.869^{\prime} \mathrm{E}^{\circ} 43.318^{\prime}$ \\
\hline & & & 3 & $\mathrm{~N} 51^{\circ} 52.797^{\prime} \mathrm{E} 5^{\circ} 43.466^{\prime}$ \\
\hline & & & 4 & $\mathrm{~N} 51^{\circ} 52.781^{\prime} \mathrm{E} 5^{\circ} 43.731^{\prime}$ \\
\hline & & & 5 & $\mathrm{~N} 51^{\circ} 52.468^{\prime} \mathrm{E} 5^{\circ} 46.793^{\prime}$ \\
\hline & dubius * & Range-expander & 1 & $\mathrm{~N} 51^{\circ} 50.161^{\prime} \mathrm{E} 5^{\circ} 51.224^{\prime}$ \\
\hline & & & 2 & $\mathrm{~N} 51^{\circ} 50.152^{\prime} \mathrm{E} 5^{\circ} 51.228^{\prime}$ \\
\hline & & & 3 & $\mathrm{~N} 51^{\circ} 50.285^{\prime} \mathrm{E} 5^{\circ} 51.113^{\prime}$ \\
\hline & & & 4 & $\mathrm{~N} 51^{\circ} 50.248^{\prime} \mathrm{E} 5^{\circ} 51.075^{\prime}$ \\
\hline & & & 5 & $\mathrm{~N} 51^{\circ} 50.253^{\prime} \mathrm{E} 5^{\circ} 51.072^{\prime}$ \\
\hline \multirow[t]{10}{*}{ Rorippa } & sylvestris & Native & 1 & $\mathrm{~N} 51^{\circ} 52.275^{\prime} \mathrm{E} 5^{\circ} 54.398^{\prime}$ \\
\hline & & & 2 & $\mathrm{~N} 51^{\circ} 52.104^{\prime} \mathrm{E} 5^{\circ} 56.275^{\prime}$ \\
\hline & & & 3 & $\mathrm{~N} 51^{\circ} 52.145^{\prime} \mathrm{E} 5^{\circ} 59.389^{\prime}$ \\
\hline & & & 4 & $\mathrm{~N} 51^{\circ} 52.652^{\prime} \mathrm{E}^{\circ} 00.010^{\prime}$ \\
\hline & & & 5 & $\mathrm{~N} 51^{\circ} 52.761^{\prime} \mathrm{E} 6^{\circ} 00.255^{\prime}$ \\
\hline & austriaca & Range-expander & 1 & $\mathrm{~N} 51^{\circ} 52.316^{\prime} \mathrm{E} 5^{\circ} 54.397^{\prime}$ \\
\hline & & & 2 & $\mathrm{~N} 51^{\circ} 52.177^{\prime} \mathrm{E} 5^{\circ} 56.197^{\prime}$ \\
\hline & & & 3 & $\mathrm{~N} 51^{\circ} 52.057^{\prime} \mathrm{E} 5^{\circ} 59.462^{\prime}$ \\
\hline & & & 4 & $\mathrm{~N} 51^{\circ} 52.204^{\prime} \mathrm{E} 5^{\circ} 59.468^{\prime}$ \\
\hline & & & 5 & $\mathrm{~N} 51^{\circ} 52.239^{\prime} \mathrm{E} 5^{\circ} 59.495^{\prime}$ \\
\hline
\end{tabular}

(*) Range-expanders Centaurea stoebe and Tragopogon dubius are rare in the Netherlands and therefore,

field soils of these species were collected from 5 individuals that were maximally $100 \mathrm{~m}$ apart 
Table S3.2. PERMANOVA test on Bray-Curtis dissimilarity matrix of bacterial communities in the field inocula soils (999 permutations)

\begin{tabular}{lll}
\hline Factor & $\mathbf{R}^{2}$ & Signif. \\
\hline Soil $\mathrm{pH}$ & 0.188 & $* * *$ \\
Soil $\left(\mathrm{NO}_{3}-\mathrm{NO}_{2}\right)-\mathrm{N}$ & 0.059 & $* *$ \\
Soil $\mathrm{NH}_{4}-\mathrm{N}$ & 0.061 & $* *$ \\
Soil $\mathrm{PO}_{4}$ & 0.029 & $*$ \\
Soil $\mathrm{CN}$ ratio & 0.065 & $* *$ \\
Plant species & 0.216 & $* *$ \\
\hline Significance levels: $* \mathrm{p}<0.05 ; * * \mathrm{p}<0.01 ; * * * \mathrm{p}<0.001$
\end{tabular}

Table S3.3. Effects of plant genus and plant origin and their interaction on soil abiotic parameters and bacterial community characteristics of the field soils were analyzed using 2-way ANOVA

\begin{tabular}{|c|c|c|c|c|c|c|}
\hline \multirow[b]{2}{*}{ Soil parameters } & \multicolumn{2}{|c|}{ Plant genus } & \multicolumn{2}{|c|}{ Plant origin } & \multicolumn{2}{|c|}{ Genus * Origin } \\
\hline & $\mathrm{F}$ & Signif. & $\mathrm{F}$ & Signif. & $\mathrm{F}$ & Signif. \\
\hline $\mathrm{pH}$ & 33.461 & $* * *$ & 2.495 & ns & 2.641 & . \\
\hline Nitrate $\left(\left(\mathrm{NO}_{3}^{-}-\mathrm{NO}_{2}^{-}\right)-\mathrm{N}\right)$ & 6.705 & $* *$ & 5.344 & $*$ & 1.667 & ns \\
\hline Ammonia $\left(\mathrm{NH}_{4}{ }^{+}-\mathrm{N}\right)$ & 19.521 & $* * *$ & 4.705 & $*$ & 4.932 & $* *$ \\
\hline P-Olsen & 6.196 & $* *$ & 0.715 & ns & 1.654 & ns \\
\hline $\mathrm{C}: \mathrm{N}$ ratio & 18.768 & $* * *$ & 4.048 & . & 8.203 & $* * *$ \\
\hline Bacterial community & $\mathrm{F}$ & Signif. & $\mathrm{F}$ & Signif. & $\mathrm{F}$ & Signif. \\
\hline OTU richness & 12.817 & $* * *$ & 0.811 & ns & 0.756 & ns \\
\hline Diversity (H') & 11.129 & $* * *$ & 2.249 & ns & 1.089 & $\mathrm{~ns}$ \\
\hline Evenness & 3.574 & * & 0.981 & ns & 1.259 & $\mathrm{~ns}$ \\
\hline
\end{tabular}

Significance levels: $n s \mathrm{p}>0.1 ; \mathrm{p}<0.1 ; * \mathrm{p}<0.05 ; * * \mathrm{p}<0.01 ; * * * \mathrm{p}<0.001 ; * * * * \mathrm{p}<0.0001$ 

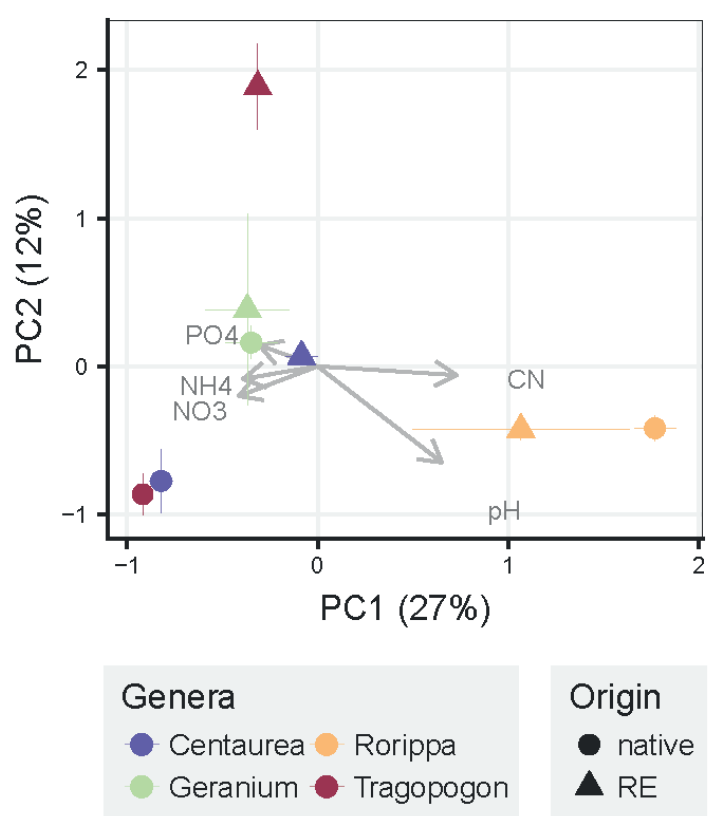

Fig. S3.1. Principal Coordinate Analyses of the bacterial community of field soils, which originate from locations where the plant species grown in the field and served as inocula in our experiment. Soil abiotic properties are projected in the ordination plot as supplementary variables (arrows). Symbols are means $\pm \mathrm{SE}(\mathrm{N}=5)$. Different colours represent the different plant pairs of range expander and congeneric natives. Within each pair, circles represent the native plant species and triangles the range-expander. 

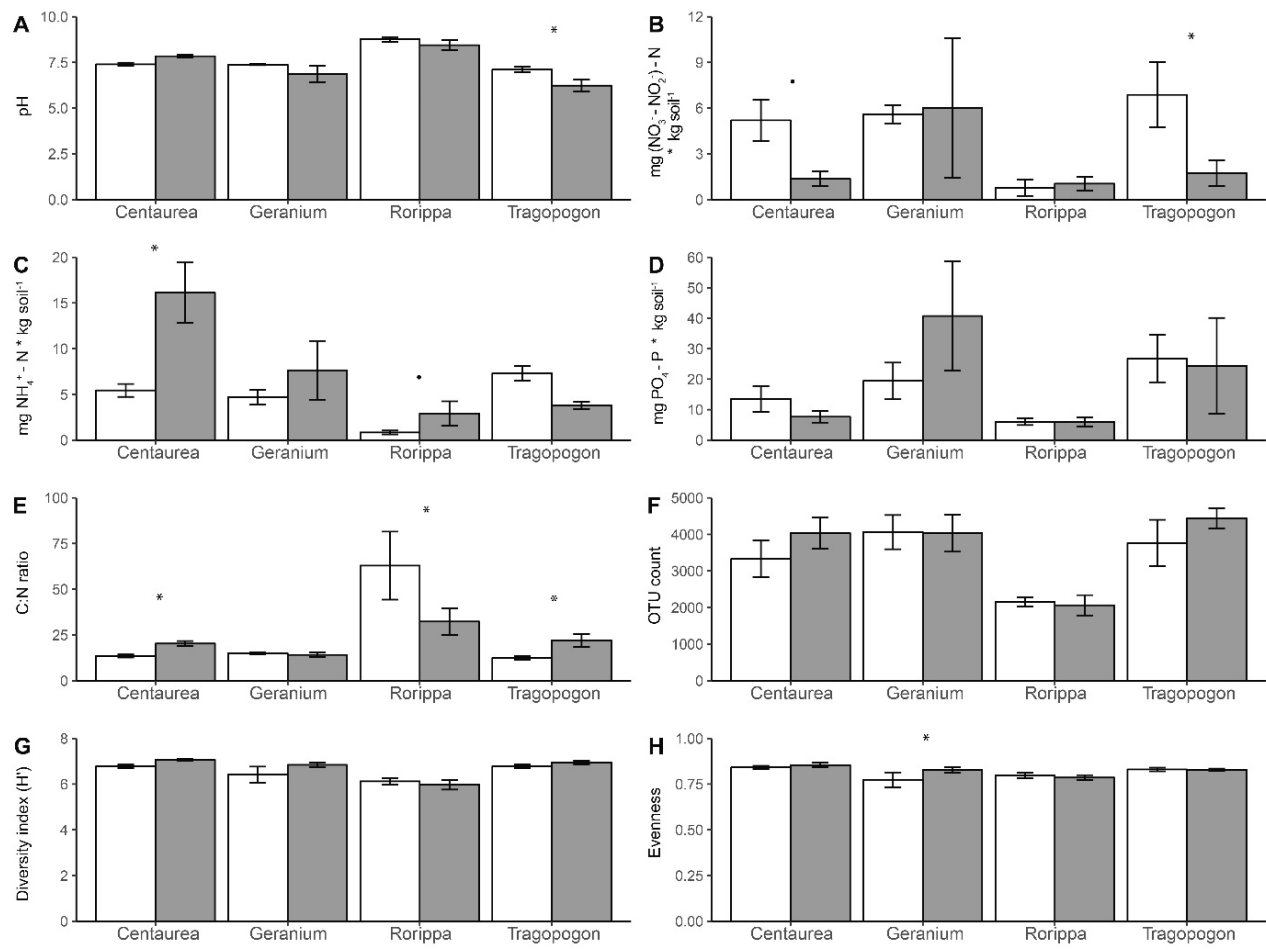

Fig. S3.2. Soil properties of the soils collected from field populations, and used as inocula in the experiment. White bars represent native plant species and grey bars represent range-expanding plant species in each plant genus. (A) $\mathrm{pH}$, (B) nitrate and nitrite availability, (C) ammonium availability, (D) phosphate availability, (E) soil C: N ratio, (F) 16S OTU richness, (G) OTU Shannon diversity index, $\mathrm{H}^{\prime}$ (1) and $(\mathbf{H})$ community evenness, $\mathrm{EH}^{(2)}$. Means with standard error $(\mathrm{n}=5)$ are presented for native (white bars) and range-expanding (grey bars) plant species. Asterisk $\left(^{*}\right)$ and $\operatorname{dot}($.$) symbols indicate \mathrm{P}<0.05$ and $\mathrm{P}<0.1$, respectively, in pairwise within-genus comparisons. (1) Shannon diversity index $H^{\prime}=$ $-\sum_{i=1}^{n} p_{i} \ln p_{i}$ where $p i$ is the proportion of species $i$ relative to the total number of species. (2) Community evenness $E_{H}=H / \ln S$ where $S$ is the total number of OTUs. 

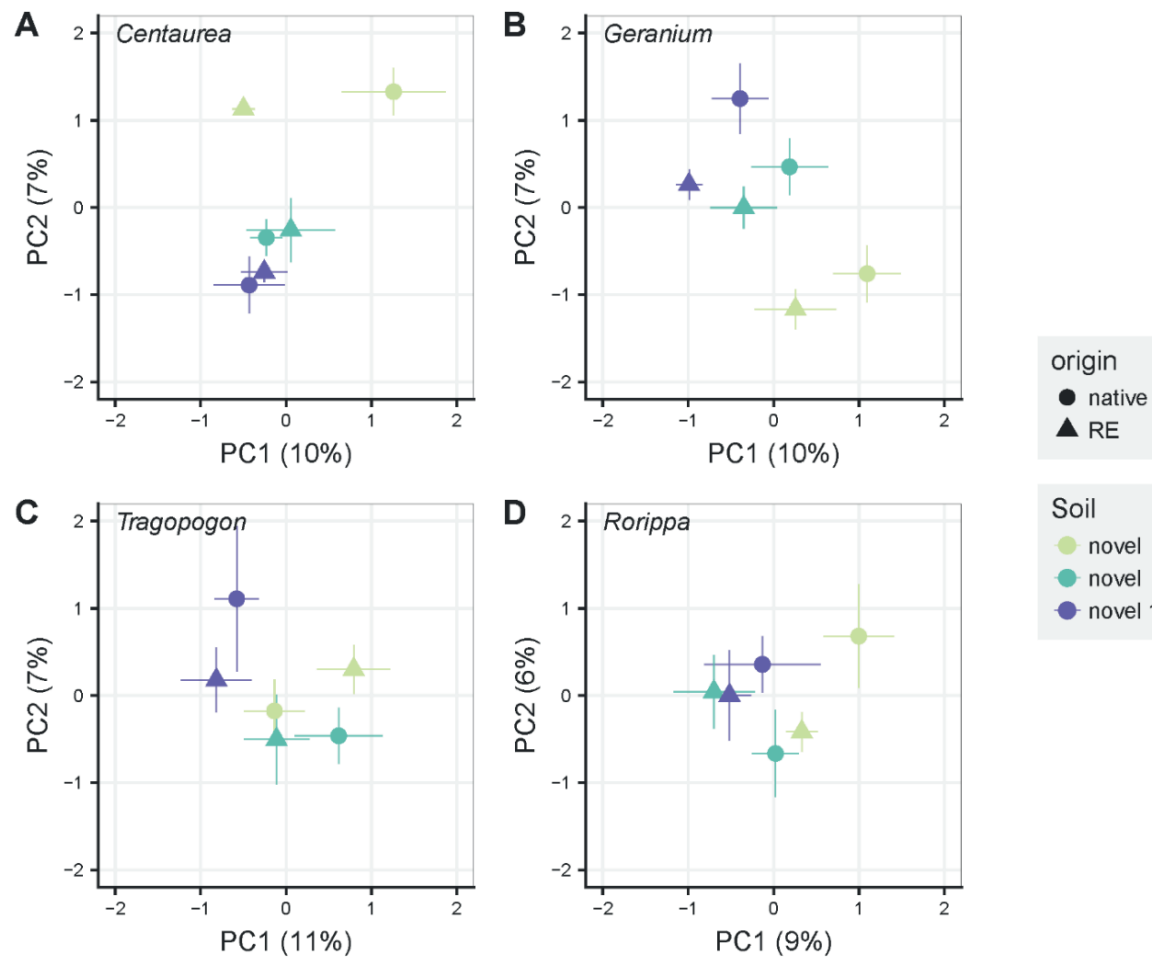

Soil

novel 4

- novel 8

novel 12

Fig. S3.3. Principal Coordinate Analyses of the rhizosphere bacterial community composition for each pair of a range-expander and its congeneric native plant species (A: Centaurea, B: Geranium, C: Tragopogon, D: Rorippa) grown in "novel" soils. The symbols are means $\pm \mathrm{SE}(\mathrm{N}=5)$. Within each pair, circles represent the native plant species and triangles the range-expander. Colors indicate time of harvest (4, 8 and 12 weeks) as noted in the legend. 

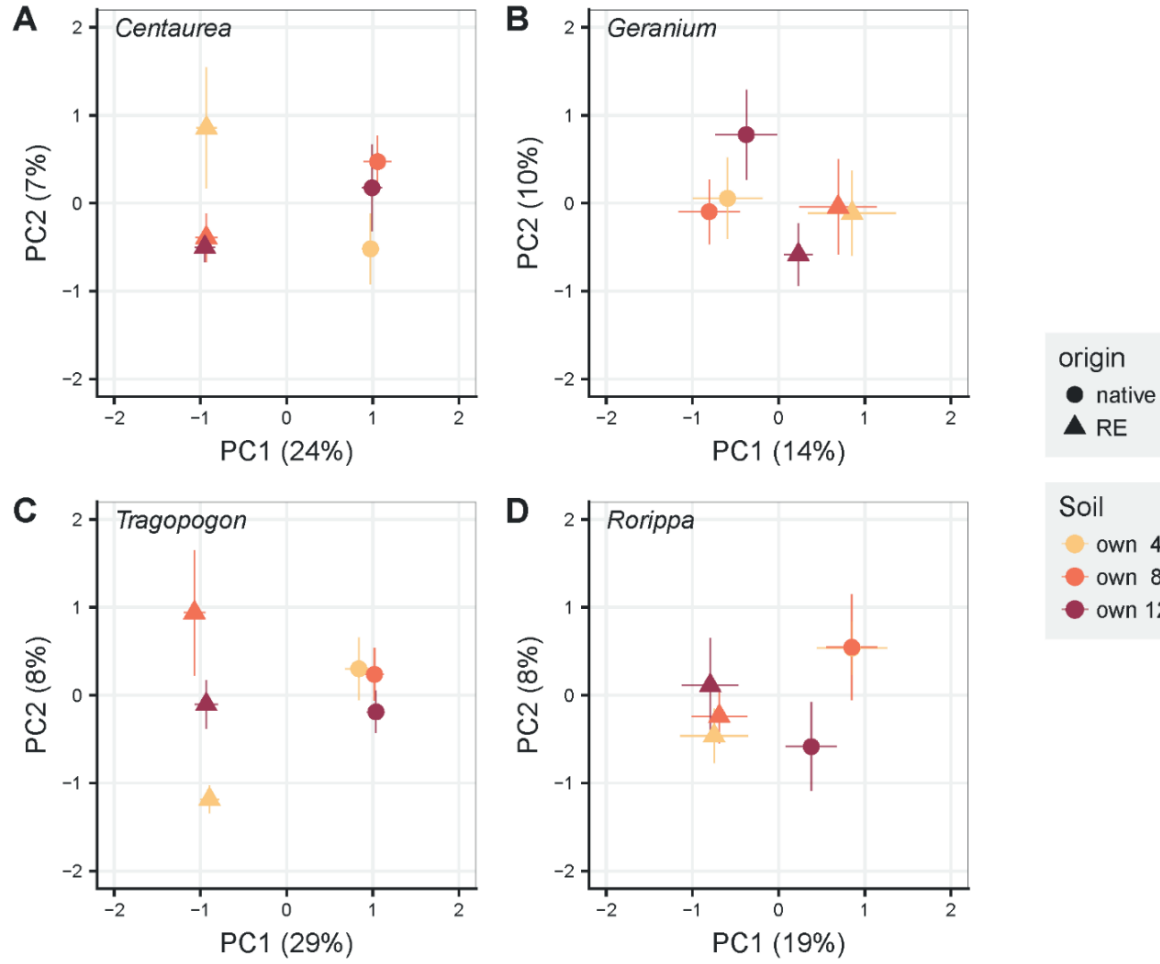

Soil

own 4

own 8

own 12

Fig. S3.4. Principal Coordinate Analyses of the rhizosphere bacterial community composition for each pair of a range-expander and its congeneric native plant species (A: Centaurea, B: Geranium, C: Tragopogon, D: Rorippa) grown in "own" soils. The symbols are means $\pm \mathrm{SE}(\mathrm{N}=5)$. Within each pair, circles represent the native plant species and triangles the range-expander. Colors indicate time of harvest ( 4,8 and 12 weeks) as noted in the legend. 

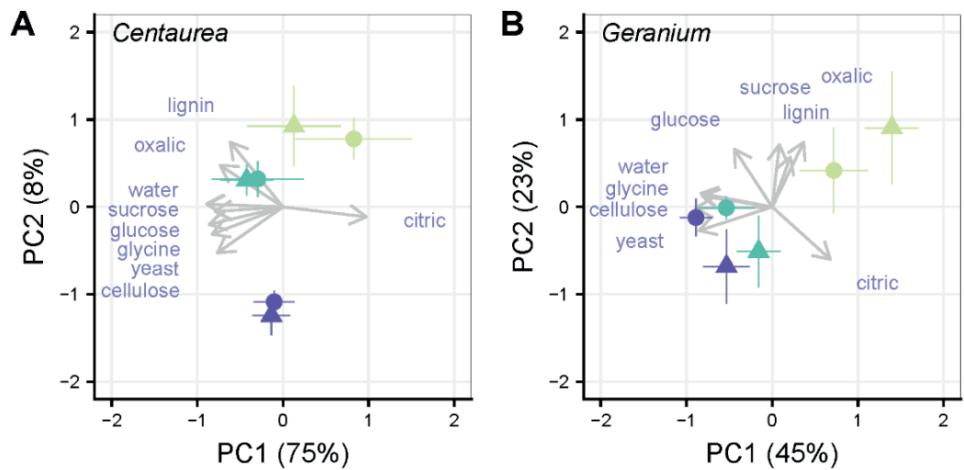
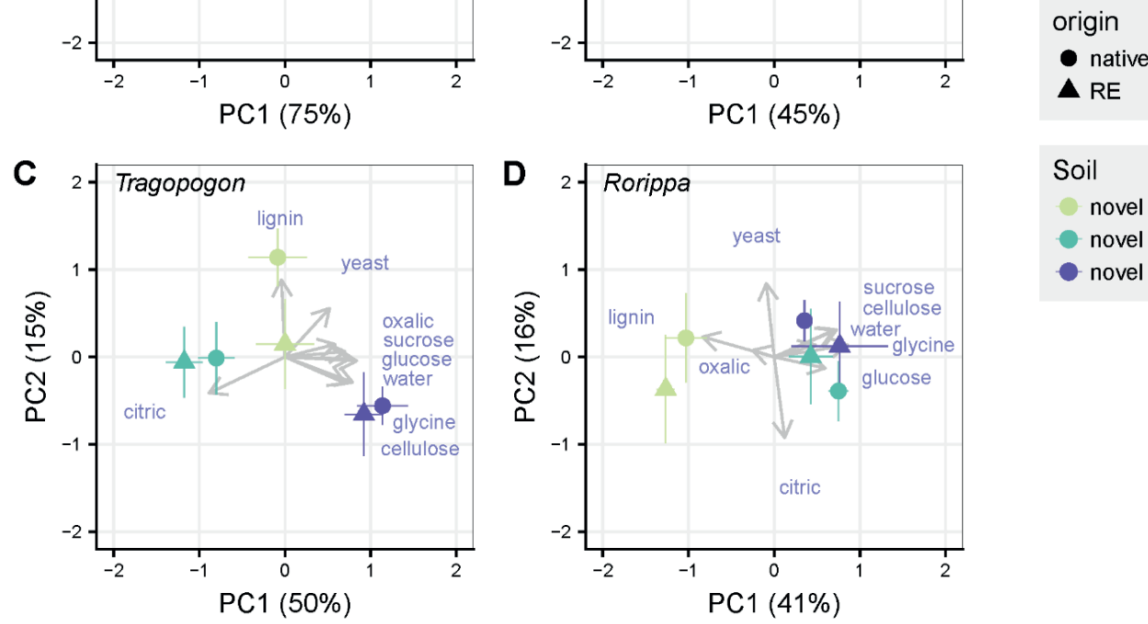

Soil

novel 4

- novel 8

novel 12

Fig. S3.5. Principal Coordinate Analyses of the catabolic response profiles for each pair of a rangeexpander and its congeneric native plant species (A: Centaurea, B: Geranium, C: Tragopogon, D: Rorippa) grown in "novel" soils. The symbols are means $\pm \mathrm{SE}(\mathrm{N}=5)$. Within each pair, circles represent the native plant species and triangles the range-expander. Arrows representing each substrate are displayed over the ordination plot as supplementary variables. Colors indicate time of harvest $(4,8$ and 12 weeks) as noted in the legend. 

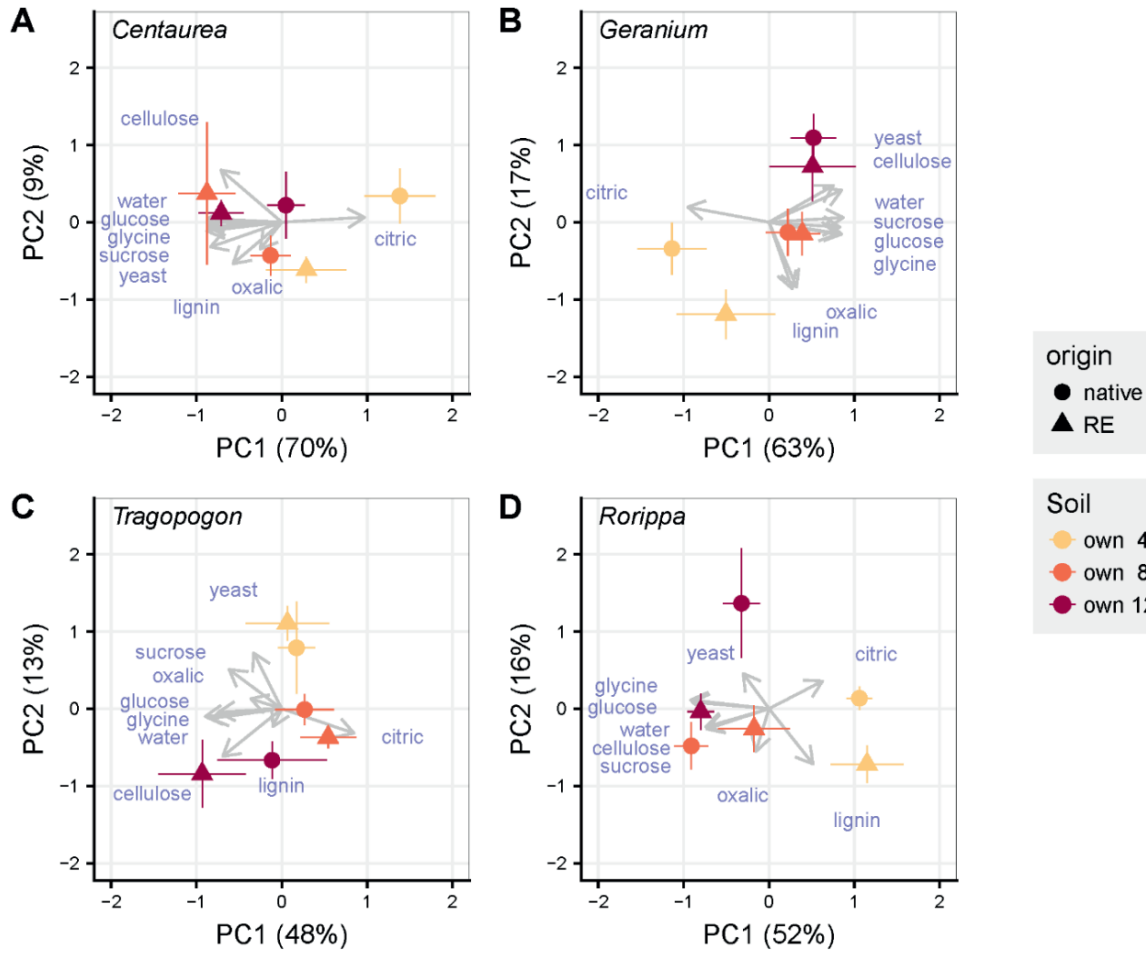

Soil

own 4

- own 8

own 12

Fig. S3.6. Principal Coordinate Analyses of the catabolic response profiles for each pair of a rangeexpander and its congeneric native plant species (A: Centaurea, B: Geranium, C: Tragopogon, D: Rorippa) grown in "own" soils. The symbols are means $\pm \mathrm{SE}(\mathrm{N}=5)$. Within each pair, circles represent the native plant species and triangles the range-expander. Arrows representing each substrate are displayed over the ordination plot as supplementary variables. Colors indicate time of harvest $(4,8$ and 12 weeks) as noted in the legend. 


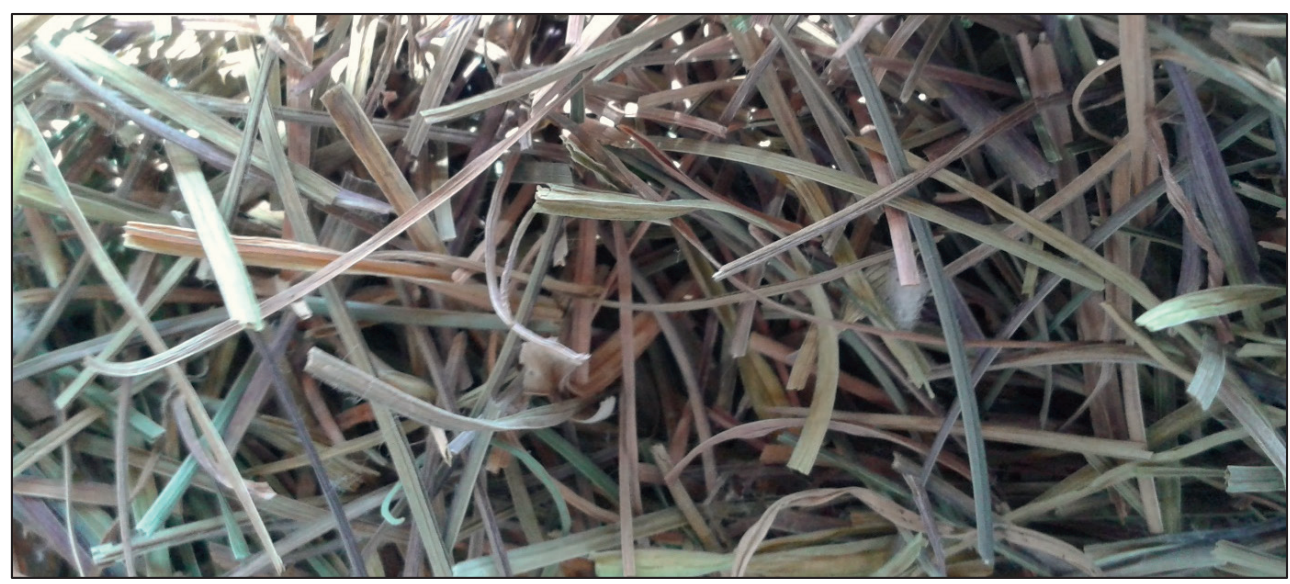




\section{Chapter 4}

\section{Rhizosphere and litter feedbacks to range- expanding plant species and related natives}

Marta Manrubia, Wim H. van der Putten, Carolin Weser \& Ciska Veen

Manuscript in review 


\section{Abstract}

Plant-soil feedback (PSF) results from the legacy that plants leave in the composition of soil communities and abiotic soil properties. PSF is known to be the net outcome of plantsoil interactions induced by the rhizosphere and by litter inputs into the soil, however, we have little understanding of their individual contributions. Here, we examine feedback effects from the rhizosphere of living plants, decomposing litter, and their combination. We used climate warming-induced range-expanding plant species, to assess how PSF may play a role in their establishment and performance in the new range. We tested PSF effects on plant biomass production, as well as on decomposition. We tested the hypothesis that the plant rhizosphere provides less negative feedback to range expanders than to natives, and that decomposition does not provide such an advantage because these soil biota are expected to be less specialized. We used a highly sensitive PSF approach by using soil from the congener species as an "away" soil control, as that would indicate whether rangeexpanders may have lost their specialized soil biota upon arrival in the novel range soil.

Our results show that although range-expanding plant species and their congeneric natives developed neutral PSF in both rhizosphere and litter-conditioned soils, two out of four range-expanders produced significantly more biomass than natives in soils conditioned by litter. Shoot litter from two out of four range-expanding species showed more decomposition than natives, but decomposition was unaffected by soil conditioning. In general, biomass of plants growing in soils conditioned by plant rhizosphere plus litter may be predicted from biomass production in soils conditioned by living plant rhizosphere or plant litter alone. In our experimental set-up, using control soils that are known to produce conservative PSF values, PSF effects via the rhizosphere and litter were not affected by plant origin. Still, range-expanders appeared to benefit more from high nutrient availability in the soil than natives, which will provide an advantage under otherwise equal environmental conditions. 


\section{Introduction}

Growing plants induce changes in the composition of soil communities and the physicochemical soil environment (Berg and Smalla 2009). The legacy of these changes can alter the growth and fitness of themselves, their neighbours and subsequent plants via socalled plant-soil feedback (PSF) effects (Bever et al. 1997, Kulmatiski et al. 2008). The biotic component of PSF is driven by three main groups of soil biota: enemies (including pathogens and herbivores), mutualistic symbionts (such as mycorrhizal fungi and rhizobia), and decomposers, which are responsible for the breakdown of organic compounds (van der Putten et al. 2016). The mechanism by which these different soil biota can steer plant performance is a net effect of direct interactions with living plant roots, mostly pathogens and mutualistic symbionts, and indirect interactions with plants via the breakdown of plant litter, mostly by decomposer organisms, or by changes in the abiotic soil environment (Wardle et al. 2004). Little is known about the relative contribution of these direct and indirect interactions to overall net PSF effects and subsequent plant growth responses, whereas this knowledge is relevant in order to understand how global environmental changes may influence spatio-temporal dynamics in plant communities (van der Putten et al. 2013, van der Putten et al. 2016).

The rhizosphere harbors a large diversity of soil microorganisms and invertebrates (Philippot et al. 2013), and plant species affect rhizosphere communities in a speciesspecific way (Berg and Smalla 2009). Rhizosphere organisms include specific plant pathogens, plant growth-promoting bacteria or mutualistic symbionts, which can have direct beneficial, neutral or harmful effects on the host plant (Singh et al. 2004, Raaijmakers et al. 2009, Mendes et al. 2013). They use living plant roots or root exudates released by plants, both serving as carbon and nutrient sources and, in turn, affect plant performance by feeding damage but also by regulating water and nutrient availability and the production of volatile organic compounds (Bailly and Weisskopf 2012, Mendes et al. 2013). In this way plants leave behind a legacy effect in the soil that may affect subsequent plant growth. Many previous studies have focused on rhizosphere-induced PSF effects and showed that these can range from negative (pathogens and parasites dominate) to positive (mutualistic symbionts dominate) (Kulmatiski et al. 2008, Ma et al. 2017, Lekberg et al. 2018).

Plant-soil feedback effects can also be affected by plant litter decomposition (Ehrenfeld et al. 2005, Eppinga et al. 2011, Eppinga and Molofsky 2013, Zhang et al. 2016b). Besides 
shaping a specific rhizosphere community of soil biota, plant species may also develop distinct and specific decomposer communities (Keiser et al. 2011, Elgersma et al. 2012) via the quantity, quality (i.e. chemical properties) and timing of litter inputs into the soil (Austin et al. 2014). As a result, decomposing plant litter may leave a plant species-specific legacy in the form of altered nutrient status, secondary chemistry, water availability and altered physical soil properties (Ehrenfeld 2010, Freschet et al. 2013). These alterations will indirectly, and sometimes even directly determine subsequent plant performance and therefore contribute to PSF effects. In addition, locally specialized decomposer communities may accelerate decomposition rates, referred to as home-field advantage (Gholz et al. 2000, Ayres et al. 2009b), which may also influence subsequent plant growth by PSF. Even though litter-mediated effects on soil community composition, functioning and the resulting PSF effects are expected to be less specific than those mediated via the rhizosphere (Ke et al. 2015), we still have poor understanding of the relative importance of the decomposer contribution to net PSF. For example, the decomposer pathway may reduce negative effects mediated via the rhizosphere pathway (Zhang et al. 2016b). Therefore, in order to further understand how PSF impacts on plant growth and performance, it will be important to take both effects mediated by rhizosphere and litter decomposition into account.

PSF effects have been proposed to play an important role during the establishment of introduced exotic plant species (Klironomos 2002, Blumenthal et al. 2009, Eppinga et al. 2011). PSF is often less negative, or more positive in novel than in native ranges (Reinhart et al. 2003, Callaway et al. 2004) and soil biota in the new range may alter plant resource allocation strategies (te Beest et al. 2009). Thus far, most PSF studies on introduced plants have considered exotic species that have been introduced from other continents (Suding et al. 2013). However, many plant species are also migrating intra-continentally, because ongoing climate warming enables range expansion to higher latitudes and altitudes (Parmesan and Yohe 2003, Chen et al. 2011, Alexander et al. 2015). During these intracontinental range shifts, PSF effects may vary between the new and original range, because of different dispersal rates of plants and soil biota (Berg et al. 2010) and different specificities of soil biota involved in rhizosphere and litter-induced feedback interactions (van der Putten 2012).

Range-expanding plant species have been shown to benefit from escaping their natural enemies in the rhizosphere, thereby experiencing less negative, or even positive PSF in the 
new compared to the original range (Van Grunsven et al. 2010, Dostálek et al. 2016). In addition, range expanders may increase soil nitrogen availability (Meisner et al. 2012) and may have different nutrient acquisition strategies or time of peak growth than natives (Mariotte et al. 2017). However, it is largely unknown how indirect interactions, such as through plant litter decomposition, may contribute to net PSF during plant range expansion. In order to further understand how direct effects from pathogens and mutualistic symbionts and indirect effects from decomposer organisms may contribute to PSF, a first step is to consider feedback effects from rhizosphere and litter both separately and in combination. We will do this by comparing range expanders in their new range with co-occurring congeneric native species, as these range expanders and natives are expected to differ in the degree to which direct and indirect PSF components contribute to net PSF effects (van der Putten 2012).

We designed a two-phase feedback experiment in the greenhouse in order to determine PSF effects of range-expanding plant species and congeneric related species that are native in the new range. Eight plant species (four range expanders and four natives) were grown in soils conditioned by living plants and/or plant litter. We tested four hypotheses: (1) For rhizosphere feedback effects, range expanders do not show different growth responses to soils conditioned by either conspecifics or congeneric natives, whereas natives will perform worst in their own soil. (2) For litter feedback effects, both range-expanders and congeneric natives will perform best in soils conditioned by litter of range-expanders, as they are expected to have higher quality litter and decompose more than litter of natives (Meisner et al. 2012). (3) Because litter feedback effects are expected to alleviate nutrient limitations in plant growth potential, plant growth responses in the combined treatment of rhizosphere and plant litter conditioning should be predictable from responses to individual rhizosphere and litter conditioning. (4) Litter breakdown is greater for litter of native plant species in their own conditioned soils, but not for range-expanders due to the lack of specialized decomposers in the new range.

In order to test the hypotheses, we have conditioned soils by growing plants, incorporating plant litter in the soil, or a combination of growing plants and litter incorporation. We conditioned the soils using living plants or litter from four rangeexpanding and four congeneric related native plant species, all individually. At the end of the conditioning phase we have assessed the functional capacity of the soil community, soil nutrient status, plant growth, and decomposition responses to the soil conditioning 
treatments, by growing plants and by decomposing litter in soils conditioned by conspecific and congeneric species. In our experiment, the control for PSF effects was based on a comparison of performance in conspecific conditioned soil to that in congeneric conditioned soil, which enabled us to detect eventual high degree intra-genus specialization of the soil communities between natives and range expanders. This method is far more sensitive than using a sterilized or a more general "away" soil as a control, so that we would not be able to detect whether range expanders in general are having less negative PSF than congeneric natives, as done in other studies on plant range shifts (Engelkes et al. 2008, Van Grunsven et al. 2010, Dostálek et al. 2016). Nevertheless, this approach allowed us to tease apart PSF effects of living plant roots, decomposing litter, or both.

\section{Material and Methods}

\section{Plant species selection}

We used four pairs of range-expanding plant species and congeneric natives (Centaurea stoebe and Centaurea jacea, Geranium pyrenaicum and Geranium molle, Tragopogon dubius and Tragopogon pratensis, Rorippa austriaca and Rorippa sylvestris, respectively). Seeds were collected from field populations or purchased from a commercial supplier (Cruydt Hoeck, Nijeberkoop, the Netherlands) that harvests seeds from plant material originally collected from the field. All seeds were surface sterilized (3 $\mathrm{min}, 10 \%$ bleach solution) and germinated on sterilized glass beads under controlled conditions $\left(20 / 10{ }^{\circ} \mathrm{C}\right.$ day/night, 16 $\mathrm{h}$ photoperiod). Due to their small size, Rorippa seeds were germinated directly in gammasterilized soil under the same controlled conditions (minimally 25 KGray, Syngenta BV, Ede, the Netherlands).

\section{Soil collection}

Background soil was collected from a former agricultural field in a riparian area near Beneden-Leeuwen, the Netherlands (N51 53.952, E05 33.670) and sterilized by gamma irradiation (minimally 25 KGray, Syngenta BV, Ede, the Netherlands). Inocula soils were collected from natural field populations of each of the plant species used. We collected soil inocula from 5 independent populations of each plant species obtaining a total of 5 replicates $\times 2$ origins (i.e., native and range-expander) $\times 4$ plant species pairs $=40$ independent soil inocula (Table S4.1). Soil inocula of each of the five independent replicates consisted of equal amounts of soil from each of the eight plant species (dry w/w). 
So, the soil inoculum for replicate one contained equal amounts of soil of replicate one of all eight plant species, the same for replicate two, three, four and five. In this way, we created five independent soil replicates, each consisting of a mix of field soil communities from all eight plant species.

\section{Experimental design}

The experiment consisted of two phases: a conditioning phase and a feedback phase. In the conditioning phase, we used three different types of conditioning treatments: with living plants (plant conditioning), with plant litter material (litter conditioning) and with both (plant + litter conditioning). This resulted into 120 pots $(8$ plant species $\times 3$ conditioning types of plant, litter, and both $\times 5$ replicates). After 13 weeks of conditioning, we harvested the plant material and used the soil in the feedback phase.

In the feedback phase we conducted two separate, simultaneously running experiments, one focusing on plant growth responses to soil conditioning and one focussing on litter decomposition responses to soil conditioning. For the feedback experiment on plant growth, we grew individual plants of each plant species in "own" soils (i.e. conditioned by themselves) and in "away" soils (i.e. conditioned by their congeneric species). There were 240 pots to test plant biomass production ( 3 conditioning type $\times 2$ own/away soils $\times$ 8 plant species $\times 5$ replicates). For the feedback experiment on litter decomposition, we used soils conditioned by plant litter material (litter conditioning) to determine the effect of species-specific conditioning on breakdown of shoot and root litter of the same plant species and the congeneric species. The feedback phase included 200 incubation microcosms $(2$ shoot/root litter $\times 2$ own/away soil $\times 8$ plant species $\times 5$ replicates +40 control soil only). Control soils were incubations containing only the 40 conditioned soils (8 plant species $\times 5$ replicates) and with no litter added, which allow to quantify and correct for the basal respiration of the soil.

\section{Conditioning phase}

We filled $3 \mathrm{~L}$ pots with sterilized soil mixed with $10 \%$ of live soil inocula (dry w/w). All soils were kept at $60 \%$ of the water holding capacity by watering to pre-determined weight twice a week. We incubated the pots with the soil in the greenhouse for 11 days to allow the soil inocula to establish before starting the conditioning by plants, litter, and plants + litter. We used litter of both shoots and roots, which was harvested from senescing greenhouse-grown plants. Root material was washed and both shoots and roots were air- 
dried and sterilized prior to use in the experiment (minimally 25 KGray, Syngenta BV, Ede, the Netherlands). We fragmented litter in pieces of appr. $1 \times 1 \mathrm{~cm}$ and added in total $6 \mathrm{~g}$ of dry litter per pot (50-50 \% shoot-root litter). The amount of litter was chosen to be representative of the average litter fall to the soil in temperate ecosystems (Peñuelas et al. 2007). Litter was mixed through the soil for each individual pot separately. Soils in the pots that received no litter were mixed in the same mechanical way to make all soils exposed to the same degree of disturbance. Subsequently, we planted three individuals of each plant species per pot and grew them for 13 weeks under controlled greenhouse conditions $\left(21 / 16{ }^{\circ} \mathrm{C}, 16 \mathrm{~h}\right.$ photoperiod supplied with $600 \mathrm{~W}$ high pressure sodium lamps at $250 \mu \mathrm{mol} \mathrm{m} \mathrm{m}^{-2} \mathrm{~s}^{-1}$ PAR and average air humidity of $60 \%$ ).

After 13 weeks of soil conditioning, we removed the plants from the soil, washed the roots, dried both above and belowground biomass at $40{ }^{\circ} \mathrm{C}$ for five days and measured biomass. We immediately took a subsample of soil from each pot to analyse the soil catabolic response profile at the day of harvest and followed the protocol of Fierer et al. (2012). We added eight different organic substrates of varying complexity. Labile substrates were glucose, sucrose, glycine, oxalic acid, citric acid and yeast, and recalcitrant substrates were chitin and cellulose) separately to $4 \mathrm{~g}$ of soil (dry weight equivalent). Soils were weighed into $50 \mathrm{~mL}$ centrifuge tubes modified with a rubber O-ring in the inner part of the lid to ensure air tightness, and equipped the lids with a butyl rubber to allow headspace air sampling with a syringe. We then added $8 \mathrm{~mL}$ of substrate solution with concentrations ranging from 15 to $100 \mathrm{mM}$ depending on the compound as used by Fierer et al. (2012). We capped the vials tightly, flushed the headspace with $\mathrm{O}_{2}$-free air during 2 min at 1 bar (Westfalen Gassen Nederland BV, Deventer, the Netherlands) and incubated the vials at $20^{\circ} \mathrm{C}$ and darkness using a controlled climate chamber (Economic Lux chamber, Snijders Labs, Tilburg, the Netherlands). We measured the net accumulation of $\mathrm{CO}_{2}$ over a period of $4 \mathrm{~h}$ for labile substrates or $24 \mathrm{~h}$ for recalcitrant substrates. A headspace sample of $6.2 \mathrm{ml}$ was collected from each tube using a syringe and stored it into pre-evacuated $5.9 \mathrm{~mL}$ Exetainer vial (Labco Ltd., Buckinghamshire, UK).

The concentration of $\mathrm{CO}_{2}$ in the gas vials (over pressure of 1 bar) was measured by injecting $250 \mu \mathrm{l}$ of each sample in a Trace Ultra GC gas chromatograph equipped with a flame ionization detector with methanizer (mFID) (Interscience BV, Breda, the Netherlands) and a TriplusRSH auto-sampler (Interscience BV, Breda, the Netherlands), and a Rt-QBOND (30 m, 0.32 mm ID) capillary column (Restek, Bellefonte USA). We used 
helium 5.0 as a carrier gas, a sample split ratio of $1: 20$ and set oven temperature at $50{ }^{\circ} \mathrm{C}$ with a flow of $5 \mathrm{ml}$. We used a calibration curve of known concentrations of $\mathrm{CO}_{2}$ ranging from 0 to $4600 \mathrm{ppm}$ of $\mathrm{CO}_{2}$ preprared out of a reference gas $(2.38 \% \mathrm{CO} 2$ in synthetic air, Westfalen AG, Munster, Germany) to determine the amount of $\mathrm{CO}_{2}$ in our samples. Chromeleon 7.2 Data System Software (Thermo Scientific Waltham, USA) was used to automatize the measurements and process data.

A soil subsample was dried at $40{ }^{\circ} \mathrm{C}$ for 5 days to determine moisture content and phosphorous availability (P-Olsen) using extraction in a $0.5 \mathrm{M} \mathrm{NaHCO}_{3}$ solution and quantification by autoanalyzer (QuAAtro Autoanalyzer, SEAL Analytical Ltd., Southampton, UK). Lastly, we took a fresh soil subsample and measured mineral nitrogen and $\mathrm{pH}$ after $\mathrm{KCl}$ extraction. Briefly, we mixed $10 \mathrm{~g}$ of soil (based on dry weight equivalent) with $50 \mathrm{ml}$ of $1 \mathrm{M} \mathrm{KCl}$ solution in a glass vial and shook it for $2 \mathrm{~h}$. We measured the concentration of mineral nitrogen $\left(\mathrm{NH}_{4}^{+}\right.$and $\left.\mathrm{NO}_{3}^{-}-\mathrm{NO}_{2}{ }^{-}\right)$in the solution using an autoanalyzer (QuAAtro Autoanalyzer, SEAL Analytical Ltd., Southampton, UK). The remaining soil from each pot was kept in a separate bag at $4{ }^{\circ} \mathrm{C}$ to be used for the two feedback phases.

\section{Feedback phase: effects on plant biomass}

The feedback phase to test plant growth responses to the conditioned soils was set up 10 days after the end of the conditioning phase. We divided the soil from each pot in the conditioning phase into two parts to fill two pots of $1.1 \mathrm{~L}$ with the equivalent of $850 \mathrm{~g}$ of dry soil. We covered every pot with aluminium foil to prevent water evaporation and to diminish the number of air-borne propagules that could potentially land in our conditioned soils.

We planted one seedling of each species in "own" and one in "away" soil. After a week, we replanted seedlings in pots where plants had failed to establish. To assess plant growth responses to the different conditioning treatments, we harvested all plants after 6 weeks of plant growth and measured shoot and root biomass after oven drying (70 $\left.{ }^{\circ} \mathrm{C}, 48 \mathrm{~h}\right)$. We chose this relatively short duration of feedback phase to avoid both nutrient limitation and pot size limitation effects on plant growth.

\section{Feedback phase: effects on litter decomposition}

After weighing the dry plant biomass resulting from the conditioning phase, we cut the shoot and root material into small pieces of $1 \times 1 \mathrm{~cm}$ to serve as litter for a decomposition 
experiment. As litter originated from different plant individuals of the same species, we homogenized the resulting litter pieces within each species. Shoot and root litter were kept separately. Subsequently, we sterilized the litter material using gamma irradiation (minimally 25 KGray, Syngenta BV, Ede, the Netherlands). After sterilization, we measured litter $\mathrm{C}$ : $\mathrm{N}$ ratio of shoots and roots of each species as a proxy for litter quality using an elemental analyser (Flash EA 1112, Thermo Fisher Scientific Inc., Waltham, USA).

We used modified $50 \mathrm{~mL}$ centrifuge tubes as microcosms to measure decomposition activity. We added $1.00 \mathrm{~g}$ of dry sterilized litter and inoculated it with conditioned fresh soil ( $0.50 \mathrm{~g}$ of dry weight equivalent). Control microcosms were set up for each of the soils and used to quantify how much $\mathrm{CO}_{2}$ evolved from soil only. Due to the small amount of soil, priming effects were not measured and expected to be negligible. Microcosms were kept at $65 \%$ of the water holding capacity by watering them to pre-determined weight. Water holding capacity was determined for each plant litter type and species separately.

We collected samples for $\mathrm{CO}_{2}$ measurements 11 times during the 48-day incubation (on days $1,2,3,5,7,9,12,17,23,31$ and 47). We tightly closed the tubes and flushed them with $\mathrm{CO}_{2}$-free air for $2 \mathrm{~min}$ at 1 bar. Then, tubes were placed back to the incubator for 4 hours before collecting a $6.2 \mathrm{ml}$ sample of headspace air from each tube using a needle equipped with a pressure lock. We stored headspace air samples in pre-evacuated Exetainer vials (Labco Ltd., Buckinghamshire, UK). Exetainer vials containing $\mathrm{CO}_{2}$ samples were stored at $4^{\circ} \mathrm{C}$ and darkness until their measurement in the gas chromatograph. $\mathrm{CO}_{2}$ concentration in the gas samples were measured using the same setting as for the catabolic response profile. However, for this case we used an additional calibration curve from 0 to $1200 \mathrm{ppm}$ of $\mathrm{CO}_{2}$ in order to increase measurement accuracy of control samples containing only soil with no litter added. At the end of the incubation, we also assessed decomposition by measuring litter mass loss. Dry weights at the start of the incubation experiment were recorded using a 4 decimal scale, as well as on day 48 after freeze-drying the incubation tubes with the remaining materials.

\section{Data analyses}

At the end of the conditioning phase, we analysed the effect of plant identity on soil pH and soil nutrient content within each conditioning type using linear mixed effect models. Plant genera and plant origin were included as fixed factors and block as random effect factor. We then used Tukey post-hoc test to determine significant pairwise differences between plant genera and plant origin. For plant biomass, we tested whether plants grew 
equally well with or without litter using linear mixed models with conditioning type, genera and plant origin as fixed factors and block as a random effect factor. We assessed normality of model residuals using Q-Q plots. We then used pairwise comparisons within each plant species to determine for which plant species, plant conditioning had significant effects on plant biomass. Canoco 5 software was used to conduct multivariate statistics on the catabolic response profiles of the soil communities (Ter Braak, 2012). We calculated the relative mineralization of each substrate and data were log transformed prior to analyses. We used Principal Coordinate Analyses of the dissimilarity matrix based on Bray-Curtis distances to visualize differences between conditioning treatments and plant origin within each plant pair. To test the significance of conditioning and plant origin on the catabolic response profiles, we then performed PERMANOVA analyses (999 permutations) using the 'adonis' function in the 'vegan' package in $R$ ( $R$ Core Team 2017).

After the feedback phase, we analysed the effects of soil conditioning on total plant biomass production within each separate plant genus using linear mixed effect models. Soil conditioning type, plant origin and soil origin (own vs away) were used as fixed effect factors and block was included as a random effect factor. Post-hoc Tukey tests were used to determine significant pairwise interactions. We analysed litter-derived $\mathrm{CO}_{2}$ production from shoot and root litter separately using linear mix model effects. Plant genera, plant origin and soil origin (own vs away) were used as fixed effect factors and block was included as a random effect factor. We also calculated PSF effects as "ln(own/away)" (Brinkman et al. 2010). We calculated PSF effects for both feedback on plant growth and on litter decomposition to assess the relative effects of soils conditioned by the congeneric species compared to soils conditioned by the conspecific in the conditioning phase. Onesample t-test were used to test whether PSF effects significantly differed from 0.

We then determined whether plant biomass responses in the conditioning treatment with plants and litter could be predicted by the average of the responses to conditioning by living plants and plant litter separately. Predicted biomass values were calculated within the five experimental blocks and compared to the observed values. One-sample t-testing was used to test whether differences between observed and predicted biomass significantly differed from 0 . 


\section{Results}

\section{Conditioning phase}

At the end of the conditioning phase, soils that were conditioned by growing plants with or without plant litter had lower availability of all measured nutrients than soils that were conditioned by litter only (Table 4.1). Within each conditioning treatment, plant origin did not have a consistent effect on soil nutrient availability (Table 4.1, Table 4.2).

Table 4.1. Soil $\mathrm{pH}$, soil nitrate and nitrite availability $\left(\mathrm{NO}_{3}+\mathrm{NO}_{2}\right)$, ammonium $\left(\mathrm{NH}_{4}\right)$ and available phosphate $\left(\mathrm{PO}_{4}\right)$ for all plant species and conditioning types at the end of the conditioning phase. RE indicates range-expanding plant species. Values are averages per treatment \pm standard error $(\mathrm{N}=5)$. Letters indicate significant differences between plant genera $(p<0.05)$. Asterisks indicate significant differences between plant origin within plant genera $(p<0.05)$

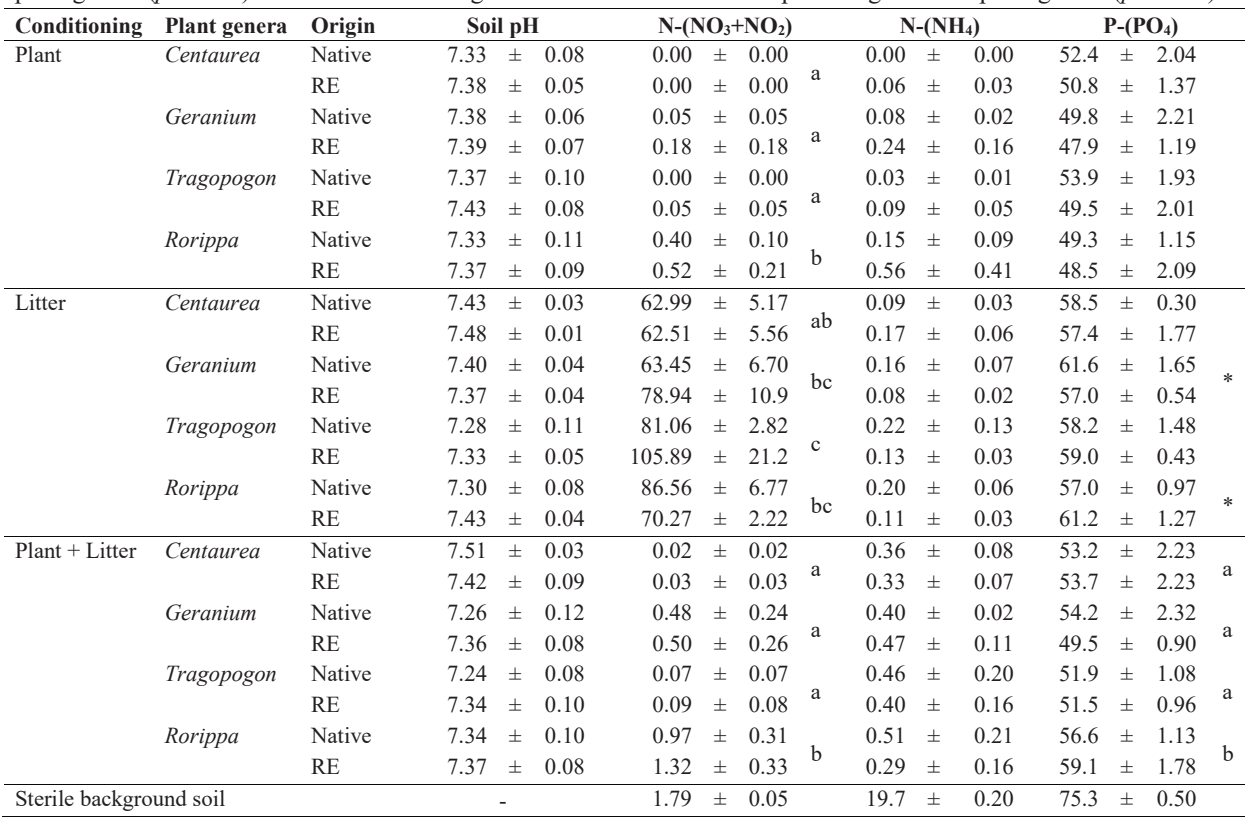


Table 4.2. Linear mixed model analysis of the effects of plant genera and plant origin on soil $\mathrm{pH}$ and nutrient availability after the conditioning phase for the three types of soil conditioning: living plant rhizosphere, decomposing litter, and their combination. Fixed factors: Plant genera $(\mathrm{G})$ and plant origin (O). Response variables: soil $\mathrm{pH}$, soil nitrate and nitrite availability $\left(\mathrm{NO}_{3}+\mathrm{NO}_{2}\right)$, ammonium $\left(\mathrm{NH}_{4}\right)$ and available phosphate $\left(\mathrm{PO}_{4}\right)$.

\begin{tabular}{|c|c|c|c|c|c|c|c|c|c|c|}
\hline \multirow{2}{*}{ Conditioning } & \multirow{2}{*}{ Fixed factor } & \multirow[b]{2}{*}{ num df } & \multicolumn{2}{|c|}{$\mathrm{pH}$} & \multicolumn{2}{|c|}{$\mathrm{N}-\left(\mathrm{NO}_{3}+\mathrm{NO}_{2}\right)$} & \multicolumn{2}{|c|}{$\mathrm{N}-\left(\mathrm{NH}_{4}\right)$} & \multicolumn{2}{|c|}{$\mathrm{P}-\left(\mathrm{PO}_{4}\right)$} \\
\hline & & & F & Signif. & $\mathrm{F}$ & Signif. & $\mathrm{F}$ & Signif. & $\mathrm{F}$ & Signif. \\
\hline \multirow{3}{*}{ Plant } & Genera (G) & 3 & 0.216 & ns & 10.080 & $* * *$ & 1.181 & $\mathrm{~ns}$ & 2.091 & ns \\
\hline & Origin $(\mathrm{O})$ & 1 & 0.632 & ns & 1.104 & ns & 2.518 & ns & 3.715 & . \\
\hline & $\mathrm{G} \times \mathrm{O}$ & 3 & 0.039 & ns & 0.190 & ns & 0.554 & ns & 0.487 & ns \\
\hline \multirow{3}{*}{ Litter } & Genera (G) & 3 & 2.610 & . & 3.829 & $*$ & 0.380 & ns & 0.620 & ns \\
\hline & Origin $(\mathrm{O})$ & 1 & 1.618 & ns & 0.782 & ns & 1.411 & ns & 0.039 & ns \\
\hline & $\mathrm{G} \times \mathrm{O}$ & 3 & 0.740 & ns & 1.849 & ns & 1.144 & ns & 5.917 & $* *$ \\
\hline \multirow{3}{*}{ Plant+Litter } & Genera (G) & 3 & 2.223 & ns & 14.566 & $* * *$ & 0.199 & ns & 7.196 & $* * *$ \\
\hline & Origin $(\mathrm{O})$ & 1 & 0.461 & ns & 0.563 & $\mathrm{~ns}$ & 0.466 & ns & 0.240 & $\mathrm{~ns}$ \\
\hline & $\mathrm{G} \times \mathrm{O}$ & 3 & 0.821 & ns & 0.391 & ns & 0.397 & ns & 1.972 & ns \\
\hline
\end{tabular}

Significance levels “**** $p<0.001 ;$; “**” $p<0.01 ;$;** $p<0.05$; “.” $<<0.1$

Mineral nitrogen: Soil ammonium was generally very low and there were no significant differences between plant genera or plant origin within each conditioning treatment. Soil nitrate availability differed among plant genera within all conditioning treatments (Table 4.1, Table 4.2). Following conditioning with living plants and with living plants in combination with plant litter, soils of Rorippa had higher nitrate availability than the other soils. When conditioned by plant litter, Centaurea soils had the lowest nitrate availability and soil of Tragopogon the highest.

Plant-available phosphorous: conditioning by the combination of living plants and litter resulted in a significant main effect of plant genus (Table 4.2), because soils of Rorippa species had significantly higher phosphorous than soils of the other plant genera (Table 4.1). In the conditioning treatment with litter only, incubation of litter of native Geranium led to higher availability of phosphorous in the soil than litter of range-expanding Geranium, whereas this was opposite for the Rorippa pair (Table 4.1).

Catabolic response profile: at the end of the conditioning phase the catabolic response profile of the soil communities was significantly affected by the type of soil conditioning (Fig. 4.1, Table 4.3). Whether soil was conditioned by plants, litter or a combination, explained 69 $\%$ of the variation in catabolic response profiles (Table 4.3). However, neither plant genus nor plant origin significantly affected the catabolic response profile of the soil community for any of the plant pairs (Table 4.3). 
Plant biomass: For most plant species, plant biomass during the conditioning phase did not differ between treatments with or without litter. However, plant biomass of both Geranium species and the range-expander Centaurea were significantly greatest when plants grew without litter in the soil (Fig. 4.2).

A

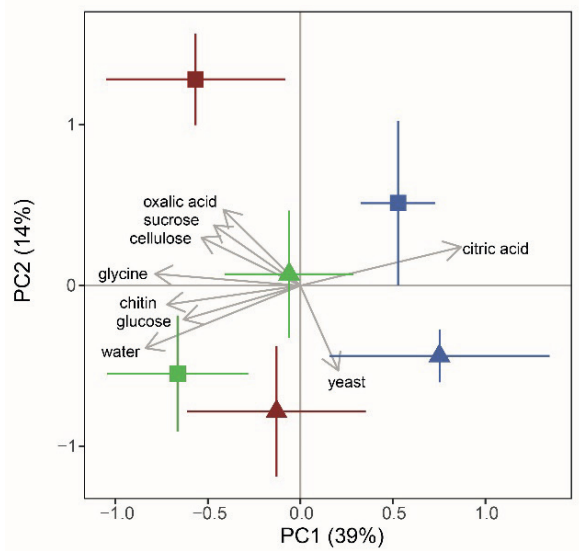

C

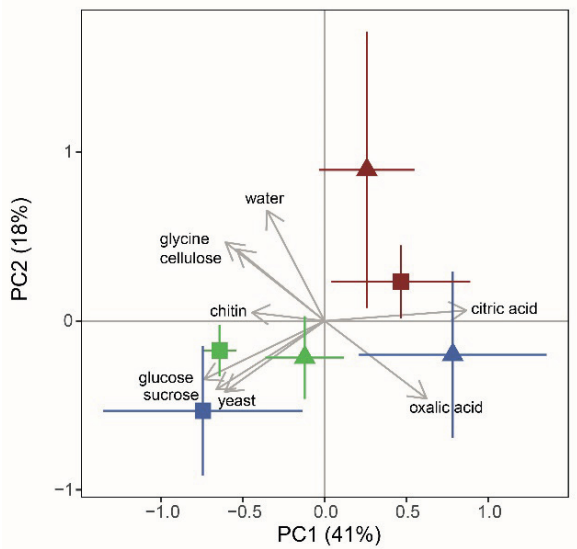

B

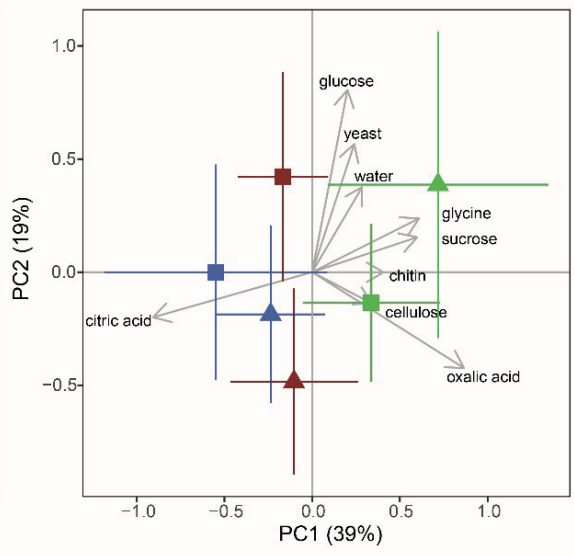

Plant origin

Native

$\Delta \mathrm{RE}$

D

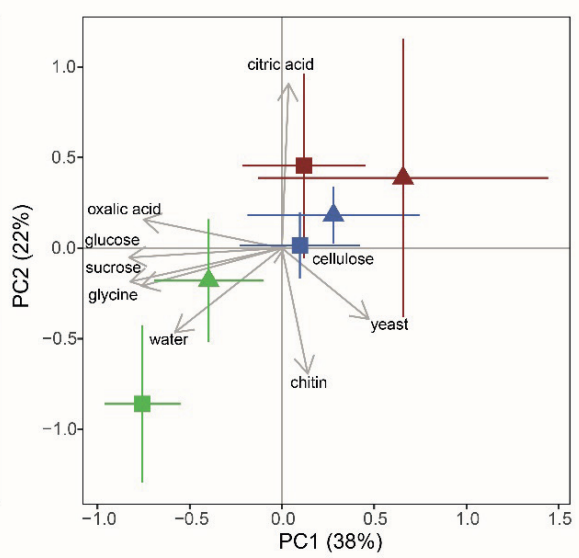

Conditioning

- Plant

- - Litter

- Plant +

Litter

Fig. 4.1. Principal coordinate analyses ordination plots of the catabolic response profiles for each plant pair (A: Centaurea; B: Geranium; C: Tragopogon; D: Rorippa). Circles represent native and triangles represent range-expanding plant species. Different colors indicate the conditioning treatments: plant (green), litter (red), plant + litter (blue). Symbols are centroids and error bars indicate standard errors $(n=5)$. 
Table 4.3. Permanova test (9999 permutations) on Bray-Curtis distance matrix of catabolic response profile data for all plant species and conditioning treatments: living plant rhizosphere, decomposing litter, and their combination. Fixed factors: Plant genera $(\mathrm{G})$, plant origin $(\mathrm{O})$ and soil conditioning type (C).

\begin{tabular}{lrrrrrr}
\hline & Df & SS & MS & F & $\mathrm{R}^{2}$ & p-value \\
\hline Plant genera (G) & 3 & -0.005 & -0.002 & -0.165 & -0.001 & 0.966 \\
Plant origin (O) & 1 & 0.007 & 0.007 & 0.802 & 0.002 & 0.442 \\
Conditioning type (C) & 2 & 2.177 & 1.088 & 119.620 & $\mathbf{0 . 6 9 6}$ & $\mathbf{0 . 0 0 0}$ \\
G x O & 3 & 0.007 & 0.002 & 0.254 & 0.002 & 0.853 \\
G x C & 6 & 0.050 & 0.008 & 0.908 & 0.016 & 0.536 \\
Ox C & 2 & 0.001 & 0.000 & 0.053 & 0.000 & 0.889 \\
G x O x C & 6 & 0.017 & 0.003 & 0.319 & 0.006 & 0.910 \\
Residuals & 96 & 0.874 & 0.009 & & 0.279 & \\
Total & 119 & 3.128 & & & 1.000 & \\
\hline
\end{tabular}

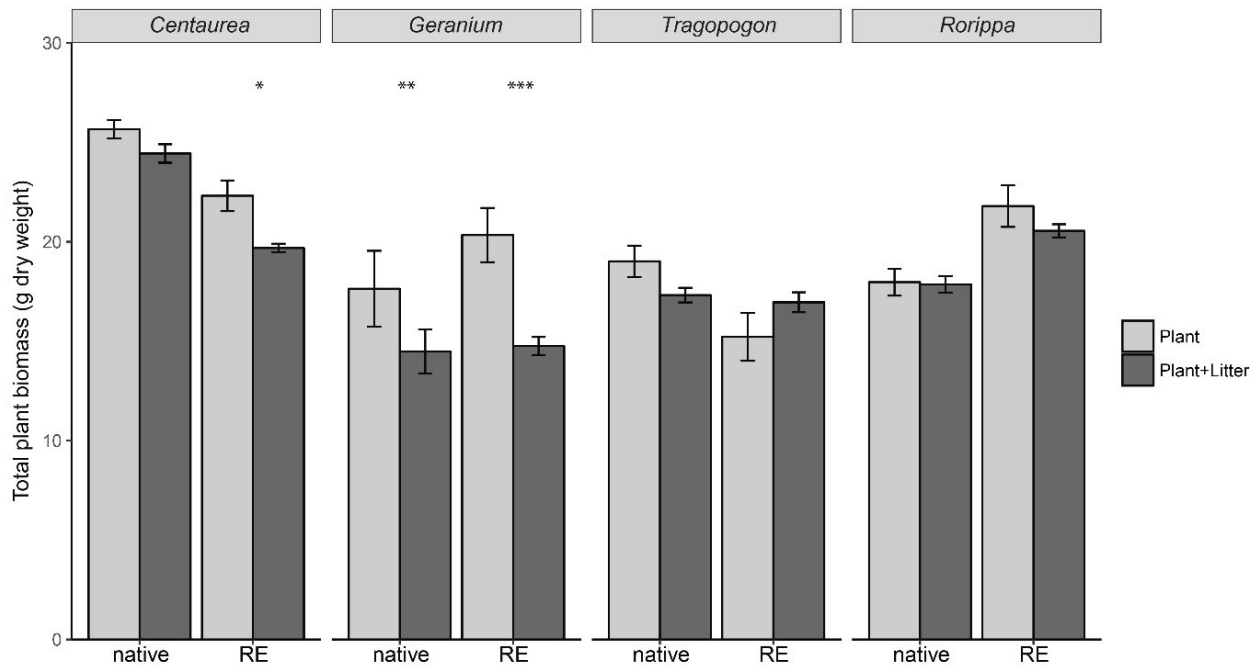

Fig. 4.2. Total plant biomass at the end of the conditioning phase for the soils conditioned by living plants without litter (Plant) and with litter (Plant + Litter). Error bars indicate standard error $(n=5)$. Asterisks indicate significant differences between plant biomass in the conditioning treatment with only plants and plant biomass in the conditioning treatment with both plants and litter within plant species (significance levels: ${ }^{* * *} \mathrm{p}<0.001 ;{ }^{* *} \mathrm{p}<0.01 ;{ }^{*} \mathrm{p}<0.05$ ). 


\section{Feedback effects on plant biomass}

Conditioning by plants, litter, or both: Plants of all plant genera produced more biomass in the soils conditioned by plant litter than conditioned by living plants and by plants plus litter (Fig. 4.3, Table 4.4). Range-expanding Centaurea and Geranium produced significantly more biomass than their congeneric natives in soils that had been conditioned by plant litter (Fig. 4.3A-4.3B, Table 4.4; Conditioning $\times$ Origin interaction). Although both Centaurea species seem to grow best in soil conditioned by plants plus litter of the rangeexpanding Centaurea (Conditioning $\times$ Origin $\times$ Soil interaction (Fig. 4.3A)), this effect was not significant in the pot-hoc test. Tragopogon species produced more biomass in litterconditioned than in plant-conditioned soils, while plant biomass in the combined conditioning treatment was intermediate (Fig. 4.3C). For Rorippa, the main effects of soil conditioning and plant origin on plant biomass were not significant in the post-hoc test (Fig. 4.3D; Table 4.4).

Conditioning by own versus away: There were no significant effects of soil conditioning by "own" versus "away" soils (Table 4.4). Only in the case of Centaurea, there was a significant interaction between the three factors (Conditioning $\times$ Origin $\times$ Soil). This interaction is mainly driven by conditioning by plants plus litter, which led to enhanced biomass production in the soils of range-expanding Centaurea than in the soils of the native Centaurea (Fig. 4.3A). In most cases, PSF effects of total plant biomass did not significantly differ from 0 in any case, indicating neutral PSF effects. There was only one exception of a negative PSF when the range-expanding Geranium grew in soils conditioned by plant plus litter (Fig. S4.1). 

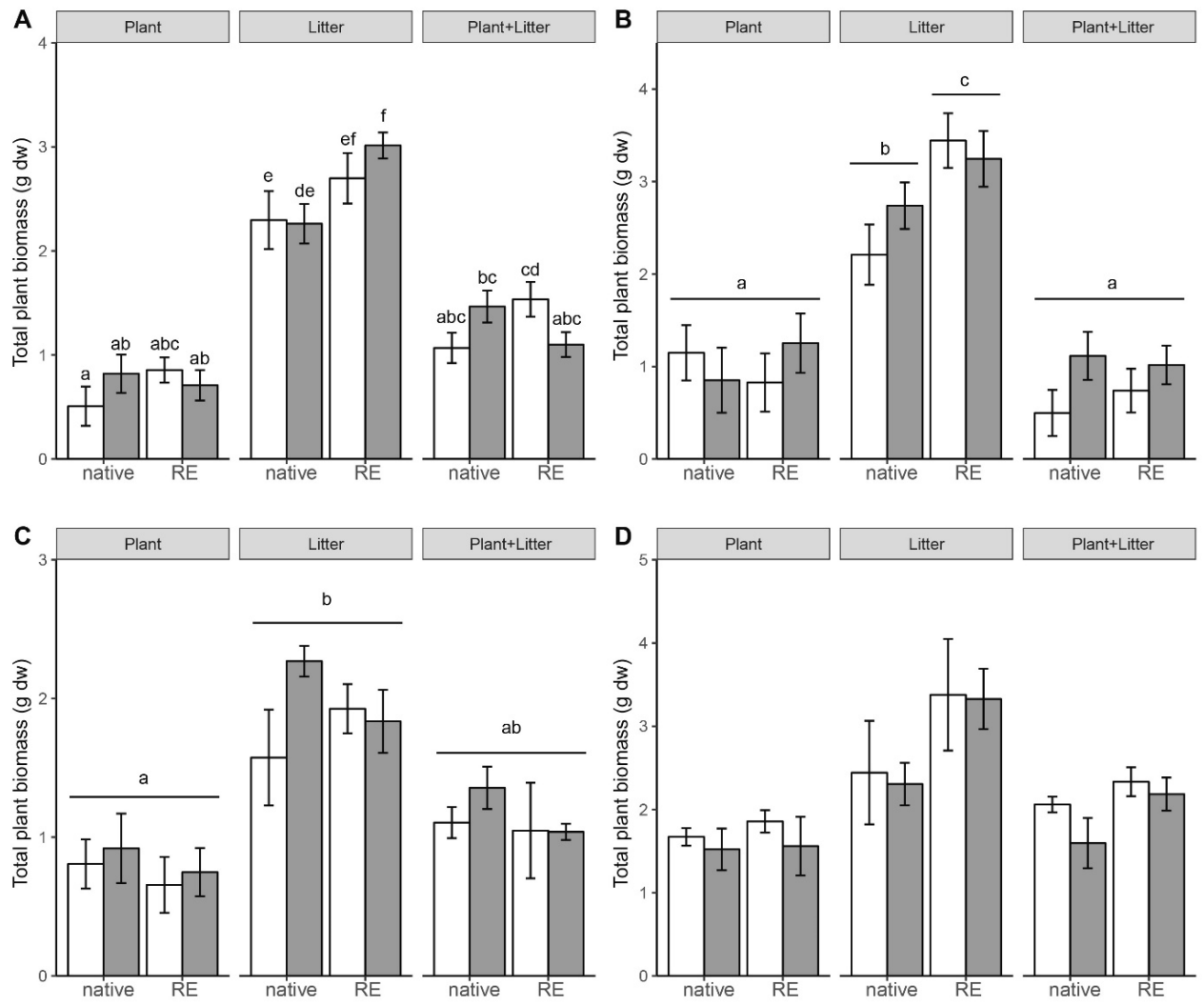

Fig. 4.3. Total plant biomass at the end of the feedback phase for the native and range-expanding (RE) plant species (A: Centaurea; B: Geranium; C: Tragopogon; D: Rorippa) grown in soils conditioned by themselves ("own", white bars) or soils conditioned by the congeneric species ("away", grey bars) and for the three types of soil conditioning (plants, litter, plant + litter). Different letters indicate significant differences $(p<0.05)$ between native and range-expanding plant biomass and between conditioning treatment type (plant, litter, plant + litter). Bars are averages and error bars indicate standard error $(n=5)$. 
Table 4.4. Linear mixed model of the effects of soil conditioning treatment (living plant rhizosphere, decomposing litter, and their combination), plant origin (native and range expander) and soil origin (own and away) on total plant biomass at the end of the feedback phase for the four different plant genera. Fixed factors: Conditioning type (C), plant origin (O) and soil conditioning $(\mathrm{S})$.

\begin{tabular}{|c|c|c|c|c|}
\hline \multirow{2}{*}{ Plant genera } & \multirow{2}{*}{ Fixed factor } & \multicolumn{3}{|c|}{ Total plant biomass } \\
\hline & & $\mathrm{df}$ & $\mathrm{F}$ & Significance \\
\hline \multirow{7}{*}{ Centaurea } & Conditioning $(\mathrm{C})$ & 2 & 163.131 & $* * *$ \\
\hline & Origin $(\mathrm{O})$ & 1 & 8.912 & $* *$ \\
\hline & Soil (S) & 1 & 0.448 & $\mathrm{~ns}$ \\
\hline & $\mathrm{C} \times \mathrm{O}$ & 2 & 4.309 & $*$ \\
\hline & $\mathrm{C} \times \mathrm{S}$ & 2 & 0.239 & ns \\
\hline & $\mathrm{O} \times \mathrm{S}$ & 1 & 3.34 & . \\
\hline & $\mathrm{C} \times \mathrm{O} \times \mathrm{S}$ & 2 & 4.802 & $*$ \\
\hline \multirow{7}{*}{ Geranium } & Conditioning $(\mathrm{C})$ & 2 & 92.819 & $* * *$ \\
\hline & Origin $(\mathrm{O})$ & 1 & 5.629 & $*$ \\
\hline & Soil (S) & 1 & 2.695 & ns \\
\hline & $\mathrm{C} \times \mathrm{O}$ & 2 & 3.973 & $*$ \\
\hline & $\mathrm{C} \times \mathrm{S}$ & 2 & 0.668 & ns \\
\hline & $\mathrm{O} \times \mathrm{S}$ & 1 & 0.202 & ns \\
\hline & $\mathrm{C} \times \mathrm{O} \times \mathrm{S}$ & 2 & 2.582 & . \\
\hline \multirow{7}{*}{ Tragopogon } & Conditioning $(\mathrm{C})$ & 2 & 33.74 & $* * *$ \\
\hline & Origin $(\mathrm{O})$ & 1 & 1.256 & ns \\
\hline & Soil $(\mathrm{S})$ & 1 & 2.255 & ns \\
\hline & $\mathrm{C} \times \mathrm{O}$ & 2 & 0.172 & ns \\
\hline & $\mathrm{C} \times \mathrm{S}$ & 2 & 0.31 & ns \\
\hline & $\mathrm{O} \times \mathrm{S}$ & 1 & 1.901 & ns \\
\hline & $\mathrm{C} \times \mathrm{O} \times \mathrm{S}$ & 2 & 1.093 & ns \\
\hline \multirow{7}{*}{ Rorippa } & Conditioning $(\mathrm{C})$ & 2 & 13.072 & $* * *$ \\
\hline & Origin $(\mathrm{O})$ & 1 & 6.455 & $*$ \\
\hline & Soil (S) & 1 & 1.13 & ns \\
\hline & $\mathrm{CxO}$ & 2 & 1.594 & ns \\
\hline & $\mathrm{C} \times \mathrm{S}$ & 2 & 0.073 & ns \\
\hline & $\mathrm{O} \times \mathrm{S}$ & 1 & 0.038 & ns \\
\hline & $\mathrm{C} \times \mathrm{O} \times \mathrm{S}$ & 2 & 0.107 & ns \\
\hline
\end{tabular}

Significance levels “***” $p<0.001$; “**” $p<0.01$; “*” $p<0.05$; “.” $p<0.1$

\section{Prediction of plant biomass responses}

In most cases, plant biomass in the soils conditioned by plants plus litter was not significantly different from the average of the biomass in soils conditioned by plants and in soils conditioned by litter (Fig. 4.4). Therefore, in most cases observed total plant biomass in soils conditioned by plants plus litter could be predicted by averaging the biomass of the individual plant and litter conditioning treatments. However, the predicted biomass was significantly greater than the measured plant biomass in case of the range 
expander Geranium (in all soils) and both range-expander and native Centaurea (growing in the soil of native Centaurea). Native Geranium follows the same trend as the range expander, however, not significant ( $p 0.055$ and 0.080 for away and own soil, respectively).

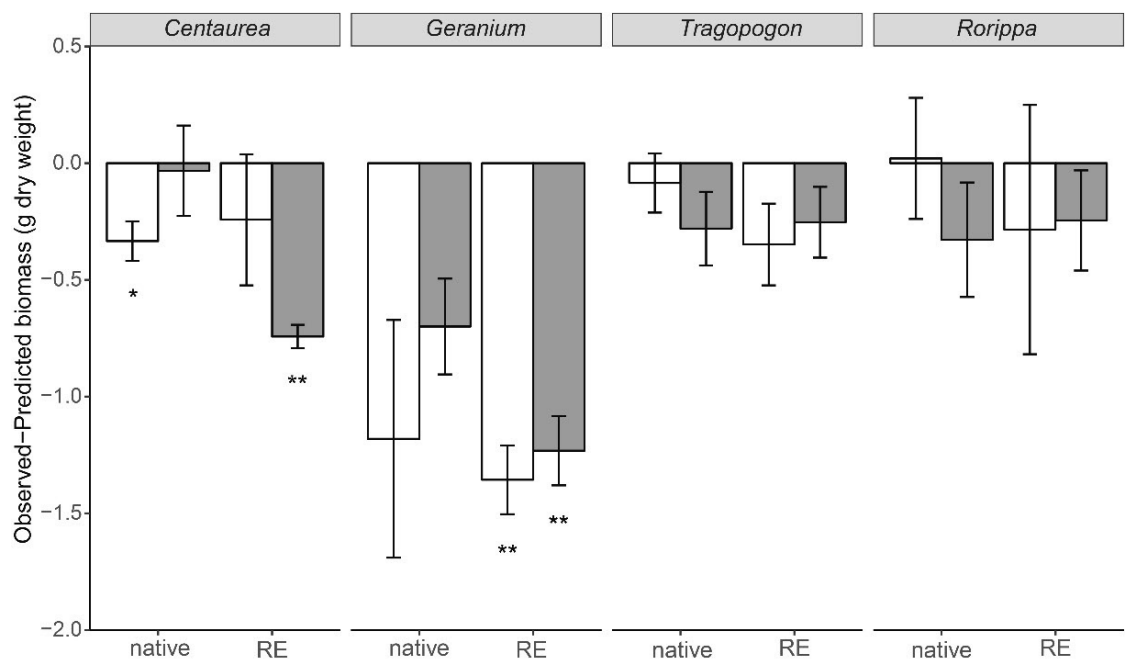

Fig. 4.4. Difference between observed plant biomass in the conditioning treatment with plant and litter and plant biomass predicted from the average of the individual conditioning treatments of plants and litter. Plants were grown in "own" conditioned soils (white bars) and in "away" soils conditioned by the congeneric species (grey bars). Bars are averages $(n=5)$ and error bars indicate standard error. Asterisks indicate significant difference from 0 tested with one-sample t-test. Significance levels: ${ }^{* * *} \mathrm{p}<0.001 ;{ }^{* *} \mathrm{p}<0.01 ;{ }^{*} \mathrm{p}<0.05$.

\section{Feedback effects on litter decomposition}

Litter carbon to nitrogen ratio differed among plant genera (Fig. S4.3). Shoot litter of rangeexpander Geranium and root litter of range-expander Centaurea and Tragopogon had lower carbon to nitrogen ratio than their congeneric natives. This was opposite for root litter of Geranium, while no differences were measured for the Rorippa plant pair (Fig. S4.2). Plant origin had a significant effect on both shoot and root litter decomposition, however the direction of that effect varied among plant genera as indicated by the significant plant genus $\times$ plant origin interaction (Fig. 4.5, Table 4.5). For instance, both shoots and roots of range-expander Centaurea decomposed more than shoots and roots of its congeneric native. This effect was opposite for Tragopogon, where both shoots and roots of the rangeexpander decomposed less than shoots of its congeneric native. In the case of Rorippa species, shoots of the range-expander decomposed more than shoots of the congeneric 
native, however, this was the opposite for root decomposition. Decomposition of plant shoots did not depend on soil conditioning by native or range expander (Fig. 4.5A, Table 4.5). Furthermore, roots of range-expanders decomposed more in soils conditioned by litter of natives than of their own (Table 4.5; Origin $\times$ Soil interaction). Furthermore, we show that litter breakdown measured by litter-derived $\mathrm{CO}_{2}$ and by differences in weight (mass loss \%) yielded comparable insights, as both variables have a strong linear relationship (Fig. S4.3, Fig. S4.4). When we calculated "PSF" for litter decomposition, PSF effects were not significantly different from zero indicating that litter does not decompose differently in soils conditioned by conspecific versus congeneric species (Fig. S4.5).
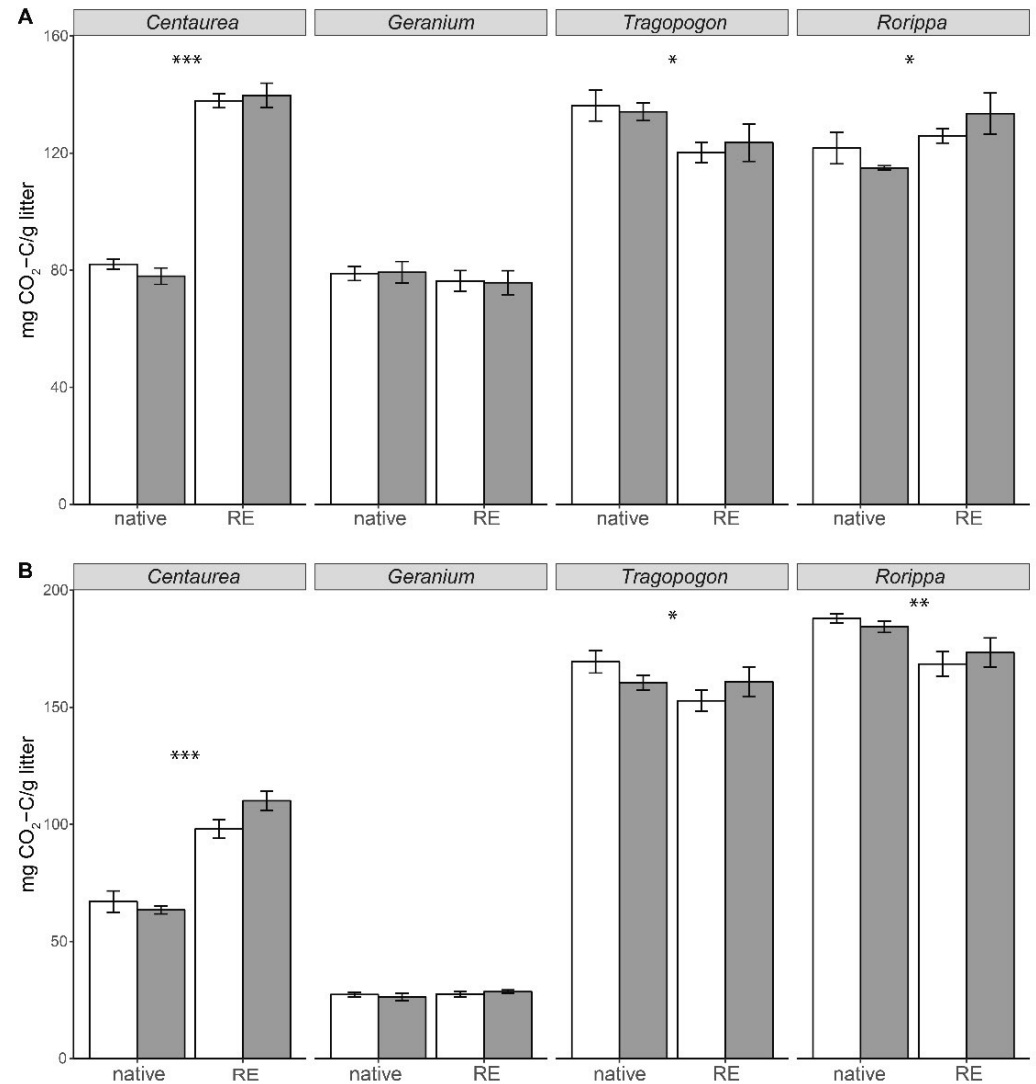

Fig. 4.5. Cumulative $\mathrm{CO}_{2}$ mineralization from the litter incubations of shoots (A) and roots (B) of all the native and range-expanding (RE) plant species with soils conditioned by their own species ("own", white bars) or by the congeneric species ("away", grey bars) and over a period of 48 days. Bars are averages $(n=5)$ and error bars indicate standard error. Asterisks indicate significant differences between native and range-expanding species within plant genera (significance levels: *** $\left.\mathrm{p}<0.001 ;{ }^{* *} \mathrm{p}<0.01 ;{ }^{*} \mathrm{p}<0.05\right)$. 
Table 4.5. Linear mixed model of shoot and root decomposition at the end of the feedback phase on decomposition. Fixed factors: Genera (G), plant origin (O: native versus range expander) and soil conditioning (S: own versus away.

\begin{tabular}{|c|c|c|c|c|c|c|}
\hline \multicolumn{4}{|c|}{ Shoot litter } & \multicolumn{3}{|c|}{ Root litter } \\
\hline Fixed factors & $\mathrm{df}$ & $\mathrm{F}$ & sign. & $\mathrm{df}$ & $\mathrm{F}$ & sign. \\
\hline Genera $(G)$ & 3 & 131.28 & $* * *$ & 3 & 1342.97 & $* * *$ \\
\hline Origin $(\mathrm{O})$ & 1 & 44.81 & $* * *$ & 1 & 4.73 & * \\
\hline Soil (S) & 1 & 0 & ns & 1 & 0.33 & $\mathrm{~ns}$ \\
\hline $\mathrm{G} \times \mathrm{O}$ & 3 & 62.77 & $* * *$ & 3 & 39.61 & $* * *$ \\
\hline $\mathrm{G} \times \mathrm{S}$ & 3 & 0.03 & ns & 3 & 0.32 & ns \\
\hline $\mathrm{O} \times \mathrm{S}$ & 1 & 2.35 & ns & 1 & 8.04 & ** \\
\hline $\mathrm{G} \times \mathrm{O} \times \mathrm{S}$ & 3 & 0.6 & ns & 3 & 0.82 & $\mathrm{~ns}$ \\
\hline
\end{tabular}

\section{Discussion}

We disentangled feedback effects from living plant roots and decomposing litter, while analysing responses of climate warming-induced range-expanding and congeneric native plant species to themselves and each other. Plant-soil feedbacks are important drivers of plant community composition and dynamics (Bever et al. 1997, Kardol et al. 2006) and may be caused by biotic and abiotic changes in the soil induced in the rhizosphere of living plants and by decomposing plant litter. Comparing PSFs of range-expanding and congeneric co-occurring native plant species to themselves and each other will help to understand and predict the establishment success and the ecological consequences of climate-warming induced range shifts, and this knowledge may also help to understand how introduced exotic plant species will influence their new environments (Eppinga et al. 2011, van der Putten et al. 2016).

In support of our first hypothesis, the growth of range-expanders in soils conditioned by living conspecifics was not different from soils conditioned by the living congenerics (Fig. 4.3). This supports the view that, indeed, in the new range range-expanders do not have highly specialized soil biota (Van Grunsven et al. 2010). However, opposite to our first hypothesis, native plant species did not have negative PSF either (Fig. 4.3). These neutral PSF effects of both natives and range expanders appear to contrast with other studies in the new range showing that range-expanders experience less negative feedback than native species (Engelkes et al. 2008). This different result can be explained by the highly conservative "away" treatment that we have used in the current study, as we compared each species in soil conditioned by its own with soil conditioned by a plant species from 
the same genus. In other studies, the "away" treatment usually consists of soil conditioned by plant species from other genera (see e.g. Engelkes et al. (2008)). We have used a socalled congeneric-constrained comparison as suggested by Anacker et al. (2014) and according to our results, PSF through rhizosphere legacies may not help to explain the successful establishment of range-expanding plant species in the new range. Yet, the feedback phase in our experiment was relatively short (van de Voorde et al. 2012). Ultimately, it will be important to test PSF of range-expanding species and the neighboring plant community in the field to be able to predict when and where PSF can play a role in the establishment of range-expanding plant species.

According to our second hypothesis, we expected all plants to perform best in soils conditioned by litter of range-expanders, because litter of exotics in general is associated with higher decomposition rates and nutrient availability (Ehrenfeld et al. 2005, Eppinga et al. 2011). However, our results show that plant biomass of range-expanders and congeneric natives was not significantly different in soils conditioned by litter from either range expanders or natives (Fig. 4.3). As a result, PSF effects from litter of range expanders and congeneric natives were both neutral (Fig. S4.1). The results are in line with the availability of nitrogen in the soil, which was overall high but not significantly different between soils that received range-expander or native litters. In a previous study on plant range shifts it was shown that soils with litter from exotics had enriched available nutrients compared to related natives (Meisner et al. 2012), however, some of those range expanders were from inter-continental origin. The only overlapping plant genus, Rorippa, showed identical results in both studies.

In soils conditioned by plant litter, range-expanders Centaurea and Geranium produced more biomass than their congeneric natives (Fig. 4.3). This indicates that some rangeexpanders, as exotic species, may be more efficient in nutrient utilization than natives (Liu and van Kleunen 2017), which might provide them with a competitive advantage over natives. However, to conclude if that is actually the case both range-expanders and natives need to be grown in competition. It has been shown that introduced exotic species that originate from other continents can benefit from the release of soil-borne enemies in high resource environments, where most plants are known to have predominant negative PSF (Blumenthal et al. 2009). Our findings suggest that this may also be the case for plants that expand their range within continents, and thereby it may be interesting to study whether 
synergistic effects on plant biomass may occur when including effects of both soil biota and nutrient availability.

In support of our third hypothesis, the addition of plant litter during soil conditioning partly alleviated nutrient limitation for subsequent plant growth. Biomass of the Tragopogon and Rorippa plant pairs in the combined conditioning treatment did not differ from the prediction based on individual conditionings (Fig. 4.4). These cases suggest that PSF effects from living plants and litter interact additively, rather than synergistically or antagonistically. However, for the range-expander Geranium and both Centaurea species growing in the soil of the native Centaurea, the measured plant biomass was significantly smaller than the predicted biomass (Fig. 4.4). In those cases, PSF effects from living plants and litter in combination decrease subsequent plant growth disproportionally. Both Geranium species and the native Centaurea may have synergistic negative PSF effects from living plants and litter (Fig. S4.1), as it has been shown previously for other plant species (Zhang et al. 2016b). Alternatively, it may also be that biomass production of these species is disproportionately benefiting from the individual litter and plant conditioning. Interestingly, during the conditioning phase, biomass of Geranium was already smaller when grown in soils that contained own plant litter compared to the control without litter (Fig. 4.2), suggesting that a synergistic negative effect may be a more plausible explanation for the biomass production in the feedback phase.

The small biomass of Geranium species grown in combination with litter in the soil could be due to negative effects of phyllosphere microorganisms (Whitaker et al. 2017), to autotoxicity effects from conspecific litter via self-DNA (Mazzoleni et al. 2015), or from allelopathic compounds released during decomposition (Inderjit et al. 2011). It may also be that the decomposition of Geranium litter caused nutrient limitation to growing plants, because of the immobilization of available nutrients by decomposers (Güsewell and Gessner 2009). Although these effects appeared to be plant species-specific in our experiment, understanding how PSF drives plant community dynamics may require experiments testing the consequences of PSF incorporating both, rhizosphere and litter, as soil conditioning elements.

Opposite to our fourth hypothesis, decomposer communities conditioned by litter of natives did not appear to decompose litter from natives more than from the congeneric range-expanding species (Fig. S4.5). Possibly, the plant species used do not benefit from specialized decomposers. Alternatively, our results do not exclude the possibility that 
during range-expansion plant species remain connected to their specialized decomposers. However, the close relationship between the natives and range expanders does not exclude the possibility that the decomposer communities perceives both litter types equally well (Freschet et al. 2012). Thus, the plant species used in the present study may not experience as much of an advantage from specialized decomposers as plant species that have very contrasting functional traits (Veen et al. 2015a). While it is difficult to determine the underlying mechanisms, our analyses of the catabolic response profiles of the soil communities suggest that soils conditioned with litter of natives and rangeexpanders were functionally equivalent (Fig. 4.1, Table 4.3). Interestingly, we showed more litter breakdown was for shoots and roots of range-expander Centaurea and shoots of range-expander Rorippa than for their respective related natives (Fig. 4.5). These results suggest that, at least for some plant species, range-expanders have the potential to build up a positive litter feedback which could promote their own performance.

\section{Synthesis}

In our experiment, we found that PSF effects both through the rhizosphere of living plants and decomposing litter were neutral for both range-expanders and congeneric related native plant species, which may have been due to our comparison of "own" and "away" conditioned soils within plant genera. Therefore, our results suggest that PSF effects from plant rhizospheres may not play a crucial role in the eventual replacement of native plant species by congeneric range-expanders. Nevertheless, in our study, two out of four rangeexpanding plant species benefitted disproportionally from high nutrient availability in the soil when compared to congeneric natives. Therefore, when litter of range expanders also decomposes at higher rates than natives, as was the case with Centaurea and Rorippa (shoots) in our experiment, range expanders may experience an overall positive feedback from litter decomposition. We also found these results to depend on the plant species examined. Finally, neither range-expanders nor natives appeared to be influenced by specialized decomposer communities. In conclusion, our results emphasize the need to consider legacies from soil conditioning by both plant rhizospheres and decomposing plant litter in PSF experiments in order to finally understand how PSF may contribute to the success, or failure, of inter-continental exotic plant invasions and intra-continental range expansions. 


\section{Acknowledgements}

We thank Rosanne van der Linder for her practical assistance. We thank Ciska Raaijmakers and Hans Zweers for their help with the $\mathrm{CO}_{2}$ measurements, and Iris Chardon for the soil chemical analyses. This study was sponsored by the European Research Council Advanced grant ERC-Adv 260-55290 SPECIALS to Wim H. van der Putten. Ciska Veen was sponsored by NWO-VENI grant (863.14.013). 


\section{Supplementary information}
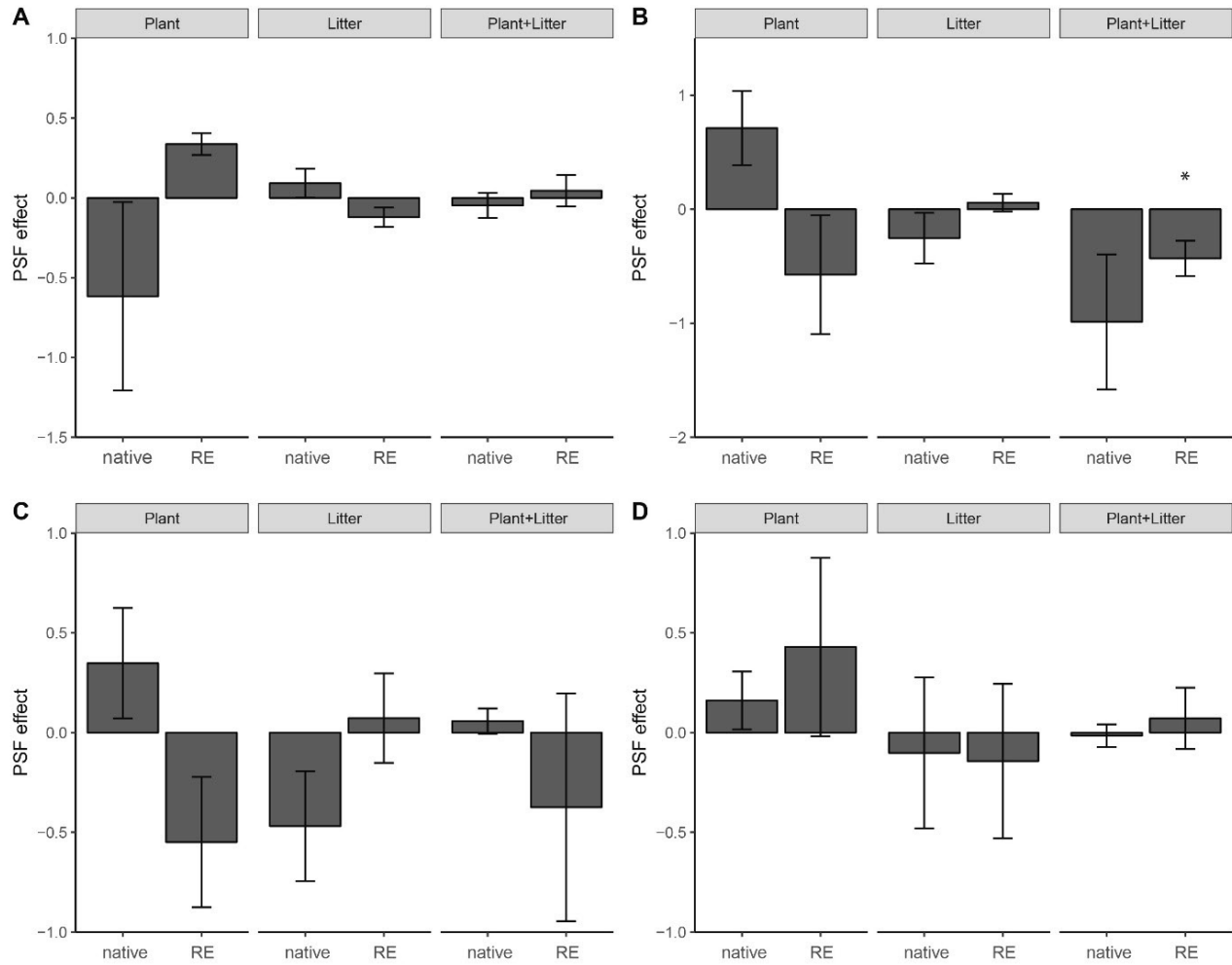

Fig. S4.1. Plant-soil feedback effect $[\ln ($ own/away)] on plant biomass of native and rangeexpanding (RE) plant species of the four genera (A: Centaurea; B: Geranium; C: Tragopogon; D: Rorippa). Bars are averages $(\mathrm{n}=5)$ and error bars indicate standard errors. Asterisks indicate significant difference from 0 tested with one-sample t-test. Significance levels: ${ }^{* *} p<0.001 ;{ }^{* *} p<0.01$; ${ }^{*} \mathrm{p}<0.05$. 

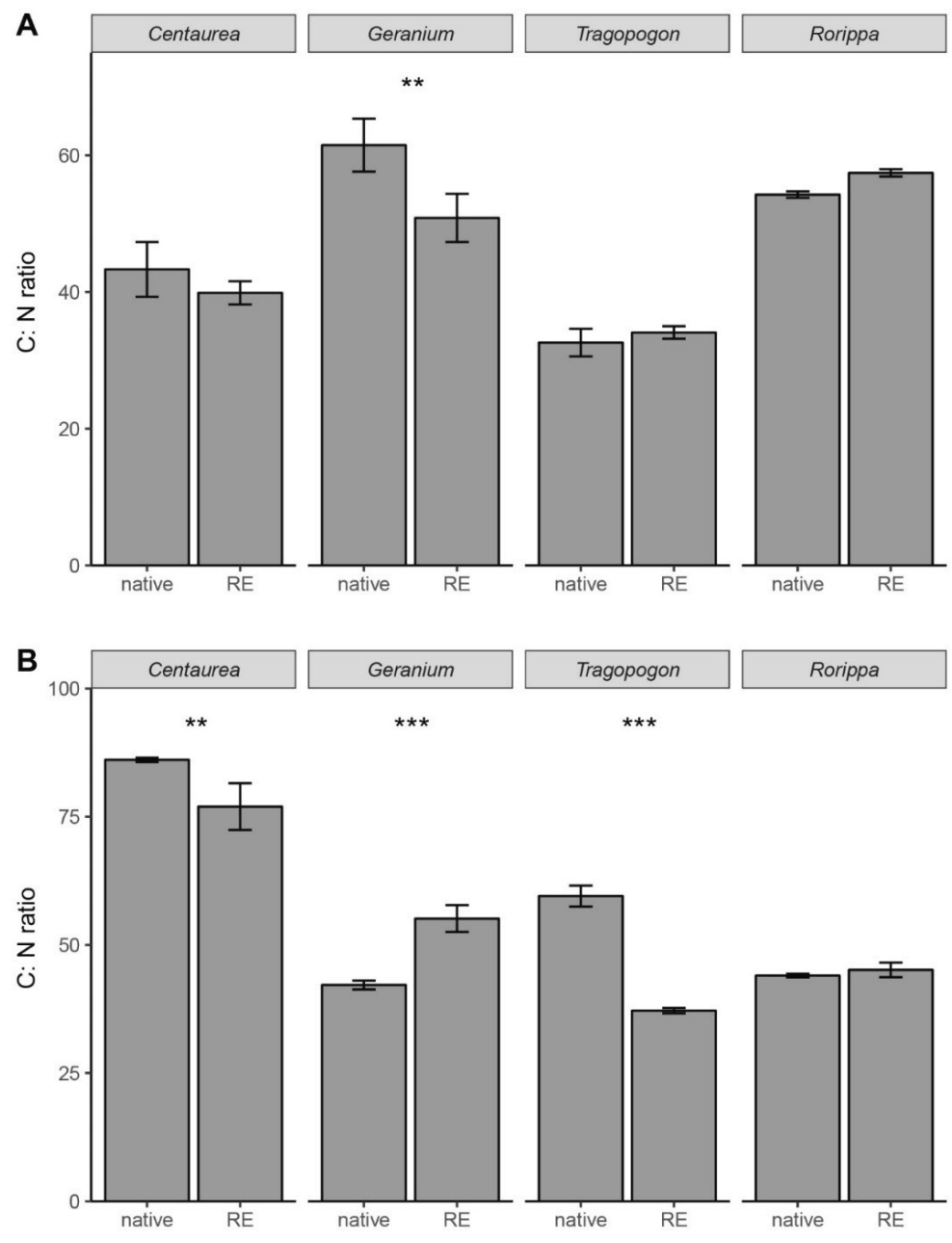

Fig. S4.2. Carbon to nitrogen ratio (C: $\mathrm{N}$ ratio) of shoot (A) and root (B) litter of the native and range-expander (RE) plant species used in the feedback phase to test effects on decomposition. Bars are averages $(n=5)$ and error bars indicate standard error. Asterisks indicate significant differences in litter C: $\mathrm{N}$ ratio between native and range-expander within plant genera. Significance levels: *** $\mathrm{p}<0.001 ;{ }^{* *} \mathrm{p}<0.01 ;{ }^{*} \mathrm{p}<0.05$. 
A

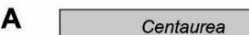

Geranium
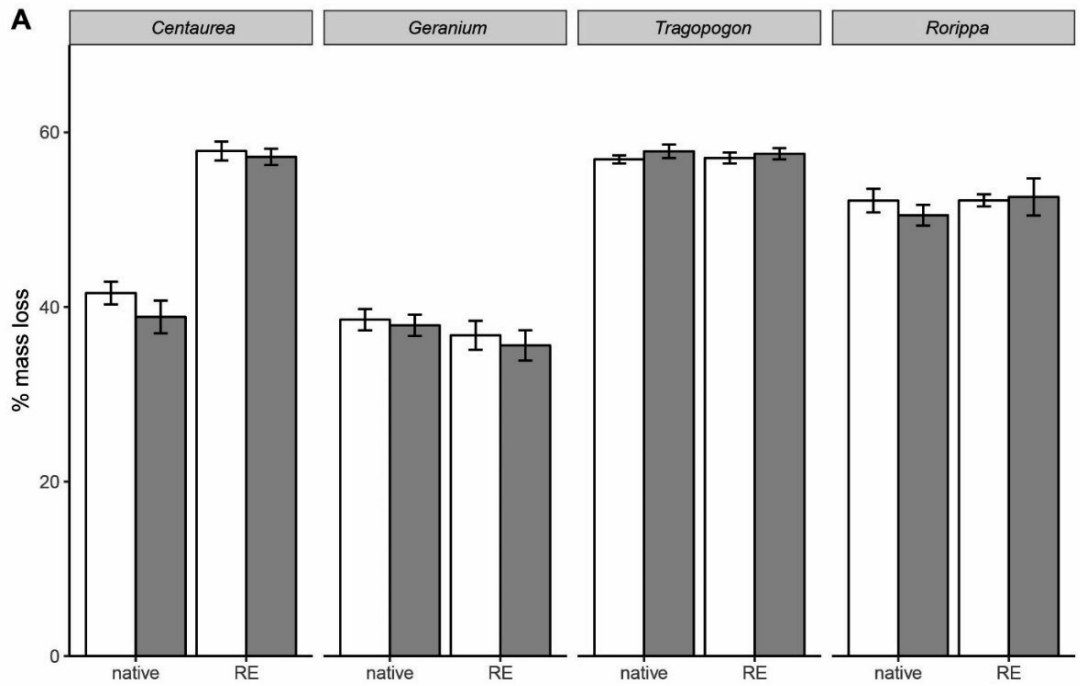

Tragopogon

Rorippa
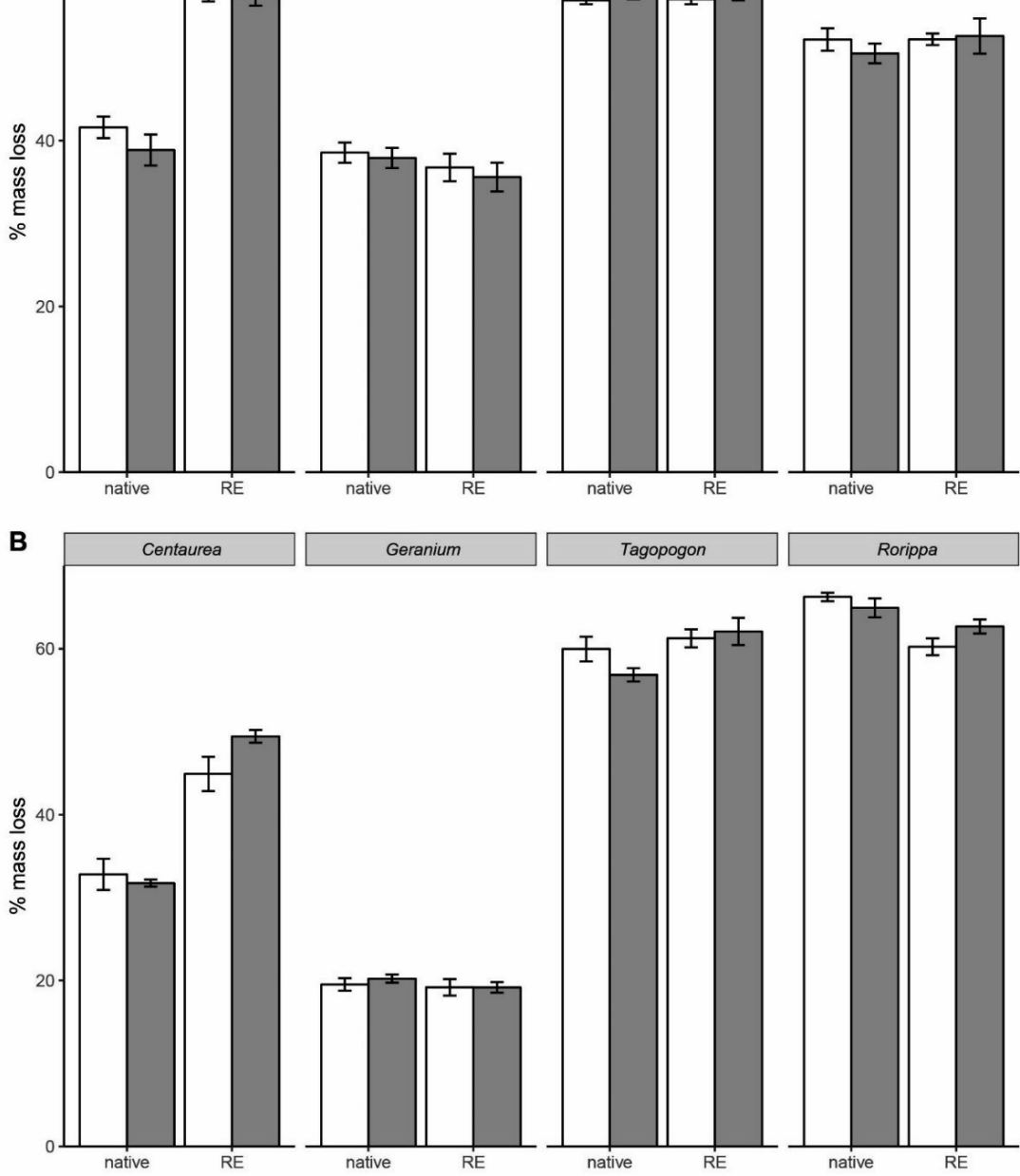

\section{B}

Tagopogon
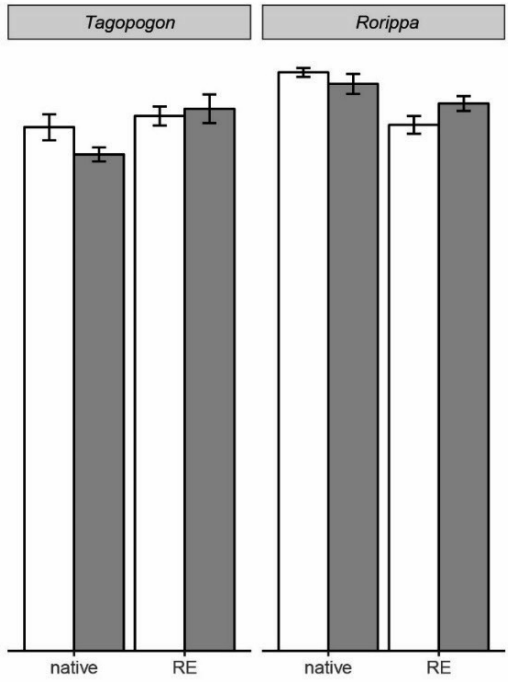

Fig. S4.3. Litter mass loss ( $\%$ of mass loss) at the end of the feedback phase to test effects on decomposition. Each litter was incubated in soils that have been conditioned by the same litter species ("own", white bars) and in soils that have been conditioned by the congeneric plant species ("away", grey bars). Bars are averages $(n=5)$ and error bars indicate standard error. 


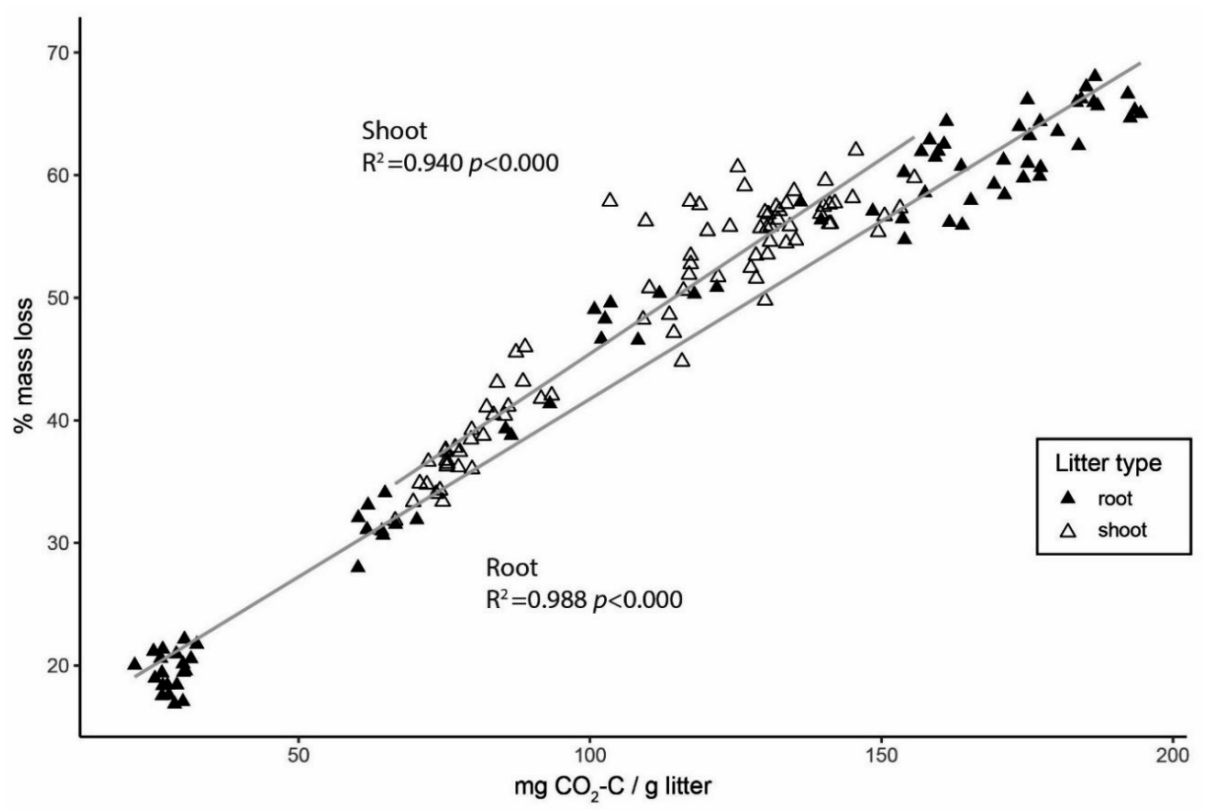

Fig. S4.4. Relationship between cumulative litter-derived $\mathrm{CO}_{2}$ and $\%$ of litter mass loss at the end of the feedback phase on decomposition for shoot (open triangles) and root litter (filled triangles). $\mathrm{R}^{2}$ and p-value of the Pearson correlation test are indicated for shoot and root litter separately. 
A
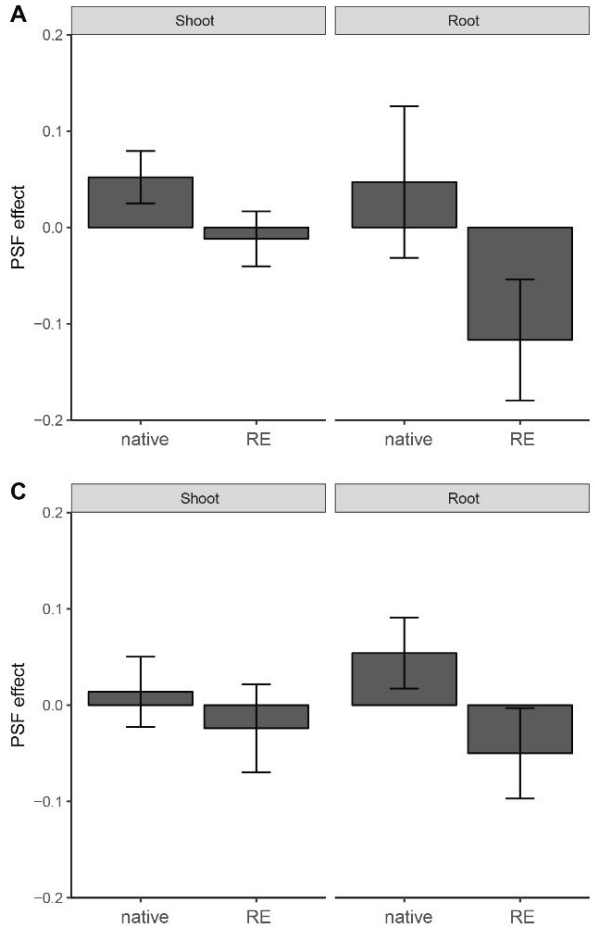

B

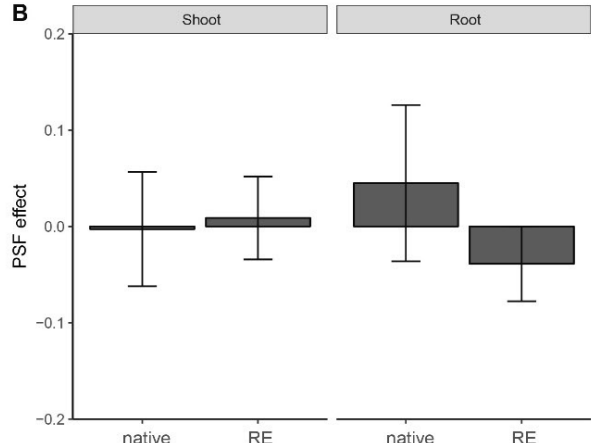

D
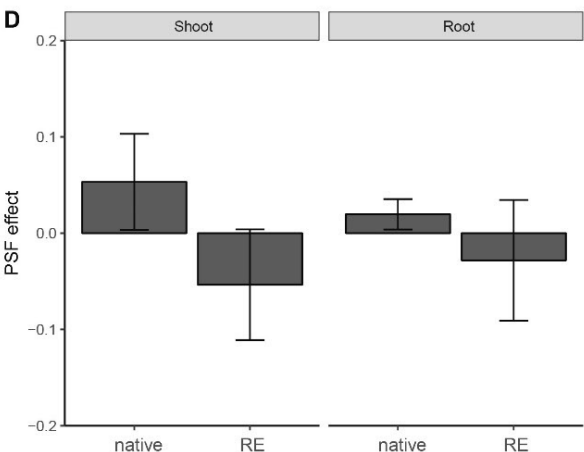

Fig. S4.5. Plant-soil feedback effect [ln(own/away)] on litter decomposition of native and rangeexpanding (RE) plant species of the four genera (A: Centaurea; B: Geranium; C: Tragopogon; D: Rorippa). Bars are averages $(\mathrm{n}=5)$ and error bars indicate standard errors. Asterisks indicate significant difference from 0 tested with one-sample t-test. Significance levels: ${ }^{* *} p<0.001$; $^{* *} p<0.01$; ${ }^{*} \mathrm{p}<0.05$. 


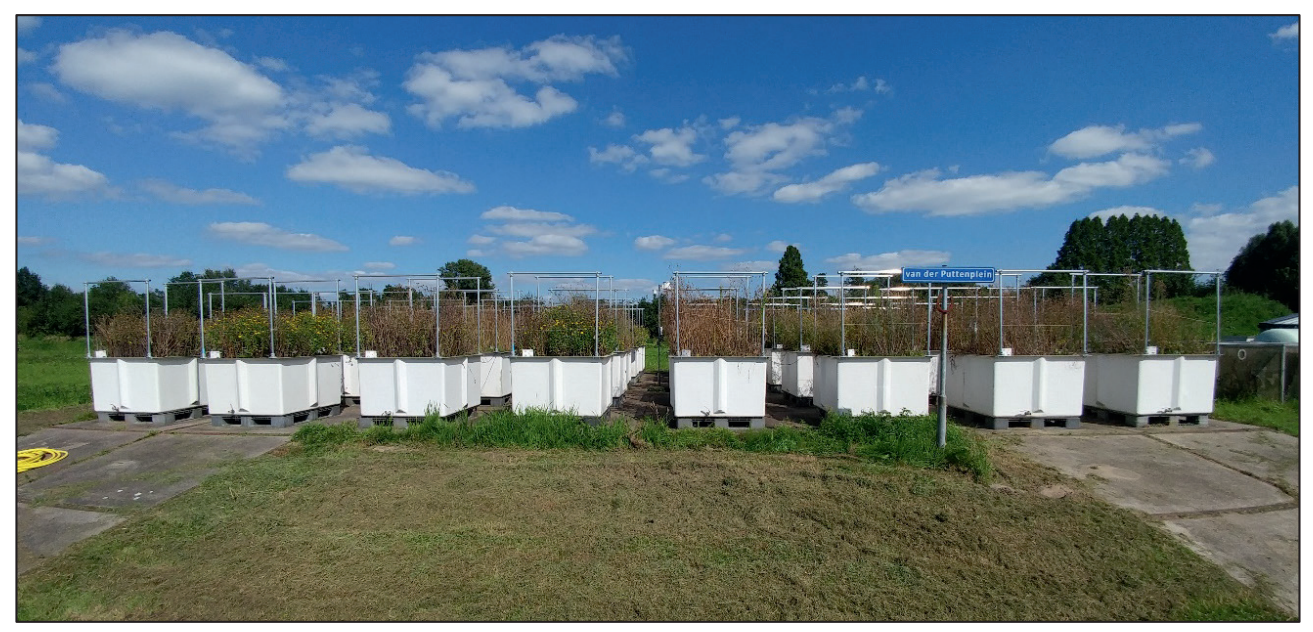




\section{Chapter 5}

\section{Soil functional responses to drought under range-expanding and native plant communities}

Marta Manrubia, Wim H. van der Putten, Carolin Weser, Freddy ten Hooven, Henk Martens, E. Pernilla Brinkman, Stefan Geisen, Kelly S. Ramirez \& Ciska Veen

Manuscript in review 


\section{Abstract}

Current climate warming enables plant species to expand their range to higher latitudes and altitudes. At the same time, climate change increases the incidence of extreme weather events such as drought. While it is expected that plants and soil organisms originating from the south are better able to cope with drought, little is known about the consequences of these range shifts on soil functioning under drought events. Here, we test how rangeexpanding plant species and soil communities drive soil functioning under drought. We performed a full-factorial mesocosm experiment with plant communities of range expanders or related natives, with soil inocula from the novel or the original range, and with and without summer drought. We measured soil functions (litter decomposition, carbon mineralization and enzyme activities), microbial biomass and the relative abundance of soil saprophytic fungi immediately after drought and at 6 and 12 weeks after rewetting.

Drought decreased all soil functions regardless of the plant and soil origin except for soil respiration, which was less hampered in soils of range-expanding plant communities, suggesting a higher resistance to drought. After rewetting, soil functioning responses depended on plant and soil origin. Soils of native plant communities with a history of drought had more litter mass loss and higher relative abundance of saprophytic fungi than soils without drought and soils of range expanders. Soil functions beneath range expanders recovered in a more conservative manner than soils of natives, i.e. without stimulation of litter mass loss above the control rates. At the end of the experiment, most soil functions in mesocosms with drought history did not differ from the control. We conclude that functional consequences of range expansion of plants and soil biota may interact with the effects of drought, and that these effects are most prominent during the first weeks after rewetting of the soil. 


\section{Introduction}

Climate change enables latitudinal and altitudinal range shifts of species from warm to previously colder climate zones (Parmesan and Yohe 2003, Chen et al. 2011, Pauli et al. 2012). This intra-continental movement of species increases the number of introductions of aliens in native ecosystems. For example, in the Netherlands the presence of thermophilic plants typically originating from lower latitudes is increasing in natural ecosystems favoured by mild winter temperatures (Tamis et al. 2005). All plants, including range-expanders, live in association with above and belowground organisms (Wardle et al. 2004, De Deyn and Van der Putten 2005). However, it has been suggested that soil organisms are more limited in their dispersal ability and may not expand their range at the same rate as plants, which may lead to asynchronous introduction of plants and their associated soil organisms in the new range (Berg et al. 2010). Furthermore, it is increasingly acknowledged that although bacteria and fungi are widely present in terrestrial ecosystems, the diversity and composition of soil microbial communities follow global biogeographical patterns (Fierer and Jackson 2006, Tedersoo et al. 2014, DelgadoBaquerizo et al. 2018). As a result plants may encounter a novel soil community in the new range, and together with potential differences in dispersal rates between plants and soil biota, this may lead to temporally disrupted plant-soil interactions (Berg et al. 2010, van der Putten 2012). Previous work has indicated that this may result in the release from plant-specific root pathogens, which can favour the performance of range-expanding plant species in their new habitat (Engelkes et al. 2008, Morriën et al. 2013). At the same time, range-expanding plants may also alter pools and rates of carbon and nutrient cycling via litter effects on soil saprophytic activity (Meisner et al. 2012). However, the functional consequences of plant range expansion and disrupted plant-soil interactions on such ecosystem processes are unknown.

The ecosystems where range-expanding plant species establish are also subjected to other, more direct, effects of climate change such as altered temperature and precipitation regimes. For example, in the Netherlands and other regions of central Europe, the severity and frequency of drought events is projected to increase with current climate change (KNMI 2015, EEA 2016). Drought events can have direct effects on important ecosystem functions and services such as primary productivity, carbon storage and nutrient cycling, both at the scale of ecosystems (de Vries et al. 2012a) and of continents (Ciais et al. 2005). Generally, drought increases the relative abundance of slow-growing taxa in soil 
communities and results in more fungal-dominated food webs (de Vries and Shade 2013, Fuchslueger et al. 2014), because fungal-dominated food webs appear to withstand drought better than bacterial-based food webs (de Vries et al. 2012a, Morriën et al. 2012, Barnard et al. 2013, Worrich et al. 2017). However, severe drought can also alter fungal community composition (Toberman et al. 2008, Meisner et al. 2018), causing loss of decomposition capacity (Setälä and McLean 2004). With ongoing intra-continental species range expansions, direct effects of drought will interact with changes in plant and soil community composition (Bardgett and van der Putten 2014, Classen et al. 2015). Although plant-soil interactions and drought stress are known to affect carbon and nitrogen cycling separately, the consequences of their interactions for soil ecosystem functioning remain poorly understood (Kardol et al. 2010, Sanaullah et al. 2011, Bardgett et al. 2013). Coupling these interactive effects of drought and plant community changes in experiments is crucial for understanding the consequences of plant range shifts (Meisner et al. 2013b) and exotic plant invasions (Caldeira et al. 2015, Alba et al. 2017) on ecosystem functioning.

When in addition to plants, soil organisms expand their range as well, this may modify the impact of range-expanding plant species on ecosystem functioning under drought. This is because the responses of soil microbial processes (e.g. respiration, enzyme activity) to current drought vary in their type and magnitude depending on historical climatic conditions at a regional level (Hawkes and Keitt 2015, Averill et al. 2016, Hawkes et al. 2017). For example, soil communities from Southern Europe that may expand their range have evolved under a typically warm and dry environment, which could affect their response to drought differently from communities that have evolved under cooler and wetter high-latitude conditions. As a result, soil microbial communities originating from dry climate regions are generally more resistant to drought than communities from mesic climate zones (Manzoni et al. 2012). Therefore, the effects of drought on soil functioning could be alleviated by not only the presence of range-expanding plants, but also by the presence of their associated southern soil communities. However, we have poor understanding on ecosystem responses under drought beneath range-expanding plants with or without their own soil community.

The aim of our study was to assess the effects of range-expanding plants and soil biota alone and in combination on soil functional responses to extreme summer drought. We focused on crucial microbial-driven soil functions that support ecosystem productivity and nutrient cycling, such as litter decomposition, soil respiration and extracellular 
enzyme activity, and the abundance of soil saprophytic fungi. We measured these soil functions immediately after drought and upon rewetting to determine the ability of the soil communities to withstand drought (i.e., resistance) and the rate at which communities are able to recover (i.e., resilience), respectively (Pimm 1984, Allison and Martiny 2008, Griffiths and Philippot 2013). We tested the hypotheses that: (1) soils conditioned by range expanding plants are more resistant to drought than soils conditioned by related native plant communities, and (2) soils conditioned by range-expanding plants recover faster after a drought event than soils influenced by related native plant communities. For both hypothesis 1 and 2, we expected that these effects would be stronger when rangeexpanding plants were grown in soils with a southern than with a northern soil community.

To test our hypotheses, we set up a multi-year mesocosm experiment outside with either range-expanding or related native plant communities growing in soils with or without a soil inoculum from the original range of the range-expanding species, i.e., Southern Europe. Two years after the setup of the mesocosms, we simulated a summer drought in half of the mesocosms. In order to assess consequences for ecosystem functioning, we measured litter mass loss, soil respiration, soil enzyme activity, soil microbial biomass and the relative abundance of soil saprophytic fungi at the end of the drought period and at 6 and 12 weeks after rewetting.

\section{Materials and Methods}

\section{$\underline{\text { Mesocosm setup }}$}

In 2013 mesocosms of $1 \mathrm{~m}^{3}$ were set up in the experimental garden of the Netherlands Institute of Ecology (Wageningen, the Netherlands). A total of 40 mesocosms were distributed in 5 rows of 8 mesocosms with a spacing of $0.5 \mathrm{~m}$ between them. Mesocosms were filled with bulk soil collected from a riparian area in Boven-Leeuwen, the Netherlands (51 53' 56.80", 5 33' 45.49").

We inoculated the topsoil $(\sim 20 \mathrm{~cm})$ of the mesocosms with $20 \%$ of field soil inoculum originating from a riparian area in the Netherlands, the expansion range (hereafter, northern soil), or South-East Europe, where the range-expanding plants selected are native (hereafter, southern soil). Northern soil was collected from the Millingerwaard natural area in the Netherlands ( $\left.51^{\circ} 51^{\prime} 56.97^{\prime \prime}, 5^{\circ} 59^{\prime} 33.60^{\prime \prime}\right)$. Southern soil was collected from a floodplain area near Solt in Hungary (46 $47^{\prime} 58.95^{\prime \prime}, 18^{\circ}$ 57' 30.97"). Inoculation soils were 
collected from 5 independent locations in the field (minimum 60 meter apart) and were kept separately to act as experimental replicates in this experiment.

Thereafter, all mesocosms were planted with plant communities of riverine areas formed by range-expanding plant species or related native species. A complete overview of selected range expanders and related native species used can be found in Table S5.1. For the experimental plant communities used in 2015-2016, four of the native plant species were selected for being phylogenetically related to the range-expanding species. The other four species of range expanders did not have a congeneric species in the expansion range, thereby we chose four native plant species with similar traits and life strategies. All seeds were collected from the field in the Netherlands or purchased from an external supplier (Cruydt Hoeck, Nijeberkoop, The Netherlands). In 2015-2016, we planted a total of 64 plant individuals (8 individuals of each plant species) following a regular squared 8x8 grid to ensure balanced plant communities in terms of diversity and evenness of species. Each year at the beginning of the spring, we re-planted annual plants and perennial plants that had died off and removed weedy plant species.

\section{Experimental design}

In summer 2016, we installed rain shelters above all mesocosms. Then, half of the mesocosms were artificially watered two times a week to ensure a rainfall regime representative of the seasonal average precipitation in the area of the last five years (34 litres per week, source: KNMI). The remaining half of the mesocosms received no artificial watering during a period of six weeks in order to mimic an extreme summer drought event (from end of June to $9^{\text {th }}$ August). When the drought phase ended, rain shelters where removed from all mesocosms and we artificially watered the mesocosms when needed to ensure a minimum water input of 34 litres per week for 12 weeks, which is when the last measurements were collected. In total, the experimental period lasted for 18 weeks and was divided in three phases of six weeks each: the experimental drought phase, an early recovery (rewetting phase) and a late recovery phase.

\section{$\underline{\text { Measurements }}$}

\section{Litter decomposition}

Litter mass loss was measured at the end of the three different phases of the experiment using a modified version of the Tea Bag Index method, a standardized protocol to assess decomposition of substrates of contrasting chemical complexity. Instead of a 3-month 
incubation proposed by Keuskamp et al. (2013), we buried tea bags in the soil for a total of 6 weeks corresponding to our experimental phases. At the start of each period of six weeks, we buried pre-weighed green and rooibos tea bags at $8 \mathrm{~cm}$ below the soil surface in each mesocosm. In this way, the chemical composition of the substrates was standardized at the beginning of each phase. The three sets of tea bags were buried in three locations in the centre of the mesocosms avoiding potential edge effects. In each of the mesocosms plants were planted following a regular grid and tea bags were consistently buried beneath the same plant species in either native or range-expanding plant communities. At the end of each six-week phase, we retrieved the teabags from the soil, removed big fragments of roots, oven-dried the remaining tea material ( $70{ }^{\circ} \mathrm{C}$ for $48 \mathrm{~h}$ ), dry sieved it to $0.4 \mathrm{~mm}$ to remove soil particles and weighed it to determine mass loss.

\section{Soil sampling}

At the end of each of the six-week experimental phases, i.e., right after drought, six and twelve weeks after the end of the drought, we collected soil samples. We took a composite soil sample of four different soil cores (2.5 cm diam.; 0-10 cm depth) to obtain a representative soil sample from each mesocosm. Soil samples were immediately sieved through a $4 \mathrm{~mm}$ sieve. Then, a subsample was collected in an Eppendorf tube, immediately frozen in liquid nitrogen and kept at $-80^{\circ} \mathrm{C}$ for molecular analyses. The rest of the soil was kept at $4{ }^{\circ} \mathrm{C}$ and darkness until further analyses, which were performed within a week after soil collection.

\section{Background environmental parameters}

To determine how drought affected soil nutrient availability in the mesocosms, we quantified mineral nitrogen in the soil before the drought event started and at the end of the drought. We extracted soil available nitrogen ( $\mathrm{NO}_{3}^{-}, \mathrm{NO}_{2}^{-}$and $\mathrm{NH}^{+} 4$ ) from all mesocosm by shaking a $10 \mathrm{~g}$ dry weight equivalent in a $50 \mathrm{ml}$ of $1 \mathrm{M} \mathrm{KCl}$ solution for 2 hours. We determined the concentration of mineral nitrogen using an autoanalyzer (QuAAtro Autoanalyzer, SEAL Analytical Ltd., Southampton, UK).

We measured plant biomass in November 2016, corresponding to the end of the growing season, to assess plant productivity under the different experimental treatments (Fig. S5.1). For aboveground biomass, we subsampled a fixed row of eight individual plants per mesocosm containing one individual of each species. For root biomass, we collected three soil cores of $73.6 \mathrm{~cm}^{3}$ each $(15 \mathrm{~cm}$ depth $* 2.5 \mathrm{~cm}$ diameter) and subsequently washed the 
root material contained in each core to have an estimate of standing root biomass in the topsoil.

\section{Soil carbon mineralization and soil microbial biomass}

We measured soil carbon mineralization (basal soil respiration) in all soil samples. We weighed the equivalent of $4 \mathrm{~g}$ of dry soil in $50 \mathrm{~mL}$ centrifuge tubes with a modified lid equipped with a rubber septum and a rubber o-ring in order to ensure air tightness. Tubes were capped tightly and we then flushed the headspace air in the tubes with $\mathrm{CO}_{2}$-free air for 2 min at 1 bar (Westfalen Gassen Nederland BV, Deventer, Netherlands). We incubated all tubes at $20^{\circ} \mathrm{C}$ for $24 \mathrm{~h}$ in a climate-controlled chamber (Economic Lux chamber, Snijders Labs, Tilburg, Netherlands). Subsequently, we took a $6.2 \mathrm{~mL}$ sample of headspace air from each tube using a needle and stored it in pre-evacuated air-tight vial (Labco Exetainer). We determined the concentrations of $\mathrm{CO}_{2}$ in the gas vials (over pressure of 1 bar) by injecting $250 \mu \mathrm{l}$ of each sample in a Trace Ultra GC gas chromatograph equipped with a flame ionization detector with methanizer (mFID) (Interscience BV, Breda, the Netherlands) and a TriplusRSH auto-sampler (Interscience BV, Breda, the Netherlands), and a Rt-QBOND (30 m, 0.32 mm ID) capillary column (Restek, Bellefonte USA). We used helium 5.0 as a carrier gas, a sample split ratio of $1: 20$ and set oven temperature at $50{ }^{\circ} \mathrm{C}$ with a flow of $5 \mathrm{ml}$. We used a calibration curve of known concentrations of $\mathrm{CO}_{2}$ ranging from 0 to $4600 \mathrm{ppm}$ of $\mathrm{CO}_{2}$ prepared out of a reference gas $\left(2.38 \% \mathrm{CO}_{2}\right.$ in synthetic air, Westfalen AG, Münster, Germany) to determine the amount of $\mathrm{CO}_{2}$ in our samples. Chromeleon 7.2 Data System Software (Thermo Scientific Waltham, USA) was used to automatize the measurements and process data.

Subsequently, we also measured substrate-induced respiration, a proxy for soil microbial biomass (Anderson and Domsch 1978; Fierer 2003). After the sampling of headspace gas for soil carbon mineralization, we added $4 \mathrm{~mL}$ of yeast extract solution (12 g yeast / litre) to each tube and incubated them at $20{ }^{\circ} \mathrm{C}$ for $4 \mathrm{~h}$. We then proceeded to sample headspace air and measure its $\mathrm{CO}_{2}$ concentration following the same protocol as for the soil carbon mineralization.

\section{Hydrolytic enzyme activity in the soil}

At the end of each six-week phase, we measured potential activity of $\beta$-glucosidase, acid phosphatase and alanine-aminopeptidase enzymes using fluorometric assays (Baldrian 2009). We obtained soil homogenates by shaking (10 min, $330 \mathrm{rpm}) 1 \mathrm{~g}$ of fresh soil 
suspended in $50 \mathrm{~mL}$ of sodium acetate buffer $(2.5 \mathrm{mM}, \mathrm{pH}=5.5)$. Fluorogenic substrates 4-methylumbellyferyl- $\beta$-D-glucopyranoside (MUFG), 4-methylumbellyferyl-phosphate (MUFP) and L-alanine-7-amido-4-methylcoumarin (AMCA) were commercially obtained (Sigma-Aldrich Chemie N.V. Zwijndrecht, Netherlands). We dissolved all substrates in dimethyl sulfoxide at concentrations of $2.5 \mathrm{mM}$ for AMCA and $2.75 \mathrm{mM}$ for MUFG and MUFP. A $40 \mu \mathrm{l}$ of substrate solution was mixed with $250 \mu \mathrm{l}$ of soil homogenate in each well of a black 96-well plate. Three technical replicates were included per soil sample and enzyme activity. We calibrated concentrations of enzyme product with a dilution curve made from a stable form of the two fluorogenic compounds (1.0 $\mathrm{mM}$ methylumbellyferol and 1.0 mM 7-aminomethyl-4-coumarin). Fluorescence was measured using a plate reader at the start of the incubation and after $2 \mathrm{~h}$ of incubation at $40{ }^{\circ} \mathrm{C}$ with an excitation and emission wavelengths of $360 \mathrm{~nm}$ and $460 \mathrm{~nm}$, respectively (Synergy HT, BioTek Instruments, Winooski, Vermont, USA). We compared the measured fluorescence in our samples, after subtraction of the blank, with the standard dilution curves to calculate the amount of enzymatic product formed over the incubation time. In our study, a unit of enzyme activity is defined as the amount of enzyme reaction product ( $\mu \mathrm{mol})$ per gram of dry soil and hour.

\section{Fungal community and relative abundance of saprophytic fungi}

We extracted DNA from soils samples stored at $-80^{\circ} \mathrm{C}$. Briefly, DNA was extracted from $0.25 \mathrm{~g}$ of soil using the PowerSoil DNA Isolation Kit (Mo Bio Laboratories, Carlsbad, California, USA) following the manufacturer's instructions. We then amplified DNA using duplicate PCR reactions with bar-coded primers for multiplexing and re-identifying individual samples following sequencing. Fungal community composition was determined by targeting the ITS2 region using ITS4 and ITS9 primers (Ihrmark et al. 2012). PCR products were purified using the Agencourt AMPure XP magnetic bead system (Beckman Coulter Life Sciences, Indianapolis, Indiana, USA) with a volume ratio of PCR product to beads of 1: 0.7. Purified PCR products were analysed in a Fragment Analyzer using a Standard Sensitivity NGS Fragment Analysis kit (1bp-6000bp) and following manufacturer's instructions (Advanced Analytical Technologies GmbH, Heidelberg, Germany). Finally, fungal ITS amplicons were sequenced using the Illumina MiSeq platform.

The ITS amplicon reads were analysed using the Hydra pipeline version 1.3.2 (de Hollander 2017) implemented in Snakemake (Köster and Rahmann 2012). Adapter 
sequences and PhiX contaminants were removed using BBDuk2 from the BBMap tool suite (Bushnell 2015). Paired-end reads were merged using the option fastq-mergepairs of vsearch (Rognes et al. 2016). Sequences were converted to FASTA format and concatenated into a single file. All reads were clustered into OTUs using the UCLUST smallmem algorithm (Edgar 2010). Chimeric sequences were detected using the UCHIME algorithm (Edgar 2011). All reads were mapped to OTUs using the usearch-global method implemented in VSEARCH and a OTU Table was created and converted to BIOM-Format (McDonald et al. 2012). Taxonomic information for each OTU was obtained using the RDP Classifier re-trained on the UNITE database 7.2 (Kõljalg et al. 2013). Soil samples with a read number lower than 1000 reads and singletons were not included in further analyses.

Although we collected fungal community composition at OTU level and at different times during the experiment, we have only focused on data at the functional level obtained using the Funguild database (Nguyen et al. 2016). These samples were collected at the same times as the different soil functions were measured in our experiment. In our study we focused on the relative abundance of soil saprophytic fungi with respect to the total fungal community in order to link shifts in functional composition to differences in saprophytic activities measured. Thereby, we calculated the relative abundance of saprophytic fungi by accounting the total number of reads that were assigned to the saprophytic trophic mode (as a single trophic mode or in combination) within the total number of OTU reads in that sample (OTUs with other trophic modes and OTUs with no assigned guild).

\section{Data analyses}

We used general linear mixed effect models to test the effect of the experimental treatments on soil functions (litter mass loss, soil respiration, substrate-induced respiration and enzyme activity), the relative abundance of soil fungi and plant shoot and root biomass. We included plant community origin, soil inoculum and drought as fixed factors and block as random factor. Satterthwaite approximation of denominator degrees of freedom was used with "lmerTest" package in R (R Core Team 2017). Enzyme activity rates, root biomass and relative abundance of saprophytic fungi data were log transformed when needed prior analyses to meet normality assumption in ANOVA as checked with Shapiro-Wilks test. Two extreme outlier data points of phosphatase and aminopeptidase activity were detected using the "outlier.test" function in the "car" package in $\mathrm{R}$ and removed from the analyses ( $R$ Core Team 2017). 
Subsequently, for each variable we calculated the proportional change from the control as a measure of stability of the soil communities during drought (i.e., resistance) and at different stages after drought (i.e., resilience) (Griffiths et al. 2000). Negative values of this proportional change from the control indicate that drought negatively influenced that parameter with respect to the control. Positive values indicate that drought stimulated that parameter over the control levels. This relative difference to the control was always calculated within each of the five independent blocks. We then tested stability differences between plant communities of different origin and soil inoculum using general linear mixed effect models, which did not include the fixed factor "drought". All statistical tests were performed using R (R Core Team 2017).

\section{Results}

\section{Background environmental parameters}

The soil moisture content at the start of the experiment was $20.8 \pm 1.1 \%$. At the end of the six weeks of drought, soil moisture in the drought treatment was reduced to $4.9 \pm 0.6 \%$ while it was $11.9 \pm 1.6 \%$ in the control treatment. After the six weeks period of the early stage recovery, the soil moisture of the drought treatment levelled with that of the control $(13.3 \pm 1.2$ and $13.6 \pm 1.9 \%$, respectively). At the end of the following six weeks period, late stage recovery, soil moisture was $16.1 \pm 1.3 \%$ for the drought mesocosms and $16.4 \%$ for the control.

Soil nitrate availability was affected by plant community origin and soil inoculum before the drought started (Plant: $F_{1,32}=10.7, P=0.002$; Soil: $F_{1,32}=12.4, P=0.001$ ), and by soil inoculum only in the case of ammonium (Soil: $\mathrm{F}_{1,32}=4.3, P=0.044$ ). Before the drought, soils of range-expanding plant communities had more soil nitrate than soils of native communities, and northern soils had more soil nitrate and ammonium than southern soils.

At the end of the drought, soil nitrate availability was negatively affected by drought in soils of native plant communities with respect to the control, but not in soils of range expanders (Soil x Plant: $\mathrm{F}_{1,32}=17.1, P<0.001$ ), and in northern soils with respect to southern soils (Drought $x$ Soil: $\mathrm{F}_{1,32}=5.03, P=0.031$ ). The main effect of soil inoculum remained for ammonium, where availability was higher for northern soils compared to southern soils (Soil: $\mathrm{F}_{1,32}=4.8, P=0.034$ ). Mesocosms subjected to drought had significantly higher availability of ammonium than control mesocosms (Drought: $F_{1,32}=17.9, P<0.001$ ). 
Neither above nor belowground plant biomass were affected by drought, plant community origin or soil inoculum (Fig. S5.1).

\section{Decomposition of high-quality substrate}

Mass loss of high-quality litter, i.e. green tea, was decreased by drought during the dry period (Table 5.1, Table 5.2). After rewetting of the soil (i.e. early-stage recovery), mass loss of high-quality litter tended to be stimulated in soils with a history of drought (Table 5.1, Table 5.2). During late recovery, litter mass loss was significantly higher in soils of native plant communities than in soils of range expanders (Table 5.1, Table 5.2).

The proportional change in litter mass loss in the dry treatment relative to the control in each treatment (i.e., same plant origin and soil inoculum) was negative during drought and decomposition was decreased by $12.9 \%$ on average (Fig. 5.1A). Despite the significant plant and soil interaction (Table 5.3), post-hoc pairwise comparisons of least square means revealed no significant differences between any specific treatments. During early-stage recovery, decomposition in the drought treatment recovered to control values (Fig. 5.1B). Both during drought and early recovery, the proportional change from the control was not affected by plant community origin and soil inoculum (Table 5.3). During the late recovery, there was a significant interaction between plant community origin and soil inoculum, where "home" combinations (i.e., northern soils with native communities or southern soils with range-expanding communities) had a positive proportional change, while this did not occur for "away" combinations (i.e., northern soils with rangeexpanding communities or southern soils with native communities combinations) (Fig. 5.1C, Table 5.3).

\section{Decomposition of low-quality substrate}

Mass loss of low-quality litter, i.e. rooibos tea, was significantly decreased by drought during the dry period (Table 5.1, Table 5.2). During both recovery phases, litter mass loss was stimulated by drought history beneath native plant communities, while it remained negatively affected beneath range-expanding plant communities (Table 5.1, Table 5.2). Furthermore, mass loss was higher in southern soils than northern soils (Table 5.1, Table $5.2)$. 


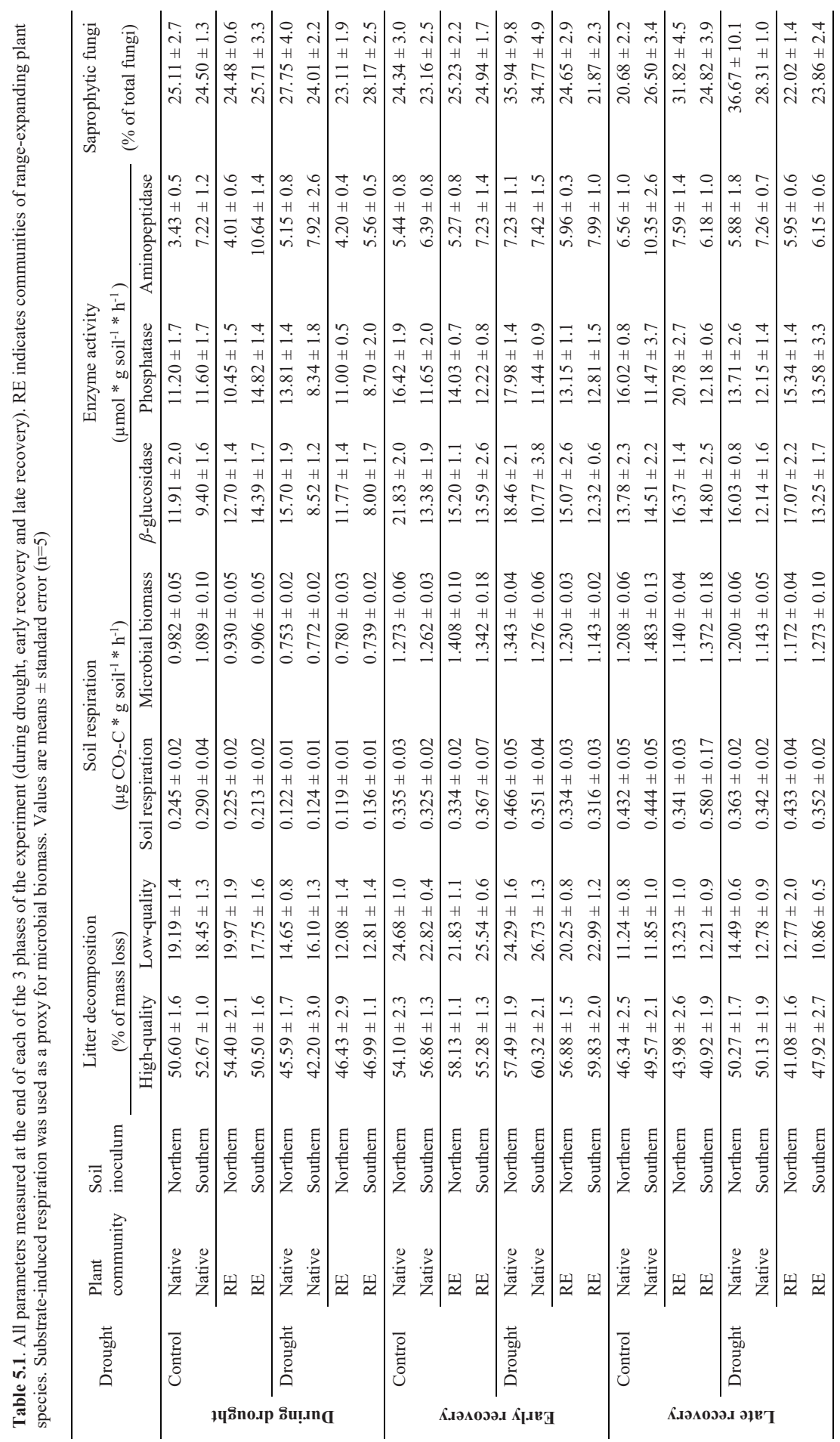




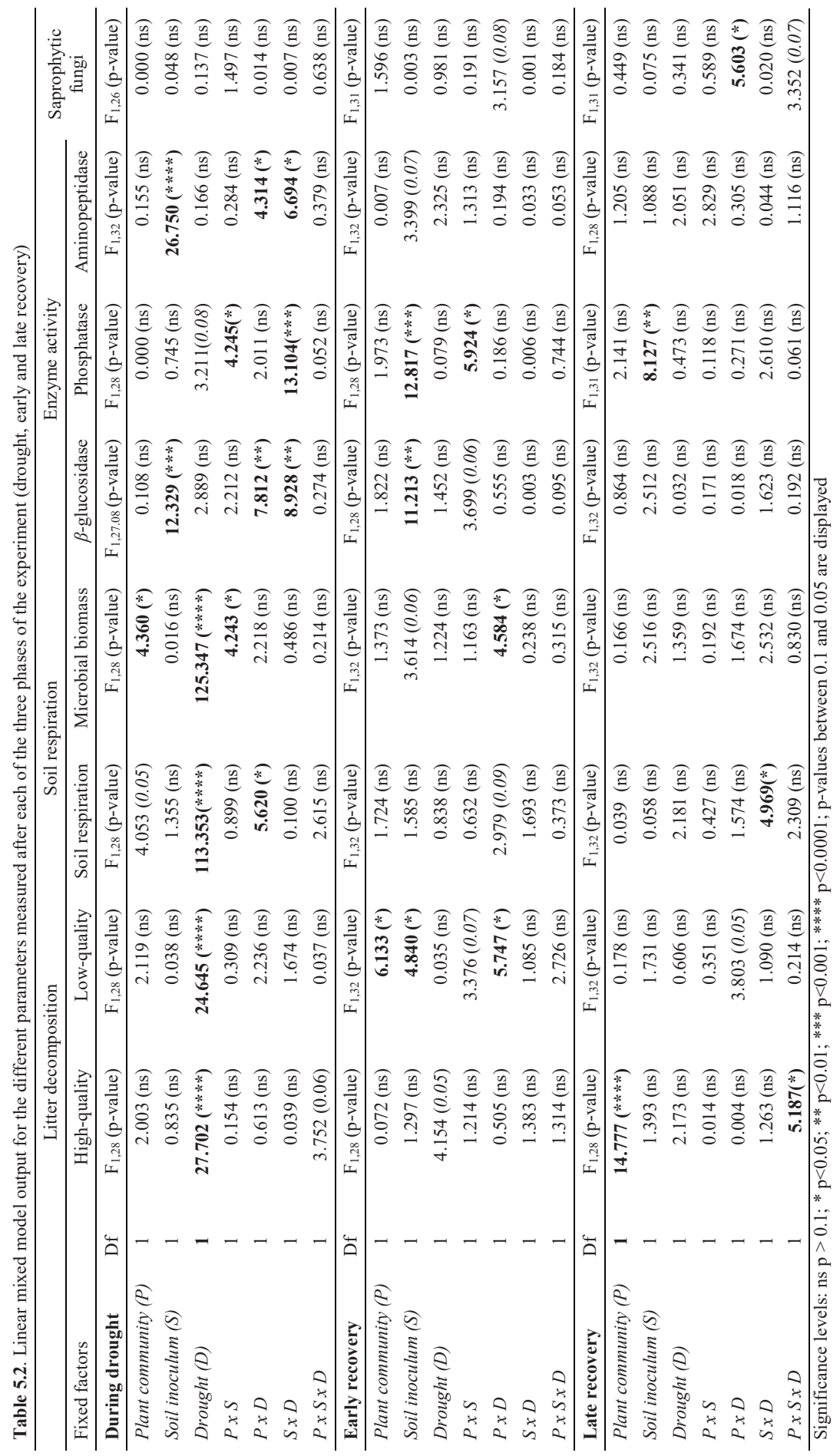


Mass loss of low-quality litter was decreased by $26.2 \%$ on average across all treatments with respect to the control during drought (Fig. 5.1D), but there was no influence of plant community origin and soil inoculum (Table 5.3). During both recovery phases, plant community origin significantly affected the proportional change from the control regardless of soil inoculum. Mass loss of low-quality litter was more positively affected by drought history in soils of native plant communities than in soils of range expanders (Fig. 5.1E, 5.1F, Table 5.3). There was no effect of soil inoculum on the change in litter mass loss during the recovery phases.
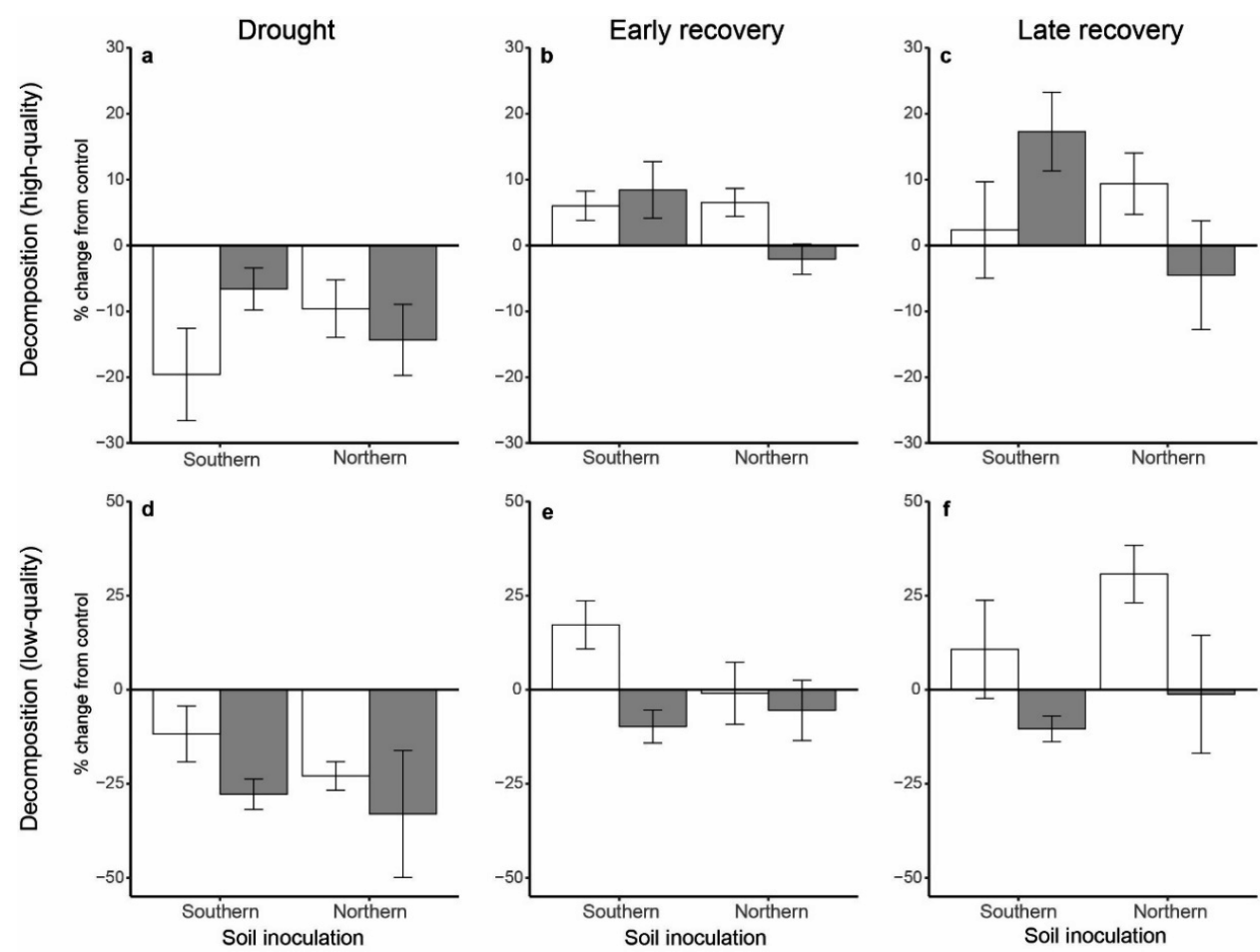

Fig. 5.1. Proportional change from the control in mass loss of high-quality substrate $(A, B, C)$ and low-quality substrate $(\mathrm{D}, \mathrm{E}, \mathrm{F})$ during each of the 6-week experimental phases: drought $(\mathrm{A}, \mathrm{D})$, early recovery $(\mathrm{B}, \mathrm{E})$ and late recovery $(\mathrm{C}, \mathrm{F})$ after drought. Means with standard error $(\mathrm{n}=5)$ are presented for native (white bars) and range-expanding (grey bars) plant communities. 


\section{$\underline{\text { Soil basal respiration and soil microbial biomass (SIR) }}$}

In line with litter mass loss, drought decreased soil basal respiration and microbial biomass in all soils (Table 5.1, Table 5.2). Soil respiration was higher beneath native plant communities than beneath range-expanders under control conditions, but this effect was reversed under drought conditions (Table 5.1, Table 5.2). Soils of range expanders had higher microbial biomass in southern soils than in northern soils and, overall, microbial biomass was higher beneath native plant communities compared to range expanders at the end of the dry period. In the early recovery, there were no effects of our treatments on soil respiration. However, there was a significant interaction of plant community origin and drought history for microbial biomass (Table 5.2), indicating that history of drought still had a negative effect on microbial biomass in soils of range expanders but not in soils of natives. In the late recovery, there was an interaction of soil inoculum and drought history on soil respiration (Table 5.2). Southern soils with a history of drought had lower respiration rates than controls, but this did not occur for northern soils (Table 5.1). However, there were no effects of our treatments on soil microbial biomass in the late recovery.

Right after the drought period, the proportional change from the control in both soil respiration and microbial biomass was more negative in soils of native plant communities than in soils of range-expanding plant communities (Fig. 5.2A, 5.2D, Table 5.3), indicating that soils of range-expanding plant communities are less hampered by drought than soils of native. During the early recovery, microbial biomass was more negatively affected by drought in soils of range-expanding plant communities than in soils of natives (Fig. 5.2B, 5.2E, Table 5.3). Although it was the same trend, this effect was not significant for soil respiration (Table 5.3). In the late recovery, the proportional change from the control in soil respiration tended to be negative for southern soils and positive for northern soils (Fig $5.2 \mathrm{C}, 5.2 \mathrm{~F}$, Table 5.3). 

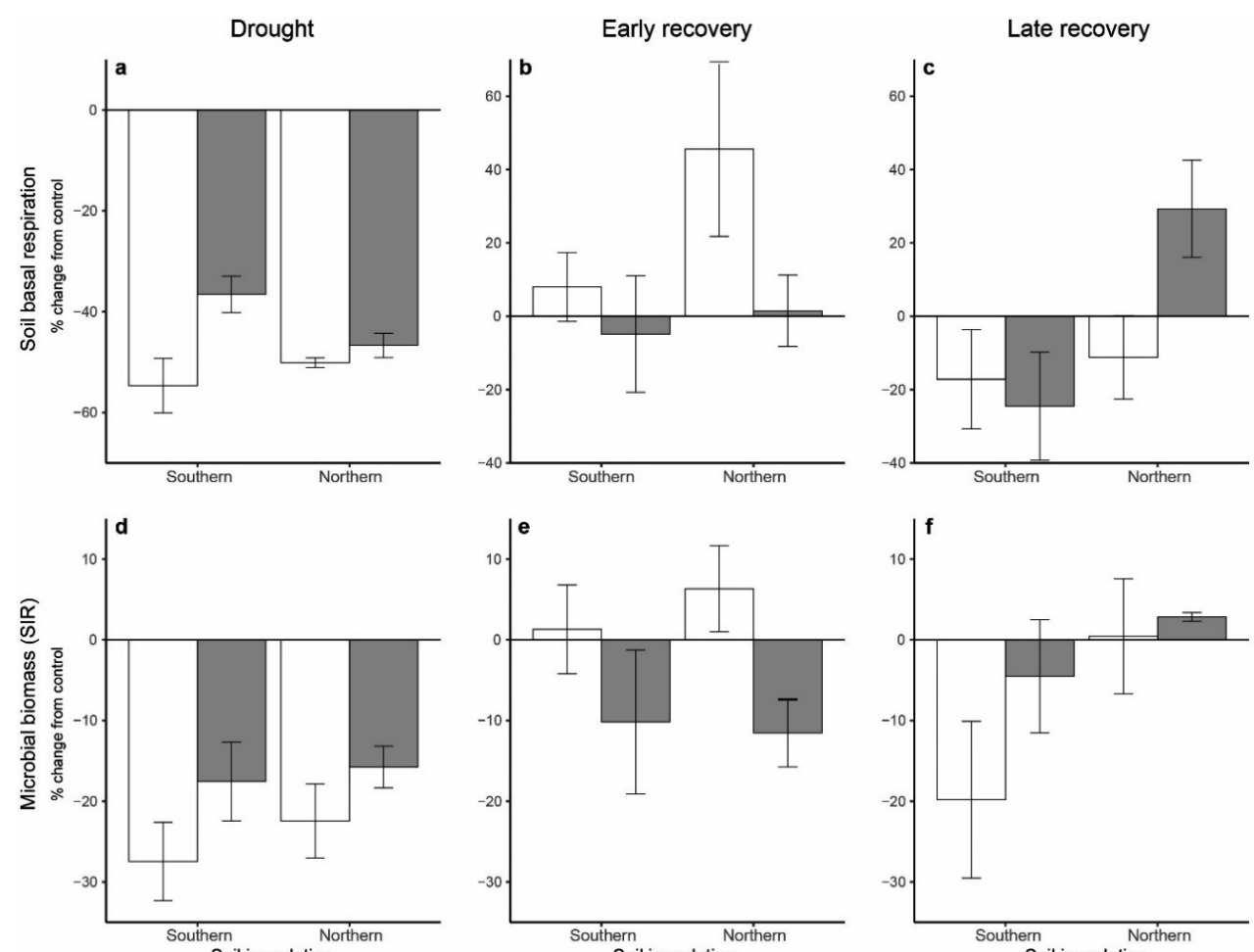

Fig. 5.2. Proportional change from the control in soil basal respiration $(A, B, C)$ and microbial biomass $(\mathrm{D}, \mathrm{E}, \mathrm{F})$ at the end of the 6-week experimental phases: drought $(\mathrm{A}, \mathrm{D})$, early recovery $(\mathrm{B}, \mathrm{E})$ and late recovery $(\mathrm{C}, \mathrm{F})$ after drought. Means with standard error $(n=5)$ are presented for native (white bars) and range-expanding (grey bars) plant communities.

\section{Enzyme activity stability}

At the end of the drought period, the interaction between soil inoculum and drought affected the potential enzyme activity rates for all three enzymes. Drought stimulated glucosidase and phosphatase activity in northern soils but had decreased their activity in southern soils, and vice-versa for aminopeptidase activity (Table 5.1, Table 5.2). Drought also stimulated glucosidase activity beneath native plant communities but decreased it under range expanders. History of drought did not affect enzyme activity upon rewetting of the soil in both early and late recovery measurements. Glucosidase and phosphatase activity were higher in northern soils compared to southern soils during the recovery, but there was no significant effect of soil inoculum on aminopeptidase activity (Table 5.2). 
In soils collected at the end of the drought period, the proportional change from the control was more positive in northern soils compared to southern soils for all three enzyme activities, indicating the positive effect of drought on enzyme activity in northern soil and a negative effect on southern soils (Fig. 5.3A, Table 5.3). During both early- and late recovery phases, there were no significant effects of plant community origin or soil inoculum on the proportional change from the control (Fig. 5.3B, 5.3C, Table 5.3).
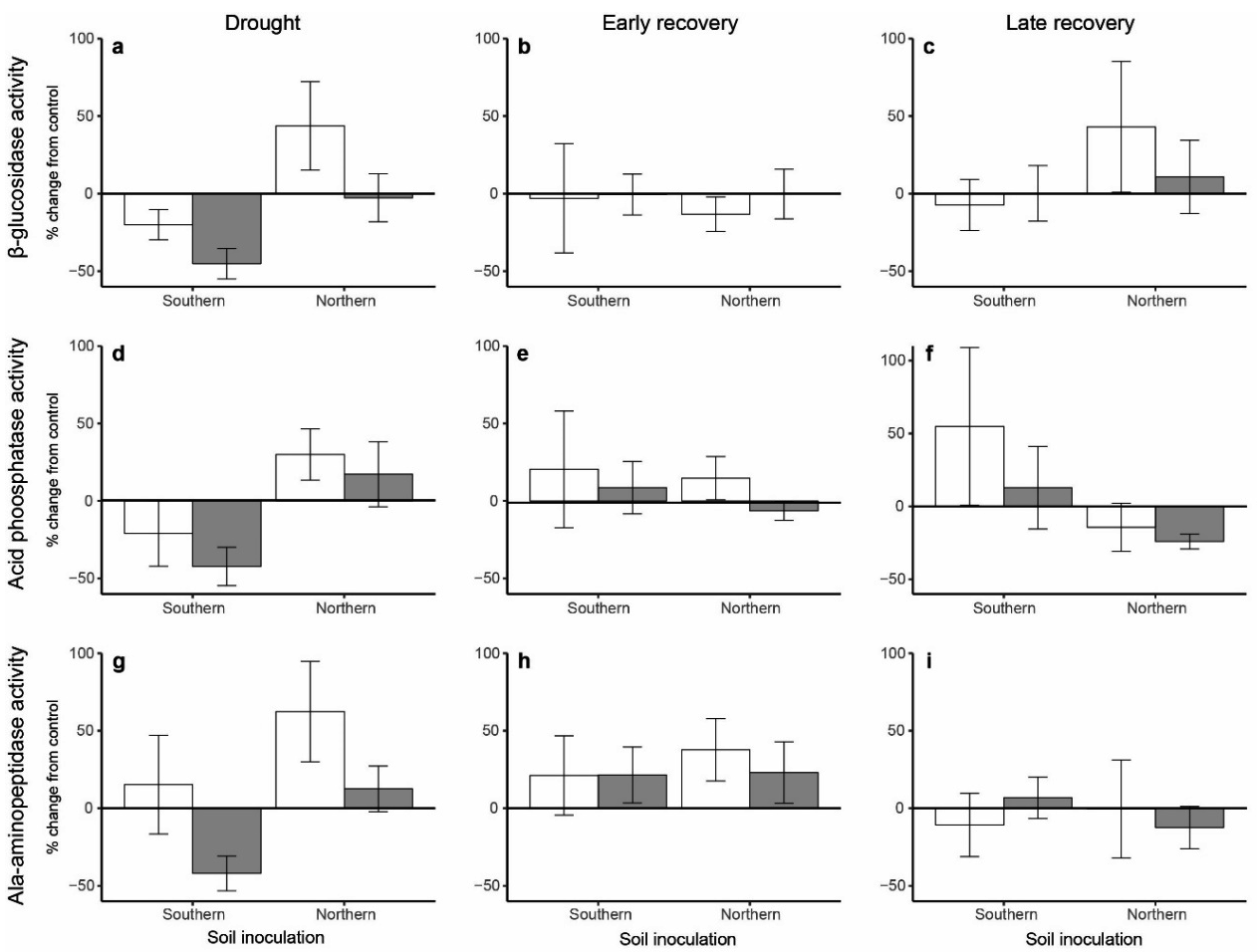

Fig. 5.3. Proportional change from the control in b-glucosidase $(A, B, C)$, acid phosphatase $(D, E, F)$ and alanine aminopeptidase $(\mathrm{G}, \mathrm{H}, \mathrm{I})$ activities at the end of the 6-week experimental phases: drought $(\mathrm{A}, \mathrm{D}, \mathrm{G})$, early recovery $(\mathrm{B}, \mathrm{E}, \mathrm{H})$ and late recovery $(\mathrm{C}, \mathrm{F}, \mathrm{I})$ after drought. Means with standard error $(n=5)$ are presented for native (white bars) and range-expanding (grey bars) plant communities. 


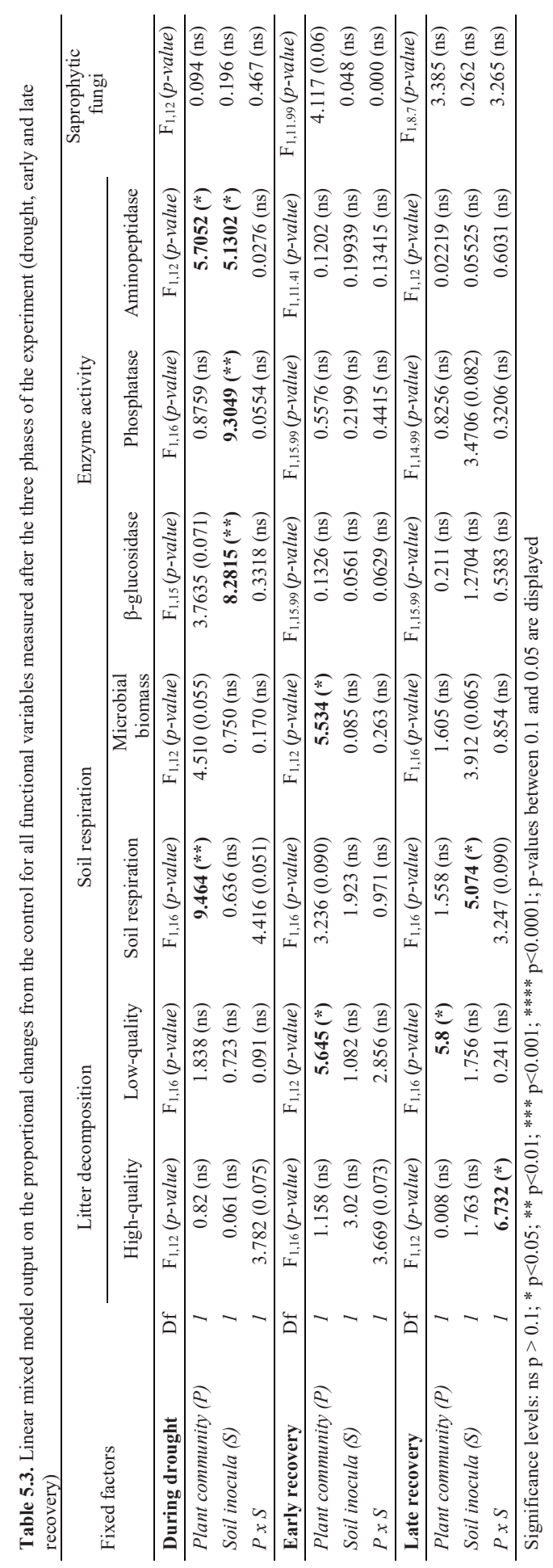




\section{Soil saprophytic fungi}

There were no differences in the relative abundance of saprophytic fungi between drought and control and between plant communities and soil inoculum from different origins at the end of the drought period (Table 5.1, Table 5.2). During early recovery, the relative abundance of soil saprophytic fungi tended to be higher in soils of native plant communities with a drought history compared to the control, but there was no effect of drought history for soils of range-expanding communities (Table 5.1, Table 5.2). This effect was stronger during late recovery as was indicated by a significant plant community $x$ drought interaction (Table 5.2). The proportional change from the control indicates the same trend; saprophytic fungi in soils with drought were, indeed, stimulated with respect to the control after rewetting of the soil, but only in soils of native plant communities and not in soils of range-expanders (Fig. 5.4, Table 5.3).
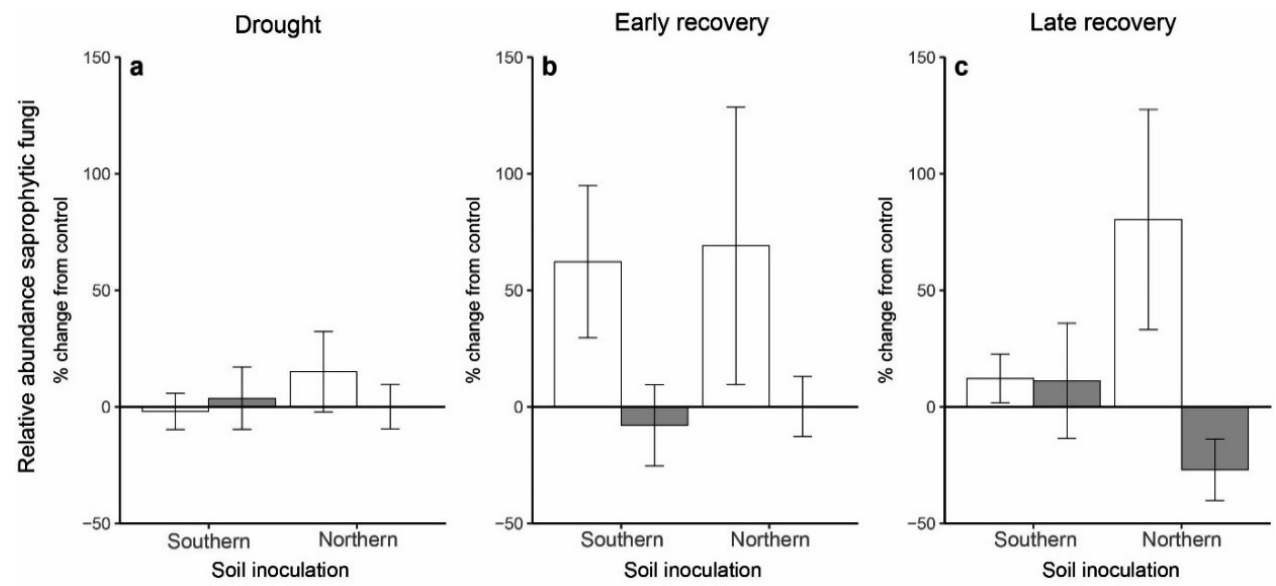

Fig. 5.4. Proportional change from the control in the relative abundance of soil saprophytic fungi at the end of the 6-week experimental phases: drought (A), early recovery (B) and late recovery (C) after drought. Means with standard error $(n=5)$ are presented for native (white bars) and rangeexpanding (grey bars) plant communities.

\section{Discussion}

Multiple global change factors, such as species range shifts and extreme weather events, may affect species composition in terrestrial ecosystems as well as important soil processes, such as litter decomposition and nutrient cycling. In order to predict how ecosystems will respond to global change, it is important to study both independent and 
interactive effects of the different factors of global change. Here, we used a factorial experiment to test the hypotheses that soils of range-expanding plants originating from Southern Europe are more resistant and resilient to drought than soils of native plant communities, especially when range-expanding plants grow in soils with southern soil biota. Our results show that plant community origin and soil inoculum can modify the magnitude and direction of the responses of soil functions to extreme drought. In general, responses were strongest shortly after rewetting and often associated with a positive effect of drought history on soil activity beneath native plant communities. However, these responses differed between specific soil functions measured and varied with the different experimental phases.

\section{Responses to drought}

In line with our first hypothesis, we found that soil respiration was less hampered by drought in soils from range-expanders than in soils from native plant communities. Under moderate drought, plant species may increase root exudation, and thereby maintain the activity of the soil microbial community in the root zone (Palta and Gregory 1997, Preece and Peñuelas 2016, Birgander et al. 2017, Ahmed et al. 2018). However, we do not know whether plant origin in our study (native vs. range expander) resulted in different carbon inputs to the soil under drought. In contrast with our first hypothesis, plant community origin did not influence the resistance to drought for litter decomposition, microbial biomass and the abundance of saprophytic fungi. Instead, in our study the severe summer drought may have reduced soil community activity in all treatments as a result of low substrate diffusion hampered by low soil water availability (Stark and Firestone 1995, Manzoni et al. 2012).

In contrast to our expectation that soils with an inoculum from Southern Europe would be more resistant to drought than soils with an inoculum from Northern Europe (Hawkes et al. 2017), we found that northern and southern soils generally responded to drought in the same way. This means that within the latitudinal gradient selected we found no evidence that differences in latitudinal origin of the soil influences current responses of soil community functioning to drought. Only for the response of enzyme activity we found that soil inoculum played an important role. Interestingly, the interaction effect of soil inoculum and drought was dependent on the specific enzyme activity measured (e.g. phosphatase and glucosidase activity were enhanced by drought in northern soils, but decreased in southern soils). Even though the soil inocula only represented $30 \%$ of the 
topsoil in our mesocosms, differences in the soil mineral fraction between soil inoculum types may have influenced the stabilization of extracellular enzymes during periods of low water availability (Stursova and Sinsabaugh 2008) and hence may explain differences in enzyme activities under drought.

Responses to soil rewetting after drought

Our second hypothesis was that soil functions would recover faster from drought (i.e. higher resilience) in soils of range expanders, especially in combination with southern soil communities. However, we found rewetting to immediately stimulate decomposition of low-quality substrate (i.e. rooibos tea), microbial biomass and relative abundance of saprophytic fungi in soils of native plant communities, independent of soil inoculum. Soils of range-expanding plants appeared to be more conservative in their response to drought. As a result, decomposition activity recovered more gradually and rewetting did not influence microbial biomass and the relative abundance of saprophytic fungi in comparison to the control.

It is well established that rewetting of dry soil generally enhances soil microbial activity (Birch 1958). This so-called 'Birch' effect seems to be caused by metabolic adjustments at the level of individual microbial cells, which immobilize solutes intracellularly during drought and release them upon rewetting thereby stimulating microbial activity (Fierer et al. 2003, Schimel et al. 2007). Our results show that native plants enhanced the Birch effect in terms of decomposition (low-quality substrate) and microbial growth as compared to range expanders. Previous work has explained differences in the Birch effect between plant communities via altered soil microbial communities (Fierer and Schimel 2002). Our findings that microbial biomass, as well as the relative abundance of saprophytic fungi increased under native communities, suggests that plant-induced changes in the microbial community may indeed explain differences in the Birch effect between native and rangeexpanding plant communities. These plant-induced effects may operate, for example, via drought-induced root mortality and turnover or via different patterns of root exudation upon rewetting. Such effects have not yet been shown for range-expanding plant species.

In our study, we did not find an effect of drought history on soil respiration upon rewetting. Studies investigating responses of soil respiration after rewetting often focus on a time frame of hours to days after rewetting of the soil (Göransson et al. 2013, Meisner et al. 2017). In contrast, we assessed the first responses to rewetting after six weeks, when immediate changes in soil respiration may have recovered already, while the impact of 
drought history on longer-term processes, such as litter decomposition and changes in microbial biomass, are still present. Therefore, responses of plants and soil communities to rewetting after drought may cascade from altered respiration rates, which occur immediately upon rewetting (Meisner et al. 2013a), to promoting changes on soil (saprophytic) microbial communities, decomposition and nutrient dynamics that persist to later stages after the drought stress (Fierer and Schimel 2002, Fierer et al. 2003).

\section{Plant response strategies}

Our results that short-term responses of soil functions to rewetting were dependent on plant community origin and soil inoculum indicate that plant range shifts can play a role in ecosystem responses to global change (Kardol et al. 2010). Even though climatic parameters can directly drive changes in soil communities and their functions, it has been shown that plants can modulate these effects via different mechanisms such as changes in soil microclimate, plant-soil interactions and the provisioning of carbon resources (Dukes and Hungate 2002, Waldrop and Firestone 2006b, Drigo et al. 2007).

While the presence of native plant communities stimulated soil activity as a response to soil rewetting after drought, the presence of range-expanding plants decreased the magnitude of the soil functioning responses to drought. Instead, soil functions beneath range-expanding plants recovered to control levels without surpassing them, suggesting a more conservative response upon soil rewetting. This conservative response of soil functioning promoted by range-expanding plants could be the result of adaptation to drought events, typically more incident in South Europe where range-expanding plants originate from. In spite of the relatively limited nature of our experiment (i.e. single drought event, single year, short time frame), these results may add to the discussion on whether range-expanding plants may increase the stability of soil ecosystem functioning, as suggested by our data. In contrast to the effects of plant community origin, we found less impact of the origin of the soil inoculum on responses of ecosystem functions to drought, suggesting functional redundancy of soil communities from these different latitudes when compared under the same environmental conditions (Wertz et al. 2007).

Both the duration of drought periods and the frequency of drying-rewetting cycles can have important consequences for soil ecosystem functioning (Fierer and Schimel 2002, Meisner et al. 2013a). Short-term dynamics of soil functions upon rewetting may become most relevant under a future climate scenario with recurrent drying and rewetting. Under such scenarios depending on seasonality, plant range expansion may have the potential to 
substantially influence ecosystem-level processes over longer time scales, such as net ecosystem productivity or soil carbon balance. Furthermore, as soil activity responses to dry-rewetting cycles can have a substantial contribution to global $\mathrm{CO}_{2}$ dynamics, especially in water-limited ecosystems (Almagro et al. 2009, Matteucci et al. 2015), we emphasize the need to also include the effect of changes in plant communities (e.g. as results of climate warming-induced range shifts) on soil activity responses. To understand the full potential of plant range expansion to modify soil ecosystem functioning in the longer term, it will be essential to study their interactive effects with future climate scenarios and to get a mechanistic understanding of these interactions.

\section{Conclusion}

We conclude that plant range expansion may influence short-term responses of soil ecosystem processes to rewetting after drought periods, and that these effects are independent of the geographical origin of the soil community. In particular, rangeexpanding plant species from warm climate zones affected soil functioning in a more conservative manner following soil rewetting than native plant species. We propose that these different responses could have ecosystem level consequences depending on the nature and recurrence of the drought and on whether drought occurs during peak growing time for the plants or later in the season.

\section{Acknowledgements}

We thank Rosanne van der Linden for her practical assistance. We thank Emilia Hannula for providing the Funguild data, and Basten Snoek and Mattias de Hollander for bioinformatics support. The authors would also like to thank everybody who helped setting up and maintaining the mesocosm experiment over the last years. This work was funded by the ERC-Advanced grant SPECIALS (260-55290) to Wim H. van der Putten. Ciska Veen was supported by a NWO-VENI grant (863.14.013). 


\section{Supplementary information}

Table S5.1. Native and range-expanding plant species used during the mesocosms since its establishment in 2013. During the first 2 years of experiment, 3 range-expanding species and 3 related natives were planted in combination with 3 native grass species $(*)$ to condition the soil. During 2015 and 2016, we planted the experimental plant communities. In 2016, the drought experiment was carried out.

\begin{tabular}{|c|c|c|}
\hline $\begin{array}{l}\text { Plant community } \\
\text { origin }\end{array}$ & $2013-2014$ & $2015-2016$ \\
\hline \multirow{8}{*}{ Native } & Centaurea jacea L. & Centaurea jacea L. \\
\hline & Tragopogon pratensis L. & Geranium molle L. \\
\hline & Angelica sylvestris L. & Tragopogon pratensis $\mathrm{L}$. \\
\hline & & Rorippa sylvestris (L.) \\
\hline & Phleum pratense L. * & Sinapis arvensis L. \\
\hline & Poa trivialis L. * & Crepis biennis L. \\
\hline & Festuca rubra L. * & Pulicaria dysenterica L. (Bernh.) \\
\hline & & Alliaria petiolata (M. Bieb.) \\
\hline \multirow{8}{*}{ Range expanding } & Centaurea stoebe L. & Centaurea stoebe $\mathrm{L}$. \\
\hline & Tragopogon dubius (Scop.) & Geranium pyrenaicum (Brum. F.) \\
\hline & Angelica archangelica L. & Tragopogon dubius (Scop.) \\
\hline & & Rorippa austriaca (Crantz) Besser \\
\hline & Phleum pratense L. * & Rapistrum rugosum (L.) All. \\
\hline & Poa trivialis L. * & Lactuca serriola $\mathrm{L}$. \\
\hline & Festuca rubra L. * & Dittrichia graveolens (L.) \\
\hline & & Bunias orientalis $\mathrm{L}$. \\
\hline $\begin{array}{l}\mathrm{N}^{0} \text { individuals per } \\
\text { species }\end{array}$ & 6 & 8 \\
\hline Total number of plants & 36 & 64 \\
\hline
\end{tabular}



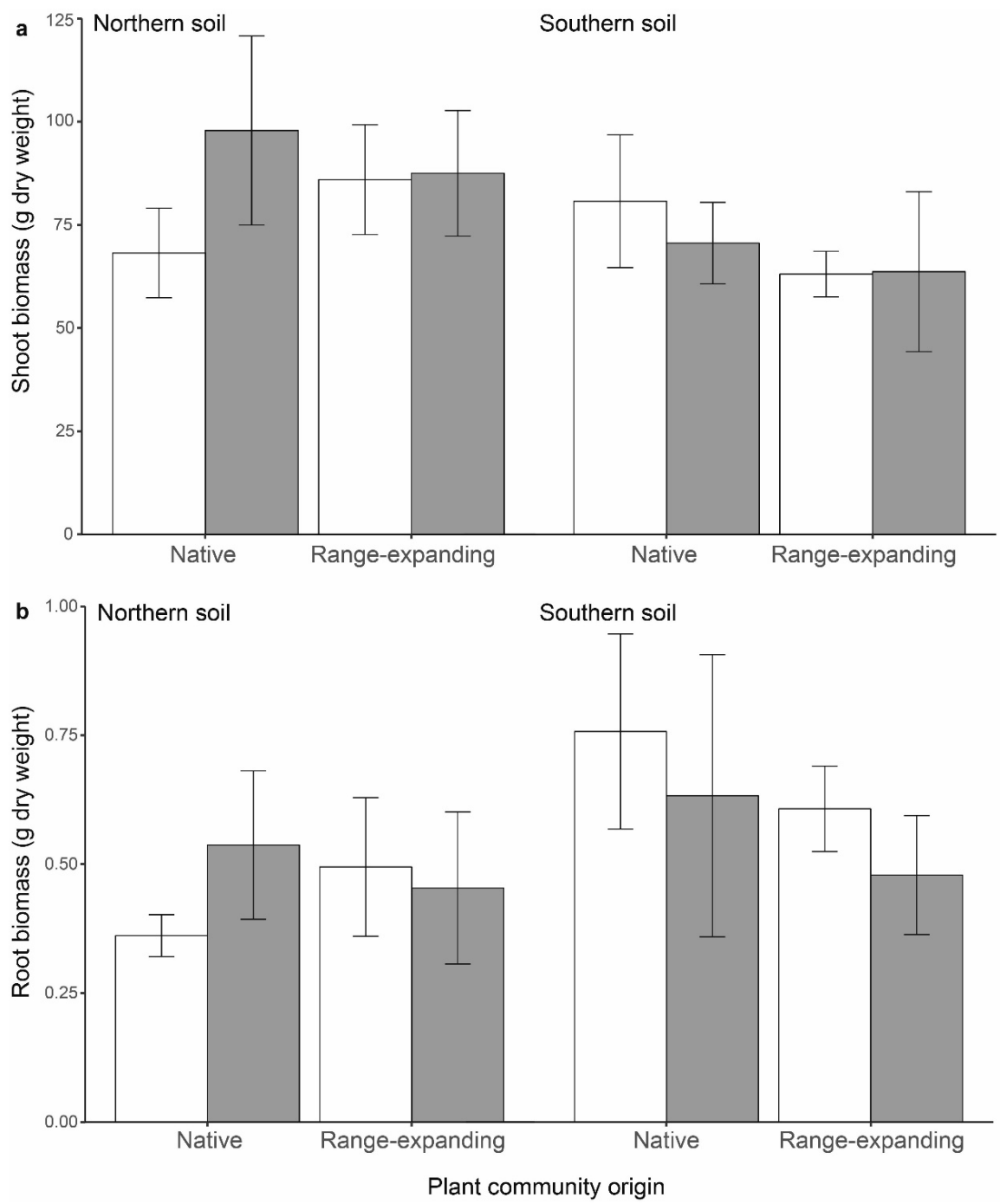

Fig. S5.1. Shoot and root biomass at the end of the growing season in the watered control (white bars) and in the drought treatment (grey bars). Bars indicate means with standard error $(n=5)$. 


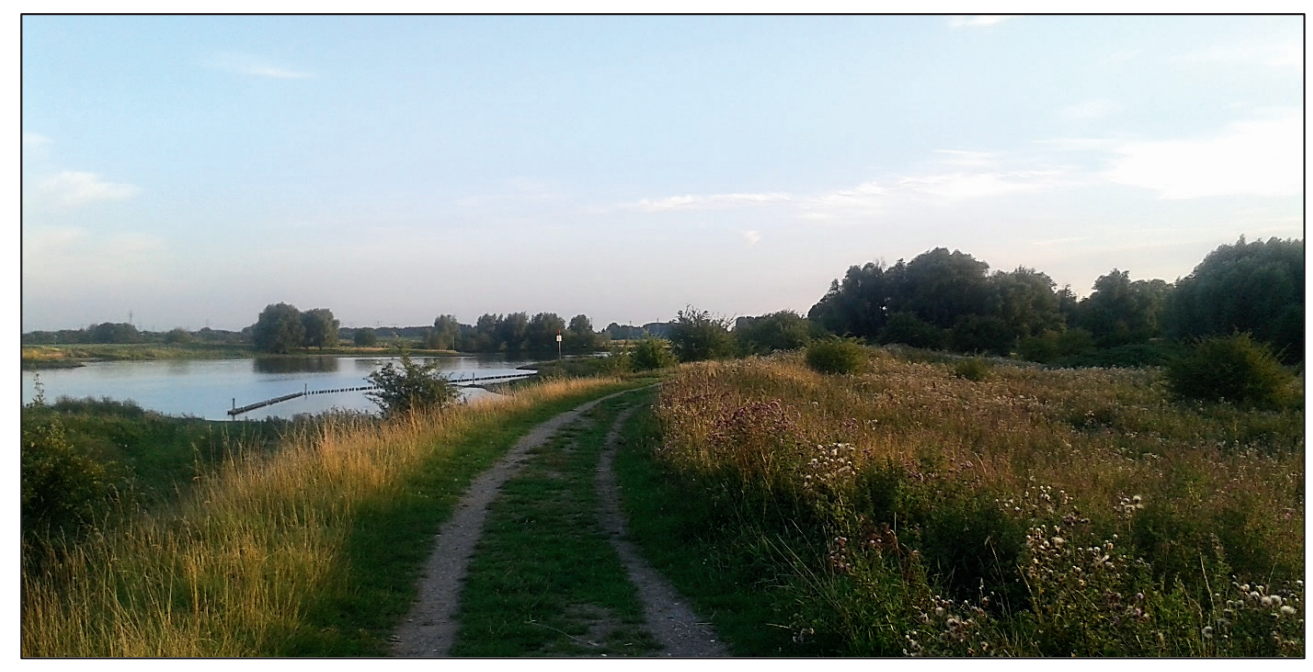




\section{Chapter 6}

General discussion 
The general aim of this thesis was to investigate indirect plant-soil interactions of rangeexpanding plant species, which operate via the plant inputs to the soil, and the conversion of these inputs by the soil decomposer community into plant-available nutrients. I was especially interested in how these plant-soil interactions of range-expanding plants compare to those of natives in order to determine functional consequences of range expansion for the invaded ecosystems. I investigated whether plants that expand their range within continents would resemble invasive exotic species in terms of the chemistry and nutrient concentration in their leaves and in the soils where they grow, and I compared range expanders with native plant species (Chapter 2). Then, in a controlled greenhouse experiment, I determined the role of plant origin on bacterial community composition in the rhizosphere and the functional capacity of the soil community (Chapter 3). In Chapter 4, I investigated plant-soil feedback effects driven by plant litter and living plants and determined effects of this soil conditioning on plant biomass and litter mass loss. Finally, I investigated how plant community changes resulting from range expansions can interact with extreme drought events to modify soil processes such as litter decomposition and nutrient cycling (Chapter 5). In the present chapter, I discuss the major findings of this thesis in the context of published literature on both range-expanding and exotic plant species. To finalize, I provide some ideas for further research and conclude with the main outcomes of this thesis research.

\section{Predictability in plant range expansion}

A main aim of invasion ecology is to be able to predict when and where exotic plant species may become established and become disproportionally abundant (Hayes and Barry 2008). To be able to make such predictions, research has focused on determining widespread characteristics associated with exotic plant invasions. For example, plant traits such as growth rate, fitness or biomass allocation to shoots are often higher in invasive plant species than in non-invasive species (van Kleunen et al. 2010). Furthermore, microbial community structure in the soil can differ between invaded and non-invaded sites (Kourtev et al. 2002a). Besides, soil nutrient content and plant primary chemistry have also been associated to plant invasiveness because exotic species most often have higher nutrient levels than co-occurring natives (Pyšek et al. 2012, Sardans et al. 2017). These differences in soil nutrient content and plant chemistry may further promote invasive 
abundance of exotic plant species, as exotics have been shown to benefit more than natives do from high soil nutrient availability (Blumenthal et al. 2009, Liu and van Kleunen 2017).

In my research, I aimed at determining whether these characteristics of exotic plant species may also be present in range-expanding plant species. The results of Chapter 2 indicate that, based on the species selected, there is no general pattern of plant and soil nutrients in relation to plant range expansion. I mainly observed species-specific effects, where the range-expanding species in some cases and the native species in other cases had higher nutrient concentration in leave tissues and in the soils. Therefore, the main outcome from this study is that even though some range-expanding plant species may follow such patterns of invasive species, these characteristics cannot be associated to non-native plant origin in general. Then in Chapter 3, I examined soil bacterial communities of sites where both natives and range-expanding plant species grow in the field. There, I show that plant species are associated to different bacterial communities in the soil. Besides soil bacteria, I also show that there were differences in soil $\mathrm{pH}$ and nutrient content between plant species. However, there was not a general pattern of plant origin for neither soil communities nor abiotic characteristics. This is line with the results from Chapter 2, and altogether my research shows that range-expanding plant species may establish in sites that differ from their original range and from sites of related native plant species. Yet, when present, these differences are species-specific and cannot be attributed to differences in their geographical origin in general.

Despite the overall patterns in nutrients associated to exotic invasive species, there is substantial variation between individual studies (Vilà et al. 2011, Pyšek et al. 2012). Exotic plant species seem to display higher plasticity than natives (Funk 2008), perhaps making them more capable of occupying sites with varying abiotic conditions. Certainly, variation is also high among the limited number of range-expanding plant species studied here. Increasing the number of replicate range-expanding plant genera is likely to increase the capacity to generalize about the role of plant origin in plant range expansions and improve predictions of what species are likely to expand their range and what site characteristics are most common for range expanding plant species to become established. 


\section{Functional consequences of plant range-expansion on native ecosystems}

A key question to understand the ecological consequences of range expansion is to what extent range-expanding plant species may alter soil functions and biogeochemical cycles in the new range. Determining the impacts of exotic plant species on ecosystems has been a focus of plant invasion studies (Levine et al. 2003, Vilà et al. 2011). Exotic plant invasions have often been associated to increases in nitrogen pools and cycling in the soil (Allison and Vitousek 2004, Ashton et al. 2005, Hawkes et al. 2005, Castro-Díez and Alonso 2017), soil enzymatic activity (Kourtev et al. 2002b, Allison et al. 2006) and to faster litter decomposition (Rothstein et al. 2004, Ashton et al. 2005, Arthur et al. 2012, Zhang et al. 2016a). These changes in important soil functions can have local consequences for native ecosystems and can enhance the performance of exotic plant species over natives via competition and soil legacy effects (Ehrenfeld et al. 2005).

The results of this thesis suggest that there are no overall impacts of range-expanding plant species on soil processes such as litter decomposition and soil enzyme activity. Instead, I show that soil microbial communities of range-expanders and related natives share similar ability to utilize organic substrates of varying recalcitrance after conditioning via the rhizosphere (Chapter 3 and 4), but also after conditioning with plant litter and rhizosphere plus plant litter (Chapter 4). Furthermore, soils of range-expanders and related natives display similar ability to breakdown plant litter, especially of aboveground plant litter (Chapter 4), and show similar decomposition rates under non-drought conditions (Chapter 5). However, this does not mean that there are no differences between plant species or that the impact of range-expanding and native plants is similar under all environmental conditions. First, in Chapter 4 I have shown that litter mass loss is not related to plant origin but to plant identity. There I show that in some species-specific cases, such as in genera Centaurea or Rorippa, litter of range-expanders may indeed have higher decomposition rates. Yet, this is not the case for the other two plant genera used in the experiment, so that this effect does not seem to be caused by their different origin, but rather to specific differences in their chemistry (Macel et al. 2014, Wilschut et al. 2017). Secondly, in the mesocosm drought experiment of Chapter 5, I showed that soils of native plant communities that were subjected to drought stimulated soil functioning upon rewetting of the soil, whereas this was not observed in soils of range-expanders. These short-term responses may be due to adaptation of the range expanders under climate conditions that include long summer droughts, such as occurring in Mediterranean and 
East-Continental Europe. In such areas, interactions between plants and soils may favour nutrient retention after drought and rewetting cycles. At the longer term, however, these effects were not detectable anymore. Although the effects of plant origin in this experiment were limited in terms of the time they lasted, changes in the short-term responses to drought events may become more relevant in scenarios of recurrent drought-rewetting cycles. Under these scenarios of recurrent climate extremes, soils under communities that contain range-expanding plant species may function differently, possibly less responsive to a set-back of normal rainfall conditions, than those composed of native species only.

\section{The role of specialized decomposer communities}

Decomposer communities in the soil may specialize to decompose litter from the locally dominant plant species (Gholz et al. 2000, Ayres et al. 2009a, Ayres et al. 2009b). This specialization may have developed in the same co-evolutionary manner as plants and their specialized enemies have co-evolved. The enemy release hypothesis predicts that exotic plant species outside their native range may benefit from the lack of co-evolved specialist enemies (Keane and Crawley 2002). Based on the same idea and provided that plant species have co-evolved specialist decomposer communities in their native range, exotic plant species that establish outside their native range may lack specialized decomposer communities. This mismatch between plants and their specialized decomposer communities in the new range may have consequences for decomposition, nutrient cycling and thereby plant growth. In this thesis, I examined the role of specialized decomposer communities in plant range expansion using two different approaches.

During the feedback phase on litter decomposition of Chapter 4, I tested the role of specialized decomposer communities in plant range expansion. There, I used the soils conditioned by plant litter to determine how the identity of the conditioning litter species affected contemporary decomposition of the same species or of the related native or rangeexpander species. I showed that litter mass loss was mainly controlled by litter species identity (Cornwell et al. 2008), and that the previous litter used to condition the soil did not influence contemporary mass loss. Therefore, I could not find evidence for the loss of specialized decomposer activity. Alternatively, paired range-expander and native plant species may share specialist decomposer communities as they are expected to differ in origin only, while traits are relatively similar. Thus, I could not observe a benefit from 
specialist decomposers between phylogenetically related species, while studies using plant species with contrasting litter quality can identify specialization in decomposer communities (Freschet et al. 2013, Veen et al. 2015a).

Interestingly, for root litter, mass loss of range-expander roots was greatest in soils conditioned by the litter of native plants. This effect was mainly observed in Centaurea and suggested that, in some cases, soils of native plants may further increase root decomposition of range-expanding plant litter in the new range. In general, the contribution of plant roots on decomposition activity in the soil and plant-soil feedback has been overlooked in the literature (Ma et al. 2016, Zhang et al. 2016b). Yet, a recent study has shown that the presence of local roots may not only stimulate decomposition but also mediate specific litter-decomposer interactions (Tian et al. 2018). Thus, more research would be needed to investigate to what extent there are differences in the role of specialized decomposers for decomposition of above and belowground litter, as root decomposability is expected to differ substantially from shoot litter (Birouste et al. 2012, Freschet et al. 2013), and to determine how dominant plant species may influence the role of specialized decomposers.

In Chapter 5, I had expected that soil functions under range-expanding plant communities would be less affected by drought stress when soils from the expansion range were inoculated with soil from the original range, because these plants and soils have coevolved under dry weather conditions. My results, however, were not in support of this hypothesis because there was no significant interaction effect of plant community (rangeexpander or native) and soil (from new or original range) on soil functions during or after drought. Previously, Hawkes et al. (2017) have shown that the respiration of soils from drier areas is less sensitive to moisture changes than soils from wetter areas. In my experiment, independently of plant community identity, litter mass loss after rewetting was higher in soils from the original range than in soils from the expansion range. In addition, two out of three enzyme activities measured were higher in soils from the expansion range throughout the experiment as well. This indicates that while soils from the original and expansion range may differ in the response to drought for specific functions as also shown by Hawkes et al. (2017). However, these responses of soils from the original and new range do not seem to interact with the origin of the standing plant community in my experiment. 
The results obtained in my thesis suggest a limited role of specialized interactions between (microbial) decomposer communities and plant species in the context of range expansions. My conclusions, however, need to be considered within the framework of my experimental approach. First of all, effects of specialist decomposers may develop and become stronger over time, and the limited length of my experiments (circa 3 months) perhaps has limited the effect strengths that I observed. Time has been an important factor for the development of negative interactions of exotic plants and specialized enemies in the new range (Diez et al. 2010). In that study, negative plant-soil feedbacks developed over periods of decades to centuries. Secondly, decomposers are suggested to be generalists and as a result, the degree of specialization may only be sensitive to higher taxonomical levels of plants or larger functional differences between plant species (Veen et al. 2015a). Consequently, when comparing congeneric species there may be limited scope for finding impacts of specialized decomposer communities (but see Madritch and Lindroth (2011)).

\section{Future scenarios of global change and the role of plant range expansions}

Extreme weather events (drought or extreme rainfall episodes and more recurrent heat waves) are predicted to increase in areas where range-expanding plant species originating from South Europe are establishing, such as in the Netherlands (KNMI 2015, NDFF 2018). Consequently, plant range expansion interacts with other, more direct, effects of climate change in the expansion range. In Chapter 5, I show that there are little differences in the effect of range-expanding plant communities on soil functioning as compared to native plant communities. Yet, after extreme drought and shortly upon rewetting of the soil, I show that range-expanding plant communities stimulate litter decomposition activity in the soil to a larger extent than native plant communities. Thereby, my results suggest that the presence of range-expanding plant species or natives may become more relevant under scenarios of extreme weather events, which will become more regular under current climate warming. Furthermore, I show that beyond the interactions with the standing plant community, drought also has a general and strong effect on basal soil respiration and on litter breakdown, which is in line with previous climate manipulation and gradient studies (Garten et al. 2009, Schindlbacher et al. 2012, Liu et al. 2018). My research also supports the idea that climate change is currently affecting terrestrial ecosystems in multiple direct and indirect ways. Thus, in order to fully understand how ecosystems may 
respond to climate change, it will be crucial to investigate both direct and indirect effects via changes on plant community composition on soil communities and their functioning (Bardgett et al. 2008, Bardgett and van der Putten 2014, Classen et al. 2015).

Issues with generalizations in exotic and range-expanding plant species: the role of novelty and relatedness in comparisons

Literature on the impact of introduced exotic species has widely focused on exotic species that become locally invasive in other continents while introduced plant species that are naturalized but not (yet) invasive are far less well studied. This has created a bias in the literature towards those exotic species that have the biggest impact on the recipient ecosystems (Pyšek et al. 2008). However, it has been shown that the percentage of introduced exotic species that become invasive is around one per cent according to the tens rule proposed by Williamson and Fitter (1996). Furthermore, among those species that do become invasive there are no universal impacts of exotic invasive plants on the recipient ecosystems (Vilà et al. 2011, Pyšek et al. 2012, Castro-Díez et al. 2014). While it seems clear that introduced exotic plant species can alter nutrient pools and soil processes as compared to the native state, the direction and strength of these effects depend on the context of the studied sites or the species comparisons used. A main question is, therefore, what makes some exotic species and not others become invasive and with the potential to alter soil processes to variable extents?

In the case of range expansion within continents, and based on the results of my thesis, range-expanders do not seem to differ consistently from related natives that co-occur in the same ecosystems. Based on phylogenetic controlled comparisons, I show that soil bacterial communities (Chapter 3), soil community functioning (Chapter 3 and 4) and plant-soil feedback (Chapter 4 ) are not affected differently by range-expanders than by congeneric related plant species that are native in the invaded ecosystems. Especially in Chapter 3, I conducted a greenhouse experiment to disentangle the effects of trait differences between plant species and site characteristics by growing range-expanders and related natives in the same soils that were novel to both. I then determined bacterial community composition and soil community functioning in the rhizosphere over time. My results showed that communities did not differ between the range expander and the 
congeneric native throughout plant development, suggesting that there are no general differences between range-expanding and native plant species.

Overall, generalizations about the impacts of both intercontinental exotic and intracontinental range-expanding plant species seem difficult to make, because very often impacts are highly context dependent. Key aspects behind this context dependency may be plant relatedness and trait novelty. Plant species that are less phylogenetically related to natives have been suggested to have higher invasive potential (Strauss et al. 2006), which may also be the case for range-expansions (Koorem et al. 2018). Unrelated exotic plant species are also more likely to bring specific plant traits into the ecosystem that are not present in the native community. Studies have shown that impacts of exotic species on soil communities and their functions are often strongest when compared species are more distant in their functional traits (Vilà et al. 2011) or bring in novel chemicals (Callaway and Ridenour 2004). For example, impacts of an exotic legume on nitrogen cycling in the soil will differ from impacts of non-nitrogen fixer (Castro-Díez et al. 2014), independent of its exotic origin. Besides traits, evolutionary novelty may shape the interactions of exotic species and soil organisms in the new range as well (Verhoeven et al. 2009, Lankau 2011). Therefore, the degree of evolutionary novelty may determine whether the exotic plant can be recognized by native soil biota and vice-versa, which may also contribute to the magnitude and type of impact of the particular species. Altogether, these factors that are characteristic to each specific study may play an important role in explaining the variation in impacts of exotic species. In my studies, I used phylogenetically controlled comparisons of congeneric plant species, which may have decreased variation of plant traits between compared species. Also, the evolutionary novelty of range expanders that have congeneric native species along the expansion range may be less than that of intercontinental exotics. Among range-expanders however, there can still be species-specific cases where rangeexpanders and related natives differ substantially in some specific effects, for example the range-expander Centaurea stoebe that has been shown to be less affected by root-feeding nematodes possibly due to its novel chemistry (Wilschut et al. 2017).

\section{The role of plant-soil interactions in plant range expansion}

The ecological consequences of plant range expansions are only starting to be investigated. Studying plant-soil interactions and feedbacks can help to understand the success and 
effects of plant range-expansion induced by climate warming (van der Putten 2012). In my thesis, I examined the role of indirect plant-soil interactions driven by plant inputs to the soil and the activity of the decomposer community. Often, I show that range-expanding plant species resemble related natives that occur in the same ecosystems, both in terms of their effect on soil communities (Chapter 3) and soil functioning (Chapter 3, 4 and 5). Through the different chapters, I showed that, in general, these indirect plant-soil interactions are not dependent on plant origin and thus are not a simple explanation of the success and consequences of range-expanding plants. Only for some species-specific cases, such as for the range expander Centaurea stoebe in Chapter 4, I suggest that positive litter feedbacks can potentially play a role on invasiveness in the longer term.

Nevertheless, direct plant-soil interactions, mediated by natural enemies and mutualists in the rhizosphere, may be important determining the success of range-expanding plant species. Previous studies have shown that plants perform better in soils of their new range than in soils from their original range (Van Grunsven et al. 2010, Dostálek et al. 2016) and are less affected by natural enemies in the new range (Engelkes et al. 2008, Morriën et al. 2012, Morriën et al. 2013). It is plausible that direct plant-soil interactions play a bigger role than indirect interactions, as their effects on plant performance are rapid and severe compared to those of decomposers and soil nutrients. Yet, the role of plant litter and nutrient availability in the soil may become important in combination with direct effects (Blumenthal et al. 2009) and in the long term, via the buildup of plant-soil feedback effects via plant litter (Eppinga and Molofsky 2013, Mariotte et al. 2017).

\section{Future research}

\section{Long-term plant-soil feedbacks through plant litter}

In my thesis, I suggest that some range-expanding plant species could potentially develop a positive feedback effect via plant litter inputs and litter decomposition in the soil (Chapter 4). The role of plant litter feedbacks, however, may become stronger and more relevant over time especially under field conditions where nutrient provisioning for plants depend on the amount and quality of litter and its decomposition (Wardle et al. 2004, van der Heijden et al. 2008). As suggested by the results presented in this thesis some rangeexpanding plant species may stimulate higher decomposition rates and some may also benefit from higher nutrient availability in the soil. Experiments addressing litter 
feedbacks in field conditions should preferably last for multiple growing seasons as litter feedbacks would only start to play a role after the first plant generation. Overall, longerterm experiments may enable litter feedbacks to be established and more accurately measured. An overarching hypothesis would be that if litter feedbacks play a role in the performance of range-expanding plants, effects are most likely to be quantified over longer conditioning times, when root litter is also included, or in interaction with extreme weather events such as summer drought.

Competition between range-expanding and native plant species

In order to understand plant species responses to climate change, it is essential to incorporate competitive interactions between novel exotic species and natives (Alexander et al. 2015, Alexander et al. 2016). When an exotic plant enter a novel community in the new range, it will encounter novel competitors. The outcome of such competitive interactions will determine the performance of the exotic species and its effects on native communities. Furthermore, competitive plant-plant interactions can strongly affect plantsoil feedback effects (Casper and Castelli 2007). In plant range expansions, the competitive interactions between range-expanding plant species and natives have received little attention (but see Koorem et al. (2018)). Therefore, in order to determine the establishment success and the effects of range-expanding plant species under field conditions in the new range, it will be important to assess their performance together with naturally occurring competitors and through the feedback effects resulting from such competition, including both related and unrelated plant species.

\section{Conclusions}

In my thesis, I show that plant species that expand their range as a result of climate warming in general are not species with higher nutrient content in their leaves and in the soils where they grow in the field as compared to native plant species. I conclude that there are no general patterns in plant and soil chemistry and nutrient content due to plant origin. Therefore, and similarly to exotic plant species, it remains a challenge to make accurate predictions of what range-expanding plant species, when or where may successfully establish in the new range.

Furthermore, I have shown that range-expanding plant species recruited similar bacterial rhizosphere communities as native species when both species are grown in the same soil. 
However, in some cases communities in soils of range-expanding plant species in the field may be different from communities in the soils of native species. All soil communities, however, shared similar ability to utilize organic substrates regardless of whether rangeexpanding or native plant species conditioned the soil and regardless of how similar bacterial communities were in those soils. Therefore, plant origin does neither explain variation in soil bacterial communities nor substrate utilization profiles, as soil communities were functionally redundant.

In the plant-soil feedback study, I demonstrated that plant-soil feedbacks are neutral between range-expanding and congeneric native plant species, both via rhizosphere and litter pathways. However, some range-expanding plants, such as Centaurea stoebe, could benefit from positive feedbacks via plant litter because its litter decomposes more than the litter of natives and it can grow more than the native Centaurea jacea under high nutrient availability. However, I have also determined that these features are not characteristic of range-expanding plants in general, but are particular to some plant genera and not to others.

My study has also shown that soils of range-expanding plant species may be less responsive to drought and rewetting cycles than soils of native plant communities, suggesting that the effects of plant-soil interactions on soil functioning may be more important under scenarios of climate extreme events. Although differences in the response of soil functions to drought and rewetting were most prominent in the short-term after drought, I emphasize that these dynamics may become more important in scenarios of recurrent drought events.

Overall, the results of my thesis suggest a limited role of plant origin and specialist decomposer communities on indirect plant-soil interactions of range-expanding plant species. When applying phylogenetic controlled comparisons, range-expanding and related native species do not differ in rhizosphere bacterial community composition, soil community functioning, and plant-soil feedback effects. Therefore, it seems unlikely that range-expanding species with congeneric natives will resemble exotic invasive species and will change general soil functioning in the new range, although local effects in the field may need to be quantified. 


\section{List of References}

Agrawal, A. A., P. M. Kotanen, C. E. Mitchell, A. G. Power, W. Godsoe, and J. Klironomos. 2005. Enemy release? An experiment with congeneric plant pairs and diverse above- and belowground enemies. Ecology 86:2979-2989.

Ahmed, M. A., M. Sanaullah, E. Blagodatskaya, K. Mason-Jones, H. Jawad, Y. Kuzyakov, and M. A. Dippold. 2018. Soil microorganisms exhibit enzymatic and priming response to root mucilage under drought. Soil Biology and Biochemistry 116:410-418.

Alba, C., J. E. NeSmith, C. Fahey, C. Angelini, and S. L. Flory. 2017. Methods to test the interactive effects of drought and plant invasion on ecosystem structure and function using complementary common garden and field experiments. Ecology and Evolution 7:1442-1452.

Alexander, J. M., J. M. Diez, S. P. Hart, and J. M. Levine. 2016. When Climate Reshuffles Competitors: A Call for Experimental Macroecology. Trends in Ecology \& Evolution 31:831-841.

Alexander, J. M., J. M. Diez, and J. M. Levine. 2015. Novel competitors shape species' responses to climate change. Nature 525:515-518.

Allison, S. D., and J. B. H. Martiny. 2008. Resistance, resilience, and redundancy in microbial communities. Proceedings of the National Academy of Sciences of the United States of America 105:11512-11519.

Allison, S. D., C. Nielsen, and R. F. Hughes. 2006. Elevated enzyme activities in soils under the invasive nitrogen-fixing tree Falcataria moluccana. Soil Biology and Biochemistry 38:1537-1544.

Allison, S. D., and P. M. Vitousek. 2004. Rapid nutrient cycling in leaf litter from invasive plants in Hawai'i. Oecologia 141:612-619.

Almagro, M., J. López, J. I. Querejeta, and M. Martínez-Mena. 2009. Temperature dependence of soil $\mathrm{CO} 2$ efflux is strongly modulated by seasonal patterns of moisture availability in a Mediterranean ecosystem. Soil Biology and Biochemistry 41:594-605.

Anacker, B. L., J. N. Klironomos, H. Maherali, K. O. Reinhart, and S. Y. Strauss. 2014. Phylogenetic conservatism in plant-soil feedback and its implications for plant abundance. Ecology Letters 17:1613-1621.

Anderson, M. J. 2006. Distance-Based Tests for Homogeneity of Multivariate Dispersions. Biometrics 62:245-253.

Andonian, K., J. L. Hierro, L. Khetsuriani, P. Becerra, G. Janoyan, D. Villarreal, L. Cavieres, L. R. Fox, and R. M. Callaway. 2011. Range-Expanding Populations of a Globally Introduced Weed Experience Negative Plant-Soil Feedbacks. Plos One 6.

Arthur, M. A., S. R. Bray, C. R. Kuchle, and R. W. McEwan. 2012. The influence of the invasive shrub, Lonicera maackii, on leaf decomposition and microbial community dynamics. Plant Ecology 213:1571-1582.

Ashton, I. W., L. A. Hyatt, K. M. Howe, J. Gurevitch, and M. T. Lerdau. 2005. Invasive species accelerate decomposition and litter nitrogen loss in a mixed deciduous forest. Ecological Applications 15:1263-1272.

Austin, A. T., L. Vivanco, A. González-Arzac, and L. I. Pérez. 2014. There's no place like home? An exploration of the mechanisms behind plant litter-decomposer affinity in terrestrial ecosystems. New Phytologist 204:307-314.

Averill, C., B. G. Waring, and C. V. Hawkes. 2016. Historical precipitation predictably alters the shape and magnitude of microbial functional response to soil moisture. Global Change Biology 22:1957-1964.

Ayres, E., H. Steltzer, S. Berg, and D. H. Wall. 2009a. Soil biota accelerate decomposition in highelevation forests by specializing in the breakdown of litter produced by the plant species above them. Journal of Ecology 97:901-912.

Ayres, E., H. Steltzer, B. L. Simmons, R. T. Simpson, J. M. Steinweg, M. D. Wallenstein, N. Mellor, W. J. Parton, J. C. Moore, and D. H. Wall. 2009b. Home-field advantage accelerates leaf litter decomposition in forests. Soil Biology and Biochemistry 41:606-610. 
Badri, D. V., and J. M. Vivanco. 2009. Regulation and function of root exudates. Plant, Cell \& Environment 32:666-681.

Bailly, A., and L. Weisskopf. 2012. The modulating effect of bacterial volatiles on plant growth. Plant Signaling \& Behavior 7:79-85.

Baldrian, P. 2009. Microbial enzyme-catalyzed processes in soils and their analysis. Plant, Soil and Environment 55:370-378.

Bardgett, R. D., C. Freeman, and N. J. Ostle. 2008. Microbial contributions to climate change through carbon cycle feedbacks. ISME J 2:805-814.

Bardgett, R. D., P. Manning, E. Morriën, and F. T. Vries. 2013. Hierarchical responses of plant-soil interactions to climate change: consequences for the global carbon cycle. Journal of Ecology 101:334-343.

Bardgett, R. D., and W. H. van der Putten. 2014. Belowground biodiversity and ecosystem functioning. Nature 515:505-511.

Barnard, R. L., C. A. Osborne, and M. K. Firestone. 2013. Responses of soil bacterial and fungal communities to extreme desiccation and rewetting. The ISME Journal 7:2229-2241.

Berg, G., and K. Smalla. 2009. Plant species and soil type cooperatively shape the structure and function of microbial communities in the rhizosphere. FEMS Microbiol Ecol 68:1-13.

Berg, M. P., E. T. Kiers, G. Driessen, M. van der Heijden, B. W. Kooi, F. Kuenen, M. Liefting, H. A. Verhoef, and J. Ellers. 2010. Adapt or disperse: understanding species persistence in a changing world. Global Change Biology 16:587-598.

Bever, J. D., K. M. Westover, and J. Antonovics. 1997. Incorporating the Soil Community into Plant Population Dynamics: The Utility of the Feedback Approach. Journal of Ecology 85:561-573.

Bezemer, T. M., S. Lawson Clare, K. Hedlund, R. Edwards Andrew, J. Brook Alex, M. Igual JosÉ, R. Mortimer Simon, and H. van der Putten Wim. 2006. Plant species and functional group effects on abiotic and microbial soil properties and plant-soil feedback responses in two grasslands. Journal of Ecology 94:893-904.

Birch, H. F. 1958. The effect of soil drying on humus decomposition and nitrogen availability. Plant and Soil 10:9-31.

Birgander, J., J. Rousk, and P. A. Olsson. 2017. Warmer winters increase the rhizosphere carbon flow to mycorrhizal fungi more than to other microorganisms in a temperate grassland. Global Change Biology 23:5372-5382.

Birouste, M., E. Kazakou, A. Blanchard, and C. Roumet. 2012. Plant traits and decomposition: are the relationships for roots comparable to those for leaves? Ann Bot 109:463-472.

Blumenthal, D., C. E. Mitchell, P. Pyšek, and V. Jarošík. 2009. Synergy between pathogen release and resource availability in plant invasion. Proceedings of the National Academy of Sciences 106:7899-7904.

Bradford, M. A., M. S. Strickland, J. L. DeVore, and J. C. Maerz. 2012. Root carbon flow from an invasive plant to belowground foodwebs. Plant and Soil 359:233-244.

Bradford, M. A., G. F. Veen, A. Bonis, E. M. Bradford, A. T. Classen, J. H. C. Cornelissen, T. W. Crowther, J. R. De Long, G. T. Freschet, P. Kardol, M. Manrubia-Freixa, D. S. Maynard, G. S. Newman, R. S. P. Logtestijn, M. Viketoft, D. A. Wardle, W. R. Wieder, S. A. Wood, and W. H. van der Putten. 2017. A test of the hierarchical model of litter decomposition. Nature Ecology \& Evolution 1:1836-1845.

Brinkman, E. P., W. H. van der Putten, E.-J. Bakker, and K. J. F. Verhoeven. 2010. Plant-soil feedback: experimental approaches, statistical analyses and ecological interpretations. Journal of Ecology 98:1063-1073.

Bushnell, B. 2015. BBMap. http://sourceforge.net/projects/bbmap/.

Caldeira, M. C., X. Lecomte, T. S. David, J. G. Pinto, M. N. Bugalho, and C. Werner. 2015. Synergy of extreme drought and shrub invasion reduce ecosystem functioning and resilience in waterlimited climates. Scientific Reports 5:15110.

Callaway, R. M., and W. M. Ridenour. 2004. Novel weapons: invasive success and the evolution of increased competitive ability. Frontiers in Ecology and the Environment 2:436-443. 
Callaway, R. M., G. C. Thelen, A. Rodriguez, and W. E. Holben. 2004. Soil biota and exotic plant invasion. Nature 427:731-733.

Caporaso, J. G., C. L. Lauber, W. A. Walters, D. Berg-Lyons, J. Huntley, N. Fierer, S. M. Owens, J. Betley, L. Fraser, M. Bauer, N. Gormley, J. A. Gilbert, G. Smith, and R. Knight. 2012. Ultrahigh-throughput microbial community analysis on the Illumina HiSeq and MiSeq platforms. ISME J 6:1621-1624.

Cappuccino, N., and J. Arnason. 2006. Novel chemistry of invasive exotic plants. Biology Letters 2:189-193.

Carney, K. M., and P. A. Matson. 2005. Plant Communities, Soil Microorganisms, and Soil Carbon Cycling: Does Altering the World Belowground Matter to Ecosystem Functioning? Ecosystems 8:928-940.

Casper, B. B., and J. P. Castelli. 2007. Evaluating plant-soil feedback together with competition in a serpentine grassland. Ecology Letters 10:394-400.

Castro-Díez, P., and Á. Alonso. 2017. Alteration of Nitrogen Cycling as a Result of Invasion. Pages 4962 in M. Vilà and P. E. Hulme, editors. Impact of Biological Invasions on Ecosystem Services. Springer International Publishing, Cham.

Castro-Díez, P., O. Godoy, A. Alonso, A. Gallardo, and A. Saldaña. 2014. What explains variation in the impacts of exotic plant invasions on the nitrogen cycle? A meta-analysis. Ecology Letters 17:1-12.

Chen, I. C., J. K. Hill, R. Ohlemuller, D. B. Roy, and C. D. Thomas. 2011. Rapid range shifts of species associated with high levels of climate warming. Science 333:1024-1026.

Cheng, W., D. W. Johnson, and S. Fu. 2003. Rhizosphere Effects on Decomposition. Soil Sci. Soc. Am. J. 67:1418-1427.

Chomel, M., C. Fernandez, A. Bousquet-Mélou, C. Gers, Y. Monnier, M. Santonja, T. Gauquelin, R. Gros, C. Lecareux, V. Baldy, and A. Austin. 2014. Secondary metabolites of Pinus halepensis alter decomposer organisms and litter decomposition during afforestation of abandoned agricultural zones. Journal of Ecology 102:411-424.

Ciais, P., M. Reichstein, N. Viovy, A. Granier, J. Ogée, V. Allard, M. Aubinet, N. Buchmann, C. Bernhofer, A. Carrara, F. Chevallier, N. De Noblet, A. D. Friend, P. Friedlingstein, T. Grünwald, B. Heinesch, P. Keronen, A. Knohl, G. Krinner, D. Loustau, G. Manca, G. Matteucci, F. Miglietta, J. M. Ourcival, D. Papale, K. Pilegaard, S. Rambal, G. Seufert, J. F. Soussana, M. J. Sanz, E. D. Schulze, T. Vesala, and R. Valentini. 2005. Europe-wide reduction in primary productivity caused by the heat and drought in 2003. Nature 437:529.

Classen, A. T., M. K. Sundqvist, J. A. Henning, G. S. Newman, J. A. M. Moore, M. A. Cregger, L. C. Moorhead, and C. M. Patterson. 2015. Direct and indirect effects of climate change on soil microbial and soil microbial-plant interactions: What lies ahead? Ecosphere 6:1-21.

Cleland, E. E., I. Chuine, A. Menzel, H. A. Mooney, and M. D. Schwartz. 2007. Shifting plant phenology in response to global change. Trends in Ecology \& Evolution 22:357-365.

Cole, J. R., Q. Wang, J. A. Fish, B. Chai, D. M. McGarrell, Y. Sun, C. T. Brown, A. Porras-Alfaro, C. R. Kuske, and J. M. Tiedje. 2014. Ribosomal Database Project: data and tools for high throughput rRNA analysis. Nucleic Acids Research 42:D633-D642.

Collins, C. G., C. J. Carey, E. L. Aronson, C. W. Kopp, and J. M. Diez. 2016. Direct and indirect effects of native range expansion on soil microbial community structure and function. Journal of Ecology 104:1271-1283.

Cornelissen, J. H. C., H. M. Quested, D. Gwynn-Jones, R. S. P. Van Logtestijn, M. A. H. De Beus, A. Kondratchuk, T. V. Callaghan, and R. Aerts. 2004. Leaf digestibility and litter decomposability are related in a wide range of subarctic plant species and types. Functional Ecology 18:779786.

Cornwell, W. K., J. H. Cornelissen, K. Amatangelo, E. Dorrepaal, V. T. Eviner, O. Godoy, S. E. Hobbie, B. Hoorens, H. Kurokawa, N. Perez-Harguindeguy, H. M. Quested, L. S. Santiago, D. A. Wardle, I. J. Wright, R. Aerts, S. D. Allison, P. van Bodegom, V. Brovkin, A. Chatain, T. V. Callaghan, S. Diaz, E. Garnier, D. E. Gurvich, E. Kazakou, J. A. Klein, J. Read, P. B. Reich, N. A. Soudzilovskaia, 
M. V. Vaieretti, and M. Westoby. 2008. Plant species traits are the predominant control on litter decomposition rates within biomes worldwide. Ecol Lett 11:1065-1071.

Crowther, T. W., S. M. Thomas, D. S. Maynard, P. Baldrian, K. Covey, S. D. Frey, L. T. A. van Diepen, and M. A. Bradford. 2015. Biotic interactions mediate soil microbial feedbacks to climate change. Proceedings of the National Academy of Sciences.

Crowther, T. W., K. E. O. Todd-Brown, C. W. Rowe, W. R. Wieder, J. C. Carey, M. B. Machmuller, B. L. Snoek, S. Fang, G. Zhou, S. D. Allison, J. M. Blair, S. D. Bridgham, A. J. Burton, Y. Carrillo, P. B. Reich, J. S. Clark, A. T. Classen, F. A. Dijkstra, B. Elberling, B. A. Emmett, M. Estiarte, S. D. Frey, J. Guo, J. Harte, L. Jiang, B. R. Johnson, G. Kröel-Dulay, K. S. Larsen, H. Laudon, J. M. Lavallee, Y. Luo, M. Lupascu, L. N. Ma, S. Marhan, A. Michelsen, J. Mohan, S. Niu, E. Pendall, J. Peñuelas, L. Pfeifer-Meister, C. Poll, S. Reinsch, L. L. Reynolds, I. K. Schmidt, S. Sistla, N. W. Sokol, P. H. Templer, K. K. Treseder, J. M. Welker, and M. A. Bradford. 2016. Quantifying global soil carbon losses in response to warming. Nature 540:104.

Dassen, S., R. Cortois, H. Martens, M. Hollander, G. A. Kowalchuk, W. H. van der Putten, and G. B. D. Deyn. 2017. Differential responses of soil bacteria, fungi, archaea and protists to plant species richness and plant functional group identity. Molecular Ecology 26:4085-4098.

Davis, M. B., and R. G. Shaw. 2001. Range shifts and adaptive responses to Quaternary climate change. Science 292:673-679.

De Deyn, G. B., J. H. Cornelissen, and R. D. Bardgett. 2008. Plant functional traits and soil carbon sequestration in contrasting biomes. Ecol Lett 11:516-531.

De Deyn, G. B., and W. H. Van der Putten. 2005. Linking aboveground and belowground diversity. Trends Ecol Evol 20:625-633.

de Graaf, M., K. van Groenigen, J. Six, B. Hungate, and C. van Kessel. 2006. Interactions between plant growth and soil nutrient cycling under elevated CO2: a meta-analysis. Global Change Biology 12:2077-2091.

de Graaff, M. A., A. T. Classen, H. F. Castro, and C. W. Schadt. 2010. Labile soil carbon inputs mediate the soil microbial community composition and plant residue decomposition rates. New Phytol 188:1055-1064.

de Hollander, M. 2017. nioo-knaw/hydra: 1.3.3 (Version 1.3.3). Zenodo. http://doi.org/10.5281/zenodo.884028.

de Ridder-Duine, A. S., G. A. Kowalchuk, P. J. A. Klein Gunnewiek, W. Smant, J. A. van Veen, and W. de Boer. 2005. Rhizosphere bacterial community composition in natural stands of Carex arenaria (sand sedge) is determined by bulk soil community composition. Soil Biology and Biochemistry 37:349-357.

de Vries, F. T., M. E. Liiri, L. Bjørnlund, H. M. Setälä, S. Christensen, and R. D. Bardgett. 2012a. Legacy effects of drought on plant growth and the soil food web. Oecologia 170:821-833.

de Vries, F. T., P. Manning, J. R. Tallowin, S. R. Mortimer, E. S. Pilgrim, K. A. Harrison, P. J. Hobbs, H. Quirk, B. Shipley, J. H. Cornelissen, J. Kattge, and R. D. Bardgett. 2012b. Abiotic drivers and plant traits explain landscape-scale patterns in soil microbial communities. Ecol Lett 15:12301239.

de Vries, F. T., and A. Shade. 2013. Controls on soil microbial community stability under climate change. Front Microbiol 4:265.

Delgado-Baquerizo, M., A. M. Oliverio, T. E. Brewer, A. Benavent-González, D. J. Eldridge, R. D. Bardgett, F. T. Maestre, B. K. Singh, and N. Fierer. 2018. A global atlas of the dominant bacteria found in soil. Science 359:320-325.

Diez, J. M., I. Dickie, G. Edwards, P. E. Hulme, J. J. Sullivan, and R. P. Duncan. 2010. Negative soil feedbacks accumulate over time for non-native plant species. Ecol Lett 13:803-809.

Dodt, M., J. Roehr, R. Ahmed, and C. Dieterich. 2012. FLEXBAR-Flexible Barcode and Adapter Processing for Next-Generation Sequencing Platforms. Biology 1:895.

Dostál, P., J. Müllerová, P. Pyšek, J. Pergl, and T. Klinerová. 2013. The impact of an invasive plant changes over time. Ecology Letters 16:1277-1284. 
Dostálek, T., Z. Münzbergová, A. Kladivová, and M. Macel. 2016. Plant-soil feedback in native vs. invasive populations of a range expanding plant. Plant and Soil 399:209-220.

Drigo, B., G. A. Kowalchuk, E. Yergeau, T. M. Bezemer, H. T. S. Boschker, and J. A. Van Veen. 2007. Impact of elevated carbon dioxide on the rhizosphere communities of Carex arenaria and Festuca rubra. Global Change Biology 13:2396-2410.

Dukes, J. S., and B. A. Hungate. 2002. Elevated Carbon Dioxide and Litter Decomposition in California Annual Grasslands: Which Mechanisms Matter? Ecosystems 5:171-183.

Edgar, R. C. 2010. Search and clustering orders of magnitude faster than BLAST. Bioinformatics 26:2460-2461.

Edgar, R. C., Haas, B.J., Clemente, J.C., Quince, C., Knight, R. 2011. UCHIME [WWW Document]. URL http://www.drive5.com/uchime/ (accessed 6.20.11).

EEA. 2016. Meteorological and hydrological droughts. European Environment Agency, Copenhagen.

Ehrenfeld, J. G. 2003. Effects of Exotic Plant Invasions on Soil Nutrient Cycling Processes. Ecosystems 6:503-523.

Ehrenfeld, J. G. 2010. Ecosystem Consequences of Biological Invasions. Annual Review of Ecology, Evolution, and Systematics 41:59-80.

Ehrenfeld, J. G., P. Kourtev, and W. Huang. 2001. Changes in soil functions following invasions of exotic understory plants in decidious forests. Ecological Applications 11:1287-1300.

Ehrenfeld, J. G., B. Ravit, and K. Elgersma. 2005. Feedback in the Plant-Soil System. Annual Review of Environment and Resources 30:75-115.

Eilers, K. G., C. L. Lauber, R. Knight, and N. Fierer. 2010. Shifts in bacterial community structure associated with inputs of low molecular weight carbon compounds to soil. Soil Biology and Biochemistry 42:896-903.

Elgersma, K. J., S. Yu, T. Vor, and J. G. Ehrenfeld. 2012. Microbial-mediated feedbacks of leaf litter on invasive plant growth and interspecific competition. Plant and Soil 356:341-355.

Engelkes, T., E. Morriën, K. J. Verhoeven, T. M. Bezemer, A. Biere, J. A. Harvey, L. M. McIntyre, W. L. Tamis, and W. H. van der Putten. 2008. Successful range-expanding plants experience less above-ground and below-ground enemy impact. Nature 456:946-948.

Eppinga, M. B., M. A. Kaproth, A. R. Collins, and J. Molofsky. 2011. Litter feedbacks, evolutionary change and exotic plant invasion. Journal of Ecology 99:503-514.

Eppinga, M. B., and J. Molofsky. 2013. Eco-evolutionary litter feedback as a driver of exotic plant invasion. Perspectives in Plant Ecology, Evolution and Systematics 15:20-31.

Evans, J. A., R. A. Lankau, A. S. Davis, S. Raghu, and D. A. Landis. 2016. Soil-mediated eco-evolutionary feedbacks in the invasive plant Alliaria petiolata. Functional Ecology 30:1053-1061.

Fierer, N., and R. B. Jackson. 2006. The diversity and biogeography of soil bacterial communities. Proceedings of the National Academy of Sciences of the United States of America 103:626631.

Fierer, N., C. L. Lauber, K. S. Ramirez, J. Zaneveld, M. A. Bradford, and R. Knight. 2012. Comparative metagenomic, phylogenetic and physiological analyses of soil microbial communities across nitrogen gradients. ISME J 6:1007-1017.

Fierer, N., and J. P. Schimel. 2002. Effects of drying-rewetting frequency on soil carbon and nitrogen transformations. Soil Biology and Biochemistry 34:777-787.

Fierer, N., J. P. Schimel, and P. A. Holden. 2003. Influence of Drying-Rewetting Frequency on Soil Bacterial Community Structure. Microbial Ecology 45:63-71.

Franklin, R. B., and A. L. Mills. 2006. Structural and Functional Responses of a Sewage Microbial Community to Dilution-Induced Reductions in Diversity. Microbial Ecology 52:280-288.

Freschet, G. T., R. Aerts, and J. H. C. Cornelissen. 2012. Multiple mechanisms for trait effects on litter decomposition: moving beyond home-field advantage with a new hypothesis. Journal of Ecology 100:619-630.

Freschet, G. T., W. K. Cornwell, D. A. Wardle, T. G. Elumeeva, W. Liu, B. G. Jackson, V. G. Onipchenko, N. A. Soudzilovskaia, J. Tao, J. H. C. Cornelissen, and A. Austin. 2013. Linking litter 
decomposition of above- and below-ground organs to plant-soil feedbacks worldwide. Journal of Ecology 101:943-952.

Fuchslueger, L., M. Bahn, K. Fritz, R. Hasibeder, and A. Richter. 2014. Experimental drought reduces the transfer of recently fixed plant carbon to soil microbes and alters the bacterial community composition in a mountain meadow. New Phytologist 201:916-927.

Funk, J. L. 2008. Differences in plasticity between invasive and native plants from a low resource environment. Journal of Ecology 96:1162-1173.

Funk, J. L., and H. L. Throop. 2010. Enemy release and plant invasion: patterns of defensive traits and leaf damage in Hawaii. Oecologia 162:815-823.

Funk, J. L., and P. M. Vitousek. 2007. Resource-use efficiency and plant invasion in low-resource systems. Nature 446:1079-1081.

Garten, C. T., A. T. Classen, and R. J. Norby. 2009. Soil moisture surpasses elevated CO2 and temperature as a control on soil carbon dynamics in a multi-factor climate change experiment. Plant and Soil 319:85-94.

Gholz, H. L., D. A. Wedin, S. M. Smitherman, M. E. Harmon, and W. J. Parton. 2000. Long-term dynamics of pine and hardwood litter in contrasting environments: toward a global model of decomposition. Global Change Biology 6.

Gibbons, S. M., Y. Lekberg, D. L. Mummey, N. Sangwan, P. W. Ramsey, and J. A. Gilbert. 2017. Invasive Plants Rapidly Reshape Soil Properties in a Grassland Ecosystem. mSystems 2.

Gómez-Aparicio, L., and C. D. Canham. 2008. Neighborhood models of the effects of invasive tree species on ecosystem processes. Ecological Monographs 78:69-86.

Göransson, H., D. L. Godbold, D. L. Jones, and J. Rousk. 2013. Bacterial growth and respiration responses upon rewetting dry forest soils: Impact of drought-legacy. Soil Biology and Biochemistry 57:477-486.

Griffiths, B. S., and L. Philippot. 2013. Insights into the resistance and resilience of the soil microbial community. FEMS Microbiol Rev 37:112-129.

Griffiths, B. S., K. Ritz, R. D. Bardgett, R. Cook, S. Christensen, F. Ekelund, S. J. Sørensen, E. Bååth, J. Bloem, P. C. De Ruiter, J. Dolfing, and B. Nicolardot. 2000. Ecosystem response of pasture soil communities to fumigation-induced microbial diversity reductions: an examination of the biodiversity-ecosystem function relationship. Oikos 90:279-294.

Güsewell, S., and M. O. Gessner. 2009. N : P ratios influence litter decomposition and colonization by fungi and bacteria in microcosms. Functional Ecology 23:211-219.

Hannula, S. E., E. Morriën, M. de Hollander, W. H. van der Putten, J. A. van Veen, and W. de Boer. 2017. Shifts in rhizosphere fungal community during secondary succession following abandonment from agriculture. ISME J 11:2294-2304.

Harkes, P., A. Verhoeven, M. G. Sterken, L. B. Snoek, S. J. J. van den Elsen, P. J. W. Mooijman, C. W. Quist, M. T. W. Vervoort, and J. Helder. 2017. The differential impact of a native and a nonnative ragwort species (Senecioneae) on the first and second trophic level of the rhizosphere food web. Oikos 126:1790-1803.

Hawkes, C. V., and T. H. Keitt. 2015. Resilience vs. historical contingency in microbial responses to environmental change. Ecol Lett 18:612-625.

Hawkes, C. V., B. G. Waring, J. D. Rocca, and S. N. Kivlin. 2017. Historical climate controls soil respiration responses to current soil moisture. Proceedings of the National Academy of Sciences 114:6322-6327.

Hawkes, C. V., I. F. Wren, D. J. Herman, and M. K. Firestone. 2005. Plant invasion alters nitrogen cycling by modifying the soil nitrifying community. Ecology Letters 8:976-985.

Hayes, K. R., and S. C. Barry. 2008. Are there any consistent predictors of invasion success? Biological Invasions 10:483-506.

Heinen, R., M. Sluijs, A. Biere, J. A. Harvey, and T. M. Bezemer. 2017. Plant community composition but not plant traits determine the outcome of soil legacy effects on plants and insects. Journal of Ecology 00:1-13. 
Ihrmark, K., I. T. M. Bödeker, K. Cruz-Martinez, H. Friberg, A. Kubartova, J. Schenck, Y. Strid, J. Stenlid, M. Brandström-Durling, K. E. Clemmensen, and B. D. Lindahl. 2012. New primers to amplify the fungal ITS2 region - evaluation by 454-sequencing of artificial and natural communities. FEMS Microbiology Ecology 82:666-677.

Inderjit, E. Heather, C. Christoph, B. Devika, K. Rajwant, F. Yu-Long, S. Carlos, C. J. Treviño, V.-B. Alfonso, G. Jonathan, and C. R. M. 2011. Volatile chemicals from leaf litter are associated with invasiveness of a Neotropical weed in Asia. Ecology 92:316-324.

IPCC. 2014. Climate Change 2014: Synthesis Report. Contribution of Working Groups I, II and III to the Fifth Assessment Report of the Intergovernmental Panel on Climate Change. IPCC, Geneva, Switzerland.

Jump, A. S., and J. Penuelas. 2005. Running to stand still: adaptation and the response of plants to rapid climate change. Ecology Letters 8:1010-1020.

Kardol, P., T. M. Bezemer, and W. H. v. d. Putten. 2006. Temporal variation in plant-soil feedback controls succession. Ecology Letters 9:1080-1088.

Kardol, P., M. A. Cregger, C. E. Campany, and A. T. Classen. 2010. Soil ecosystem functioning under climate change: plant species and community effects. Ecology 91:767-781.

Ke, P.-J., T. Miki, and T.-S. Ding. 2015. The soil microbial community predicts the importance of plant traits in plant-soil feedback. New Phytologist 206:329-341.

Keane, R. M., and M. J. Crawley. 2002. Exotic plant invasions and the enemy release hypothesis. Trends in Ecology \& Evolution 17:164-170.

Keiser, A. D., M. S. Strickland, N. Fierer, and M. A. Bradford. 2011. The effect of resource history on the functioning of soil microbial communities is maintained across time. Biogeosciences 8:1477-1486.

Keuskamp, J. A., B. J. J. Dingemans, T. Lehtinen, J. M. Sarneel, and M. M. Hefting. 2013. Tea Bag Index: a novel approach to collect uniform decomposition data across ecosystems. Methods in Ecology and Evolution 4:1070-1075.

Klironomos, J. N. 2002. Feedback with soil biota contributes to plant rarity and invasiveness in communities. Nature 417:67-70.

KNMI. 2015. KNMI'14-klimaatscenario's voor Nederland; Leidraad voor professionals in klimaatadaptatie. De Bilt.

Kõljalg, U., R. H. Nilsson, K. Abarenkov, L. Tedersoo, A. F. S. Taylor, M. Bahram, S. T. Bates, T. D. Bruns, J. Bengtsson-Palme, T. M. Callaghan, B. Douglas, T. Drenkhan, U. Eberhardt, M. Dueñas, T. Grebenc, G. W. Griffith, M. Hartmann, P. M. Kirk, P. Kohout, E. Larsson, B. D. Lindahl, R. Lücking, M. P. Martín, P. B. Matheny, N. H. Nguyen, T. Niskanen, J. Oja, K. G. Peay, U. Peintner, M. Peterson, K. Põldmaa, L. Saag, I. Saar, A. Schüßler, J. A. Scott, C. Senés, M. E. Smith, A. Suija, D. L. Taylor, M. T. Telleria, M. Weiss, and K.-H. Larsson. 2013. Towards a unified paradigm for sequence-based identification of fungi. Molecular Ecology 22:52715277.

Komsta, L. 2011. outliers: Test for outliers.

Koorem, K., O. Kostenko, L. B. Snoek, C. Weser, K. S. Ramirez, R. A. Wilschut, and W. H. v. d. Putten. 2018. Relatedness with plant species in native community influences ecological consequences of range expansions. Oikos 127:981-990.

Köster, J., and S. Rahmann. 2012. Snakemake-a scalable bioinformatics workflow engine. Bioinformatics 28:2520-2522.

Kourtev, P., J. Ehrenfeld, and M. Häggblom. 2002a. Exotic plant species alter the microbial community structure and function in the soil. Ecology 83:3152-3166.

Kourtev, P., J. Ehrenfeld, and M. Häggblom. 2003. Experimental analysis of the effect of exotic and native plant species on the structure and function of soil microbial communities. Soil Biology and Biochemistry 35:895-905.

Kourtev, P., J. Ehrenfeld, and W. Huang. 2002b. Enzyme activities during litter decomposition of two exotic and two native plant species in hardwood forests of New Jersey. Soil Biology and Biochemistry 34:1207-1218. 
Kowalchuk, G. A., D. S. Buma, W. de Boer, P. G. L. Klinkhamer, and J. A. van Veen. 2002. Effects of above-ground plant species composition and diversity on the diversity of soil-borne microorganisms. Antonie van Leeuwenhoek 81:509.

Kulmatiski, A., K. H. Beard, J. R. Stevens, and S. M. Cobbold. 2008. Plant-soil feedbacks: a metaanalytical review. Ecology Letters 11:980-992.

Kuznetsova, A., P. B. Brockhoff, and R. H. B. Christensen. 2013. ImerTest.

Kuzyakov, Y., P. W. Hill, and D. L. Jones. 2006. Root exudate components change litter decomposition in a simulated rhizosphere depending on temperature. Plant and Soil 290:293-305.

Lankau, R. A. 2011. Resistance and recovery of soil microbial communities in the face of Alliaria petiolata invasions. New Phytologist 189:536-548.

Lankau, R. A., V. Nuzzo, G. Spyreas, and A. S. Davis. 2009. Evolutionary limits ameliorate the negative impact of an invasive plant. Proceedings of the National Academy of Sciences 106:1536215367.

Lee, M. R., E. S. Bernhardt, P. M. v. Bodegom, J. H. C. Cornelissen, J. Kattge, D. C. Laughlin, Ü. Niinemets, J. Peñuelas, P. B. Reich, B. Yguel, and J. P. Wright. 2017. Invasive species' leaf traits and dissimilarity from natives shape their impact on nitrogen cycling: a meta-analysis. New Phytologist 213:128-139.

Legay, N., C. Baxendale, K. Grigulis, U. Krainer, E. Kastl, M. Schloter, R. D. Bardgett, C. Arnoldi, M. Bahn, M. Dumont, F. Poly, T. Pommier, J. C. Clément, and S. Lavorel. 2014. Contribution of above- and below-ground plant traits to the structure and function of grassland soil microbial communities. Annals of Botany 114:1011-1021.

Lekberg, Y., J. D. Bever, R. A. Bunn, R. M. Callaway, M. M. Hart, S. N. Kivlin, J. Klironomos, B. G. Larkin, J. L. Maron, K. O. Reinhart, M. Remke, and W. H. Putten. 2018. Relative importance of competition and plant-soil feedback, their synergy, context dependency and implications for coexistence. Ecology Letters 21:1268-1281.

Lenoir, J., J. C. Gegout, P. A. Marquet, P. de Ruffray, and H. Brisse. 2008. A significant upward shift in plant species optimum elevation during the 20th century. Science 320:1768-1771.

Lenth, R. V. 2016. Least-Squares Means: The R Package Ismeans. Journal of Statistical Software 69:133.

Levine, J. M., P. B. Adler, and S. G. Yelenik. 2004. A meta-analysis of biotic resistance to exotic plant invasions. Ecology Letters 7:975-989.

Levine, J. M., M. Vilà, C. M. D. Antonio, J. S. Dukes, K. Grigulis, and S. Lavorel. 2003. Mechanisms underlying the impacts of exotic plant invasions. Proceedings of the Royal Society of London. Series B: Biological Sciences 270:775-781.

Liao, C., R. Peng, Y. Luo, X. Zhou, X. Wu, C. Fang, J. Chen, and B. Li. 2008. Altered ecosystem carbon and nitrogen cycles by plant invasion: a meta-analysis. New Phytologist 177:706-714.

Liu, Y., J. Li, Y. Jin, Y. Zhang, L. Sha, J. Grace, Q. Song, W. Zhou, A. Chen, P. Li, and S. Zhang. 2018. The influence of drought strength on soil respiration in a woody savanna ecosystem, southwest China. Plant and Soil 428:321-333.

Liu, Y., and M. van Kleunen. 2017. Responses of common and rare aliens and natives to nutrient availability and fluctuations. Journal of Ecology 105:1111-1122.

Ma, C., Y. Xiong, L. Li, and D. Guo. 2016. Root and leaf decomposition become decoupled over time: implications for below- and above-ground relationships. Functional Ecology 30:1239-1246.

Ma, H.-K., A. Pineda, A. W. G. van der Wurff, C. Raaijmakers, and T. M. Bezemer. 2017. Plant-Soil Feedback Effects on Growth, Defense and Susceptibility to a Soil-Borne Disease in a Cut Flower Crop: Species and Functional Group Effects. Frontiers in Plant Science 8.

Macel, M., R. C. H. de Vos, J. J. Jansen, W. H. van der Putten, and N. M. van Dam. 2014. Novel chemistry of invasive plants: exotic species have more unique metabolomic profiles than native congeners. Ecology and Evolution 4:2777-2786.

Madritch, M. D., and R. L. Lindroth. 2011. Soil microbial communities adapt to genetic variation in leaf litter inputs. Oikos 120:1696-1704. 
Manzoni, S., J. P. Schimel, and A. Porporato. 2012. Responses of soil microbial communities to water stress: results from a meta-analysis. Ecology 93:930-938.

Mariotte, P., E. N. Spotswood, E. C. Farrer, K. N. Suding, and L. Fraser. 2017. Positive litter feedbacks of an introduced species reduce native diversity and promote invasion in Californian grasslands. Applied Vegetation Science 20:28-39.

Masella, A. P., A. K. Bartram, J. M. Truszkowski, D. G. Brown, and J. D. Neufeld. 2012. PANDAseq: paired-end assembler for illumina sequences. BMC Bioinformatics 13:31.

Matteucci, M., C. Gruening, I. Goded Ballarin, G. Seufert, and A. Cescatti. 2015. Components, drivers and temporal dynamics of ecosystem respiration in a Mediterranean pine forest. Soil Biology and Biochemistry 88:224-235.

Mazzoleni, S., G. Bonanomi, G. Incerti, M. L. Chiusano, P. Termolino, A. Mingo, M. Senatore, F. Giannino, F. Cartenì, M. Rietkerk, and V. Lanzotti. 2015. Inhibitory and toxic effects of extracellular self-DNA in litter: a mechanism for negative plant-soil feedbacks? New Phytologist 205:1195-1210.

McDonald, D., J. C. Clemente, J. Kuczynski, J. R. Rideout, J. Stombaugh, D. Wendel, A. Wilke, S. Huse, J. Hufnagle, F. Meyer, R. Knight, and J. G. Caporaso. 2012. The Biological Observation Matrix (BIOM) format or: how I learned to stop worrying and love the ome-ome. GigaScience 1:7.

Meisner, A., E. Bååth, and J. Rousk. 2013a. Microbial growth responses upon rewetting soil dried for four days or one year. Soil Biology and Biochemistry 66:188-192.

Meisner, A., W. de Boer, J. H. C. Cornelissen, and W. H. van der Putten. 2012. Reciprocal effects of litter from exotic and congeneric native plant species via soil nutrients. PLoS ONE 7:e31596.

Meisner, A., W. de Boer, K. J. F. Verhoeven, H. T. S. Boschker, and W. H. van der Putten. 2011. Comparison of nutrient acquisition in exotic plant species and congeneric natives. Journal of Ecology 99:1308-1315.

Meisner, A., G. B. De Deyn, W. de Boer, and W. H. van der Putten. 2013b. Soil biotic legacy effects of extreme weather events influence plant invasiveness. Proceedings of the National Academy of Sciences 110:9835-9838.

Meisner, A., S. Jacquiod, B. L. Snoek, F. C. ten Hooven, and W. H. van der Putten. 2018. Drought Legacy Effects on the Composition of Soil Fungal and Prokaryote Communities. Frontiers in Microbiology 9.

Meisner, A., A. Leizeaga, J. Rousk, and E. Bååth. 2017. Partial drying accelerates bacterial growth recovery to rewetting. Soil Biology and Biochemistry 112:269-276.

Mendes, R., P. Garbeva, and J. M. Raaijmakers. 2013. The rhizosphere microbiome: significance of plant beneficial, plant pathogenic, and human pathogenic microorganisms. FEMS Microbiology Reviews 37:634-663.

Mitchell, C. E., and A. G. Power. 2003. Release of invasive plants from fungal and viral pathogens. Nature 421:625-627.

Morriën, E., H. Duyts, and W. H. v. d. Putten. 2012. Effects of native and exotic range-expanding plant species on taxonomic and functional composition of nematodes in the soil food web. Oikos 121:181-190.

Morriën, E., T. Engelkes, M. Macel, A. Meisner, and W. H. van der Putten. 2010. Climate change and invasion by intracontinental range-expanding exotic plants: the role of biotic interactions. Ann Bot 105:843-848.

Morriën, E., W. H. van der Putten, and N. Wurzburger. 2013. Soil microbial community structure of range-expanding plant species differs from co-occurring natives. Journal of Ecology 101:1093-1102.

NDFF. 2018. Verspreidingsatlas planten. Nationale Databank Flora en Fauna.

Nguyen, N. H., Z. Song, S. T. Bates, S. Branco, L. Tedersoo, J. Menke, J. S. Schilling, and P. G. Kennedy. 2016. FUNGuild: An open annotation tool for parsing fungal community datasets by ecological guild. Fungal Ecology 20:241-248. 
Oksanen, J., F. G. Blanchet, M. Friendly, R. Kindt, P. Legendre, D. McGlinn, P. R. Minchin, R. B. O'Hara, G. L. Simpson, P. Solymos, M. H. H. Stevens, E. Szoecs, and H. Wagner. 2018. The vegan package.

Ordonez, A., I. J. Wright, and H. Olff. 2010. Functional differences between native and alien species: a global-scale comparison. Functional Ecology 24:1353-1361.

Palta, J. A., and P. J. Gregory. 1997. Drought affects the fluxes of carbon to roots and soil in $13 \mathrm{C}$ pulse-labelled plants of wheat. Soil Biology and Biochemistry 29:1395-1403.

Parmesan, C., and G. Yohe. 2003. A globally coherent fingerprint of climate change impacts across natural systems. Nature 421:37-42.

Paterson, E., T. Gebbing, C. Abel, A. Sim, and G. Telfer. 2007. Rhizodeposition shapes rhizosphere microbial community structure in organic soil. New Phytologist 173:600-610.

Pauli, H., M. Gottfried, S. Dullinger, O. Abdaladze, M. Akhalkatsi, J. L. B. Alonso, G. Coldea, J. Dick, B. Erschbamer, R. F. Calzado, D. Ghosn, J. I. Holten, R. Kanka, G. Kazakis, J. Kollar, P. Larsson, P. Moiseev, D. Moiseev, U. Molau, J. M. Mesa, L. Nagy, G. Pelino, M. Puscas, G. Rossi, A. Stanisci, A. O. Syverhuset, J. P. Theurillat, M. Tomaselli, P. Unterluggauer, L. Villar, P. Vittoz, and G. Grabherr. 2012. Recent plant diversity changes on Europe's mountain summits. Science 336:353-355.

Peñuelas, J., and I. Filella. 2001. Responses to a Warming World. Science 294:793-795.

Peñuelas, J., P. Prieto, C. Beier, C. Cesaraccio, P. De Angelis, G. De Dato, B. A. Emmett, M. Estiarte, J. Garadnai, A. Gorissen, E. K. Lang, G. Kroel-Dulay, L. Llorens, G. Pellizzaro, T. Riis-Nielsen, I. K. Schmidt, C. Sirca, A. Sowerby, D. Spano, and A. Tietema. 2007. Response of plant species richness and primary productivity in shrublands along a north-south gradient in Europe to seven years of experimental warming and drought: reductions in primary productivity in the heat and drought year of 2003. Global Change Biology 13:2563-2581.

Philippot, L., J. M. Raaijmakers, P. Lemanceau, and W. H. van der Putten. 2013. Going back to the roots: the microbial ecology of the rhizosphere. Nat Rev Microbiol 11:789-799.

Pimm, S. L. 1984. The complexity and stability of ecosystems. Nature 307:321.

Preece, C., and J. Peñuelas. 2016. Rhizodeposition under drought and consequences for soil communities and ecosystem resilience. Plant and Soil 409:1-17.

Pyšek, P., V. Jarošík, P. E. Hulme, J. Pergl, M. Hejda, U. Schaffner, and M. Vilà. 2012. A global assessment of invasive plant impacts on resident species, communities and ecosystems: the interaction of impact measures, invading species' traits and environment. Global Change Biology 18:1725-1737.

Pyšek, P., D. M. Richardson, J. Pergl, V. Jarošík, Z. Sixtová, and E. Weber. 2008. Geographical and taxonomic biases in invasion ecology. Trends in Ecology \& Evolution 23:237-244.

R Core Team. 2017. R: A language and environment for statistical computing. R Foundation for Statistical Computing, Vienna, Austria.

Raaijmakers, J. M., T. C. Paulitz, C. Steinberg, C. Alabouvette, and Y. Moënne-Loccoz. 2009. The rhizosphere: a playground and battlefield for soilborne pathogens and beneficial microorganisms. Plant and Soil 321:341-361.

Reinhart, K. O., A. Packer, W. H. van der Putten, and K. Clay. 2003. Plant-soil biota interactions and spatial distribution of black cherry in its native and invasive ranges. Ecology Letters 6:10461050.

Reinhart, K. O., T. Tytgat, W. H. van der Putten, and K. Clay. 2010. Virulence of soil-borne pathogens and invasion by Prunus serotina. New Phytologist 186:484-495.

Rejmanek, M., and D. M. Richardson. 1996. What Attributes Make Some Plant Species More Invasive? Ecology 77:1655-1661.

Rognes, T., T. Flouri, B. Nichols, C. Quince, and F. Mahé. 2016. VSEARCH: a versatile open source tool for metagenomics. PeerJ Preprints 4:e2409v2401.

Rothstein, D. E., P. M. Vitousek, and B. L. Simmons. 2004. An Exotic Tree Alters Decomposition and Nutrient Cycling in A Hawaiian Montane Forest. Ecosystems 7:805-814. 
Rustad, L., J. Campbell, G. Marion, R. Norby, M. Mitchell, A. Hartley, J. Cornelissen, and J. Gurevitch. 2001. A meta-analysis of the response of soil respiration, net nitrogen mineralization, and aboveground plant growth to experimental ecosystem warming. Oecologia 126:543-562.

Sanaullah, M., E. Blagodatskaya, A. Chabbi, C. Rumpel, and Y. Kuzyakov. 2011. Drought effects on microbial biomass and enzyme activities in the rhizosphere of grasses depend on plant community composition. Applied Soil Ecology 48:38-44.

Sardans, J., M. Bartrons, O. Margalef, A. Gargallo-Garriga, I. A. Janssens, P. Ciais, M. Obersteiner, B. D. Sigurdsson, H. Y. H. Chen, and J. Peñuelas. 2017. Plant invasion is associated with higher plant-soil nutrient concentrations in nutrient-poor environments. Global Change Biology 23:1282-1291.

Sardans, J., and J. Peñuelas. 2012. The Role of Plants in the Effects of Global Change on Nutrient Availability and Stoichiometry in the Plant-Soil System. Plant Physiology 160:1741-1761.

Sayer, E. J., M. S. Heard, H. K. Grant, T. R. Marthews, and E. V. J. Tanner. 2011. Soil carbon release enhanced by increased tropical forest litterfall. Nature Climate Change 1:304.

Sayer, E. J., A. E. Oliver, J. D. Fridley, A. P. Askew, R. T. E. Mills, and J. P. Grime. 2017. Links between soil microbial communities and plant traits in a species-rich grassland under long-term climate change. Ecology and Evolution 7:855-862.

Scharfy, D., H. Eggenschwiler, H. Olde Venterink, P. J. Edwards, and S. Güsewell. 2009. The invasive alien plant species Solidago gigantea alters ecosystem properties across habitats with differing fertility. Journal of Vegetation Science 20:1072-1085.

Scharfy, D., A. Funk, H. Olde Venterink, and S. Güsewell. 2011. Invasive forbs differ functionally from native graminoids, but are similar to native forbs. New Phytologist 189:818-828.

Schimel, J., T. C. Balser, and M. Wallenstein. 2007. Microbial stress-response physiology and its implications for ecosystem function. Ecology 88:1386-1394.

Schindlbacher, A., S. Wunderlich, W. Borken, B. Kitzler, S. Zechmeister-Boltenstern, and R. Jandl. 2012. Soil respiration under climate change: prolonged summer drought offsets soil warming effects. Global Change Biology 18:2270-2279.

Setälä, H., and M. A. McLean. 2004. Decomposition rate of organic substrates in relation to the species diversity of soil saprophytic fungi. Oecologia 139:98-107.

Singh, B. K., P. Millard, A. S. Whiteley, and J. C. Murrell. 2004. Unravelling rhizosphere-microbial interactions: opportunities and limitations. Trends in Microbiology 12:386-393.

Stark, J. M., and M. K. Firestone. 1995. Mechanisms for soil moisture effects on activity of nitrifying bacteria. Applied and Environmental Microbiology 61:218-221.

Stefanowicz, A. M., M. Stanek, M. Nobis, and S. Zubek. 2016. Species-specific effects of plant invasions on activity, biomass, and composition of soil microbial communities. Biology and Fertility of Soils 52:841-852.

Strauss, S. Y., C. O. Webb, and N. Salamin. 2006. Exotic taxa less related to native species are more invasive. Proc Natl Acad Sci U S A 103:5841-5845.

Stursova, M., and R. L. Sinsabaugh. 2008. Stabilization of oxidative enzymes in desert soil may limit organic matter accumulation. Soil Biology and Biochemistry 40:550-553.

Suding, K. N., W. Stanley Harpole, T. Fukami, A. Kulmatiski, A. S. MacDougall, C. Stein, W. H. van der Putten, and M. Hutchings. 2013. Consequences of plant-soil feedbacks in invasion. Journal of Ecology 101:298-308.

Tamis, W. L. M., M. V. t. Zelfde, R. V. Meijden, and H. A. U. Haes. 2005. Changes in Vascular Plant Biodiversity in the Netherlands in the 20th Century Explained by their Climatic and other Environmental Characteristics. Climatic Change 72:37-56.

te Beest, M., J. J. Le Roux, D. M. Richardson, A. K. Brysting, J. Suda, M. Kubešová, and P. Pyšek. 2012. The more the better? The role of polyploidy in facilitating plant invasions. Annals of Botany 109:19-45.

te Beest, M., N. Stevens, H. Olff, and W. H. van der Putten. 2009. Plant-soil feedback induces shifts in biomass allocation in the invasive plant Chromolaena odorata. Journal of Ecology 97:12811290. 
Tedersoo, L., M. Bahram, S. Põlme, U. Kõljalg, N. S. Yorou, R. Wijesundera, L. V. Ruiz, A. M. VascoPalacios, P. Q. Thu, A. Suija, M. E. Smith, C. Sharp, E. Saluveer, A. Saitta, M. Rosas, T. Riit, D. Ratkowsky, K. Pritsch, K. Põldmaa, M. Piepenbring, C. Phosri, M. Peterson, K. Parts, K. Pärtel, E. Otsing, E. Nouhra, A. L. Njouonkou, R. H. Nilsson, L. N. Morgado, J. Mayor, T. W. May, L. Majuakim, D. J. Lodge, S. S. Lee, K.-H. Larsson, P. Kohout, K. Hosaka, I. Hiiesalu, T. W. Henkel, H. Harend, L.-d. Guo, A. Greslebin, G. Grelet, J. Geml, G. Gates, W. Dunstan, C. Dunk, R. Drenkhan, J. Dearnaley, A. De Kesel, T. Dang, X. Chen, F. Buegger, F. Q. Brearley, G. Bonito, S. Anslan, S. Abell, and K. Abarenkov. 2014. Global diversity and geography of soil fungi. Science 346.

Ter Braak, C., and P. Šmilauer. 2012. Canoco reference manual and user's guide: software for ordination, version 5.0. Microcomputer Power, Ithaca, USA, 496 pp.

Thomas, C. D., A. Cameron, R. E. Green, M. Bakkenes, L. J. Beaumont, Y. C. Collingham, B. F. N. Erasmus, M. F. de Siqueira, A. Grainger, L. Hannah, L. Hughes, B. Huntley, A. S. van Jaarsveld, G. F. Midgley, L. Miles, M. A. Ortega-Huerta, A. Townsend Peterson, O. L. Phillips, and S. E. Williams. 2004. Extinction risk from climate change. Nature 427:145.

Tian, K., X. Kong, J. Gao, Y. Jia, H. Lin, Z. He, Y. Ji, Z. Bei, and X. Tian. 2018. Local root status: a neglected bio-factor that regulates the home-field advantage of leaf litter decomposition. Plant and Soil.

Toberman, H., C. Freeman, C. Evans, N. Fenner, and R. R. E. Artz. 2008. Summer drought decreases soil fungal diversity and associated phenol oxidase activity in upland Calluna heathland soil. FEMS Microbiology Ecology 66:426-436.

Valliere, J. M., and E. B. Allen. 2016. Nitrogen enrichment contributes to positive responses to soil microbial communities in three invasive plant species. Biological Invasions 18:2349-2364.

van de Voorde, T. F. J., W. H. van der Putten, and T. M. Bezemer. 2012. Soil inoculation method determines the strength of plant-soil interactions. Soil Biology and Biochemistry 55:1-6.

van der Heijden, M. G., R. D. Bardgett, and N. M. van Straalen. 2008. The unseen majority: soil microbes as drivers of plant diversity and productivity in terrestrial ecosystems. Ecol Lett 11:296-310.

van der Putten, W. H. 2012. Climate Change, Aboveground-Belowground Interactions, and Species' Range Shifts. Annual Review of Ecology, Evolution, and Systematics 43:365-383.

van der Putten, W. H., R. D. Bardgett, J. D. Bever, T. M. Bezemer, B. B. Casper, T. Fukami, P. Kardol, J. N. Klironomos, A. Kulmatiski, J. A. Schweitzer, K. N. Suding, T. F. J. Van de Voorde, D. A. Wardle, and M. Hutchings. 2013. Plant-soil feedbacks: the past, the present and future challenges. Journal of Ecology 101:265-276.

van der Putten, W. H., M. A. Bradford, E. P. Brinkman, T. F. J. Voorde, and G. F. C. Veen. 2016. Where, when and how plant-soil feedback matters in a changing world. Functional Ecology 30:11091121.

van der Putten, W. H., M. Macel, and M. E. Visser. 2010. Predicting species distribution and abundance responses to climate change: why it is essential to include biotic interactions across trophic levels. Philos Trans R Soc Lond B Biol Sci 365:2025-2034.

van Grunsven, R. H. A., W. H. van der Putten, T. M. Bezemer, W. L. M. Tamis, F. Berendse, and E. M. Veenendaal. 2007. Reduced plant-soil feedback of plant species expanding their range as compared to natives. Journal of Ecology 95:1050-1057.

Van Grunsven, R. H. A., W. H. van der Putten, T. Martijn Bezemer, F. Berendse, and E. M. Veenendaal. 2010. Plant-soil interactions in the expansion and native range of a poleward shifting plant species. Global Change Biology 16:380-385.

van Kleunen, M., E. Weber, and M. Fischer. 2010. A meta-analysis of trait differences between invasive and non-invasive plant species. Ecol Lett 13:235-245.

Van Nuland, M. E., J. K. Bailey, and J. A. Schweitzer. 2017. Divergent plant-soil feedbacks could alter future elevation ranges and ecosystem dynamics. Nature Ecology \& Evolution 1:0150.

Veen, G. F., G. T. Freschet, A. Ordonez, and D. A. Wardle. 2015a. Litter quality and environmental controls of home-field advantage effects on litter decomposition. Oikos 124:187-195. 
Veen, G. F. C., M. K. Sundqvist, D. A. Wardle, and M. J. Briones. 2015b. Environmental factors and traits that drive plant litter decomposition do not determine home-field advantage effects. Functional Ecology 29:981-991.

Verhoeven, K. J., A. Biere, J. A. Harvey, and W. H. van der Putten. 2009. Plant invaders and their novel natural enemies: who is naive? Ecol Lett 12:107-117.

Vilà, M., J. L. Espinar, M. Hejda, P. E. Hulme, V. Jarosik, J. L. Maron, J. Pergl, U. Schaffner, Y. Sun, and P. Pysek. 2011. Ecological impacts of invasive alien plants: a meta-analysis of their effects on species, communities and ecosystems. Ecol Lett 14:702-708.

Vitousek, P. M., H. A. Mooney, J. Lubchenco, and J. M. Melillo. 1997. Human Domination of Earth's Ecosystems. Science 277:494-499.

Waldrop, M. P., and M. K. Firestone. 2006a. Response of microbial community composition and function to soil climate change. Microb Ecol 52:716-724.

Waldrop, M. P., and M. K. Firestone. 2006b. Seasonal Dynamics of Microbial Community Composition and Function in Oak Canopy and Open Grassland Soils. Microbial Ecology 52:470-479.

Walker, T. S., H. P. Bais, E. Grotewold, and J. M. Vivanco. 2003. Root Exudation and Rhizosphere Biology. Plant Physiology 132:44-51.

Wardle, D. A., R. D. Bardgett, J. N. Klironomos, H. Setala, W. H. van der Putten, and D. H. Wall. 2004. Ecological linkages between aboveground and belowground biota. Science 304:1629-1633.

Wardle, D. A., and D. A. Peltzer. 2017. Impacts of invasive biota in forest ecosystems in an aboveground-belowground context. Biological Invasions 19:3301-3316.

Wertz, S., V. Degrange, J. I. Prosser, F. Poly, C. Commeaux, T. Freitag, N. Guillaumaud, and X. L. Roux. 2006. Maintenance of soil functioning following erosion of microbial diversity. Environmental Microbiology 8:2162-2169.

Wertz, S., V. Degrange, J. I. Prosser, F. Poly, C. Commeaux, N. Guillaumaud, and X. Le Roux. 2007. Decline of soil microbial diversity does not influence the resistance and resilience of key soil microbial functional groups following a model disturbance. Environmental Microbiology 9:2211-2219.

Whitaker, B. K., J. T. Bauer, J. D. Bever, and K. Clay. 2017. Negative plant-phyllosphere feedbacks in native Asteraceae hosts - a novel extension of the plant-soil feedback framework. Ecology Letters 20:1064-1073.

Williamson, M., and A. Fitter. 1996. The Varying Success of Invaders. Ecology 77:1661-1666.

Wilschut, R. A., J. C. P. Silva, P. Garbeva, and W. H. van der Putten. 2017. Belowground PlantHerbivore Interactions Vary among Climate-Driven Range-Expanding Plant Species with Different Degrees of Novel Chemistry. Frontiers in Plant Science 8.

Wolfe, B. E., and J. N. Klironomos. 2005. Breaking new ground: soil communities and exotic plant invasion. BioScience 55:477-487.

Worrich, A., H. Stryhanyuk, N. Musat, S. König, T. Banitz, F. Centler, K. Frank, M. Thullner, H. Harms, H.-H. Richnow, A. Miltner, M. Kästner, and L. Y. Wick. 2017. Mycelium-mediated transfer of water and nutrients stimulates bacterial activity in dry and oligotrophic environments. Nature Communications 8:15472.

Zhang, L., X. Ma, H. Wang, S. Liu, E. Siemann, and J. Zou. 2016a. Soil Respiration and Litter Decomposition Increased Following Perennial Forb Invasion into an Annual Grassland. Pedosphere 26:567-576.

Zhang, N., W. H. van der Putten, and G. F. Veen. 2016b. Effects of root decomposition on plant-soil feedback of early- and mid-successional plant species. New Phytologist 212:220-231. 


\section{Summary}

The disproportionate rise in greenhouse gas emissions over the last century, originating largely from anthropogenic activities, has triggered an increase of global mean temperatures. Current climate warming is occurring at an unprecedented rapid rate, with important consequences for many plant and animal species. In order to persist, plants may adapt to the warmer conditions or expand their geographical range to areas of higher latitudes and altitudes within continents, where they were previously not present. Thus, in Europe plant species from warmer climate areas, mainly from the Mediterrenean region, are currently increasing in number and abundance in Central and Northern Europe, including the Netherlands. It has been suggested that among plant species that expand their range there may be species with the potential to become invasive. Yet, relatively little is known about the ecological consequences of plant range expansion for the invaded ecosystems.

Unraveling plant-soil interactions in the context of plant range expansions may elucidate mechanisms explaining establishment success and consequences of range-expanding plant species. As range-expanding plants establish in ecosystems outside their original range, they may encounter novel soil communities. Missing specialist enemies may benefit the establishment of range-expanding plant species in the new range. However, relatively less is known about the interactions between range-expanding plant species and the bulk of the soil community, including decomposer communities. In my thesis, I studied plantsoil interactions of plants that expand their range enabled by climate warming. I focused on indirect plant-soil interactions mediated by the resources that plants provide to the soil in the form of litter or root exudates, which influence the activity of the soil decomposer community and soil nutrient availability. I compared these interactions of rangeexpanding plants to those of native plants species in order to identify the functional consequences of plant range expansion in the new range. In my approach, I mainly used phylogenetic controlled comparisons because they allowed to test the effect of plant origin and disentangling it from the effect of trait differences.

In the first part of the thesis, I determined concentrations of major nutrients $(C, N$ and $P)$ in plant tissues and nutrient availability in the soils of range-expanding plant species and natives. All parameters were measured in samples from individual plants and their associated soils in the field of the expansion (north) and the original (south) ranges. I expected that range expansion, similarly to successful exotic species, is associated with 
higher nutrients in plants and soils compared to native species. I show that differences in plant and soil chemistry of range-expanding and related native plant species were linked to plant species identity rather than origin. Furthermore, plant and soil chemistry varied between the north and south ranges but the direction and size of these latitudinal differences was dependent on the parameter and plant species considered. Therefore, I concluded that there are no general differences in plant and soil nutrients between rangeexpanding and congeneric native plant species that co-occur with the range expanders in the novel range. Thus, I found no evidence that patterns observed in the literature on invasive exotic species apply to plant species that expand their range within continents.

Then, in a controlled greenhouse pot experiment, I studied the functional consequences of plant origin by comparing rhizosphere bacterial communities and soil community functioning of range-expanding and native plant species. Overall, exotic plant species have been shown to alter soil microbial communities and their functions. Thus, I expected that range-expanding plant species would have similar influence on the soil communities in their new range, where they are novel. I grew plants for three months and examined four replicate plant genera to determine general effects of range-expanding plant species. At the end of each month, I destructively sampled part of the experiment and assessed bacterial communities with $16 \mathrm{~S}$ sequencing, and community level functioning by measuring enzyme activity and the respiration response of the soil communities to organic substrates of varying recalcitrance. To disentangle the effects of plant origin from those of the specific field sites where these plants currently occur, I grew plants in soils collected from underneath the same plant species in the field and in soils that are novel to them. The main finding of this experiment was that, when controlling for both species relatedness and soil characteristics, range-expanding plant species influence soil bacterial community composition and nutrient cycling in a manner similar to congeneric related native species. These results also illustrate the importance of phylogenetically controlled comparisons to disentangle plant origin effects from effects of contrasting plant traits.

As a follow up, I studied feedback effects from the rhizosphere of range-expanding and native plants species, as well as feedback effects induced by decomposing plant litter and their combination. In the literature, feedback effects of plant litter have been less studied than those mediated by the rhizosphere of living plants. Nevertheless, plant litter feedbacks have been suggested to promote the performance of exotic plant species in the new range. Therefore, in this two-phase feedback experiment, I conditioned the soil with living plants, plant litter and their combination. I took a sensitive approach using control 
soils from the congeneric species in order to determine whether specialist soil biota may be lost upon establishment in the new range. Here, I expected less negative feedback to range-expanders than to natives in soils conditioned by living plants and that all plant species would benefit from soils conditioned by the litter of range-expanders as litter of range-expanders is expected to have higher nutrient concentrations than the litter of natives. In this experiment, I tested plant-soil feedbacks on subsequent plant biomass and on litter decomposition. Results indicate that both range-expanding and native plant species developed neutral plant-soil feedbacks in both the rhizosphere and litter conditioned soils. However, two out of the four examined range-expanders produced more biomass than the natives in soils conditioned by litter. Furthermore, litter decomposition was also higher for range-expanders than natives in two out of four genera, but decomposition was unaffected by soil conditioning. Overall, the experimental setup used provided conservative plant-soil feedback effects both to plant growth and decomposition, indicating similar roles of specialist soil communities for range-expanding and native plant species under these experimental conditions. For the specific case of Centaurea, I show that it produces more biomass under high soil nutrient availability and it decomposes more than its congeneric native. Therefore, I conclude that in some specific cases, range-expanders may benefit from high nutrient availability, which may also be induced by litter feedbacks.

Plant range expansion may interact with direct effects of climate change, such as drought events, in the new range. In Chapter 5, I investigated the role of range-expanding plant communities and soil biota from the original range on driving soil functioning during and after drought. Because plant species and soils that originate from dryer areas are expected to cope better with drought, I hypothesized that soils of range-expanding plant communities would be less affected by drought and recovered faster than soils of native plant communities, especially when soils were inoculated with live soil from the original range. In a mesocosm experiment outside, plant communities of range-expanding and native plant species were established in soils with and without inocula from the original range. I measured soil functions (litter decomposition, carbon mineralization and enzyme activities), microbial biomass and the relative abundance of soil saprophytic fungi immediately after drought and at 6 and 12 weeks after rewetting. While drought affected most soil functions equally, after re-wetting, soils of native plant communities with a history of drought had more litter mass loss and higher relative abundance of saprophytic fungi than soils without drought and soils of range expanders. This was not the case in the 
soils of range-expanders, where soil functions were not stimulated over the watered controls after drought and regardless of soil inocula. Overall, soil inocula effects were weak and all soil functions were recovered by the end of the experiment. These results suggest that shortly after soil re-wetting, functional consequences of plant range expansion may interact with the effects of drought.

With this research, I contributed to the understanding of the interactions that rangeexpanding plants establish with soils in the new range and of the specific role of plant origin in these interactions. I have shown that overall range-expanding plant species examined do not resemble invasive exotic species because they did not affect rhizosphere bacterial communities and soil functions differently from related natives. Thereby, I have found little evidence suggesting that, in general, range-expanding plant species may impact soil communities and functions similarly to invasive exotic species. Nevertheless, in some specific cases range-expanding plant species may benefit from high nutrient availability in the soil and positive litter decomposition feedbacks. Finally, I have shown that plant-soil interactions of range-expanding plants may differ most from natives under climate extreme events such as drought. To address remaining questions on the impact of indirect plant-soil interactions for range-expanders, field studies should incorporate plantsoil feedbacks of plant litter. Furthermore, experiments may assess potential benefits for range-expanders over natives in competition. Also, it is crucial that further studies on examining ecosystem functioning under climate change scenarios incorporate multifactorial designs accounting for changes in plant community composition induced by climate change. 


\section{Sumari}

L'increment desproporcionat de les emissions de gasos d'efecte hivernacle, originades majoritàriament de les activitats antropogèniques, ha desencadenat un augment de la temperatura mitjana global. Aquest escalfament s'està produint a una velocitat sense precedents, i té importants conseqüències per a nombroses espècies vegetals i animals. Per tal de persistir en aquestes condicions climàtiques canviants, les plantes s'han d'adaptar o han d'incrementar el seu rang de distribució cap a latituds i altituds més elevades. A Europa, plantes que són natives de zones de clima càlid, majoritàriament de la zona Mediterrània, estan incrementant en nombre i abundància al centre i nord del continent, incloent els Països Baixos. Estudis previs han suggerit que, entre les plantes que incrementen el seu rang de distribució, poden haver-hi espècies potencialment invasores. Tot i així, en termes generals es desconeixen quins són els impactes ecològics de les expansions de plantes per als ecosistemes on arriben a establir-se.

Les plantes interaccionen contínuament amb els organismes del sòl, i el seu creixement i desenvolupament depèn en gran mesura d'aquestes interaccions. Les interaccions plantasòl poden tenir un paper important durant les expansions d'espècies vegetals, i poden ajudar a identificar els mecanismes que determinen l'èxit i l'impacte d'aquestes plantes. Quan les plantes s'instal-len fora del seu rang de distribució original, poden establir interaccions amb organismes del sòl diferents de les que tenien en el seu rang original. Això és degut al fet que els organismes del sòl solen tenir distribucions geogràfiques diferents a les de les plantes, o al fet que responen d'una manera diferent a l'escalfament global i, per tant, no varien el seu rang de distribució o ho fan a velocitats molt inferiors a les de les plantes, ja que es dispersen més lentament. Quan això passa, per exemple, les plantes en expansió poden deixar enrere organismes patògens que es troben entre els organismes del sòl. D'aquesta manera les plantes en expansió poden beneficiar-se d'una comunitat del sòl lliure de patògens. A banda de les interaccions planta-patògen, però, es desconeixen les interaccions que s'estableixen entre les plantes i les comunitats microbianes del sòl que s'encarreguen de descompondre la matèria orgànica i del reciclatge de nutrients, i que constitueixen la major part dels microorganismes del sòl. Per tant, l'objectiu d'aquesta tesi és estudiar les interaccions que s'estableixen entre les plantes que estan ampliant el seu rang i els microorganismes del sòl responsables de la descomposició de la matèria orgànica i del reciclatge de nutrients. En general, les plantes proveeixen de recursos als microorganismes del sòl en forma de fullaraca, material orgànic i exsudats de les arrels, que aquests utilitzen com a font de matèria i energia, per tant, 
modulen l'activitat microbiana del sòl. Quan aquests recursos provenen de plantes exòtiques, és a dir, plantes que són noves a l'ecosistema, es poden produir alteracions en la composició i funcionament de les comunitats del sòl. Aquestes alteracions poden repercutir de manera indirecta al creixement de les plantes a través de la mineralització i la disponibilitat de nutrients. En els diferents capítols d'aquesta tesi, he comparat les interaccions planta-sòl de les plantes en expansió que s'estan establint als Països Baixos amb les de les plantes que són natives per tal d'identificar les conseqüències de les expansions de plantes pel funcionament dels ecosistemes on s'estableixen. En el meu procediment, he utilitzat comparacions d'espècies que estan relacionades filogenèticament, les quals m'han permès determinar els efectes de l'origen de les plantes (natives o en expansió) i separar-los d'aquells efectes que estan condicionats per altres trets propis de cada espècie.

Primerament, vaig determinar les concentracions de carboni i nutrients als teixits de les plantes i la disponibilitat de nutrients als sòls on creixen aquestes plantes al camp. Vaig prendre mostres de fulles i sòls que pertanyen a plantes en expansió i a plantes natives. La meva hipòtesi principal afirmava que les plantes en expansió estan associades amb una concentració més elevada de nutrients al teixit vegetal i al sòl que les plantes natives, tal i com passa amb les plantes exòtiques que aconsegueixen establir-se de manera exitosa fora del seu rang original. Els resultats obtinguts mostren que les diferències observades depenen de cada espècie individualment, però no hi ha una tendència general causada per l'origen geogràfic de les plantes. Per tant, vaig concloure que no hi ha diferències generals entre les plantes en expansió i les natives pel que fa al contingut de nutrients de les plantes i dels sòls.

Seguidament vaig estudiar els efectes que tenen les plantes sobre les comunitats de la rizosfera. Vaig comparar les comunitats bacterianes i l'activitat de la comunitat microbiana a la rizosfera de plantes en expansió i plantes natives. En general, es coneix que les plantes exòtiques que s'estableixen de manera exitosa fora del seu rang modifiquen les comunitats bacterianes que habiten la rizosfera i n'alteren així el seu funcionament. Per tant, la hipòtesi era que les plantes que han incrementat el seu rang poden tenir efectes similars al de les plantes exòtiques en el seu nou rang, ja que hi són espècies noves. Vaig investigar quatre gèneres de plantes diferents, que vaig utilitzar com a rèpliques per tal d'identificar un efecte general de les plantes que amplien la seva distribució. Durant el desenvolupament de les plantes, vaig recollir mostres de sòl de la rizosfera, vaig determinar la comunitat bacteriana utilitzant tècniques de seqüenciació 16S, l'activitat 
enzimàtica i la mineralització de diversos compostos orgànics. Per tal de poder separar els efectes de l'origen de les plantes d'aquells que són causats per altres condicions dels llocs on aquestes plantes habiten en el camp, vaig fer créixer les plantes en sòls provinents de llocs on aquestes plantes són presents en el camp i en sòls als quals les plantes no han estat exposades prèviament. El resultat més important d'aquest experiment va ser que, quan es té en compte i s'eliminen els efectes de les característiques prèvies dels sòls i la relació filogenètica de les plantes a comparar, les plantes en expansió influencien la composició de les comunitats bacterianes i el reciclatge de nutrients d'una manera similar a les plantes natives. Aquests resultats remarquen la importància de les comparacions controlades filogenèticament per tal de separar els efectes causats per l'origen de les plantes dels causats per altres trets propis de cada espècie.

A continuació, vaig investigar els efectes "feedback" que s'estableixen entre les plantes i els sòls on creixen. Els efectes "feedback" són els efectes que les plantes tenen sobre el seu propi creixement o el creixement de les espècies que ocuparan el seu lloc en futures generacions a través dels canvis que les mateixes plantes indueixen mentre hi creixen. Aquests canvis són tant a nivell de les comunitats microbianes del sòl com a nivell de disponibilitat de nutrients i poden ser causats per les plantes mentre viuen o per la seva fullaraca en finalitzar el seu cicle. Els efectes "feedback" determinats per la descomposició de la fullaraca es coneixen relativament poc comparat amb els efectes originats per les plantes durant el seu creixement. Per tant, en aquest experiment, vaig estudiar aquests efectes "feedback" en el context de les plantes que amplien el seu rang, ja que s'ha vist que en alguns casos poden estimular el creixement de les plantes exòtiques. Els resultats han indicat que tant les plantes natives com les plantes en expansió tenen efectes "feedback" neutrals. Això passa tant en sòls que han estat exposats a les plantes com en sòls que han rebut la seva fullaraca. No obstant això, dues de les quatre espècies en expansió analitzades presentaven un creixement superior que les natives en sòls que havien estat condicionats amb fullaraca i, per tant, tenien una disponibilitat més alta de nutrients. També vaig observar que la descomposició de la fullaraca de les plantes en expansió era superior a la descomposició de la fullaraca de plantes natives en dues dels quatre espècies analitzades. Per tant, certes espècies de plantes en expansió poden beneficiar-se d'una disponibilitat elevada de nutrients al sòl, que pot ésser a la vegada induïda per la descomposició de la seva pròpia fullaraca.

Les conseqüències del canvi climàtic pels ecosistemes naturals son múltiples. L'expansió de la distribució de les plantes i el conseqüent canvi en la composició de les comunitats 
vegetals n'és una. A la vegada però, aquesta interacciona amb altres conseqüències més directes del canvi climàtic com, per exemple, els episodis de sequera. Les plantes típiques de climes més secs tenen més facilitat de persistir durant episodis de sequera i, per tant, seria d'esperar que les funcions del sòl on creixen també es veurien menys afectades. Això és precisament el que vaig estudiar en l'últim estudi d'aquesta tesi. Per fer-ho, vaig establir un experiment amb comunitats vegetals formades per plantes que amplien el seu rang i plantes natives i vaig determinar diferents funcions del sòl (descomposició de la matèria orgànica, mineralització de carboni i activitat enzimàtica), la biomassa microbiana i l'abundància relativa de fongs saprofítics. Tots els paràmetres van ser mesurats al final d'un episodi de sequera, i al cap de 6 i 12 setmanes després de tornar a regar. La sequera va fer disminuir les funcions del sòl, sense importar el tipus de comunitat vegetal. Per altra banda, després de regar, la descomposició de matèria orgànica i l'abundància relativa de fongs saprofítics en el sòls que havien estat exposats a la sequera van incrementar per sobre del tractament control, però només en sòls de plantes natives. En el cas dels sòls de plantes que amplien el seu rang, les funcions es van recuperar lentament però en cap cas van ser estimulades per sobre del llindar del tractament control. Tot i que les funcions dels sòls que van patir sequera van ser recuperades al final de l'experiment, aquest resultats suggereixen que cal tenir en compte les interaccions dels diferents factors del canvi climàtic, sobretot a curt termini i en escenaris de canvi climàtic on els episodis de sequera poden ser més recurrents.

La recerca d'aquesta tesi ha contribuït a entendre les interaccions planta-sòl en el context de les expansions de plantes, i a determinar el rol de l'origen geogràfic de les plantes en aquestes interaccions. He demostrat que les espècies de plantes en expansió que hem examinat no s'assemblen a les plantes exòtiques que són invasores, ja que la seva composició química, els seus efectes sobre les comunitats i funcions dels sòls no es diferencien dels de les plantes natives. Tanmateix, he mostrat que certes espècies de plantes en expansió, sense ser la tendència general, poden beneficiar-se d'una disponibilitat de nutrients al sòl elevada i poden tenir un efecte "feedback" positiu sobre el seu propi creixement a través de la descomposició de la seva fullaraca. Finalment, he demostrat que durant fenòmens meteorològics extrems com, per exemple episodis de sequera, les interaccions planta-sòl esdevenen més crucials per mantenir el funcionament dels ecosistemes. Per tal de continuar la investigació i resoldre les preguntes que queden pendents fruit dels resultats d'aquesta tesi, caldria portar a terme estudis de camp de més llarga durada, que incorporessin els efectes "feedback" causats per la descomposició del 
material vegetal i que incloguessin les relacions competitives entre plantes natives i plantes en expansió. També seria important que els estudis que investiguen els efectes del canvi climàtic pel funcionament dels ecosistemes incloguessin en els seus dissenys experimentals els canvis en la composició de les comunitats vegetals que alhora són induïts pel mateix canvi climàtic. 


\section{Samenvatting}

De toename in de uitstoot van broeikasgassen zorgt dat de aarde gedurende de laatste eeuw is opgewarmd. Dit heeft grote gevolgen voor planten en dieren. Om te blijven bestaan, moeten ze zich aanpassen aan de nieuwe omstandigheden, of hun areaal kunnen uitbreiden naar gebieden met meer geschikte omstandigheden. Binnen Europa heeft dit tot gevolg dat planten uit Mediterrane gebieden hun areaal uitbreiden naar Centraal- en Noord-Europa. Tussen deze planten zitten mogelijk soorten die invasief kunnen worden in het nieuwe areaal. We weten echter nog zeer weinig over de ecologische gevolgen van de areaaluitbreiding van planten voor de door hen gekoloniseerde ecosystemen.

Om het succes en de gevolgen van de vestiging van areaaluitbreidende planten te begrijpen, is het nodig om plant-bodem interacties nader te onderzoeken. In hun nieuwe leefgebied komen areaaluitbreiders in aanraking met andere bodemgemeenschappen dan in hun herkomstgebieden. Eerder onderzoek heeft aangetoond dat areaaluitbreiders in hun nieuwe leefgebied baat kunnen hebben bij het ontbreken van gespecialiseerde vijanden in de bodem. Naast het ontbreken van vijanden kunnen meer verschillen bestaan tussen het bodemleven in herkomstgebieden en de nieuwe leefgebieden. Zo kunnen areaaluitbreiders bijvoorbeeld in aanraking komen met nieuwe bodemorganismen die organisch materiaal afbreken. Er is echter weinig bekend over de gevolgen van interacties tussen areaaluitbreidende planten en organismen die organisch materiaal afbreken voor de vestiging in en het functioneren van de nieuwe leefgebieden.

In mijn proefschrift bestudeer ik de interacties tussen areaaluitbreidende planten en bodemgemeenschappen. Ik richt me met name op indirecte interacties tussen levende planten en bodemorganismen, waarbij organische stoffen zoals strooisel (dood plantenmateriaal) of wortelexudaten worden afgebroken en omgezet tot voor de planten opneembare voedingsstoffen. Ik bestudeer ook de gevolgen van de afbraak van organische stoffen voor de kringloop van koolstof en voedingsstoffen in de bodem. Ik vergelijk plantbodeminteracties tussen inheemse and areaaluitbreidende planten om de functionele gevolgen van areaaluitbreiding voor het nieuwe leefgebied te begrijpen. Ik gebruik paren van fylogenetisch verwante plantensoorten om het effect van plantenherkomst te kunnen testen, terwijl ik corrigeer voor verschillen in andere planteneigenschappen.

In het eerste deel van mijn proefschrift heb ik de concentratie van voedingsstoffen (koolstof, stikstof en fosfaat) in plantenweefsel en de beschikbaarheid van voedingsstoffen in de bodem bepaald voor inheemse en areaaluitbreidende plantensoorten. Ik heb 
monsters verzameld van individuele planten in het veld in het nieuwe leefgebied (NoordEuropa) en in het gebied waar de areaaluitbreiders inheems zijn (Zuid-Europa). Ik verwachtte dat, net als bij intercontintenale exoten, de nutrientenconcentraties hoger zouden zijn in plantenweefsel en bodems van areaaluitbreiders dan van inheemse planten. Ik vond echter dat de hoeveelheid voedingsstoffen in planten en bodem vooral verschilden tussen plantensoorten, maar niet per se gerelateerd waren aan de herkomst van de plantensoorten. Daarnaast verschilden de hoeveelheid voedingsstoffen in planten en de bodems tussen het noorden en zuiden van Europa, maar de richting en grootte van deze verschillen was afhankelijk van de plantensoort en de parameter die ik testte. Mijn conclusie is dat er geen algemene verschillen zijn in de concentratie van voedingsstoffen in plantenweefsel en bodem tussen inheemse en areaaluitbreidende plantensoorten. Patronen die eerder werden gevonden voor invasieve intercontintale exoten worden dus niet bevestigd voor areaaluitbreiders.

Vervolgens heb ik in een kasexperiment onderzocht hoe plantenherkomst de samenstelling en het functioneren van bacteriële gemeenschappen in de wortelzone beïnvloedde. Ik verwachtte dat, net als in het geval van intercontinentale exoten, de samenstelling en het functioneren van microbiële gemeenschappen anders zou zijn in de wortelzone van areaaluitbreiders dan in de wortelzone van inheemse plantensoorten. Ik heb vier paren (elk bestaande uit een areaaluitbreider en een inheemse plantensoort van hetzelfde geslacht) onderzocht. Planten groeiden in een bodem met een gemeenschap die was verzameld in het veld onder een plant van dezelfde soort, of in een bodem waar andere plantensoorten voorkwamen en die dus volledig nieuw was voor de testplanten. $\mathrm{Na}$ één, twee en drie maanden heb ik bodemmonsters uit de wortelzone van de planten genomen en met $16 \mathrm{~S}$ sequencing de samenstelling van de bacteriële gemeenschap bepaald. Daarnaast heb ik de activiteit van enzymen gemeten en de bodemrespiratie bepaald na het toedienen van diverse organische substraten. Het belangrijkste resultaat van dit experiment was dat areaaluitbreidende en inheemse plantensoorten, die in een volledige nieuwe bodem groeiden, de samenstelling en het functioneren van bodemgemeenschap op dezelde manier beïnvloedden. Dit experiment laat zien dat het belangrijk is om fylogenetisch gerelateerde soorten te gebruiken om effecten van plantenherkomst te kunnen scheiden van effecten van andere planteneigenschappen.

Daarna heb ik bestudeerd hoe veranderingen in de bodem via levende planten, strooisel en hun combinatie terugkoppelen naar plantengroei en de afbraak van strooisel. Eerdere studies over plant-bodem terugekoppelingen richtten zich vooral op effecten via levende 
planten, terwijl minder bekend is over terugkoppelingen die veroorzaakt worden door strooisel. Desalniettemin wordt verondersteld dat terugkoppelingen via strooiselafbraak de groei van exoten kunnen stimuleren. In dit experiment heb ik bodems geconditioneerd met levende planten, strooisel en de combinatie van beiden. Daarna heb ik bepaald hoe areaaluitbreiders en inheemse plantensoorten groeien in deze bodems, waarbij ik planten uit hetzelfde geslacht heb vergeleken. Ik verwachtte dat als gevolg van de aanwezigheid van gespecialiseerde vijanden de groei van inheemse plantensoorten meer geremd zou zijn in bodems die geconditioneerd waren door planten van hun eigen soort dan de groei van areaaluitbreiders. Ook verwachtte ik dat alle plantensoorten beter zouden groeien in bodems met strooisel van areaaluitbreiders dan met strooisel van inheemse soorten, omdat strooisel van areaaluitbreiders mogelijk een hogere concentratie aan voedingsstoffen bevat. Ik vond dat inheemse en areaaluitbreidende planten een neutrale plant-bodem terugkoppeling ontwikkelden, zowel via levende planten als via strooisel. Toch hadden twee van de vier areaaluitbreidende plantensoorten meer biomassa dan inheemse plantensoorten in bodems die waren geconditioneerd door strooisel. Bodemconditionering had geen effect op strooiselafbraak, maar strooisel van twee van de vier areaaluitbreiders werd sneller afgebroken dan van de inheemse plantensoorten. Mijn resultaten laten dus zien dat effecten van plant-bodemterugkoppelingen, zowel via levende planten als strooisel, gelimiteerd waren en vergelijkbaar waren voor areaaluitbreiders en nauw verwante inheemse plantensoorten. Echter, in het geval van Centaurea soorten produceerde de areaaluitbreider meer biomassa als er veel voedingsstoffen beschikbaar waren (incubatie met strooisel) en dat strooisel sneller afbrak dan in het geval van de inheemse plantensoort. Mijn conclusie is daarom dat in specifieke gevallen areaaluitbreiders kunnen profiteren van plant-bodem terugkoppelingen via strooisel.

Er is een wisselwerking tussen effecten van areaaluitbreiding en directe effecten van klimaatverandering, zoals droogte, op het functioneren van de nieuwe leefgebieden. Ik onderzocht hoe areaaluitbreiders en bodemgemeenschappen uit het noorden en zuiden van Europa het functioneren van de bodem tijdens en na een periode van droogte beïnvloedden. Ik verwachtte dat plantengemeenschappen uit het zuiden beter waren aangepast aan droogte dan die uit het noorden en dus dat die bodems minder sterk zouden reagerem op droogte en sneller zouden herstellen, vooral in combinatie met bodemgemeenschappen uit het zuiden. In een mesocosmos experiment had ik plantengemeenschappen van inheemse en areaaluitbreidende soorten gecreëerd met en 
zonder bodemgemeenschappen uit het zuidelijke areaal. Ik heb bodemfuncties (strooiselafbraak, koolstofmineralisatie en de activiteit van enzymen), microbiële biomassa en de relatieve abundantie van saprofytische schimmels gemeten direct na de droogte en na een periode van 6 en 12 weken herstel. Het effect van droogte was hetzelfde voor alle planten- en bodemgemeenschappen, echter, tijdens de herstelperiode was strooiselafbraak sneller en de relatieve abundantie van sapropfytische schimmels hoger in bodems van inheemse planten die vlak daarvóór waren blootgesteld aan droogte, dan in bodems die geen droogtebehandeling hadden gehad. In tegenstelling daarmee werden bodemfuncties niet gestimuleerd in bodems van areaaluitbreidende planten met een historie van droogte. De herkomst van de bodemgemeenschap had weinig effect op de gemeten bodemfuncties. Na 12 weken waren alle bodemfuncties hersteld voor alle bodems en plantengemeenschappen. Deze resultaten laten zien dat wisselwerkingen tussen droogte en areaaluitbreiding vooral optreden tijdens het vroege herstel na een extreem-weer gebeurtenis.

Met dit onderzoek heb ik bijgedragen het begrijpen van plant-bodem interacties van areaaluitbreidende planten in hun nieuwe leefgebied. Ik heb laten zien dat de effecten van areaaluitbreidende soorten op de samenstelling en het functioneren van bodemgemeenschappen niet overeenkomen met effecten die we kennen voor intercontinentale invasieve plantensoorten. Uiteindelijk, als alle factoren gelijk worden gehouden, blijken areaaluitbreiders dezelfde effecten te hebben op de samenstelling en het functioneren van de bodemgemeenschap als inheemse plantensoorten uit hetzelfde geslacht. Desalniettemin, waren er specifieke gevallen waarin areaaluitbreiders meer profiteerden van hoge beschikbaarheid van voedingsstoffen en plantbodemterugkoppelingen via strooisel dan inheemse planten. Tot slot heb ik laten zien dat de grootste verschillen in plant-bodem interacties tussen areaaluitbreiders en inheemse planten mogelijk optreden tijdens het herstel na extreme weersomstandigheden, zoals na een droogteperiode. Om beter te begrijpen hoe areaaluitbreiders plant-bodem interacties beïnvloeden is het nodig dat veldstudies worden uitgevoerd waarin plant-bodem terugkoppelingen via strooisel worden onderzocht. Daarnaast is het nodig om experimenten te doen waarbij inheemse plantensoorten en areaaluitbreiders worden bestudeerd in competitie. Het is ook essentieel dat in vervolgstudies naar de effecten van klimaarveranderingen op het functioneren van ecosystemen rekening gehouden wordt met verschuivingen in de samenstellinging van de plantengemeenschappen door klimaatverandering. 


\section{Acknowledgments}

This PhD has been a special and memorable journey, an intense and enriching work and life experience for me. I am enormously grateful to all of you - colleagues, friends and family - who supported and helped me in many ways during the last years. I feel enormously rich with all I learned from you and lucky for the many good friends I made during this time.

Wim and Ciska, you have been the most supportive supervisors to me. Thank you for your confidence and constant guidance during this $\mathrm{PhD}$ journey. Wim, you always challenged me to think out of the box and to bring in new perspectives during our meetings. You gave me freedom to develop my own ideas and kept me motivated throughout the PhD. I admire your outstanding ability to make complicated things seem easy. I appreciated your openness and empathy during our conversations. Thank you for your compliments and for teaching me how to take them, even though it was difficult at times. Ciska, it is an honour to be your first PhD graduate. Thank you for all the discussions we had and for making time when I walked in your office with random questions, self-doubts or practical issues. You always gave constructive, clear and precise feedback on the dozens of drafts I sent you. Your comments and suggestions helped me structure the messy pile of ideas I had in my mind sometimes. You have a great attitude towards collaborative science and the future, and I really hope you will have the opportunity to continue to develop your own research ideas: now that decomposition has made it to TE, it better stays!

I would like to thank all the members of the ERC-team. Carolin, what would have I done without you? Thank you for your help, patience and ideas during the different experiments. Your work was always methodic and organized, and I appreciated your care for details. Janneke and Freddy, thank you for introducing me to the molecular lab, sharing all your tips and tricks and the fun times we had working together. Basten, I appreciate your enthusiasm and involvement in the rhizosphere chapter, your ability to handle sequencing data and to translate graph ideas into R codes. Olga, statistics were desperating at times, but I always felt so much better after talking to you. Stefan, thank you for all your advice and your relaxed and helpful attitude. Rutger, it has been a pleasure to share PhD journeys in this ERC, thank you for all your help and for organizing the awesome writing weeks. Kelly, thank you for welcoming me to your home in Duvall and for the good times we had in ESA Portland and in Wageningen. Thank you for your needed work supporting women scientists and advocating for equality in science. Kadri, you managed to find a positive side to nearly everything, science or life. Thank you for your contagious optimism and for your friendship. I wish Estonia was a little closer! In addition, I would like to thank our collaborators along the European transect, especially Branko and Tatjana. 
Gregor, your work is so important to keep the fytotron organized and make sure that all works smoothly for everybody. I appreciated your patience and understanding with my many samples, and the candies on Friday afternoons as well! Eke, thank you for your kindness, wisdom and willingness to help, always. You are awesome! Iris, Ciska R., Hans, Slavica and Agaat, thank you for your advice during my work in the different labs. To my student Rosanne, thank you for your willingness to learn and for your help. Jasper, thank you for all the advice on statistics and encouragement. Emilia, thank you for your help with the fungi sequencing data. Joost, I very much enjoyed the many discussions we had, thank you for your input and advice.

Sigrid, your determination during the last stretch of your $\mathrm{PhD}$ was equally admirable and motivating to me. You continue to be my PhD hero! I am proud to be your paranimph and to have you as mine. Thank you for taking me to your home place in Boyl. I hope we can share more adventures here and there, on bike or foot, and hopefully in some mountains soon.

Maddy \& Katja, you arrived to the department not that long ago but to me it seems we know each other for much longer. Thank you for your kindness and support during the last bit of the $\mathrm{PhD}$, and for initiating the Wednesday dinner tradition that certainly warmed up and spiced up our mid-week-deep. Thank you to the crowd - Stefan, Sigrid, Rutger, Tis, Robin, Heike, Casper, Jon - for the fun evenings.

Viola, Minghui, Cong, Haikun, Julio, Agata, Coti, Cunzheng, thank you for the good times we shared in the office. Sophie, I really appreciated your help with R.

I would like to thank all the people that have been part of the Terrestrial Ecology Department during my time here for the positive working atmosphere and the insightful discussions. I am also thankful to the fellow members of the VDPP organization and the Party Planners for the good and fun times shared. Thank you to the NIOO support staff Elly N, Gerrie, Ed, Gerda, Els, Dick - for making our lives a bit easier! In the NIOO, I enjoyed the great working atmosphere and facilities. But above all, I am really thankful for the great human value and authenticity I found in this Institute.

I would also like thank the people of the PE\&RC graduate school, and especially Claudius and Lennart, for helping us through during our $\mathrm{PhD}$ time.

Paolo, I feel lucky to be part of your Wageningen famiglia. Thank you for the many great moments we shared both at the NIOO and outside. I am proud to have you as my paranimph and of your determination to complete the $\mathrm{PhD}$, almost there! Antonella, I admire your work-hard and live-hard philosophy to life and I am thankful to have shared some bits of it. Kim \& Cam, thank you for all the nice time we had together, you are the sweetest Canadian duo and I promise to come to your next wedding. 
Annelies, it was great to get to meet you back in 2015. We shared countless bike trips and adventures. I love your spontaneity and sense of direction, which has taken us to many cool locations during our rides. Thank you for your support and advice. Julie, you always managed to make me feel calm after our conversations in the office and at home. Thank you for your serene perspective on everything. I loved visiting you and Pierre in Rennes. Ruth, thank you for all the good conversations we had, your care, kindness and empathy. Maaike, I really loved visiting your little boat house in the Thames. You are an inspiring traveler and I hope I get to share some outdoors with you sometime.

I would like to thank Sui, Thijs, Stijn, Sam, Nico, Nori, Kay, Sarash, Marika, Tania, Antica, Thomas L, Mandy, Sven, Laura \& Debora. You are beautiful and caring people and I am very glad our paths came together in Wageningen. Thank you for the many good conversations and fun we had, and for your support at different times and throughout this PhD journey.

Natalie, thank you for your support and encouragement at start of this PhD journey. I am thankful for the many good times we shared, and I wish you to find your happiness in life.

Judith, it was fun to share a flat with you during the first months of my PhD. Getting back to Wageningen turn out to be more difficult than expected, but I am thankful you helped me make my return a little easier.

Rima, it has been great fun to go on bike trips together. I hope we keep this annual tradition in the future somehow. Good luck with the rest of your PhD!

Charlotte, thank you for your patience and understanding during our conversations and for showing me the useful toolbox of emotional self-care.

Mirjam, we met in Wageningen 8 years ago (I know, time flies!). During this time, you have been my supervisor, teacher, colleague, wise advisor, inspiring adventurer and friend. Thank you for all!

Raimon \& Lucía, gracias por los ánimos y el buen humor. Ha sido un placer ver crecer a vuestra familia y os deseo lo mejor en vuestro nuevo hogar.

Nana y Rubén, mil gracias por esas reuniones anuales que dan la sensación que no estamos tan lejos. Qué bien lo hemos pasado y cuánto nos hemos reído! Espero que la tradición se mantenga y, sea donde sea, nos sigamos encontrando con mil y una historias que contar.

Vasco, my travel buddy. Thank you for the awesome time we had hiking and discovering new mountains in Norway and Nepal. Our trip to Nepal was a dream coming true for me, and I am so glad we went together. Thanks for all your wise advice during the PhD. I cannot wait to come visit you and Cassie soon. 
Colin, you are a warmhearted and caring men, and you helped me find my path. Thank you for taking me to the mountains of Skye and for your visit in Catalunya. I hope we get to share many more trails. Thank you (and Jasmina) for the beautiful cover.

Lucille, it would be very difficult to imagine the last few years without the house we shared and without you as a flat mate. We met randomly, just as part of the randomness of Wageningen, and quickly decided to get a place together, which turn out to be a great decision. There have been times of all colors since then, and I want to thank you for being there always. You have helped me bring balance to my life in times it was very difficult to find it, and I will always be grateful for that. I admire your resilience in many aspects of life, and your deep passion for little (non-human) creatures. Thanks to you I have also learned a lot about insects, gardening and proper French cuisine. Merci pour tout!

I evidentment no m'oblido de la gent de Gironella. De la Jasmina, la Judith, el Xevi, la Laura, el Pol, la Sílvia, el Guillem, la Nia, l'Arlet, l'Alba, el Noé, la Júlia, la Marina, la Sandra, el Xoy, la Lola, la Mariona, el Carles i la Martina. Gràcies per la vostra amistat tot i la distància. Em sento molt afortunada de poder tornar a casa i retrobar-vos sempre amb aquella il·lusió de qui fa temps que no es veu, però també amb aquella normalitat de qui $\mathrm{s}^{\prime}$ ha vist fa una setmana. És un plaer veure créixer les vostres famílies i els vostres projectes, i m'agradaria tenir-vos una mica més a prop. Mil gràcies per les visites que $\mathrm{m}^{\prime}$ heu anat fent durant aquests anys i que sens dubte $\mathrm{m}^{\prime}$ han fet sentir més a prop de casa. Jasmina, gràcies per transformar una conversa de cinc minuts en un disseny de portada. T'admiro. Xevi i Lola, els meus lingüistes, gràcies per revisar el resum de la tesi.

Gràcies també a la Maria, l'Adrià, l'Àngels, la Mariona i l'Arnau per l'acollida sempre que sóc a Gironella. M'agradaria passar més temps amb vosaltres també. Sou molt bona gent! Glòria, gràcies pel teu entusiasme. Anna, gràcies pels bons moments que hem compartit aquests anys i pels ànims.

Als pares, al Sergi, a les àvies, i als avis: Gràcies per tant! Gràcies per tenir tanta paciència amb mi i pel vostre suport incondicional. Sé que les meves decisions no sempre han estat fàcils per a vosaltres, però us agraeixo profundament el vostre esforç per entendre-les i la llibertat que sempre m'heu donat. Aquest doctorat també és part vostra. Vosaltres m’heu ajudat a ser qui sóc i us n'estaré sempre agraïda. 


\section{About the author}

Marta Manrubia Freixa was born on June $3^{\text {rd }}$ 1987. She grew up in Gironella, a small village in Catalonia, half-way between the Mediterranean Sea and the Pyrenees. At young age, Marta thought she would become a gardener and enjoyed spending time outdoors, especially in the forest and the mountains. In Gironella, Marta

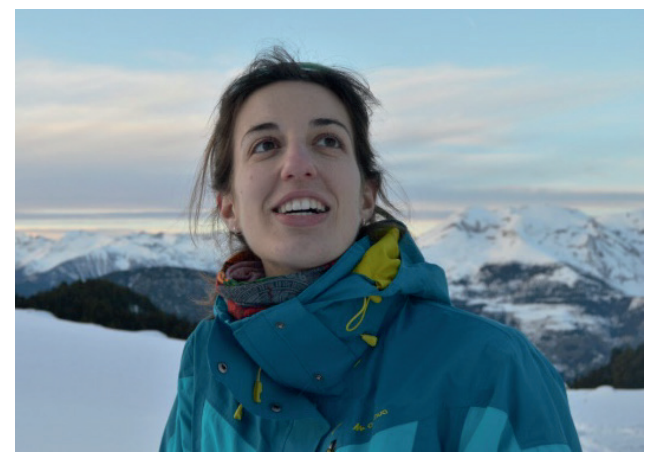
attended highschool in IES Pere Fontdevila. After, she studied a BSc in Biology at the University of Barcelona. During the last year of BSc, she did an internship at Wageningen University, in the Department of Soil Quality, to study how different agricultural management options influenced soil biological and physical parameters. She enjoyed living and studying in Wageningen and continued her studies in Wageningen University with an MSc in Earth \& Environment with a focus on Soil Ecology. During her MSc thesis, she studied how different mixtures of commercially sown grass species affected the emission of $\mathrm{N}_{2} \mathrm{O}$, a powerful greenhouse gas, from the soil. For her MSc internship project, Marta went to the James Hutton Institute in Dundee (Scotland). There, she learned multiple molecular techniques in soil ecology while investigating how root exudates steer denitrifying communities and $\mathrm{N}_{2} \mathrm{O}$ emissions from the soil. After her MSc graduation, Marta continued to work at the James Hutton Institute as a research assistant. In early 2014, Marta returned to Wageningen to start a PhD project on plant range-expansions and litter decomposition under the supervision of Wim van der Putten and Ciska Veen. During the last 4 years, Marta has been part of the Terrestrial Ecology Department of the Netherlands Institute of Ecology. Besides her work, Marta is an outdoor enthusiast and continues to enjoy her time in the mountains, participating in orienteering events and travelling on her bicycle. 


\section{List of publications}

Bradford, M.A., Veen, G.F., Bonis, A., Bradford, E.M., Classen, A.T., Cornelissen, J.H.C., Crowther, T.W., De Long, J.R., Freschet, G.T., Kardol, P., Manrubia-Freixa, M. Maynard, D.S., Newman, G.S., Logtestijn, R.S.P., Viketoft, M., Wardle, D.A., Wieder, W.R., Wood, S.A. \& van der Putten, W.H. (2017). A test of the hierarchical model of litter decomposition. Nature Ecology and Evolution,1, 1836-1845.doi:10.1038/s41559-017-0367-4

Langarica-Fuentes, A., Manrubia, M. Giles, M.E., Mitchell, S., \& Daniell, T.J. (2018). Effect of model root exudate on denitrifier community dynamics and activity at different water-filled pore space levels in a fertilised soil. Soil Biology E Biochemistry, 120, 70-79. doi:10.1016/j.soilbio.2018.01.034

Di Lonardo, P., Manrubia, M. De Boer, W., Zweers, A. J., Veen, G. F., \& Van der Wal, A. (2018). Relationship between home-field advantage of litter decomposition and priming of soil organic matter. Soil Biology \& Biochemistry, 126, 4956. doi:10.1016/j.soilbio.2018.07.025 


\section{PE\&RC Training and Education Statement}

With the training and education activities listed below the PhD candidate has complied with the requirements set by the C.T. de Wit Graduate School for Production Ecology and Resource Conservation (PE\&RC) which comprises of a minimum total of 32 ECTS (= 22 weeks of activities)

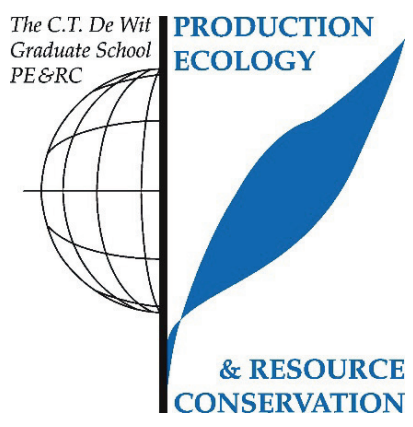

\section{Review of literature (5 ECTS)}

- Climate change, plant range shifts, and decomposition of carbon inputs in the soil

\section{Writing of project proposal (4.5 ECTS)}

- $\quad$ Litter decomposition and Home-Field Advantage during plant range shifts

\section{Post-graduate courses (7.1 ECTS)}

- Introduction to R for statistical analyses; PE\&RC (2014)

- Soil ecology and the planetary boundaries; PE\&RC (2016)

- Multivariate analyses of ecological data using CANOCO; University of South Bohemia, Czech Republic (2017)

Invited review of (unpublished) journal manuscript (2 ECTS)

- Plant \& Soil: climate change effects on plant litter and decomposition (2016)

- Plant \& Soil: plant soil feedbacks along a rainfall gradient (2017)

\section{Competence strengthening / skills courses (2.2 ECTS)}

- Competence assessment; WGS (2014)

- Reviewing a scientific paper; WGS (2018)

- Career perspectives; WGS (2018)

PE\&RC Annual meetings, seminars and the PE\&RC weekend (2.7 ECTS)

- PE\&RC Weekend - first year

- PE\&RC Weekend - mid

- PE\&RC Weekend - last year

- PE\&RC Day

- WPC 2nd Symposium 
Discussion groups / local seminars / other scientific meetings (5.8 ECTS)

- Ecological theory and application (2014-2016)

- $\quad$ NIOO Terrestrial Ecology monthly discussion group (2014-2017)

- $\quad$ NIOO Science days $(2015,2017)$

International symposia, workshops and conferences (15.5 ECTS)

- C Cycling: from plants to ecosystems; poster presentation; Manchester (2014)

- GSBI; poster presentation; Dijon (2014)

- Rhizo4; poster presentation; Maastricht (2015)

- ESA; oral presentation; Portland (2017)

- Ecology across borders: BES joint meeting; poster presentation; Ghent (2017)

- NAEM; poster presentation $(2015,2016)$; oral presentation (2017)

Lecturing / Supervision of practicals / tutorials (3 ECTS)

- Supervision of MBO student (2016) 


\section{Colophon}

The research described in this thesis was conducted at the Department of Terrestrial Ecology of the Netherlands Institute of Ecology (NIOO-KNAW) and financially supported by the European Research Council (ERC) as part of an ERC Advanced Grant to W.H.v.d.P. (ERC-Adv 26055290 SPECIALS).

This is NIOO thesis 161

Text, figures and layout: Marta Manrubia Freixa

Summary in Dutch: Ciska Veen

Pictures: Freddy ten Hooven (p.8, p.108), Marta Manrubia (p.22, p.44, p.76, p.136), Mariona Casas (p. 178)

Cover and cover design: Jasmina Serri \& Colin Sinclair

Printed by: Digiforce I Proefschriftmaken.nl 




\section{Propositions}

1. In novel environments, effects of range-expanding plants and related natives on rhizosphere bacteria are indistinguishable.

(this thesis)

2. Understanding the responses of terrestrial ecosystems to climate change requires testing effects of plant community changes in multifactorial experiments. (this thesis)

3. A main challenge in ecological studies is finding the balance between mechanistic understanding and relevance in the field.

4. Given that failure is an integral part of the scientific method, negative and positive results are equally valuable.

5. Valuing publication of peer-reviewed studies in scientific journals over other communication channels hampers bridging between science and society.

6. Mental hygiene is as important as brushing your teeth.

Propositions belonging to the thesis entitled:

"Plant-soil interactions of range-expanding plants"

Marta Manrubia Freixa

Wageningen, 7 December 2018 